Florida International University

FIU Digital Commons

FIU Electronic Theses and Dissertations

University Graduate School

$11-7-2014$

\title{
Development of Hardware in the Loop Real-Time Control Techniques for Hybrid Power Systems Involving Distributed Demands and Sustainable Energy Sources
}

Ali Mazloomzadeh

amazl001@fiu.edu

DOI: $10.25148 /$ etd.FI14110754

Follow this and additional works at: https:// digitalcommons.fiu.edu/etd

Part of the Electrical and Electronics Commons, Engineering Education Commons, Other Electrical and Computer Engineering Commons, and the Power and Energy Commons

\section{Recommended Citation}

Mazloomzadeh, Ali, "Development of Hardware in the Loop Real-Time Control Techniques for Hybrid Power Systems Involving Distributed Demands and Sustainable Energy Sources" (2014). FIU Electronic Theses and Dissertations. 1666.

https://digitalcommons.fiu.edu/etd/1666 


\title{
FLORIDA INTERNATIONAL UNIVERSITY \\ Miami, Florida
}

\section{DEVELOPMENT OF HARDWARE IN THE LOOP REAL-TIME CONTROL TECHNIQUES FOR HYBRID POWER SYSTEMS INVOLVING DISTRIBUTED DEMANDS AND SUSTAINABLE ENERGY SOURCES}

\author{
A dissertation submitted in partial fulfillment of \\ the requirements for the degree of \\ DOCTOR OF PHILOSOPHY \\ in \\ ELECTRICAL ENGINEERING \\ by
}

Ali Mazloomzadeh 
To: Dean Amir Mirmiran

College of Engineering and Computing

This dissertation, written by Ali Mazloomzadeh, and entitled Development of Hardware in the Loop Real-Time Control Techniques for Hybrid Power Systems Involving Distributed Demands and Sustainable Energy Sources, having been approved in respect to style and intellectual content, is referred to you for judgment.

We have read this dissertation and recommend that it be approved.

$\begin{array}{r}\text { Mark Roberts } \\ \hline \text { Hector R. Fuentes } \\ \hline \text { Nezih Pala } \\ \hline \text { Osama A. Mohammed, Major Professor }\end{array}$

Date of Defense: November 7, 2014

The dissertation of Ali Mazloomzadeh is approved.

Dean Amir Mirmiran

College of Engineering and Computing

Dean Lakshmi N. Reddi

University Graduate School

Florida International University, 2014 
(C) Copyright 2014 by Ali Mazloomzadeh

All rights reserved. 


\section{DEDICATION}

I dedicate this dissertation to my dear wife, Noushin Mesriyan, whose patience, sacrifice, support, and understanding made the completion of this work possible, and to my beloved family who were unflinching in their support and prayers even from thousands of miles away. 


\section{ACKNOWLEDGMENTS}

I would like to give my sincere appreciation and gratitude to my major Professor

Osama A. Mohammed for his support, presence, guidance, and expert contribution though the period of research and dissertation development, without which it was would not been possible to accomplish. I also appreciate Professor Mohammed's suggestions, advice, efforts and corrections during the development of this dissertation. I was honored to serve as teaching and research assistant, and be the recipient of the best student paper award and university graduate school Dissertation Year Fellowship, by his continuous support and supervision.

I wish to also thank the members of my committee for their support, patience and valuable discussions, in particular, Dr. Nezih Pala, Dr. Mark Roberts, Dr. Hector R. Fuentes, and Dr. Stavros Georgakopoulos.

I would like to acknowledge the research support provided through the projects funded by the Office of Naval Research and the US Department of Energy throughout my years of dissertation research. I also acknowledge the dissertation year fellowship from the FIU graduate school during the last year of dissertation research.

I also like to appreciate my colleagues at the Department of Electrical and Computer Engineering at Florida International University, and the Energy Systems Research Laboratory graduate members specially Mr. Mehmet Hazar Cintuglu, and undergraduate student scholars, who assisted the completion of test setups during my Ph.D. research. I am also grateful to the FIU community, all my colleagues and department staff specially Mr. Oscar Silveira, who have given me an unforgettable memory, full of kindness and professionalism. 


\section{ABSTRACT OF THE DISSERTATION \\ DEVELOPMENT OF HARDWARE IN THE LOOP REAL-TIME CONTROL \\ TECHNIQUES FOR HYBRID POWER SYSTEMS INVOLVING DISTRIBUTED \\ DEMANDS AND SUSTAINABLE ENERGY SOURCES}

by

Ali Mazloomzadeh

Florida International University, 2014

Miami, Florida

\section{Professor Osama A. Mohammed, Major Professor}

The future power grid will effectively utilize renewable energy resources and distributed generation to respond to energy demand while incorporating information technology and communication infrastructure for their optimum operation. This dissertation contributes to the development of real-time techniques, for wide-area monitoring and secure real-time control and operation of hybrid power systems.

To handle the increased level of real-time data exchange, this dissertation develops a supervisory control and data acquisition (SCADA) system that is equipped with a state estimation scheme from the real-time data. This system is verified on a specially developed laboratory-based test bed facility, as a hardware and software platform, to emulate the actual scenarios of a real hybrid power system with the highest level of similarities and capabilities to practical utility systems. It includes phasor measurements at hundreds of measurement points on the system. These measurements were obtained from especially developed laboratory based Phasor Measurement Unit (PMU) that is utilized in addition to existing commercially based PMU's. The developed 
PMU was used in conjunction with the interconnected system along with the commercial PMU's. The tested studies included a new technique for detecting the partially islanded micro grids in addition to several real-time techniques for synchronization and parameter identifications of hybrid systems.

Moreover, due to numerous integration of renewable energy resources through DC microgrids, this dissertation performs several practical cases for improvement of interoperability of such systems. Moreover, increased number of small and dispersed generating stations and their need to connect fast and properly into the AC grids, urged this work to explore the challenges that arise in synchronization of generators to the grid and through introduction of a Dynamic Brake system to improve the process of connecting distributed generators to the power grid.

Real time operation and control requires data communication security. A research effort in this dissertation was developed based on Trusted Sensing Base (TSB) process for data communication security. The innovative TSB approach improves the security aspect of the power grid as a cyber-physical system. It is based on available GPS synchronization technology and provides protection against confidentiality attacks in critical power system infrastructures. 


\section{TABLE OF CONTENTS}

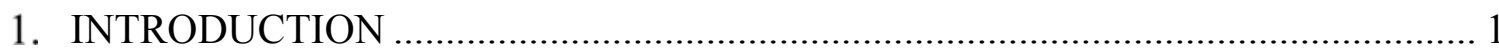

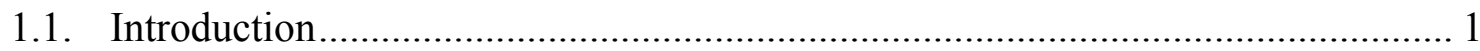

1.2. Current State of Power Grids .................................................................... 5

1.2.1. Smart/advanced metering infrastructure .....................................................5

1.2.2. Wide Area Monitoring, Protection and Control (WAMPAC) Systems ............6

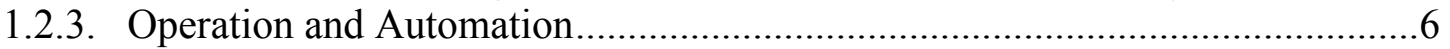

1.2.4. Information Technology and Communication Improvements.......................... 7

1.3. Motivation for Solving the Existing Problems ......................................... 7

1.3.1. Essential applications are in practice .....................................................

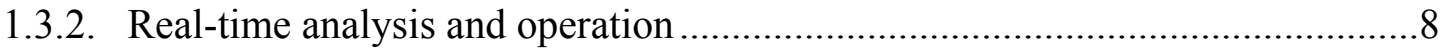

1.3.3. Verifications of developed techniques on hardware based testbed ..................9

1.3.4. Simulation systems with physical conditions, cyber security and

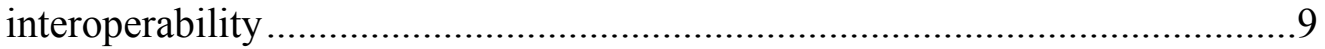

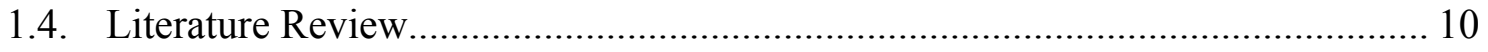

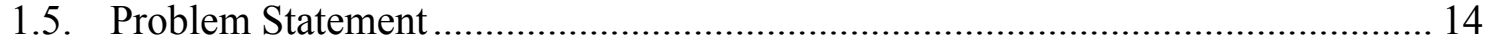

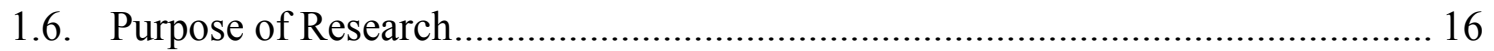

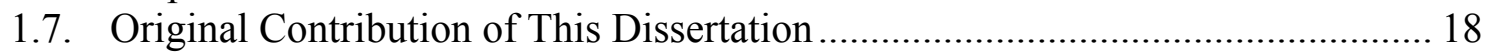

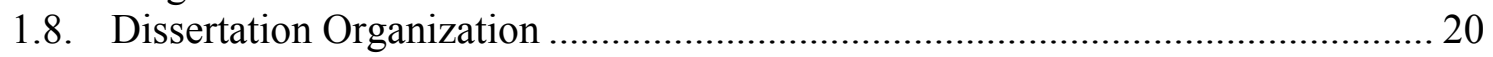

2. REAL-TIME OPERATIONAL ISSUES OF POWER SYSTEMS AND REQUIREMENTS TO ACHIEVE SECURE OPERATION ................................. 25

2.1. Future Power Systems Need Higher Security ................................................. 25

2.2. Future Power Systems are Hybrid and Involve Renewable Energy Resources.... 27

2.3. Future Power Grids Require Higher Level of Information Technology and Wide Area Communication Systems ….......................................................... 28

2.4. Synchrophasor Technology is Broadly Spread in Future Power Systems ............ 30

2.5. Requirements to Achieve secure and Successful Operation.............................. 39

3. DEVELOPMENT OF SCADA SYSTEM FOR REAL-TIME OPERATION OF POWER SYSTEMS ................................................................................ 41

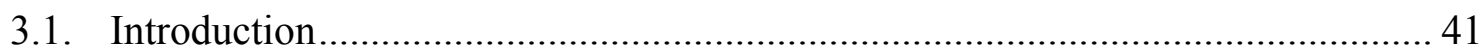

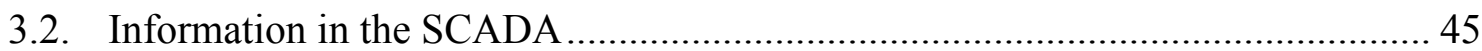

3.3. Requirements for Integration of a SCADA with Real-Time Capability.............. 45

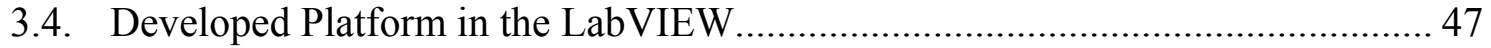

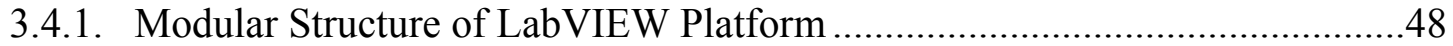

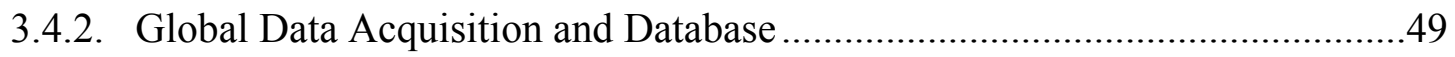

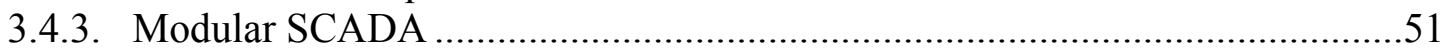

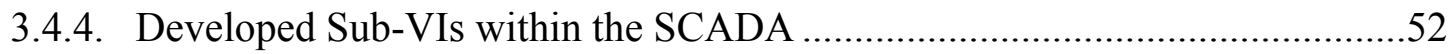

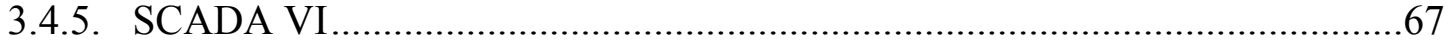


3.4.6. System Connectivity Online Monitoring VI...................................................71

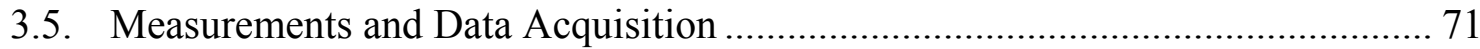

3.6. Calibration and Linearization of Measurements System ..................................... 73

\section{IMPROVEMENT OF MEASUREMENT SYSTEM THROUGH STATE}

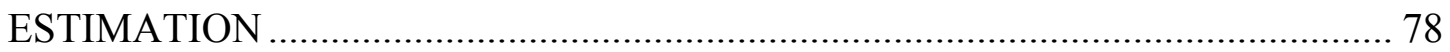

4.1.1. Precise Measurements and the Benefits for SCADA ………………….........79

4.1.2. Classical State Estimation Procedure Overview..............................................8

4.1.3. Hybrid State Estimation ...............................................................................85

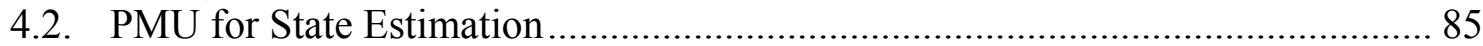

4.3. Suggested Methodology to Reduce the Estimation Error ..................................... 87

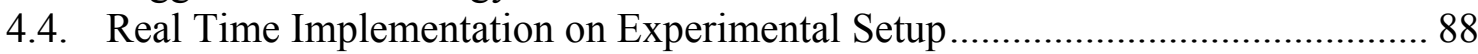

4.5. Integration of Real-Time Data Acquisition for State Estimation .......................... 88

4.5.1. State Estimation algorithm developed in Matlab ............................................89

4.5.2. Integration of Real-Time State Estimation (in LabVIEW Environment)........92

4.5.3. Performing State Estimation in the Commercial Software (DIgSILENT

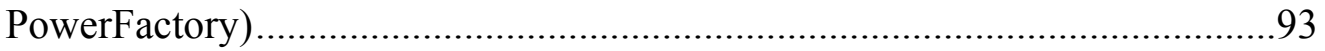

4.6. Post Processing: Detection and Elimination of Bad Data.................................... 95

4.7. Conclusion .................................................................................................... 97

5. TRUSTED SENSING BASE FOR SECURITY OF POWER GRID ……………..... 98

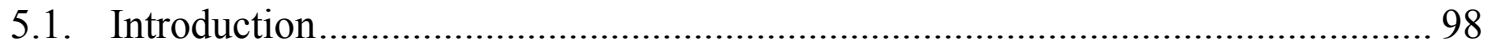

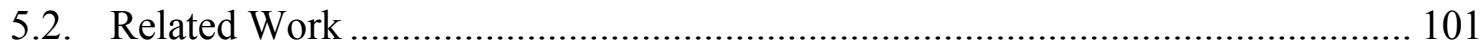

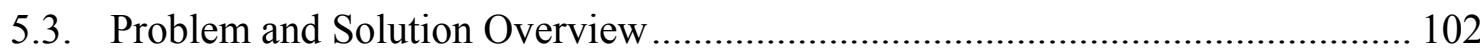

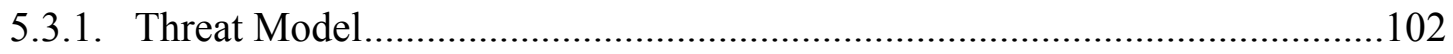

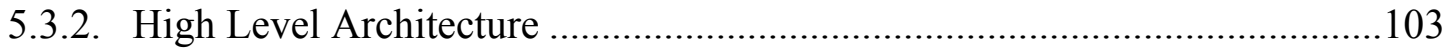

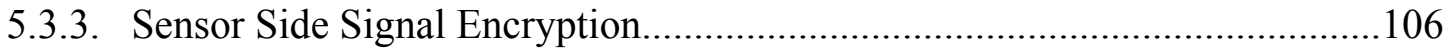

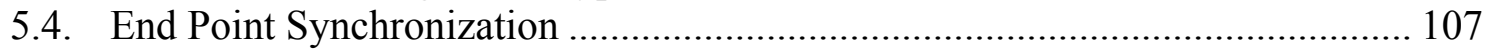

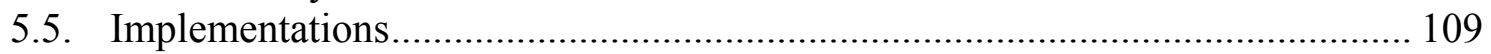

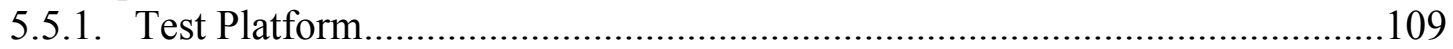

5.5.2. Hardware specifications .........................................................................109

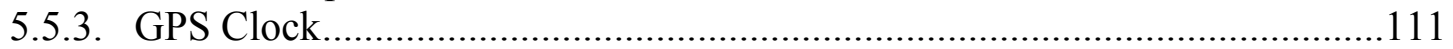

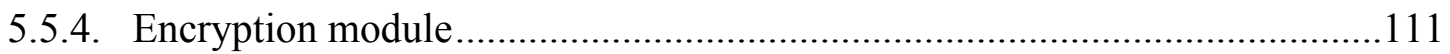

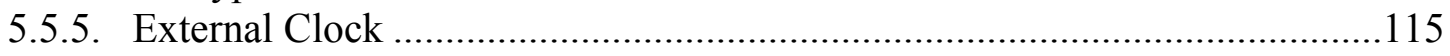

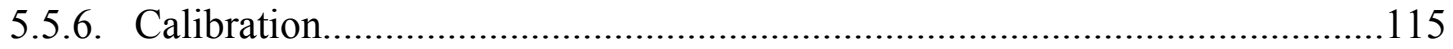

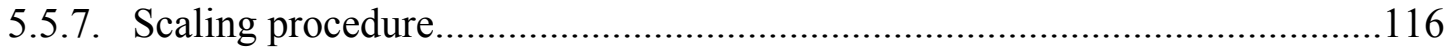

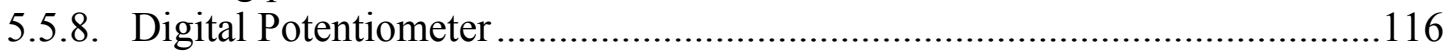

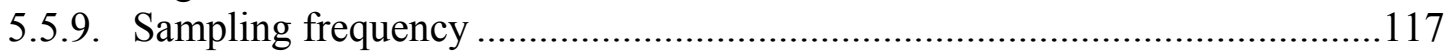

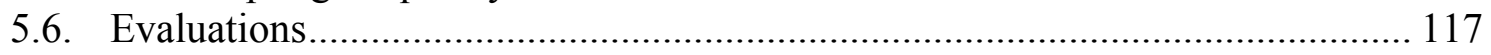

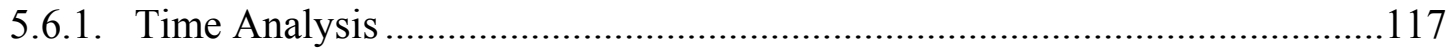

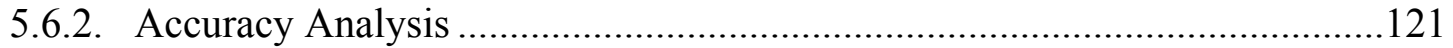

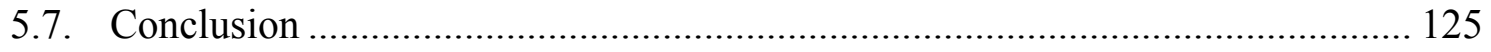




\section{DEVELOPMENT OF HARDWARE IN THE LOOP TECHNIQUES FOR}

MICROGRID CONNECTIVITY

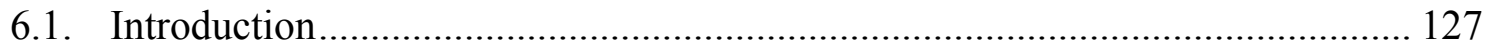

6.2. Benefits of DC Micro Grid Deployment ....................................................... 127

6.3. Integration of the Hybrid Grid by Development of AC/DC Microgrids ............ 129

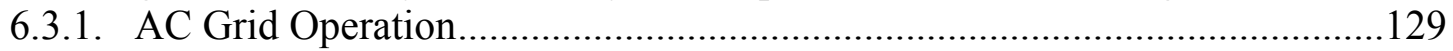

6.3.2. Hybrid Operation of Photo Voltaic Micro Grid for Sharing Power with

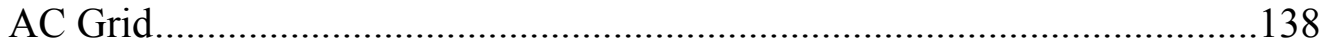

6.3.3. Frequency Regulation of Islanded Microgrid ...............................................140

7. THE REAL-TIME SMART GRID TESTBED …………………….................. 142

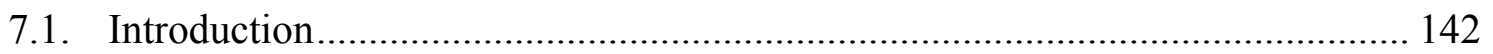

7.2. Generation Station Model ............................................................................ 145

7.3. Transmission Line Model ............................................................................. 152

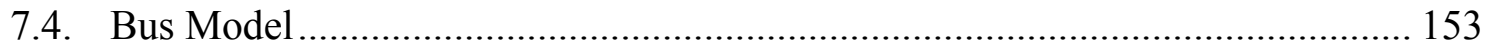

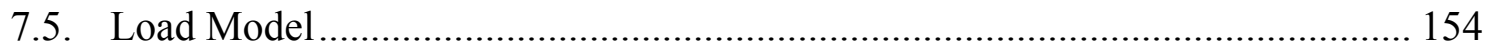

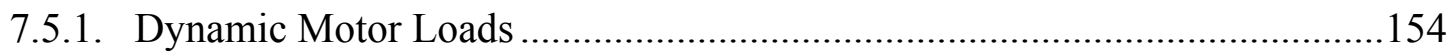

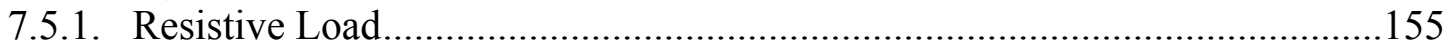

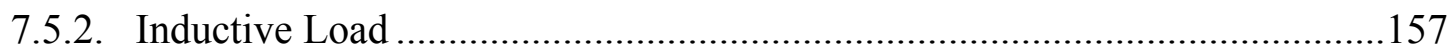

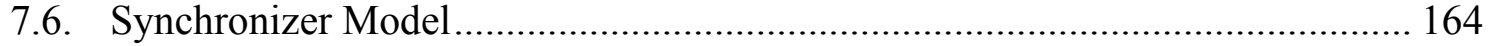

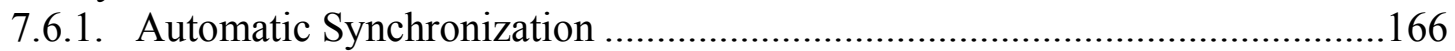

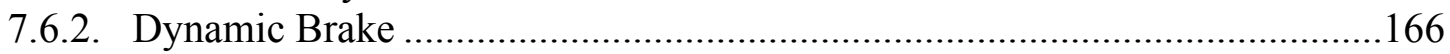

8. DEVELOPMENT OF HYBRID AC-DC POWER SYSTEMS INVOLVING

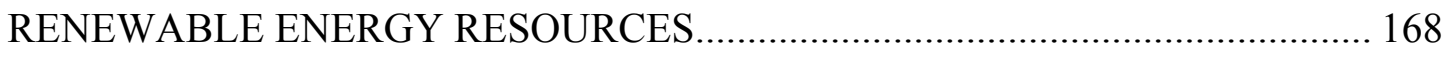

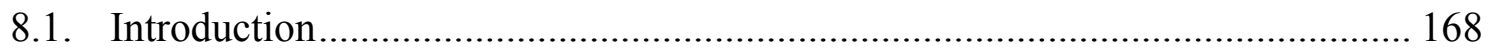

8.2. Implementation of Renewable Energy Resources .............................................. 169

8.3. Integrated Hybrid Power System .................................................................. 170

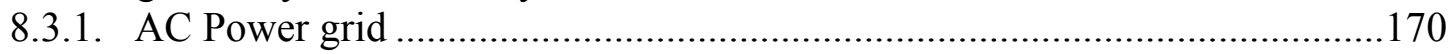

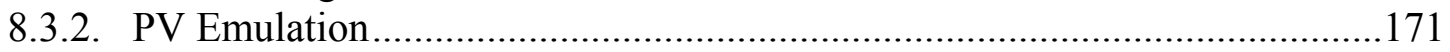

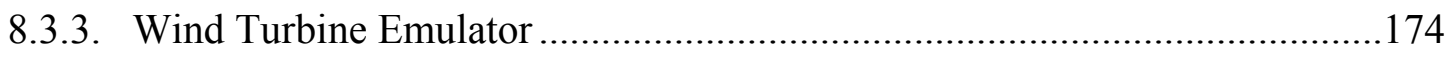

8.3.4. Energy Storage System ...........................................................................178

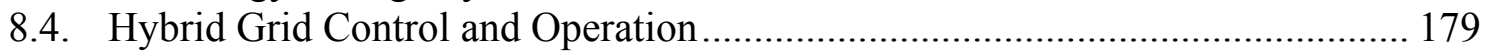

8.4.1. Isolated Microgrid Architecture and Operation .............................................179

8.4.2. Developing Procedures for Power Balancing in DC Grid ..............................180

8.4.3. Bidirectional Energy Conversion in DC Microgrid.......................................181

8.4.4. Bidirectional power flow in the interconnected $\mathrm{AC} / \mathrm{DC}$ grid for controlling the active and reactive power flow ...............................................184

8.5. Educational Aspects of the Developed Platform .............................................. 187

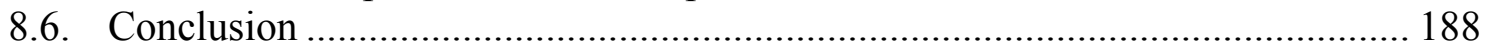

9. PHASOR MEASUREMENT UNITS IN SMART GRID ....................................... 189

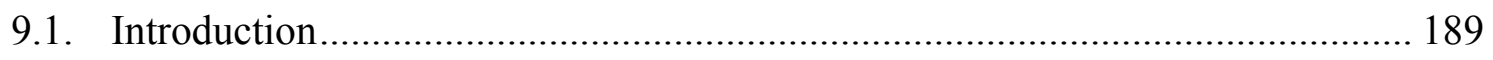




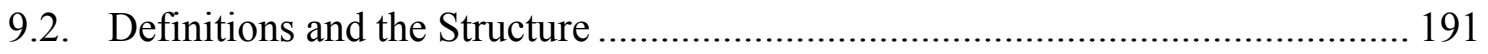

9.2.1. Synchronized Measurement in Power Systems ..............................................191

9.2.2. Global Time Reference and PMU basics..................................................191

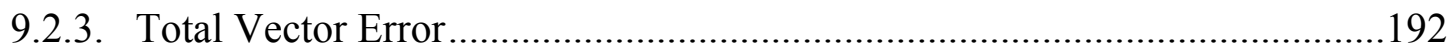

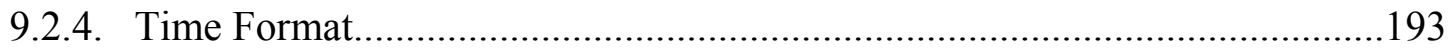

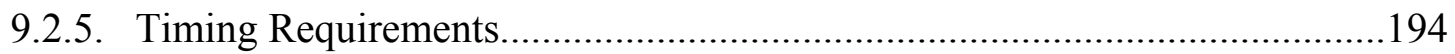

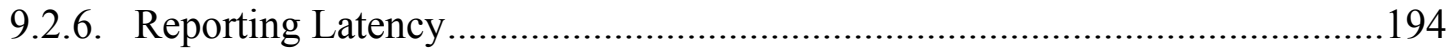

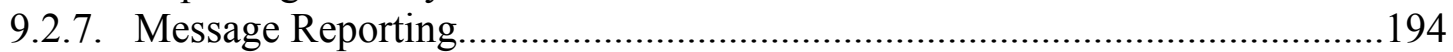

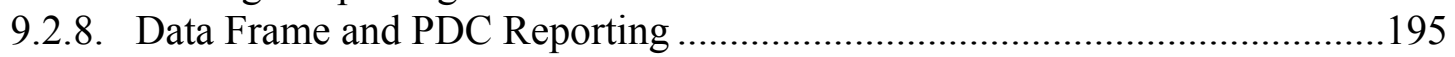

9.3. Development of Laboratory-Based Phasor Measurement Unit ............................ 196

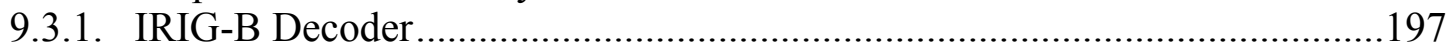

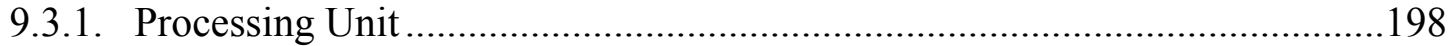

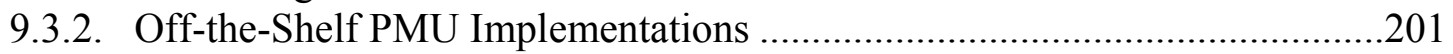

9.4. Validation, Calibration, and Performance Comparison ..................................... 202

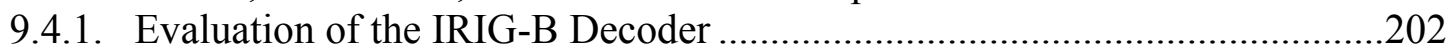

9.4.1. TVE Comparison between the Developed PMU and Commercial PMUs ....203

9.4.2. Evaluation of the captured Phase and Frequency ……………………..........208

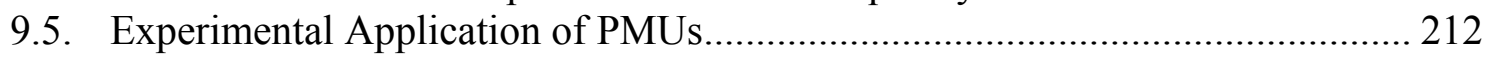

9.5.1. Transmission Line Parameter Identification ................................................22

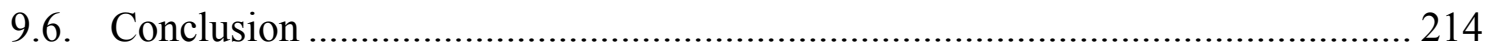

\section{DEVELOPMENT OF TECHNIQUES FOR ISLANDING DETECTION USING}

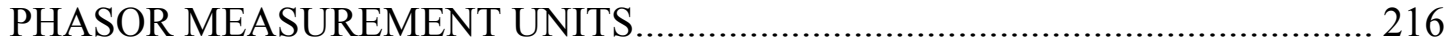

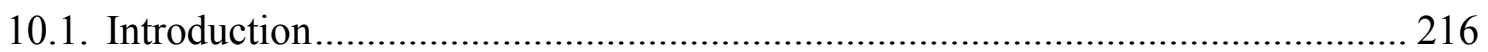

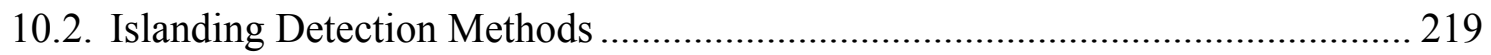

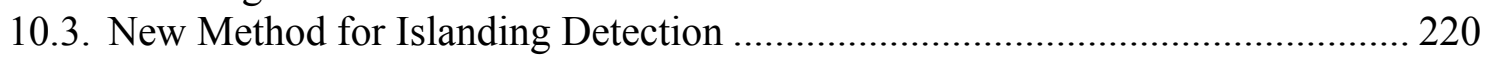

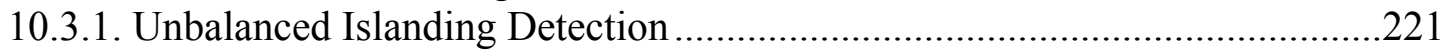

10.3.2. Inter-microgrid Synchronized Measurements................................................221

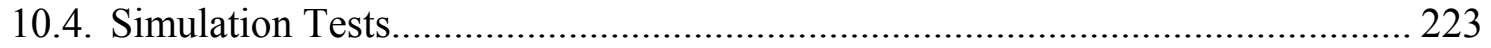

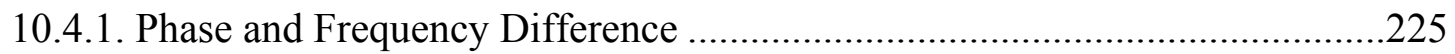

10.4.2. Unbalanced Islanding of Microgrid ............................................................22

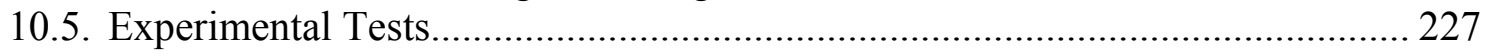

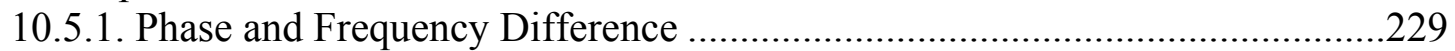

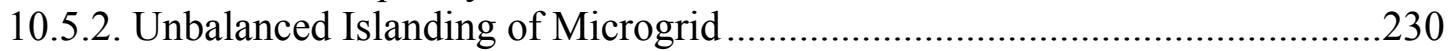

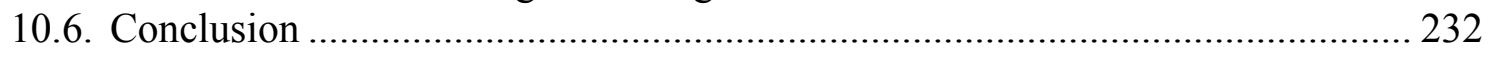

\section{DEVELOPMENT OF A SOFT SYNCHRONIZATION TECHNIQUE FOR} DISTRIBUTED GENERATING STATIONS ……………………………........ 234

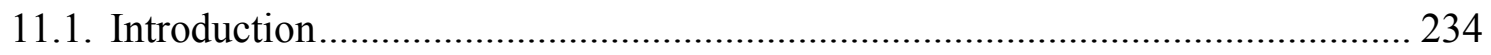

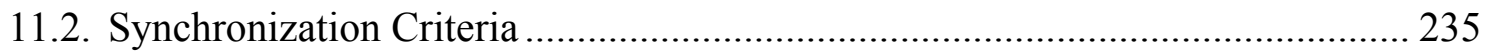

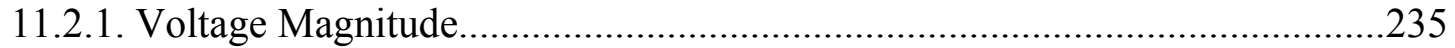

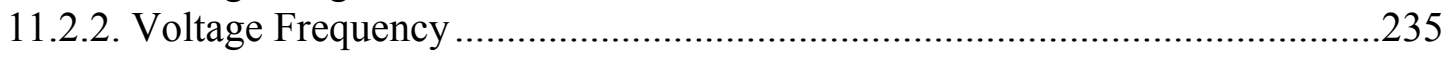

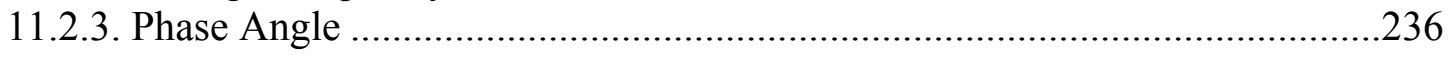

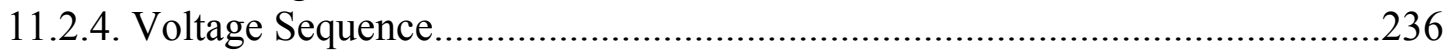




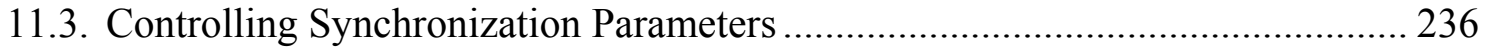

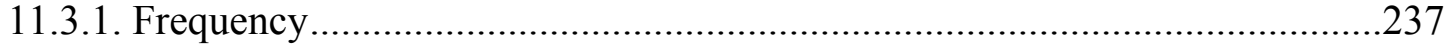

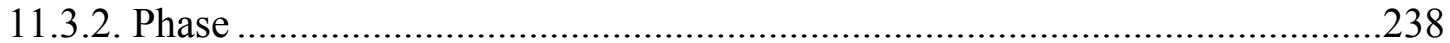

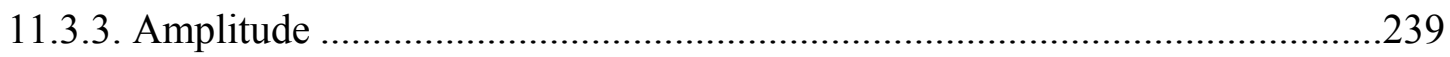

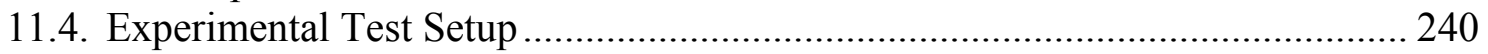

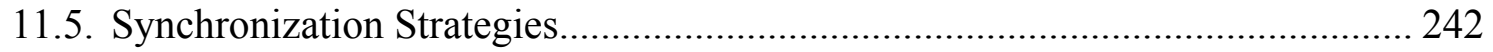

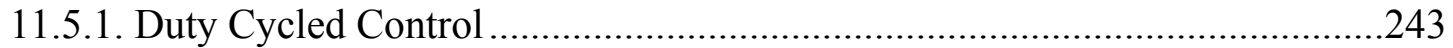

11.5.2. Duty Cycled Control with Constant Local Load ..........................................245

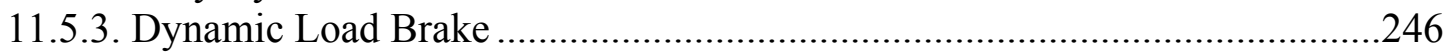

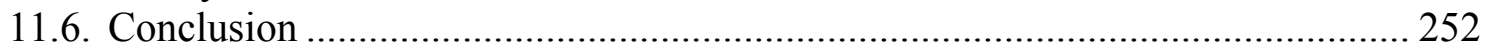

12. CONCLUSION AND RECOMMENDATIONS FOR FUTURE WORK.............. 253

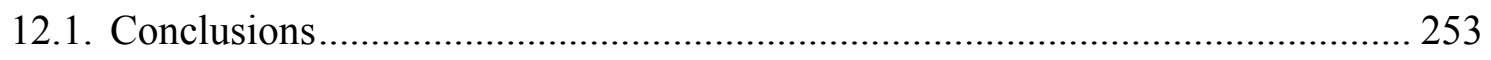

12.2. Recommendations for Future Works ....................................................... 257

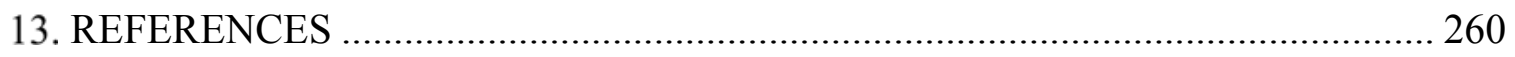




\section{LIST OF TABLES}

TABLE

PAGE

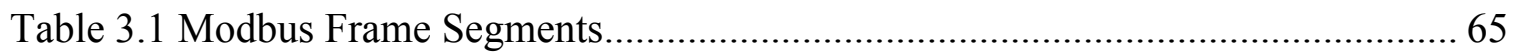

Table 4.1 Transmission Lines Parameters and Their Connectivity ................................. 88

Table 6.1 Interconnection of AC/DC System in Power Sharing ............................... 140

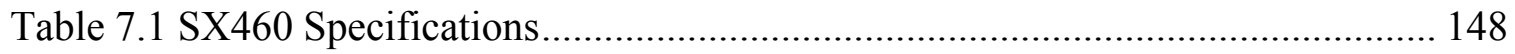

Table 7.2 Parameters of Synchronous Generators ................................................... 151

Table 7.3 Specifications of Developed Resistive Loads............................................ 155

Table 7.4 Specification of Inductive Loads ............................................................ 159

Table 9.1 Random results for phase and frequency measurements ........................... 211

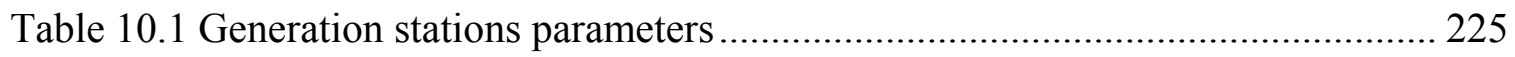

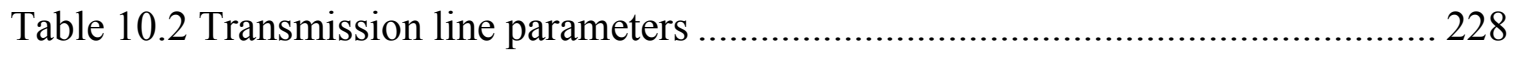

Table 11.1 Average synchronization time for each generation station for three discussed methods of controlling the frequency and phase....................... 250 


\section{LIST OF FIGURES}

FIGURE

PAGE

Figure 1.1 Traditional Power Grid structure ......................................................... 2

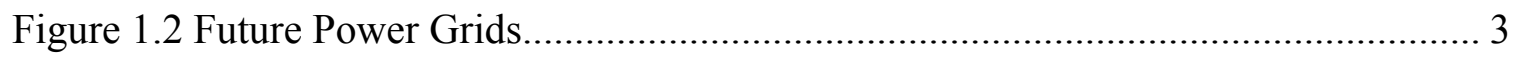

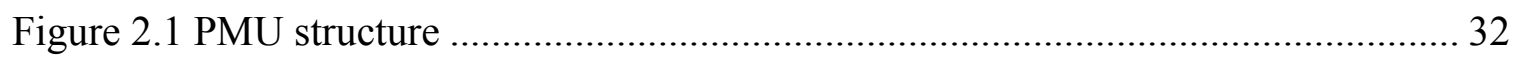

Figure 3.1 LabVIEW structure ........................................................................ 48

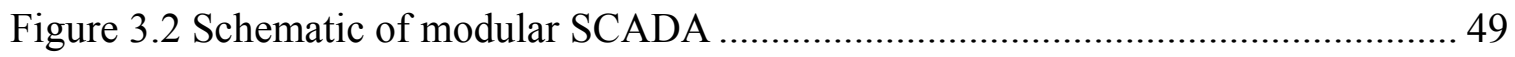

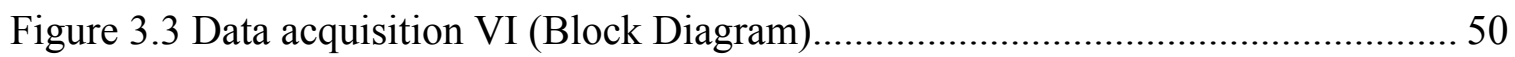

Figure 3.4 An example of modules used in the new SCADA ...................................... 51

Figure 3.5 Block diagram of: a) modular SCADA b) non-modular SCADA ................. 52

Figure 3.6 Simple block Diagram of measurement, calculation, and presentation of

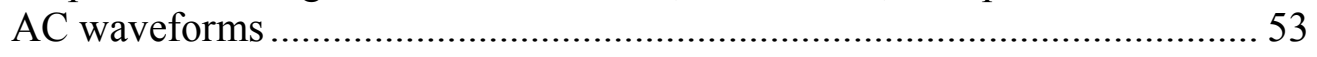

Figure 3.7 Schematics of synchronized measurement sub-VI.................................... 54

Figure 3.8 Synchronized measurement sub-block .................................................. 55

Figure 3.9 Block diagram of 3-phase measurement sub-VI ..................................... 55

Figure 3.10 Front panel of the preliminary 3-phase measurement VI.......................... 56

Figure 3.11 Front panel of the latest 3-phase measurement VI .................................. 56

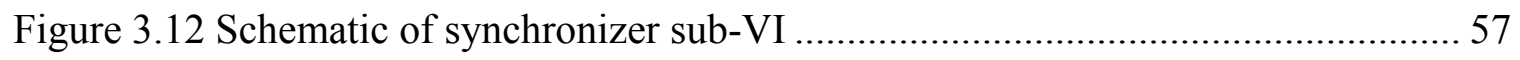

Figure 3.13 Block diagram of synchronizer sub-VI .............................................. 58

Figure 3.14 Front panel of synchronizer (old version) ............................................ 58

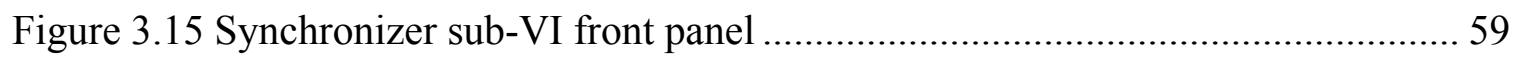

Figure 3.16 Front panel and block diagram of vector drawing sub-VI. ........................ 59

Figure 3.17 V-A demux sub-VI (a) block diagram (b) front panel ............................... 60

Figure 3.18 Watt-VAR demux sub-VI (a) block diagram (b) front panel ...................... 60

Figure 3.19 Phasor demux sub-VI (a) block diagram (b) front panel........................... 61 


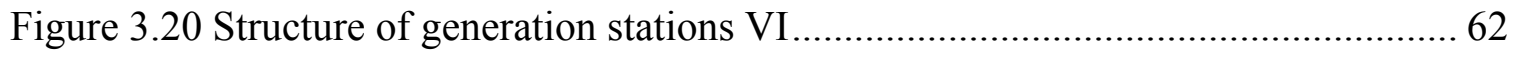

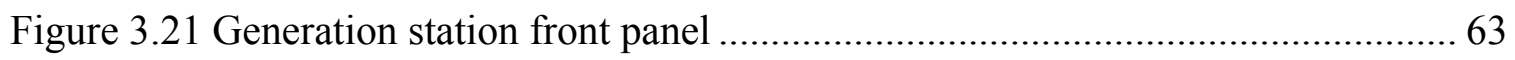

Figure 3.22 Comparison of active power generation in each station................................ 64

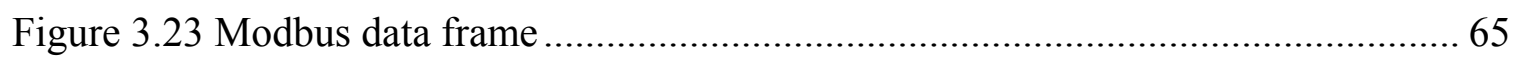

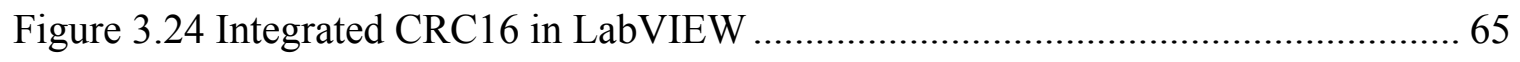

Figure 3.25 Block diagram of generation station control VI .......................................... 66

Figure 3.26 Developed stand-alone serial communication software ................................. 67

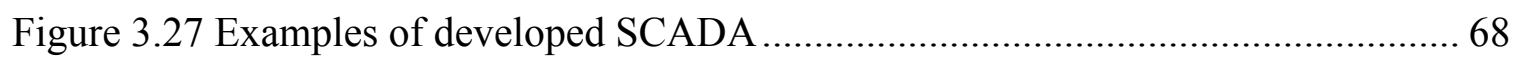

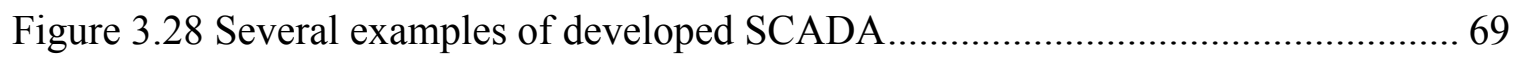

Figure 3.29 Comprehensive front panel of SCADA.................................................... 70

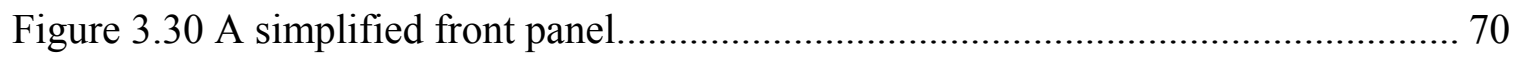

Figure 3.31 Online monitoring/presentation of SCADA …………………………....... 71

Figure 3.32 DAQs and measurement in testbed ...................................................... 72

Figure 3.33 Information flow through analog and digital signals in a power system ...... 73

Figure 3.34 Developed PT calibration module ………………………........................ 74

Figure 3.35 Developed CT calibration module.............................................................. 75

Figure 3.36 Interpolation of linearization inverse function ........................................... 75

Figure 3.37 Applying different orders of polynomial functions for linearization ............ 76

Figure 4.1 A Complete state estimation process............................................................. 82

Figure 4.2 Local isolated/deviated synchronized measurements in global power

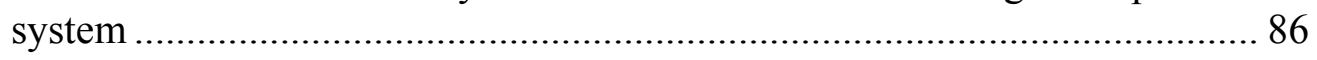

Figure 4.3 Separation of synchronized measurement (grouped by color) ......................... 86

Figure 4.4 PDC interface in LabVIEW for SEL PMUs and protection relays (courtesy of ESRL) ……......................................................................... 89

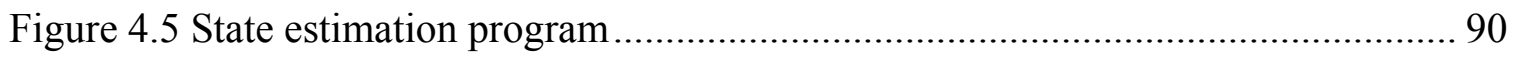


Figure 4.6 Exporting the SCADA information to state estimation interface. 92

Figure 4.7 Sequential block diagram of state estimation interface ............................... 93

Figure 4.8 Front panel of online analysis interface ............................................... 94

Figure 4.9 Load flow results (Left) compared to state estimation results (Right) ........... 94

Figure 4.10 An example of pre-processing in state estimation................................. 96

Figure 4.11 An example of post-processing and bad data detection. ............................ 96

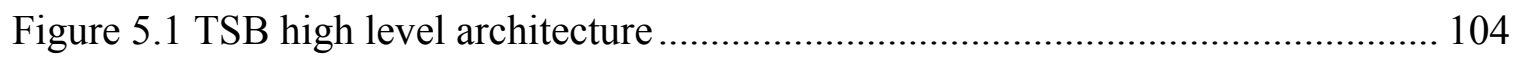

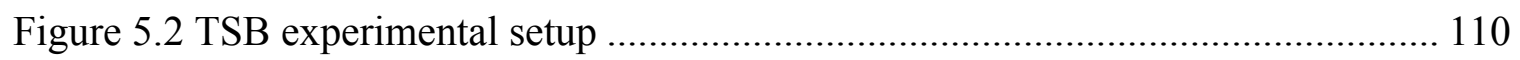

Figure 5.3 TSB environment and flow of signals .............................................. 112

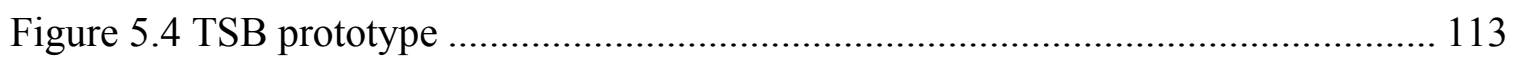

Figure 5.5 Association of communication channels with main process unit................. 114

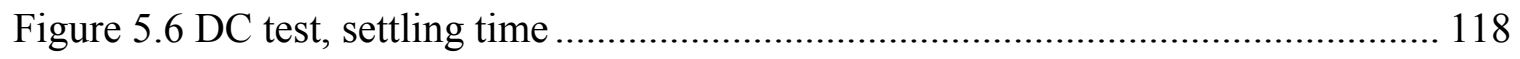

Figure 5.7 AC test, communication delay for RS232 command ............................... 119

Figure 5.8 AC test, communication delay for RS232 command ............................... 119

Figure 5.9 Autonomous scaling of signal triggered by IRIG-B.............................. 120

Figure 5.10 Total delay in autonomous operation triggered by IRIG-B...................... 121

Figure $5.110 \%$ Scaled signal in comparison to original signal................................ 122

Figure $5.12100 \%$ Scaled signal in comparison to original signal and showing their

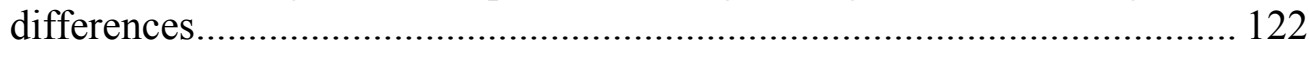

Figure 5.13128 Scales, expected, measured and \% error values .............................. 123

Figure 5.14 Comparison of original, encrypted and reconstructed waveforms ............. 124

Figure 5.15 PMU SEL-421 readings: (a) Original RMS readings (b) Original RMS readings (c)Encrypted RMS readings (d) Applied and actual scales (e) Reconstructed RMS values.

Figure 6.1 Step by step process of synchronization, 1) before, 2) at the beginning of the synchronization of $\mathrm{G} 2$ to bus0380 through synchronizer0420 ............. 131 
Figure 6.2 Step by step process of synchronization, 3) during, and 4) after synchronization of G2 to bus0380 through synchronizer0420.

Figure 6.3 Integration of dynamic brake, 1) before, 2) at the beginning of the synchronization process of G2 to bus0380 through synchronizer0420 ....... 132

Figure 6.4 Integration of dynamic brake, 3) during, and 4) after synchronization process of $\mathrm{G} 2$ to bus 0380 through synchronizer0420

Figure 6.5 System configuration in reactive load response study and corresponding inductive load controllers

Figure 6.6 Respond of G1/G2 on reactive power change at IL1 (Period of $1 \mathrm{~min}$.) (System of Figure 5.3)

Figure 6.7 Response of G1 and G2 to change of L1 and L2 (Resistive loads) (Presented in Figure 5.3).

Figure 6.8 The SCADA representation of the system for inductive and resistive load change.

Figure 6.9 Inductive and resistive load changes and their impact on the generator ....... 136

Figure 6.10 The SCADA representation of the system with PV and slack bus.............. 137

Figure 6.11 Power sharing between AC generation stations ......................................... 137

Figure 6.12 Architecture of the system under study ………..................................... 138

Figure 6.13 Active and reactive power in loads and generating units .......................... 139

Figure 6.14 Test case power system for integrating electric car storage system into AC grid

Figure 6.15 Frequency of microgrid regulated by DC side power management............ 141

Figure 6.16 Variation of DC generation to regulate the frequency of the AC grid ........ 141

Figure 7.1 Overview of smart grid testbed ............................................................ 142

Figure 7.2 Smart grid testbed laboratory (as of 9/25/2013) .......................................... 144

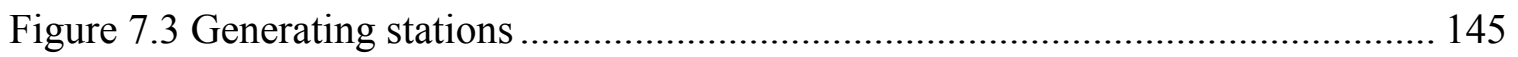

Figure 7.4 Synchronous generator and its accessories.................................................. 146

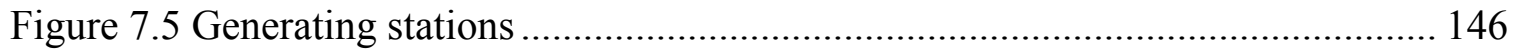




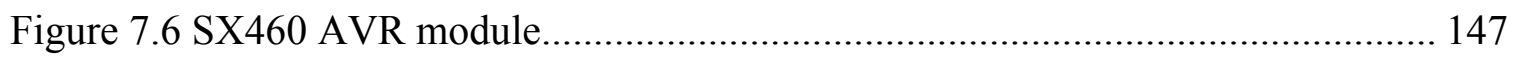

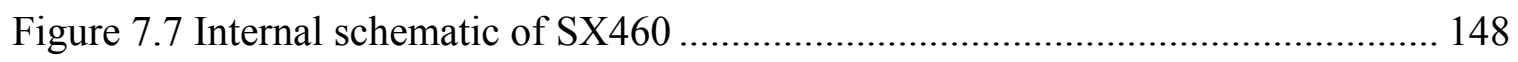

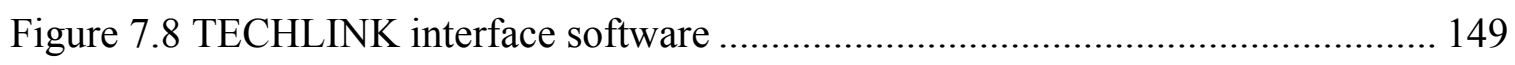

Figure 7.9 Schematic of a generator station control loop ........................................... 151

Figure 7.10 Developed transmission line model and schematic diagram of the 3 phase $\pi$-model................................................................................... 152

Figure 7.11 Bus model (a) Developed bus model with 3 feeders (b) Overview of each 3 phases input (feeder), (c) Electrical connection of CTs, (d) Electrical

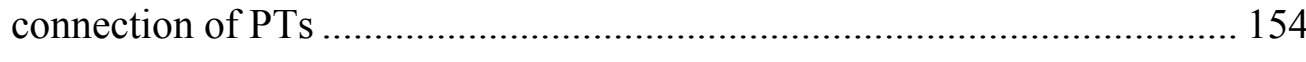

Figure 7.12 Schematic diagram of motor load emulator ………..................................... 155

Figure 7.13 Developed motor load emulator model .................................................... 155

Figure 7.14 Load box , (a) Each load box with 2 independent loads (b) Internal components (Resistors and switches) ...................................................... 156

Figure 7.15 Resistive load communication and connectivity scheme ........................... 157

Figure 7.16 Internal connection of each resistive load .................................................. 157

Figure 7.17 Inductive load box contains 2 individual loads ......................................... 158

Figure 7.18 Internal connection of each inductive load............................................... 159

Figure 7.19 Schematic diagram of the first control circuit for inductive loads .............. 160

Figure 7.20 PCB design and pre-manufacturing progress ........................................... 160

Figure 7.21 Inductive load controller equipped with XBEE and STM32F4 Processor.. 161

Figure 7.22 Inductive load box and control scheme ………........................................ 162

Figure 7.23 Simulink model for inductive load controller and communication circuit.. 162

Figure 7.24 Subsystem in load control module. .......................................................... 163

Figure 7.25 Manual load change software for inductive loads .................................... 163

Figure 7.26 Programmable load interface ................................................................... 164

Figure 7.27 Internal connection of synchronizer module ............................................. 165 
Figure 7.28 Developed synchronizer box ………………....................................... 165

Figure 7.29 Automatic synchronization logic........................................................... 166

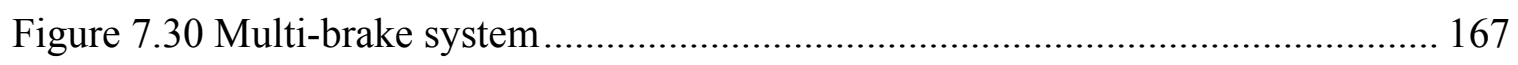

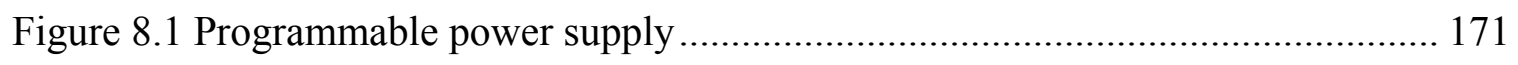

Figure 8.2 Photovoltaic power profile emulator software interface .............................. 172

Figure 8.3. Voltage-current and voltage-power curve of a photovoltaic module........... 173

Figure 8.4. Block diagram of a photovoltaic system with MPPT controller .................. 174

Figure 8.5. Wind generator electrical system: (a) DC grid connection (b) AC grid

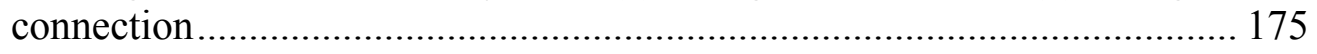

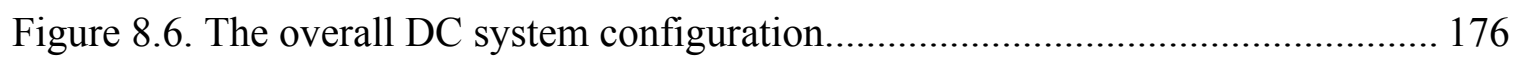

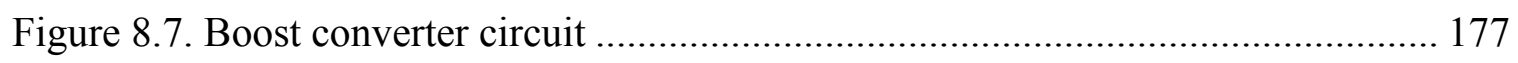

Figure 8.8. Experimental test setup for wind turbine and PV emulator. ........................ 177

Figure 8.9. Monthly average wind speed at La Venta station [107].............................. 177

Figure 8.10. Battery energy storage for frequency regulation in islanded power

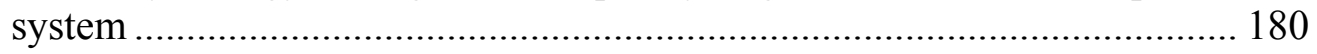

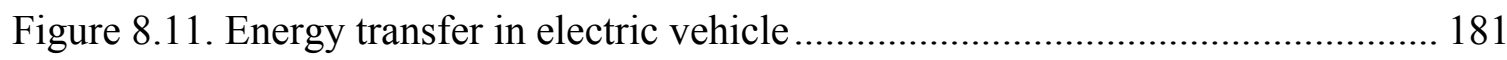

Figure 8.12. Schematic diagram of bidirectional energy transfer between two DC buses

Figure 8.13. Bidirectional buck boost convertor schematic and actual developed device.

Figure 8.14. Vector decoupling control of the bidirectional converter for power

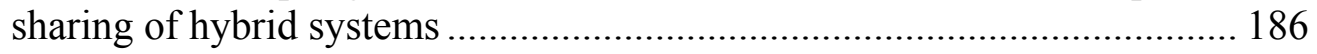

Figure 8.15 Developed grid-tied inverter in energy systems research laboratory .......... 186

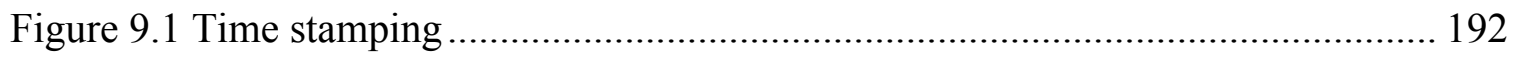

Figure 9.2 Total Vector Error ............................................................................... 193

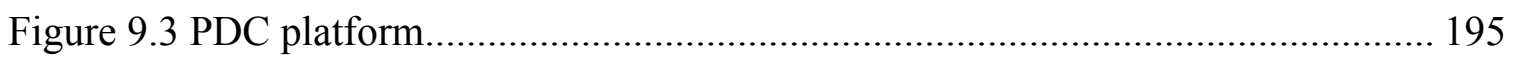

Figure 9.4 Common word definition ...................................................................... 196 


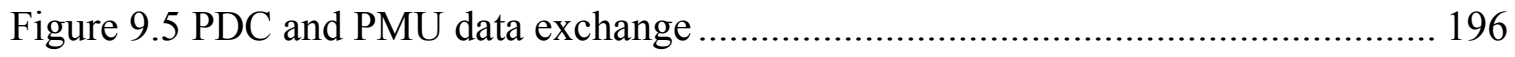

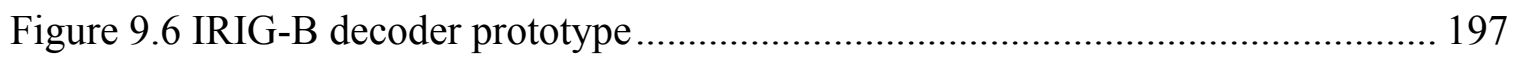

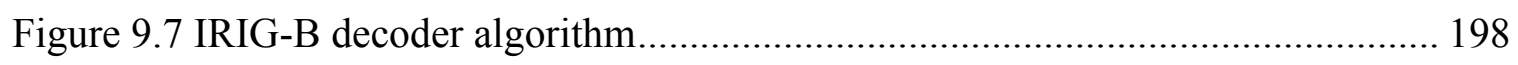

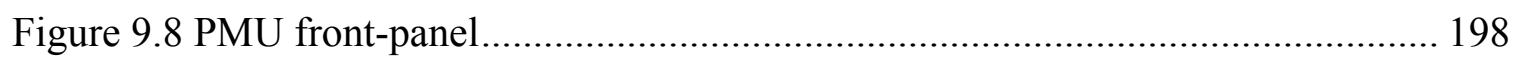

Figure 9.9 The software structure of the developed PMU ........................................... 200

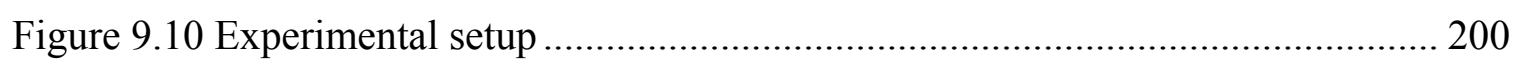

Figure 9.11 Integrated PMU and PDC setup with PLC and RTAC ….......................... 202

Figure 9.12 Measurement of the total delay in IRIG-B decoder module ....................... 203

Figure 9.13 Total Vector Error ............................................................................ 203

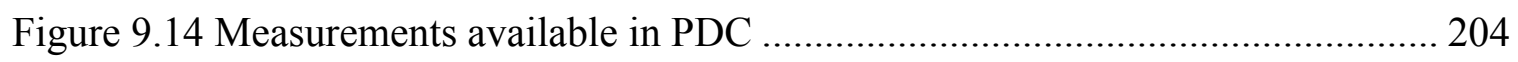

Figure 9.15 Comparison of phase angle measurements from SEL421 PMU and developed PMU without offset calibration................................................. 205

Figure 9.16 Comparison of phase angle measurements from SEL421 and developed PMU with offset calibration ...................................................................... 206

Figure 9.17 Comparison of voltage and rate of change of voltage from SEL421 and Developed PMU ..................................................................................... 207

Figure 9.18 Comparison of phasors and frequency measurements from SEL421 PMU

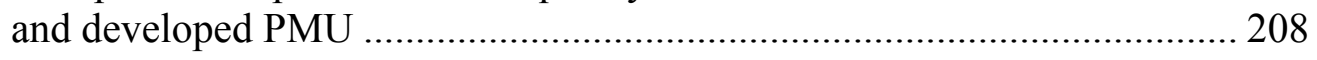

Figure 9.19 Captured waveforms by high resolution oscilloscope................................. 208

Figure 9.20 Enlarged area for green box in Figure 9.19 ............................................ 209

Figure 9.21 Enlarged area of yellow box in Figure 9.19 .......................................... 210

Figure 9.22 Enlarged area of red box in Figure 9.19 ................................................ 210

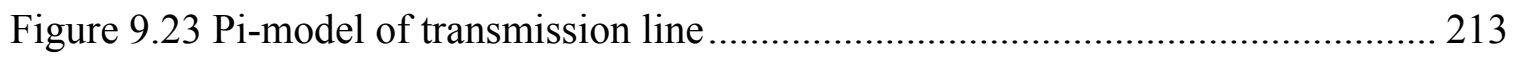

Figure 9.24 a) Voltage magnitudes b)Voltage angles c)Current magnitudes d)Current angles e) Active power f) Reactive power ................................... 213

Figure 9.25 Calculated resistance and reactance ....................................................... 214

Figure 9.26 The Calculated susceptance and conductance............................................ 214 
Figure 10.1 Islanding detection with PMUs located on both main grid and microgrid.. 221

Figure 10.2 Islanding detection with other PMUs within the microgrid ...................... 223

Figure 10.3 Microgrid simulation in Matlab Simulink.............................................. 224

Figure 10.4 Islanding detection with PMUs between main grid and microgrid ............ 226

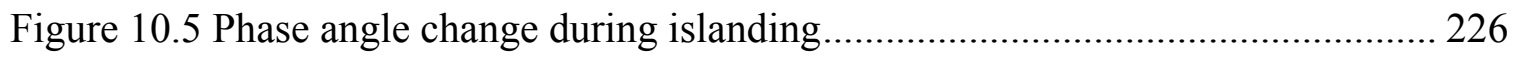

Figure 10.6 Changes of sequential RMS values in microgrid ................................... 227

Figure 10.7 Front panel of experimental test setup............................................... 229

Figure 10.8 Experimental results for 3-phase islanding (Time window is 60 secs.) a)

Bus $0380 \quad$ b) Bus 0260 ................................................................... 230

Figure 10.9 Unbalanced islanding at feeder 0260A............................................. 230

Figure 10.10 Changes of frequency during unbalanced islanding (Time window is 60 Secs.) a) Bus 0380 b) Bus 0260

Figure 10.11 Comparing sequential RMS and angles at bus 0380 and $0260 \ldots \ldots \ldots \ldots \ldots . . . .232$

Figure 11.1 Flowchart algorithm of the synchronizer developed in real-time software. 240

Figure 11.2 One line diagram of testing power system

Figure 11.3 Torque-Speed curve of prime mover generator a. for unloaded generator b. with local load at the terminal of the generator...

Figure 11.4 Duty cycled method, schematic of hardware connection 244

Figure 11.5 Fluctuation of generator speed in duty cycled torque controlled method (Before connecting G3 to the grid)

Figure 11.6 Fluctuation of generator speed in duty cycled torque controlled method when it has a local load at the G3 terminal (The torque is varying between 13 and $15 \%)$......

Figure 11.7 Dynamic load brake method schematic hardware connection ................... 246

Figure 11.8 Overall schematic of the emulated generator station and its measurements, communication and controller components ..................... 247

Figure 11.9 Block diagram and wiring of dynamic load ...................................... 248

Figure 11.10 Implemented circuit for dynamic load ............................................ 248 
Figure 11.11 Frequency (speed) control loop by using a dynamic load brake ......

Figure 11.12 Control loop for controlling the phase by changing the frequency set point.

Figure 11.13 Front panel of dynamic load to match the frequency of the generator

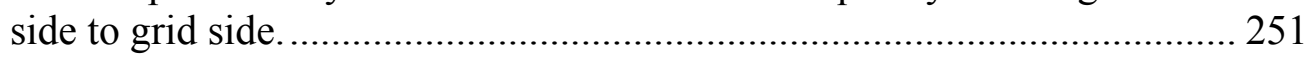

Figure 11.14 Background of dynamic brake VI ................................................... 251 


\section{LIST OF ACRONYMS}

\begin{tabular}{|c|c|}
\hline $\begin{array}{l}\text { ACRONYM } \\
\text { PMU }\end{array}$ & $\begin{array}{l}\text { DETAILS } \\
\text { Phasor Measurement Unit }\end{array}$ \\
\hline WAMPAC & Wide Area Monitoring, Protection and Control \\
\hline PLC & Programmable Logic Controllers \\
\hline NI-PXI & National Instruments PXI \\
\hline RTDS & Real Time Digital Simulator \\
\hline SEL & Schweitzer Engineering Lab \\
\hline EMS & Energy Management Systems \\
\hline TCB & Trusted Computing Base \\
\hline TSB & Trusted Sensing Base \\
\hline GPS & Global Positioning System \\
\hline PV & Photo Voltaic \\
\hline PHEV & Plug in Hybrid Electric Vehicles \\
\hline WAMS & Wide Area Measurement System \\
\hline BPA & Bonneville Power Administration \\
\hline CRC & Cyclic Redundancy Check \\
\hline SCC & System Control Center \\
\hline PSS & Power System Stabilizer \\
\hline PDC & Phasor Data Concentrator \\
\hline SCADA & Supervisory Control and Data Acquisition \\
\hline PMCU & Phasor Measurement and Control Unit \\
\hline HMI & Human Machine Interface \\
\hline
\end{tabular}




\begin{tabular}{ll} 
RTU & Remote Terminal Units \\
SE & State Estimation \\
WLS & Weighted Least Square \\
ADC & Analog to Digital Converter \\
VI & Virtual Instrument \\
DIgSILENT & DIgital SImuLation and Electrical NeTwork (calculation program) \\
IDS & Intrusion Detection Systems \\
HMAC & Hash Message Authentication Code \\
IRIG & Inter-Range Instrumentation Group \\
SPI & Serial Peripheral Interface \\
DAQ & Data Acquisition \\
UTC & Universal Time Clock \\
TVE & Total Vector Error \\
FE & Frequency Error \\
ROCOF & Rate of Change of Frequency \\
ESRL & Energy Systems Research Laboratory \\
RTAC & Real-Time Automation Controllers \\
FATCS & Flexible AC Transmission System \\
AVR & Automatic Voltage Regulator \\
EMI & Electro-Magnetic Interference \\
PCT & Current Transformer \\
UART & Potential (Voltage) Transformer \\
\hline
\end{tabular}




$\begin{array}{ll}\text { PPPE } & \text { Photovoltaic Power Profile Emulator } \\ \text { MPP } & \text { Maximum Power Point } \\ \text { PMSG } & \text { Permanent Magnet Synchronous Generators } \\ \text { DSP } & \text { Digital Signal Processor } \\ \text { SVPWM } & \text { Space Vector Pulse Width Modulation } \\ \text { LV } & \text { Low Voltage } \\ \text { HV } & \text { High Voltage } \\ \text { SPWM } & \text { Sinusoidal Pulse Width Modulation } \\ \text { RPEED } & \text { Reactive Power Export Error Detection } \\ \text { PLCC } & \text { Power Line Carrier Communication } \\ \text { PLL } & \text { Phase Locked Loop } \\ \text { DER } & \text { Distributed Energy Resource } \\ \text { EV } & \text { Electric Vehicle } \\ \text { G1 } & \text { Generator \#1 } \\ \text { IL1 } & \text { Inductive Load \#1 } \\ \text { NERC } & \text { North American Electric Reliability Corporation } \\ & \end{array}$




\section{INTRODUCTION}

\subsection{Introduction}

In recent years, there has been an increasing need to address new issues in the areas of global energy, security, economic growth and climate change. This would also mean addressing issues related to the stable operation of critical power infrastructures through the development of better techniques for the smarter use of hybrid energy systems including renewable and distributed resources.

According to BP's energy outlook [1], the population of the world has tripled since the 1900s, the income has grown 25 times, and the energy consumption has increased 22.5 time. About one third of the world's population have no access to electricity right now and this number is expected to rise to 3 billion by middle of the century according to Forbes. This is due to the fact that the world's population is expected to reach more than 9.3 billion by 2050, based on United Nations' forecast. Forbes also expects the demand for energy to be doubled by then, not considering United States' consumption. Meeting this increasing demand requires serious efforts that would involve major improvement for the future of the power systems. [1]

The energy demand is accompanied by a reduction of carbon emission, lower costs of energy for consumers, efficient production and operation with increased reliability, and economic advantages.

Smarter grid is the key, which can improve reliability, security, and efficiency of energy systems by enabling an optimal combination of renewable energy and energy 
storage in addition to conventional bulk energy resources. This integration is achieved with advanced information and communication technologies. It requires coordinated operational measured between consumers, utilities, and systems from generating units to end users.

The increased use of renewable energy resources coupled with the recent requirements for generation synchronization, real-time information availability, communication and cyber security, have initiated new research areas in the field of the smart power grids. The current structure of power systems has major deficiencies in realtime control and operation and it requires improvements incorporating innovative technologies.

With new developments in power grids, they are moving from the state of traditional bulk generation (Figure 1.1) to future grid with variety of components, participants, with lots of renewable energy resources in hybrid AC/DC grids, working close to full capacity and with a lot of sensitive loads (Figure 1.2).

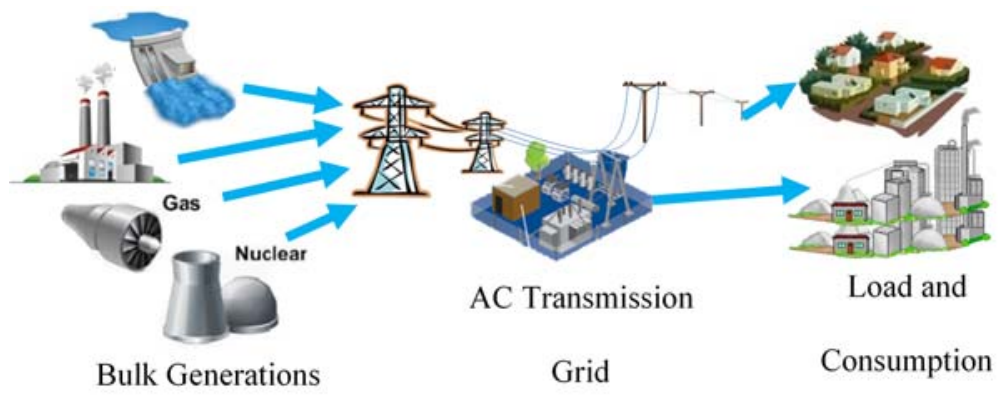

Figure 1.1 Traditional Power Grid structure

The move from old-fashioned measurement, communication and control to the edge of technology with computerized system, which has high speed controllers, better efficiencies and in general, higher performance. The increase in quality of the demand 
requires not only higher level of improvements, but also new ideas in order to satisfy the essential needs of incorporating multiple techniques in a variety of fields.

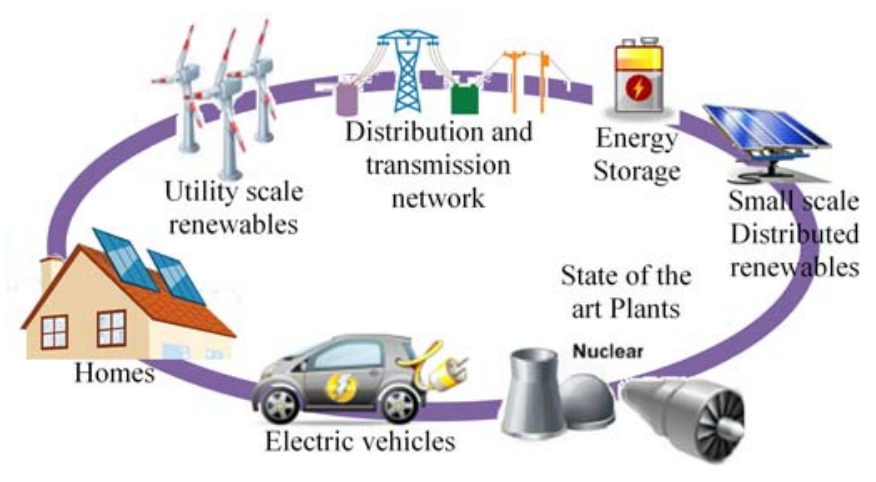

Figure 1.2 Future Power Grids

The power system has two major infrastructures; the power grid and the communication systems. It is a complex interacting system that needs to be understood for better realization.

Future power systems will involve various energy resources each with their different limitations and characteristics. By considering the future automation systems and intelligent participation of computerized components, the interaction between components may affect the of the power grid.

This will make the system even more complex. The smart grid has received considerable attention which resulted in numerous definitions such as:

- It is a modern electricity system, which uses sensors, monitoring, communications, automation, and computers to improve security, reliability, efficiency, flexibility, as well as the safety aspect of the electricity system. [1] 
- It is a class of technology, which people are using to bring bidirectional communication and computer technology into the power industry involving electricity networks to improve efficiency of electricity grid [2]

People may sometimes confuse the terms smart grid and smart metering. The metering is just a small portion of the smart grid and it is a technology involving two way communication, which can enhance the operation and can provide the consumer participation. The success of the smart grid depends on attracting and motivating a large number of consumers to be effective by using these kind of technologies as well as utilities to incorporate higher level of technologies in their cyber physical system.

There are many challenges which limit the reliability and efficiency of power systems. These can be summarized as the follows:

- Variety of participants in terms of operators, procedures and customers.

- Variety of components, which are in an interconnected, widely spread and distributed environment.

- Multi-layer operation of components, with different requirements, such as their burden, bandwidth, timing, etc.

- Unpredictable disturbances and faults, which can lead to wide spread failure instantly.

- Size of the system, which limits it from centralized monitoring and controlling in real-time operation.

- Intentional threats and vulnerabilities in the cyber physical environments.

The overall definition of smart grid is dreamed by the following attributes: 
- Intelligent: fully loaded with sensors and agents, autonomous, and fast response.

- Efficient: supply larger number of consumers without a physical infrastructure increase.

- Accommodating: capable of integrating new and renewable energy resources and storage facilities.

- Motivating: enabling dialog between consumers and utilities.

- Opportunistic: create new markets for supporting plug and play operation.

- Quality focused: capable of delivering high quality power to consumers.

- Resilient: resistant to attacks and natural disasters.

- Green: support global climate change by creating significant environmental improvements [3].

These are the seven properties which the U.S. Department of Energy required for the next generation of the power grids. Smart grid technologies will be used to facilitate an array of sub-areas in the field of electric power including generation, transmission, distribution, and marketing. Most of these improvements are made by advancements in communication and information technologies.

\subsection{Current State of Power Grids}

\subsubsection{Smart/advanced metering infrastructure}

Deploying smart meters at consumer locations with the goal of reducing costs and increasing the reliability by integrating the distributed generations will the electricity distribution. Smart meters help the consumers with smooth control over their energy 
consumption and also energy generation by their local energy resources. It also helps the utilities to detect the power outages remotely, read the measured consumptions remotely, and offer some extra options to the consumer, as well as demand side management options.

\subsubsection{Wide Area Monitoring, Protection and Control (WAMPAC) Systems}

Wide area measurement systems are mostly based upon Phasor Measurement Units (PMUs), which provide accurate GPS-based timed sampling and enables synchronized readings of the states of the system. Providing these information to utilities and regulators will empower the grid with high potential of new applications. The development of advanced control applications depends on wide area measurements and it is expected to be an important element of future power grids.

\subsubsection{Operation and Automation}

New communication infrastructure and algorithms are recently integrated to improve the reliability and performance of inter-substation devices. Old substation usually use serial or analog communication techniques, but new substations utilize Ethernet networks, which enable multicast transmission of information, provide real-time awareness of all substation events, and helps the operational of the protection scheme. The digital technology has already revolutionized communications across utility networks and the same techniques are being applied within the substations to enable fast and responsive messaging over the Ethernet and fiber optic channels using the IEC61850 standard. 


\subsubsection{Information Technology and Communication Improvements}

Technology weaknesses exist in the communications technology, devices, and software applications used in the automation industry. It is the process of ensuring that the power system is designed to operate at a level of security that is consistent with the potential harm that could result from the loss, inaccuracy, alteration, unavailability, or misuse of the data and resources that it uses, controls, and protects.

In order to ensure transfer and interoperability of the information required to support the grid, common information models are being used increasingly in the smart grid [4]. They have been primarily developed in order to assist system integration through consistent data representation and exchange formats.

\subsection{Motivation for Solving the Existing Problems}

As mentioned earlier, smarter grid, together with renewable energies, has received much attention to address challenges in global energy demand, security and improvement among energy consumers (residential, commercial and industrial), vendors (providing technology, service, etc.), utilities (including generation plants, natural gas suppliers, marketers and power providers), governments (including federal, provincial and municipal levels) and R\&D institutes.

For better environmental outlook, government always searching for new ways to reduce the carbon emission, meet the increasing demand for energy, promote energy conservation and eliminate dependency on fossil fuel. At the same time, consumers are looking for a reliable energy supply and reduced costs. Utilities also want to improve their system reliability and reduce the energy production costs as well as participate more 
in new energy businesses. Most of these companies want to increase electrification, replace or improve coal power plants operation and increase distributed generations along with renewable energies.

Given the multi-faceted nature of the technical benefits achieved through implementation of the new approaches discussed here, the research in this dissertation will cover an array of different yet related subjects associated with the cyber-physical property of smart power systems. Some of these subjects are as follows:

\subsubsection{Essential applications are in practice}

The techniques developed here will be verified on a Smart Grid Testbed with various test facilities including real-time cyber-physical platform. This provides a great opportunity to model, design and create samples for variety of power system components with exclusive application and characteristics. This Smart Grid Testbed involve facilities of subsystems such as power electronics, energy storage system, bulk generation along with renewable energy resources, communication, identification techniques, processing and analyzing tools and also synchronized measurement systems.

\subsubsection{Real-time analysis and operation}

Integration of multiple microgrids in the smart grid requires an infrastructure to design, test and verify ideas to control/improve the system in a real-time closed loop system. For this purpose, this research builds on the fact that a hybrid power system involving real-time control and microgrids can operation through hardware in the loop (HIL) systems. This is because microgrids need measurements and parameters to be shared with other sections of the physical system through the communication infrastructure. Real-time operation and physical infrastructure of emulated power system 
enable researchers to experimentally run scenarios and investigate hidden issues in virtual systems.

\subsubsection{Verifications of developed techniques on hardware based testbed}

The advantages of having a hardware based test setups are as follows:

- Compare different solutions and approaches in the existing power system.

- Faster and reliable results due to availability of actual power system model and components.

- Solving the problem in presence of actual environmental conditions, such as noise, none ideal conditions as well as hidden or neglected factors which may be concealed in simulation-only techniques.

- Identification of factors when accomplishing the solution through replication of experiments.

- Providing a training environment not only for research but also for training and preparing users.

\subsubsection{Simulation systems with physical conditions, cyber security and interoperability}

By having communication/cyber infrastructure along with field components, a complete architecture of an integrated Information Technology (IT) and power system can be monitored, managed or revised for specific scenarios, such as communication security threats. The communication infrastructure reacts this instant of data initiation (which is the transducer) to the instant it reaches the control center. Analog and digital flow of data are available and security compromises can be studied directly in each 
category. Technical difficulties in implementations of solutions makes this research much applicable, challenging in addition to being functional.

\subsection{Literature Review}

According to challenges which were described in the introductory section above, the current power systems' operation and control require innovative methods and techniques in order to enhance the performance and also optimal functioning of the system [5], [6]. In terms of digital information technology, to create more efficient and reliable grid, which is responsive to disturbances, future grids needs to be appropriately managed and controlled [7]-[9]. References [10]-[13] describe the future of power systems and their road map. Smart grid can provide capabilities, such as predicting system behavior, anticipatory operation and adaptation to new environments handling distributed resources, stochastic demand and optimal response to smart appliances [14]. In the smart grid area, there are particular fields which need to be studied by developing real-time practical or operational solutions [7], [10], [15]-[24] such as:

- Phasor measurement units and their practical applications in power systems for improved monitoring, protection, and control.

- Integration of distributed energy sources including renewable resources (wind, solar, fuel cell, etc.) in power generation and their fully controlled structure.

- Intelligent protection schemes and their application in detecting, mitigating, and preventing cascading outages, islanding situation, and total grid blackout occurrences. 
- Integration of hybrid AC-DC systems by creating new microgrid solutions for residential and industrial applications.

- Real-time or dynamic energy management and demand side management based on renewable uncertainty.

- Reactive power support and distributed voltage control, remote control of FACTS and HVDC devices to enhance overall system performance and islanding detection of a local and wide area power system and develop proper separation actions.

Based on [6], [25], [26], the integration of modern communication infrastructure has improved the reliability of power grids in the wide area power systems. It has also improved the operational efficiency, security and safety of these systems. Fast data exchange among system components and control centers made the huge enhancement in wide area applications [9], [27]. This is mostly initiated by PMU integration, which was first developed by Phadke et al. His work not only include the development of PMUs, but also extends into placement, calibration, communication requirements and applications of PMUs [18], [28]-[38]. This work is accompanied with the works of Thorp et al. who also integrated the state estimation improvements techniques [39]-[45]. Abur et al. also presented large investigations in the field of state estimation for application in power systems. Although state estimation can improve the security of information, it may also be the target of cyber-attacks. In [46], the cyber security aspect of state estimators in supervisory control and data acquisition (SCADA) system for operation of power grids was analyzed. 
The concerns coal depletion inspired the use of renewable resources [47]. Integration of DC resources, storage systems and centralized load and generations led to more incorporation of micro grid concept, which usually have only one point of common coupling with the main grid, and can function autonomously [48].

Dealing with wide spread renewable energy resources and storage systems available in Plug in Hybrid Electric vehicles (PHEVs) in the future power system, which led to microgrids. Interoperability of such systems needs to be studied and improved using the latest improvement in communication and information technology, such as PMUs. Islanding detection can be one of the other applications of PMUs. Although many researchers have already investigated the islanding detection techniques [49]-[51], there are recent improvements in this field using PMUs [11], [52]-[54]. They have concentrated on complete islanding detection techniques, based on phase and frequency measurements, but they have not studied the situation when only one phase of a multiphase microgrid is disconnected from the main grid.

According to [20], the need for smart transmission grids can be summarized into four aspects: environmental challenges, market/customer needs, infrastructure challenges and innovative technologies.

Smart grids consist of significant cyber components that manages the electric power infrastructure[12]. They need to be operating safe and reliable and this is a large concern for our society and a many organizations involved in maintaining the grid safe and secure operation [55]. Although SCADA and substation automation is well covered by advanced standards such as IEC61850 [56], there are significant number of recent studies, which are departing from centralized SCADA type control into distributed and 
agent based control and operation schemes. This is done in order to follow the North American Electric Reliability Corporation (NERC) critical protection scheme, which needs to protect the power system infrastructure from both cyber and physical attacks [57]. For this purpose, some works address the development of a real-time testbed by integrating Allen Bradley Programmable Logic Controllers (PLC), National Instruments PXI (NI-PXI) controller, Real Time Digital Simulator (RTDS), and Schweitzer Engineering Lab (SEL) devices [58]. Another study was conducted on the testbed of University college Dublin regarding the cyber security issues in the smart grid environment [59]. Although this is a complex testbed, it cannot provide the required procedure in terms of hardware integrations and renewable resources. It follows the software simulation platform and this is not sufficient for a cyber-physical platform.

By addition of synchrophasors to the existing power grid, the real-time monitoring and analysis of the grid is enhanced. This relies on communication network infrastructure and cyber security vulnerabilities which must be addressed to ensure the stable, secure and reliable operation of the system. It is also suggested to have a network on synchrophasors the deal with the security concerns [60], [61].

Comprehensive monitoring of the power grids require trusted sensors; otherwise, adversaries may exploit the introduced attack surfaces to cause false data injection attacks [62]. In particular, attackers compromise and take control of a specific subset of the power system sensors, e.g., phasor measurement units (PMUs), and corrupt sensor measurements such that they could evade the state estimation procedures within the control centers. The cyber-side diagnostics mechanisms try to estimate the security state of a computer network [63]. Ten et al. [64] proposed a vulnerability assessment technique 
to evaluate the vulnerabilities of SCADA systems at three levels: system, scenarios, and access points. Mohajerani [65] introduced a method to detect and improve the vulnerability of power networks against the intrusion and malicious acts of cyber hackers by calculating the risk of each asset being compromised. Both the abovementioned frameworks perform in an offline manner, and hence cannot monitor the system for malicious activities while it is in its operational mode. Additionally, there have been several solutions introduced to provide security to the substation-control center communication links [66]. For instance, Kang et al. [67] suggest an end-to-end encryption and key management solution for power grid infrastructures. Almost all of the previous solutions to employ cryptography to guarantee the overall infrastructure security fall short in two major aspects. First, it is practically hard to perform data encryption and decryption on measurement samples in real-time especially in large-scale power grid infrastructures with large amount of network traffic. Second, as the encryption is performed in substations after the measurements are sensed and sent out to the analog-todigital devices, the proposed solutions are not resilient against analog-to-digital device compromises.

\subsection{Problem Statement}

The operation of power systems with microgrids has become significantly complicated with high penetration of renewable energy resources. The occurrence of connection and disconnection to utility grids may introduce vulnerability cases in the power system as cyber-physical infrastructure, market operation, and demand side management. The scheme for power sharing between AC and DC microgrids and their 
interaction needs to have operational control strategies. Therefore, the development of advanced Energy Management System (EMS) for hybrid microgrids is necessary. From operating point of view, EMS should be able to have real-time measurement, correct information, and a monitoring scheme to optimally control it and finally, achieve a stable and economic reliable system. From component view, it should be fast dynamic, accurate and satisfactory in critical situations. The goal of optimum, stable and secure operation of power systems requires correct control and operation between components and the EMS. , This requires trusted and correct data through a communication interface between the components and the EMS. One of the enablers for improving the quality of energy systems is the synchrophasor technology. Although, there are PMUs available in the market, the potential for improvement of their features and specially their application is needed for real-time studies. Throughout the move toward the smart grid, several concerns regarding the security in operation of future powers systems required to be addressed. Regarding the measurement accuracy, and trustworthiness, although state estimation techniques are developed in traditional networks, they still can be greatly improved by incorporation of PMUs to change the state estimation to state measurement. Using synchronized time stamping not only helps the measurement system, but also they have the potential to be integrated in authentication and security systems as well. Developing such improvement include both the cyber and the physical aspect of the power system, hence it requires experimental real-time verification to insure the interoperability of the intended methodologies. 


\subsection{Purpose of Research}

Power grids are critically dependent on reliable information from sensing points, so this technology can work on two opposing sides. On one side, it supports the system monitoring, measurement, control, operation and reliability. But on the other side, it opens a window of deficiency of power grids due to lack of security. It lets external agents with malicious purposes to cause planned events with catastrophic consequences.

In addition, with large penetration of renewable energy sources and expansion of AC/DC microgrids requires energy management systems (EMS) to perform various control and operating tasks close to real-time with high reliability and security especially in cases of contingencies and critical situations. Wide area monitoring, protection and control (WAMPAC) systems offers a cost effective solution to improve system planning, operation, and real-time control. This is because of synchronized measurement technology that is an important element and the enabler. There are a number of platforms and tools for synchronized measurement systems, such as data acquisitions, data concentrators, phasor measurement units, SCADA/EMS, and market operations. There are two key applications categories that can benefit from the synchronized measurements:

1. Analysis and prevention of outages that may appear in blackouts

a. Due to the fact that they are accurate, have wide area coverage and can track the power system dynamics.

b. They can provide true early warning systems and criterions to detect critical scenarios and can help in restoration and quality of information for event analysis.

2. System operation and control 
a. State estimation solutions can improve pricing calculations, overall accuracy calculations, and energy strategies can be improved as well.

b. System margin management can be assisted and real-time knowledge and awareness of actual system conditions can be increased.

This dissertation is inspired by the need for:

1. Extended hybrid (AC/DC) grids and microgrids.

2. Their need in the field of cyber-physical security

3. Measurement technology, communication and automation systems

4. Wide-area control and operation

5. Microgrids incorporation and renewable energy resources integration

This dissertation is will introduce methodologies for a more reliable and autonomous operation of power systems, by integrating security improvement, data validation, and synchronized measurement technology and its applications in the Wide Area Measurement Systems (WAMS).

The introduced studies and methodologies need to be verified in a practical environment. Therefore, they require integration on a practical cyber-physical real-time hybrid platform which is developed as the smart grid testbed. It involves variety of renewable energy resources and distributed loads. This purpose of this dissertation is to mainly improve the overall power system performance through the enhancement of measurement systems, synchronization techniques, and cyber-physical security for real- 
time operation of smart grids and also to verify the proposed methodologies on a unique experimental platform.

\subsection{Original Contribution of This Dissertation}

The original contribution of this dissertation is to develop techniques for widearea monitoring, real-time control, secure and stable operation of power system including large penetration of renewable energy resources and corresponding microgrid structures. In addition, this dissertation provides a platform named the smart grid testbed infrastructure in which developments, integration and verification of several principles for real-time, hybrid, and critical power system situation can be performed.

The physical system includes generation emulation through several generating stations equipped with synchronous generators with total capacity of $36 \mathrm{KVA}$ in $\mathrm{AC}$ system and similar amount in DC side through programmable power supplies which emulate the solar cell behavior and wind farm emulators. Produced energy will be distributed through several cable and transmission line models equipped with protection fuses and data acquisitions for measurement and switching purposes. Combination of the AC and DC supplies along with several designed programmable load modules can provide a close to real world power system, which has microgrid integration capability, flexibility of different architectures involved with actual challenges of real world conditions. This system also has a software environment to monitor the system parameters and apply control, analysis techniques similar to a HIL system.

Real time analysis of power system scenarios and their implementation on the testbed is a unique feature, which makes it exceptional from real-time simulation-only 
software packages and has capabilities to perform both research and training objectives users. In addition, the integration of commercial hardware for evaluation, development and improvement purposes can also be conducted.

The developed SCADA center monitors the system with close to real-time awareness (less than a second snapshots) and has connection to power system analysis software packages. The software base for this system is the LabVIEW environment which includes the human/operator interface and connectivity to other commercial modules such as the Digsilent Power Factory software as the real-time analysis environment. To enable bad data detection and finding the actual state of the system, is the testbed is also equipped with developed power system state estimation and real-time system monitoring feature.

Since synchronization of dispersed and most probably small generating stations are occurring more often in future power systems, a new device was developed called Dynamic Brake, which helps the process of synchronization to be faster and more reliable.

This is along with penetration of phasor measurement units and it is needed to establish a phasor measurement prototype for application in testbed, along with the available commercial systems. This opens the field to integrate the idea of synchronized measurements in variety of applications. Phasor measurement technology is investigated not only in integration and implementation issues in terms of accuracy and operation but also will be used toward many other applications. One of them is islanding detections in microgrids, and the other can be transmission line parameter identification. This dissertation also developed a laboratory based PMUs as a tool for detecting partially 
islanded microgrids. This is quite novel and completely developed by the author and tested in experimental platforms and its performance was verified with commercial PMUs.

Moreover, we integrate phasor measurements along with our SCADA system to provide a state estimation procedure for future applications in system operations.

The technology of synchronized measurements has also been used to improve the security of power grids by introducing an original approach named Trusted Sensing Base (TSB) which has unique features and uses the GPS time synchronization technology along with digital scaling for securing the analog measurements of sensors in future power systems.

For synchronization, data acquisition, communication, control, and security of the system innovative solutions are offered and several devices and modules were designed, built and successfully tested. Their application was also verified and they will be exclusively introduced within the next chapters of this dissertation.

\subsection{Dissertation Organization}

This dissertation is prepared in twelve chapters including the current chapter and they are organized as the following:

Chapter 1 includes the introductory discussion on advancements and improvements in the current power systems and moving toward the next generation of integrated technology in power system. It provides a literature review including the recent developments, needs and requirements in improvement of power systems. In addition to the introduction, it explains the purpose and motivations of this research and 
express the problem statement with the requirements for this research. A brief enumeration of this dissertation contributions is included.

Chapter 2 express the futuristic needs of power systems operation and their future look in terms of renewable energy resource integration, information technology, communication and security. The recent developments in communications and integration of phasor measurement units provides a variety of innovations in the field of smart grid.

Chapter 3 introduces the SCADA in power systems and extends the concept into their real-time operation and implementation requirements. It also discusses the software platform, which is used for real-time operation, integration of developed techniques and interconnectivity of developed modules available in this system under a unique project. It discusses the details of the LabVIEW project through its Virtual Instruments (VIs) and sub-VIs. These are needed to work in a modular structure, so can be extended based on the individual requirement for each purpose. These modules include our SCADA and online system monitoring, developed measurement calculation functions, synchronization procedure, data alignment, and data publication for archiving or monitoring purposes, etc. In this chapter each of these modules will be explained individually but under a coherent structure.

Chapter 4 introduces the state estimation (S.E) as a method to improve the suitability of measured values and estimation of non-measured values as well as integration of real-time state estimation technique in the smart grid testbed verification platform. This chapter also presents the developed S.E. program and the real-time interface for third party S.E. software. This chapter also introduces the latest 
developments in the field of state estimation with incorporation of PMUs, and develops a new technique, for several hybrid S.E. cases, when several PMUs found identical time deviations.

Chapter 5 introduces a state of the art technique for securing the critical power system structures through "Trusted sensing Base" (TSB). This technique uses a GPSsynchronized encryption technique at the furthest side of the cyber space (which is the sensor location), and converts the scale of analog signal of voltages/currents in order to make them secure but also practicable for regular applications, such as PMUs. The threat model, high-level architecture of solution, the encryption technique for analog sensors will and finally the implementation of this system with hardware specifications and technical considerations will be fully explained. To show the applicability and to verify the methodology, several evaluations including time and accuracy analysis will be reported.

Chapter 6 explains the details of integrated microgrids in the smart grid testbed. They can be integrated in several modes of operation. The hybrid system will have the interaction of AC and DC sides and several scenarios and studies will be given in this chapter. The examples include AC grid studies, DC microgrid integration, internal power flow of $\mathrm{AC} / \mathrm{DC}$ systems, frequency controlling of $\mathrm{AC}$ grid by $\mathrm{DC}$ storage, and voltage profile improvement through reactive power compensation provided by DC microgrids.

Introducing the abovementioned techniques require hardware verifications, and in Chapter 7 the hardware implementations to provide a power system platform for such a purpose will be given. It incorporates several generating stations and introduce their characteristics of the design and operation along with the other grid components, such as 
synchronizer modules, transmission lines, and buses. It also identify all the developed load modules along with the measurement system, data acquisitions and the communication requirements.

Chapter 8 focuses on development and implementation of renewable energy resources, such as Photovoltaic (PV) and Wind. The hybrid power system, which is developed in the testbed, includes not only the AC grid but also different renewable energy emulators as well as energy storage systems involving batteries and ultracapacitors. The techniques for control and operation of each module (including bidirectional converters, power flow controllers, frequency controller of islanded microgrid, reactive power compensator) will be explained in the next chapter.

Chapter 9 introduces the phasor measurement unit's as a component which will be used in the future power systems. It also includes the definition and presents the development of a laboratory-based PMU and its connection and data transfer to PDCs. It introduces a complementary developed device named "IRIG-B Decoder" which provides the timing information for time synchronized power system applications. Several topics regarding the properties, accuracy and characteristics of developed PMU will be given and compared with commercial devices from SEL and available in the Smart Grid Testbed. In addition to this comparison, an accuracy analysis for measurements of the developed PMU will be carried out based on true time domain measurements of phase and frequency.

Chapter 10 introduces situation when microgrid turns into an islanded operating mode. There are several islanding detection techniques introduced hereby, including the methods using phasor measurement units. It includes simulations in different scenarios 
and introduces a new technique for detection of partially islanded microgrids, in which traditional methods are not capable of properly functioning and detecting such a condition. An innovative technique including PMUs was used in this study and the new technique was verified by simulation and experimental tests.

Chapter 11 discusses the synchronization process in the $\mathrm{AC}$ grids, when a generating station needs to be connected to the main grid and starts power sharing. The requirements and criteria for this procedure will be identified. A new automated system, which is named "Dynamic Brake", will be introduced. It facilitates the generation unit synchronization through the control of a dynamic load at the generator's terminals. Experimental tests prove the capability of the introduced method for fast and reliable synchronization procedures.

Chapter 12 summarize the dissertation outcomes, concludes the significance of this research, discuss the results, and finally makes recommendations for the future works. 


\section{REAL-TIME OPERATIONAL ISSUES OF POWER SYSTEMS AND REQUIREMENTS TO ACHIEVE SECURE OPERATION}

\subsection{Future Power Systems Need Higher Security}

The main factor contributing to catastrophic failures, such as blackouts, includes stressed state of power system components, specially the generation units and the transmission system. In such situation, which the generation has a minimum reserve, the transmission system operates close to its limits and reactive power supply is insufficient, therefore, a minor fault can become avalanche cascading event.

The lack of sufficient real-time measurements and analytical tools, in addition to incorrect and false tripping of relays, may worsen the situation, and finally, may lead to instabilities, which can split the grid into islands of excess load or generation, and eventually collapses the region into a blackout. The current power system is vulnerable not only to natural disasters, but also to security vulnerabilities and intentional attacks. The number of documented cyber-attacks or intrusions worldwide are rising rapidly in recent years. Because power systems completely rely on computerized communications and control, system security has become increasingly dependent on protection of the integrity of the associated information systems. A part of the problem is from the existing control systems, which were originally designed to be used by exclusive stand-alone communication networks, where in later, will be connected to the Internet (because of its productivity advantages and lower costs) but without adding the technology needed to 
make them safe and secure. Moreover, numerous types of communication media and protocols are going to be used in the communication and control of future power systems.

The operation of a modern power system depends heavily on a complex system of sensors and automated/manual controls; all of these are tied together making it a cyberphysical system.

While the direct physical damages to generators, substations, or power lines may be the most obvious strategy for causing blackouts, there are some activities that compromise the operation of sensors, communications, and control systems by spoofing, jamming, or sending improper commands or data could also mess the system up, and maybe cause blackouts. In some cases, these activities result in physical damages to keycomponents of the system. As a result, due to the potential of such threats, cyber-security is just as important as physical security and it is one of the priorities and essentials of smart grids.

In power systems, data perspective consists of data acquisition, transmission and processing along with reliability and security of the communication. Bad data, which comes from misconfiguration or malfunction of measurement units, may impact the operation or even the stability of the system and may result in severe results, as mentioned before the blackouts. It may also be initiated by cascading coordinated communication errors and may lead to global failures in the system. On the other hand, there are always threats by the attackers in order to manipulate or misconduct the process and hence security becomes more visible in such applications. 


\subsection{Future Power Systems are Hybrid and Involve Renewable Energy Resources}

Today's power systems are mostly composed of traditional fossil fuels in particular about $40 \%$ is coal, $25 \%$ is natural gas, $20 \%$ is nuclear energy, and only $15 \%$ is from renewable energy resources. Integration of more renewable energy requires advanced management solutions. [1]

Power generation and demand should keep their balance in real-time for the secure operation of the power grid. However, mostly during the peak load times, the supply becomes limited and the system operates on its boundary edges with a lower system reserve margin.

Due to the surge in demand, more renewable energy will be connected to the power grids every day. DC microgrids are getting more familiar nowadays, mostly because of the deployment of the DC components, such as Plug in Hybrid Electric Vehicles (PHEV) and renewable DC resources. The availability and advantage of storage systems available in DC microgrids is another inspiration for integration of DC networks. One of the most important features of the smart grid is its advanced structure, which can include connections of various AC and DC generation systems, storages and loads, with optimal asset utilization, efficient and reliable operation without failures. The operation of the grid is becoming more challenging, by considering the mentioned points, and it requires new and innovative methodologies in order to enhance the performance of the power system [5], [6]. 


\subsection{Future Power Grids Require Higher Level of Information Technology and Wide Area Communication Systems}

The operation of a modern power system depends on complex systems, all of which are tied together through the communication system. The communication system is growing fast and involves a lot of complex and modern developments.

Communication requirement for different applications are not the same[68]-[70], but there are some common important features in the Wide Area Measurement System (WAMS) networks, which are listed here:

- Reliability: Power systems have a large number of sensors, and they are increasing every day, the need for an accurate and reliable measurement system is increasing. PMUs are one of the best solutions and have high weight compared to other measurements, which are used for state estimations, as well as control and protection. They also can improve the reliability of communication network for the WAMS.

- Real time response: After the accuracy, the transmission timing is critical for most applications. In some cases, delays more than several milliseconds abort actions, such as fault protection, load shedding, or islanding. This latency makes the execution ineffective and hence, the real-time performance become a vital parameter in the WAMS networks.

- Security: If the communication network is unsafe, it can be attacked easily and the power grid can be destroyed eventually. So the health of the entire grid is depending on the safe operation of the WAMS. 
- Scalability: In today's continuous restructuring power networks, it is required for power grids to expand and merge systems based on the fresh needs. Accordingly, they need to have a scalable communication network in addition to an upgrade in the communication protocols and the transmission medium conveniently and economically.

Nowadays, with recent developments in information technology, WAMS consist of numerous measurements and a widely spread communication infrastructure. Their operational framework expanded to include measurement, monitoring, and control across the continent. Smart grids have particularly 3 challenges for implementation and application of communication systems: interoperability, unlicensed radio spectra access and cyber security [71].

Electric utilities are investing a lot in telecommunications and provided innovative communication networks for various purposes, such as real-time monitoring, control, operation, and protection. The use of broad-band networks lead to remote realtime applications called wide area monitoring, protection and control (WAMPAC).

The high speed communication is exceedingly desirable, especially for the realtime operation of power systems, which can improve their services and reduce the costs. Applications of such high speed and broad-band networks can be summarized as:

- Data acquisition from any point (generation, transmission, distribution)

- Hierarchal access to different levels of the data from homes up to control centers.

- Integration of weather services

- Generation control 
- Load shedding and contingency analysis

- Remote control of power equipment, such as FACTS devices and relays adjustments

Traditional SCADA/EMS, such as quasi-steady-state calculations, nonsynchronized data acquisition, and low data transmission rate made it impossible to sample the global state of the power system in real-time.

The main enabler of WAMS is phasor measurement unit (PMU) technology. Its information solves the problem of phasor quantities for a wide area of distributed substations. A typical WAMS includes PMUs, PDCs, centralized super PDC, and a communication network. The potentials of the synchrophasor technology improves power system communication integrity through wide area monitoring and control in order to create a robust, widely available, and secure synchronized data measurement infrastructure for interconnected power systems.

\subsection{Synchrophasor Technology is Broadly Spread in Future Power Systems}

Because of the large increase in the deployment of Phasor Measurement Units (PMU), synchronized measurement techniques are becoming more routine and widespread. This creates challenges for the utilities in order to come up with application difficulties and complexities. Although it enables the operators to be more convenient, in terms of available data, but at the same time may push to them to a complicated system with more sensitivity to malfunctions.

Synchronized phasor measurements are becoming an important element of the WAMSs and being used in advanced power system monitoring, protection and control 
applications. The PMU technology provides time-stamped measurements with 1-2 microsecond accuracy at the measuring point, so that the data transmission latency is no longer a critical parameter in using this data. The Global Positioning System (GPS) provides this high accuracy time reference for all measurement devices. It is agreed in literatures that PMUs have elevated the standards of modern power systems to a new level. The importance and applicability of the PMUs impact the number of the installed PMUs all over the world. Several major utilities have recently shown a noticeable interest in synchronous phasor measurement technology. These include hydro-Quebec (one of the largest utilities in Canada), American Electric Power, Electricity de France, Bonneville Power Administration (BPA). Furthermore, in China, most ultra-high and high-voltage buses are all equipped with PMUs.

PMU can provide power system operator with synchronized measurements for positive sequence voltage and current, phase angles, local frequency, rate of change of frequency, harmonics, negative and zero sequence quantities as well as digital or analog measurements. These data can be used in different applications in order to improve power system performance.

The first PMU was built at power system research laboratory at Virginia Tech. University [72], [73]. The structure and the main components of a PMU is depicted in Figure 2.1.

The information of the phasor is time tagged in the PMU and is sent to PDC. Then, PDC checks the information error by cyclic redundancy check (CRC) and afterward, rearranges the measurements, which are recorded by different PMUs, and 
aligns them based on their time reference, and finally, sends them to a shared memory/data base/archive.

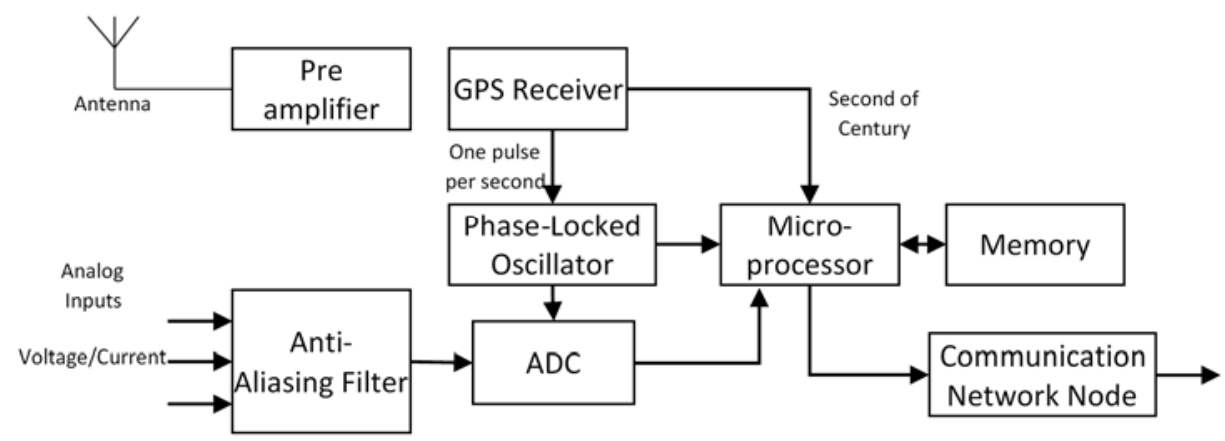

Figure 2.1 PMU structure

Super PDC is usually located in a data center, phasor data processing center or system control center (SCC) and most of the monitoring functions, the protection schemes, and the control strategies are executed by SPDC.

PMUs are mostly using satellite communication technology. The satellite communication can provide unique services, while are hard to attain from traditional /regular communication methods. The main benefits are:

- Remote monitoring of dispersed locations.

- GIS and earth imaging,

- Highly accurate and continuous time synchronization and location tracking

- Wide bandwidth and range of applications for satellite based data communication.

Global Positioning System was initiated by US Department of Defense in 1978 and covered worldwide for the position data in both military and public applications. It 
consists of 24 satellites, in six different orbits, which rotates every 12 hours around the earth with the distance of about 11000 miles. It is designed in such a way that at least 5 of them can be viewed from any location on earth. There are 5 monitoring stations and four ground antennas located around the world to gather the information to the master control station, which is located in Schriever Air force in Colorado. This center provides the correction commands/actions and send them to the satellites.

A GPS receiver can be located anywhere. It can locate the satellite and its distance as well as latitude, longitude, and altitude. By having such information, it can calculate the speed, sunrise and sunset times as well.

The accuracy of the receiver can be between $20 \mathrm{~m}$ to $100 \mathrm{~m}$, which is suitable for most applications. Therefore, some correction techniques been integrated, which can greatly enhance their accuracy to about meters.

GPS is free and available all around the world and that is the main reason it becomes so popular and a rapidly growing applications.

Recently PMUs based on GPS have found wide application in power systems. They compute the positive sequence values of wave-forms as fast as once per cycle. When they collect the information in a central location, they can provide coherent state of the system and make a good start point for most of system monitoring, contingency analysis, protection, and control units.

PMU technology has Potentials for Variety of Practical Applications and they can be divided into online and offline applications: 
PMU online applications use the real-time data, which are collected from phasor measurement units, to control and operate the power system. The online applications can be divided into two categories:

1) Low-speed response applications

In some applications, fast response or quick actions are not going to affect the analysis; they can be summarized as:

\section{1.(a) State estimation:}

Conventional state estimation algorithm used measurements of the lines, the active/reactive power, and the accurate model of the network to estimate voltage angle and voltage magnitude of buses. Prior to synchronized phasor measurement units, the state could not be measured directly. Using phasor measurements, line parameters could be accurately calculated and the dependency on the accurate line model is eliminated. Also, in conventional state estimators, measurements were collected with the update rate of 2-3 times per second, while PMU measurement update rate is 1-60 times per second. This improves the estimation accuracy and speed. In state estimation problems, PMUs not only can measure the state of system directly, but also they can measure both current and voltages synchronously with a high precision and this provides the better ending for this type of problems.

Benefits of Phasor measurement units for state estimation can be summarized as:

- High accuracy and reliability of PMU measurements

- State Measurement instead of state estimation

- Higher rate of update and faster state evaluation, which is good for dynamic state estimation 
- Online topology and parameter identification available through implementation of PMUs in transmission line

1.(b) Power system real-time monitoring and identification:

Phasor measurement units equipped with GPS, has the capability of data synchronization at a common time reference with accuracy within 1-2 microsecond at any global location. This prepares an efficient WAMS, which can provide accurate measurements for the whole power system, even if, it is spread over wide areas. So, the WAMS becomes an attractive application for power system engineers to be used in advance awareness situational techniques, real-time event alerts, accurate event location estimation, and animated event visualization. PMUs can provide much more measurements and information on non-measureable system states, and it can be used as input variables or feedbacks for the controllers. The motivation of this application comes from the fact that the time-synchronized PMU data allows the creation of dynamic snapshots of the system, and those can be used to update the system model, provide an online decision maker, and support to the system operator. In other words, the transmission line and simple machine parameters can be estimated from the PMU data, with high time resolution, and the system event identification can then be provided from the estimated parameters.

The major advantages of PMU for real-time monitoring and identification purposes are as follows:

- High rate of updated data, available through synchrophasor messages 
- Global phasor of the system, available through time stamping with unique time reference provided by the GPS to be used for wide area monitoring systems

- Online parameter and index calculation in real-time with the minimum delay in communication

\section{1.(c) Power system oscillations:}

Protection relays with PMU function have the ability to identify impending instabilities through real-time measurements and to trigger remedial actions in a timely manner to prevent power system outages. Large power demands can result in angle swings in power systems. High penetration of renewable energy sources, such as wind generations, which are characterized by very low inertia and fast response, can results in rotor angle oscillations. It was shown in the literature that the angle of generator bus voltage is very close to the rotor angle. So, generator rotor angle can be estimated through the data acquired from PMUs. This allows early detection of rotor angle oscillations and undesired electromechanical modes. Power system stabilizers (PSS) can be implemented and synchronized with PMU's in order to damp such system oscillations. Main advantages of using the PMUs for a power system oscillation application can be mentioned as:

- Independent but synchronized measurement of frequency and rate of changes of frequency (with the same time reference).

- Active and reactive power flow analysis with fast response for critical applications 
- Fast and simple implementation in microgrids and generation stations

\section{1.(d) Power system operation and control:}

Including GPS-synchronized phasor measurement units to the modern power system allows the use of the new input signal, either local or remote. It can be said that the PMU data within the Phasor Data Concentrators (PDCs) can enhance the controllability of the power system significantly.

One of the main applications of geographically-wide-range synchronized measurements is in improvement of SCADA systems. Additionally, time synchronized measurements are combined with protection, control and monitoring in a multifunction devices called phasor measurement and control unit (PMCU). This provides multiple functions in an economical use of resources because of hardware (Power supply, data acquisition circuits and auxiliary input transformers) and software (microprocessor operating system, diagnostic software, and data processing) costs.

This distinctive of being able to measure the phase angles directly across an interconnected power grid is a key advantage that PMUs have over traditional SCADA. The other key advantage is the much faster sampling rate of PMUs. Other conventional advantages can be summarized as:

- Simple and organized implementation of control methods for distributed generations

- Capability of operation and control within the substation and increasing the range of control zone by integrating broad synchronized measurements through fast communication 
- Enabling the distributed control techniques along with supervised control

- Increase the interoperability of the control system

- Integrating PMCU and enhancing the response for control and operation commands

2) Fast-response applications

Which are power system protection and fault detection.

2.(a) Power system protection and fault detection:

Reference [74] proposed a protection/fault-detection scheme, which is based on comparing positive sequence voltage magnitudes at each bus during fault conditions to detect nearest bus to the fault. In addition to mentioned method. Another scheme can be used for high impedance fault detection in transmission systems [75]. This scheme is depending on the installation of two PMUs at two terminals of the transmission line. Continuous real-time measurements available from both PMUs can be used to calculate the transmission line impedance, admittance and other parameters accurately. Any noticeable change in the transmission line parameters indicates a fault or improper operation.

Incorporating time synchronized measurement in protective relaying and metering systems provides the benefits of synchronization without many additional components.

There are also several other applications for synchrophasors for cases like line current differential protection, distributed bus protection and power swing detection. 


\subsection{Requirements to Achieve secure and Successful Operation}

The development of future smart grid requires a huge amount of research in variety of domains. While many technologies are available for traditional power systems, they frequently are not able to be used in the future systems due to excessive concerns that may prevent the operator from accessing certain functions. Modern power systems have become more vulnerable because of various outages of power grid or communication failure, random disturbances and human errors. Working closer to their critical limit of power systems such as stability, requires more coordinated, faster and more reliable measurements, processing, and control. Therefore, novel approaches needed to be explored for these recent technologies and some will be discussed in this dissertation. In addition the physical system the cyber aspect must also be addressed from security view point on how the grid's cyber system attributes influence the needs for its operational physical function. Testing in a HIL environment allows the verification of the requirements on execution time, as well as mechanical and electrical compatibility of the different parts of the system.

This research focuses on these topics and it requires the followings.

1. Real time approaches which:

- Integrate with smart grid communication protocols

- Enables the safer and more reliable operation of power systems

- Incorporates useful awareness through wide area real-time data

2. Models and metrics for emulation of the smart grid which incorporates:

- The ability to validate the old system, new improvements, expectations and solutions in future 
- Current smart grid models, information, and technologies

- Realistic system along with flexibility for both known and unknown issues

3. Smart grid research testbed that:

- Provides environment to explore operational and real world issues and their impacts on the system

- Supports evaluation of various control, monitoring, security, protection mechanism

- Integrates both cyber and physical system properties

A good designed testbed can benefit four primary users:

- Technology Vendors

- System owners

- Regulatory agencies

- R\&D institutes

For most of R\&D institutions like national laboratories, companies and specially universities, testbeds are crucial to understanding current technology issues and developing next-generation methodologies.

Testbeds also provide a key role in education and training. At the university level, students can learn about current technologies and gain practical insights into the operation of modern power system. Additionally, utilities and independent system operators can leverage them to educate operators about best practices to prevent, identify and respond to real world issues. 


\section{DEVELOPMENT OF SCADA SYSTEM FOR REAL-TIME OPERATION OF POWER SYSTEMS}

\subsection{Introduction}

Supervisory Control and Data Acquisition is a system for gathering and analyzing real-time data. It is used to monitor, control, and supervise in variety of industrial systems, such as water, waste control, oil, gas, and many other plants, as well as energy systems. A SCADA system gathers the information, carries out necessary analysis, and performs required control actions. It also displays the information in a logical, simple,

and systematic form. It facilitates the supervision of systems with detailed subsystems, and usually combines with a Human Machine Interface (HMI). It provides the operators with access to real-time operational data in suitable formats. The inputs are coming from sensors through a communication platform, and the outputs are graphics, trends, reports, and alarms.

Due to the large amount of research on power system issues and implementation of them on the testbed, the need for a new system, which can illustrate the current situation of the power system testbed, is increasing. Different topologies and scenarios may be used for each test setup, and lack of monitoring systems can decrease the value of research outcomes.

In this dissertation, a SCADA system along with a monitoring, in this chapter, in addition to state estimation and bad data detection, in the next chapter, are developed.

A SCADA system usually consists of the following subsystems: 
- A Human-Machine Interface (HMI) is the apparatus, which presents process data to a human operator, and through this, the human operator monitors and controls the process (Can be integrated in LabVIEW).

- A supervisory (computer) system, in order to gathering (acquiring) the data on the process and sending commands (control) to the process (can be integrated in MATLAB, or commercial software analysis package such as Digsilent).

- Remote Terminal Units (RTUs) are connecting to sensors in the process, and converting sensor signals to digital data and then sending digital data to the supervisory system (Can be integrated by CTs, PTs Trough LabVIEW).

- Communication infrastructure is connecting the supervisory system to the RTUs (Can be integrated in LabVIEW infrastructures, serial communication, TCP/IP, UDP, etc.).

There are two basic types of information which are considered in the SCADA:

- Numerical Values:

o System Parameters

o State Variables

- System Architecture

By using the LabVIEW software package, we have the possibility of using the following tools to transfer the data between the LabVIEW located on different computers:

- Shared variable 
- $\mathrm{TCP} / \mathrm{IP}$

- UDP

- Data socket server

The communication system for a SCADA consists of these parts:

- Sensing the real-time values and showing them in each element of interest

- Communicating devices on each element, which transfers the signal to a near computer

- Communicating software on client and server computers, which transfers information from clients to server

The software on a server computer collects the data and merges data into a database and the software on a computer contains the grid topology and sets the values according to the database information and finally, shows them on the monitors.

First, the data should be transferred to them in order to analyze the system using other power system software. To get access to the data or share the data between LabVIEW and other software, two common ways of interconnection are being used:

- Sharing the data through the files: First, the software saves the data in a TXT or XLS file, and then, the other software will read that data from the file.

- Sharing the data through the common memory: In each step of running the software, both types of software have direct access to the memory of the computer, and can read or change that data instantly.

Energy Management systems (EMS) include SCADA, inter utility links, and network applications. They improved gradually and included wide area measurements 
following the 1965 North-East U.S. blackout. The network applications mainly consist of state estimation (S.E), load flow and contingency analysis. Utilities observe and control their portion of the power system by using the SCADA and the real-time data. Power System Measurements in a SCADA System

Real time measurements will be available through the Remote Terminal Units (RTUs) with the latency of about seconds to minutes in SCADA for power system control centers through various communication platforms.

Regulatory and economic requests have forced the utilities to increase the utilization of transmission system with the numbers close to the burdens, and to operate with quick changes and unseen power flow patterns, which will limit the available information for the real-time operation and decision making applications. Higher reliability is now essential due to the extensive cost of blackouts all around the world.

Recommendations from investigations of blackouts showed the need for wide area visibility, awareness, and detection. By using better real-time tools and synchronized data measurement/recorders, they address the problems before their propagation.

Operators are using either offline load flow studies or the classical Weighted Least Square (WLS) state estimation to analyze system security in real-time.

State estimation plays an important role in electrical power systems and prepares the input data for power system applications. It will utilize the captured values to the closest actual state of the power grid based on the redundant measurements and can also estimate the values at the points where there is no measurement. State Estimation Will be discussed in Chapter 3.6 completely. 


\subsection{Information in the SCADA}

Numerical values of system parameters and state variables can be obtained either by direct observation or by estimation. Direct observation is essentially as its name implies actual values that are the result of direct observation. For example a wattmeter provides a direct measurement of the real power flow on a transmission line. Estimation is a more general concept that involves the use of one or several direct observations to compute as estimate of the desired actual values. Such estimation can take many forms. For example, the real power flow on a given line can be estimated from direct measurement made by Watt, VAR, and Volt meters located elsewhere on the transmission system.

The structure type information is the information on the structure of power system. For example, the specification of a transmission network graph and the assumption of a Pi-Model equivalent of each line.

\subsection{Requirements for Integration of a SCADA with Real-Time Capability}

The incorporation and verification of the different methodologies introduced in this dissertation required a SCADA core to be real-time, flexible and accessible for different objectives. Such a SCADA, should interact with control, analysis, simulation, communication, automation, database and archive, or even commercial packages within the subjects related to this dissertation. In addition, to make overall system as hardware in the loop, hardware interfaces should be available and compatible.

The researches involved in this dissertation also need: 
- A comprehensive tool that can build any measurement or control application

- An environment to integrate innovation, discover the benefits and drawbacks of techniques, and finally verification of results

- Compatibility for modular and configurable hardware especially sensors to overcome complex control and communication systems including numerous instruments

- A library of mathematical functions, add-ons, modules and resources in order to approach the solution for any kind of related problems

- A user friendly software development environment, powered to have both consistency and flexibility in extension of modules, as well as a graphical front panel for better integration of the HMI system.

There are variety of options to cover part of the aforesaid requirements especially in the field of power system, such as RT-LAB from OPAL-RT, Matlab Simulink from MathWorks, and RTDS from RTDS Technologies. They have their own advantages and drawbacks; e.g. Matlab Simulink suffers from disadvantages of greatly slowing the simulation for complex hybrid systems and delays causing accumulating errors and phase shifts; RT-LAB and RTDS are simulation only solutions and when it comes to interoperability with actual hardware, they have a lot of limitations.

Due to such situation, it is recommended to move toward a more general and flexible suite, which eliminates the shortcoming of aforesaid well-known packages, but provides us with more compatibility and space for development. 
LabVIEW from National Instrument was selected as the software core to approach the above ambitions. It fluently integrates with NI-DAQs as input/output interface for hardware connectivity and covers a numerous features/ add-ons.

The NI platform has widely been used form educational level up to commercial developments so far.

In this chapter, we introduce our programming efforts to make a SCADA and fulfill the requirements of introduced research topics plus clarify the details of our software core. Our efforts can be classified into two major categories. First, the developments in LabVIEW environment, and on the side, the several standalone software, which can be executed independently (without LabVIEW).

\subsection{Developed Platform in the LabVIEW}

In this dissertation, a lot of Virtual Instruments (VIs) have been developed in a unique project, not only to fulfil the objectives of this dissertation, but also to prepare an environment for collaboration with other related projects.

The developed software platform needs to have instruments and cover subjects, such as SCADA, online monitoring and operation, synchronization, calculation of parameters, communication, control, third party software collaboration, database and archiving, and supplementary special tools for the intentions of this work.

For this purpose, a main project was defined to unify the system and apply a standard and a compatible development by different users. 
Figure 3.1 demonstrates the LabVIEW project with several instruments, which are developed for various applications. Each VI is made of an observable front panel, and a programming backbone, which includes internal structures and sub-structures.

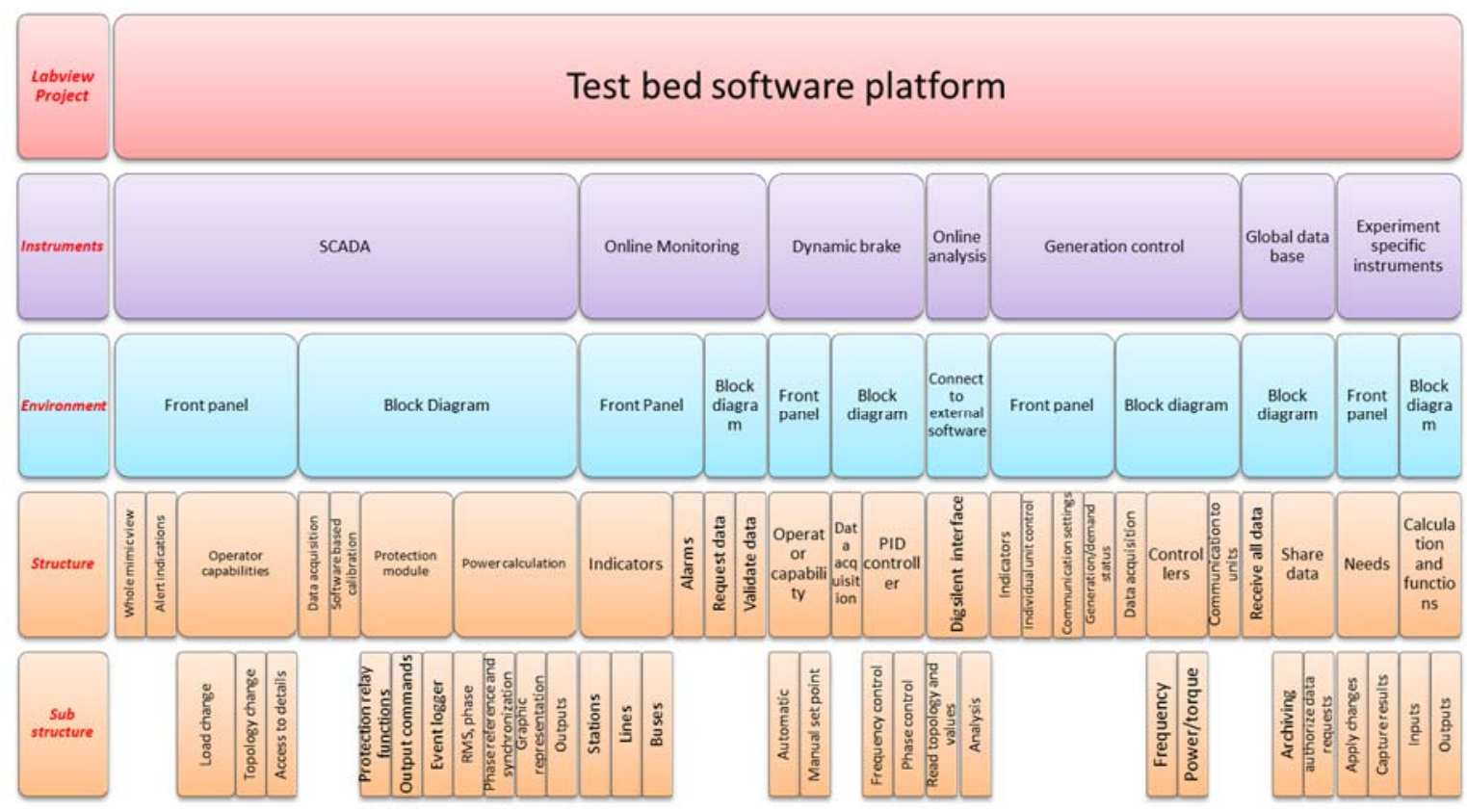

Figure 3.1 LabVIEW structure

Detailed information of VIs, which were developed, will be explained in the following sections.

\subsubsection{Modular Structure of LabVIEW Platform}

Modular programming helps to keep the code optimized, clear, and comprehensible. As can be seen in Figure 3.2, the data acquisitions are made by a separate VI, and SCADA is subscribing to its results; then, the SCADA will make use of sub-VIs and provide the required calculation/process to be exported on its front panel or to be published for other VIs' application. 


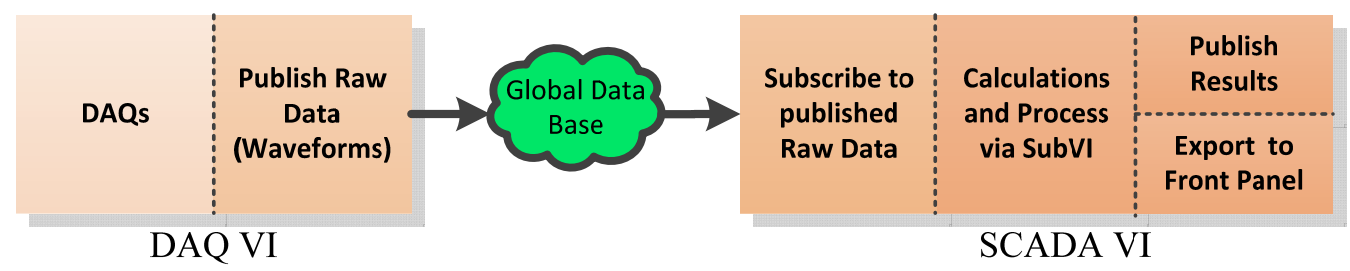

Figure 3.2 Schematic of modular SCADA

\subsubsection{Global Data Acquisition and Database}

Data acquisition is the first step of any control/operation/monitoring system. 


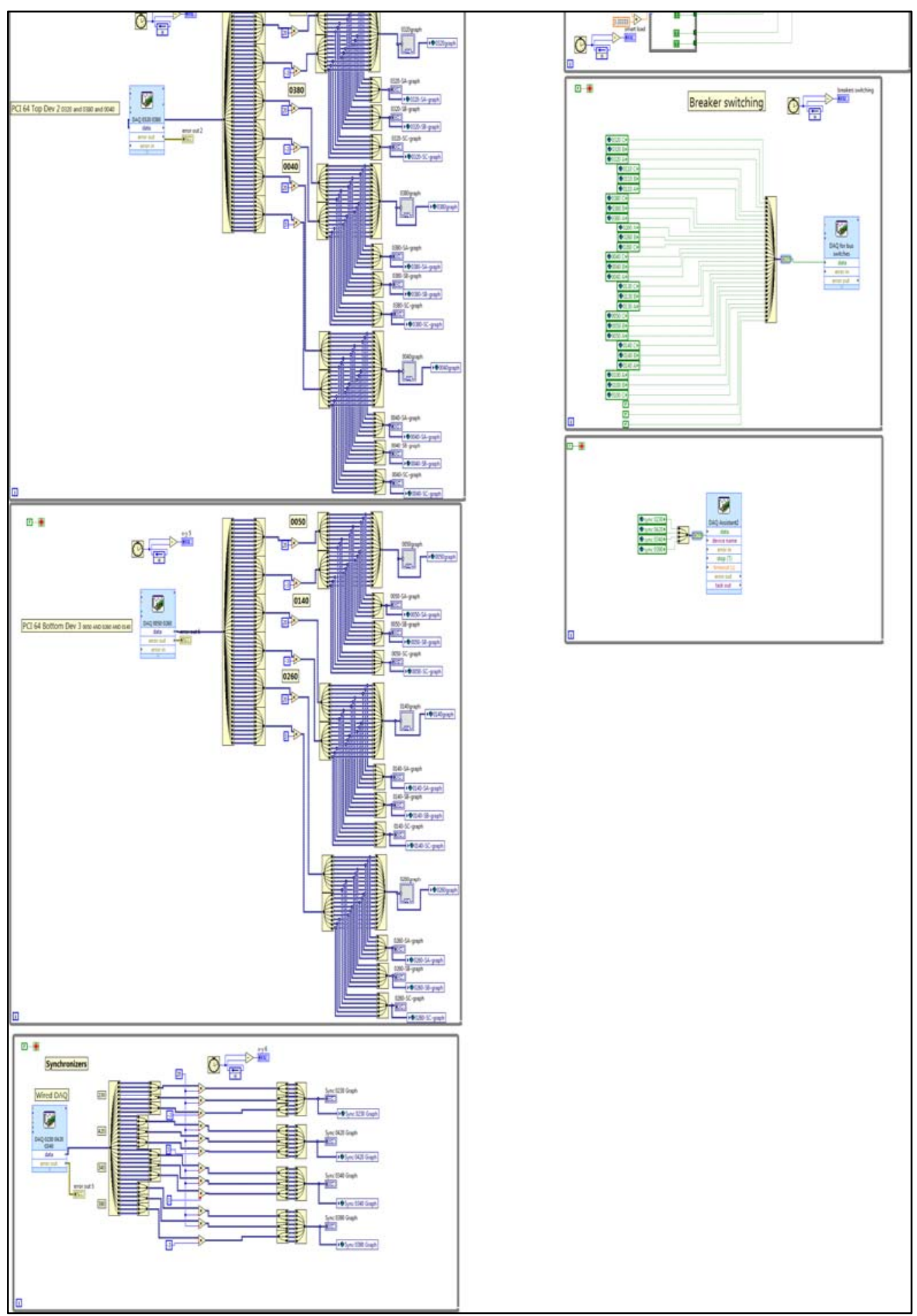

Figure 3.3 Data acquisition VI (Block Diagram)

This procedure will be improved, when combined with a global database, to enhance the data transfer between components of the modular program in the LabVIEW 
environment. Global variables in the LabVIEW environment will be used not only for input data captured through sensors, but also for keeping the provided results by different procedures, e.g. measurement VI or the outgoing commands, and the circuit breakers' status. In other words, it captures the data, publishes them via global variables, subscribes the data from global variables, and applies them to the outgoing components, i.e. the digital output DAQs. In order to have a better performance, each DAQ needs to work individually, and with a particular sampling configuration.

\subsubsection{Modular SCADA}

Figure 3.4 shows some of the modules, which are used in our new SCADA. In this example, 3-phase feeder "0050B", including 3 voltage and 3 current measurements as input, conclude a variety of results, which are packed and published through independent VIs. Here, only the 3-phase active and reactive power calculations are exported to the SCADA front panel.

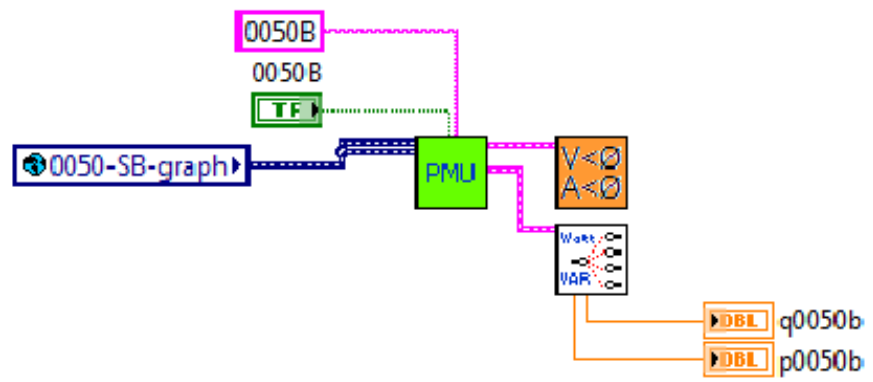

Figure 3.4 An example of modules used in the new SCADA

The front panel of a modular SCADA and a non-modular SCADA can have the same graphic representations, but their block diagram and programming structures are different. It is recommended to use the modular structure for the systems, which are vast in size and have numerous similar components. 
Figure 3.5 represents both of these structures. It is obvious that the similarity of contents helps the modular SCADA to reduce the size and the complexity of connections.

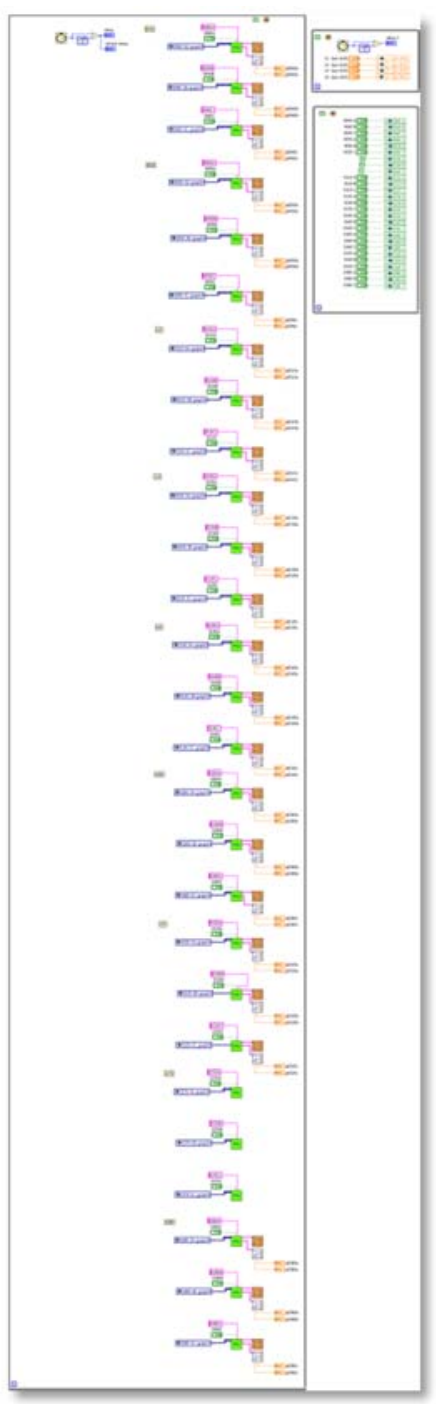

a)

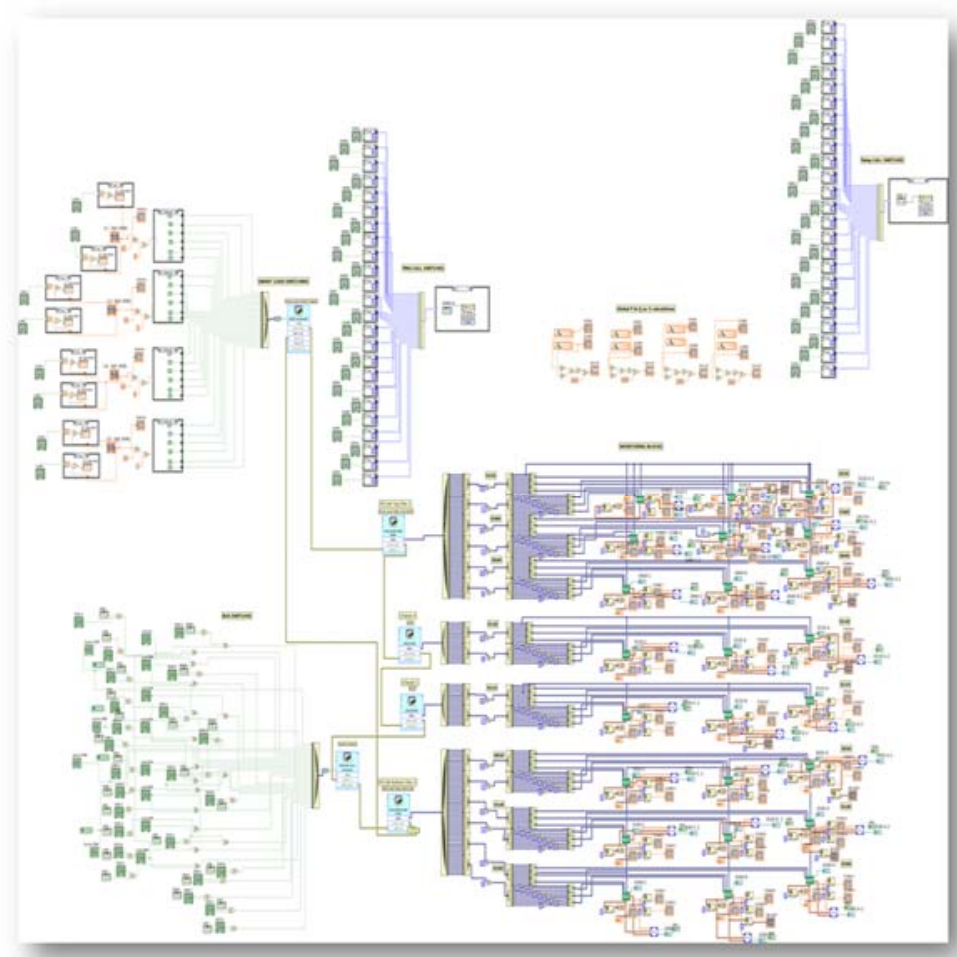

b)

Figure 3.5 Block diagram of: a) modular SCADA b) non-modular SCADA

\subsubsection{Developed Sub-VIs within the SCADA}

The procedure involved in the main project are enormous in terms of input data, calculation processes, and outcomes. By making the software platform modular, it is 
attempted to simplify integrations and operations through several sub-VIs. One of the main advantages of sub-VI employment is effortless duplication for frequent functions.

As an example, Figure 3.6 simply shows a block diagram, from the measurement process to calculation and finally representation, in the LabVIEW front panel. In particular, this only measures $8 \mathrm{AC}$ waveforms from a synchronizer and after verifying the logic, it will send the output power to the controller. Assuming there is a much larger system, we will encounter huge amounts of information and processes. That assumption needs to be performed for real-time application. Therefore, the structure of real-time control and operation software in a LabVIEW environment was designed to be vertical and hierarchical, so it can be modified uniquely and maintain its simplifications.

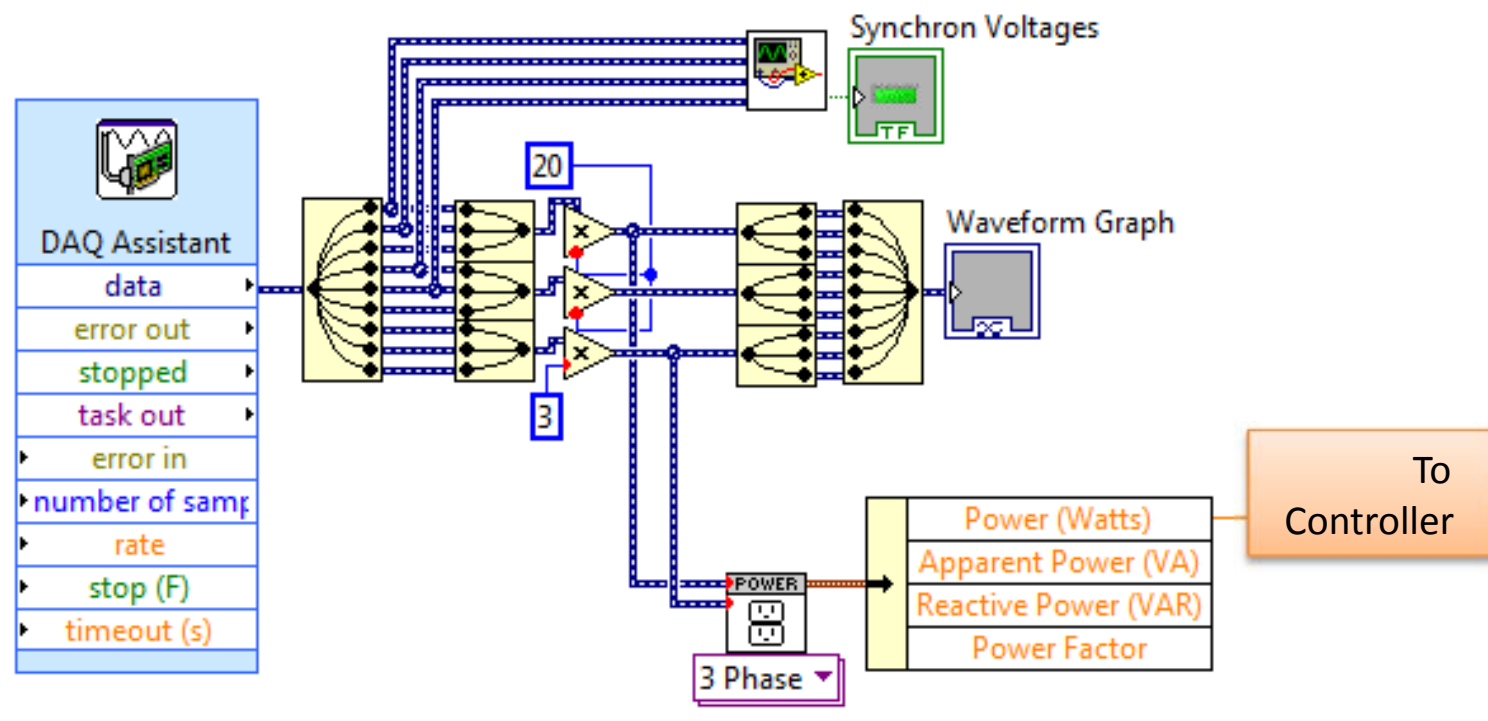

Figure 3.6 Simple block Diagram of measurement, calculation, and presentation of AC waveforms

Some of the major Sub-VIs will be explained here sequentially.

\subsubsection{3-Phase Measurement Sub-VI}

In a 3-phase power system, usually all the raw data (which are Voltage and Current waveforms) are obtained per phase, but their calculated electrical parameter 
results will turn out to be per phase or overall. In systems with a huge number of sensors, these calculations can be performed by one sub-VI structure, but can be expandable to all the raw data independently and simultaneously. Scalability is a key feature of sub-VI which enables it to be used via several applications at the same time. Being at the same time is an internal feature, which provides synchronization of the measurements for applications, such as PMUs, in which it is required to have the same time reference for phase calculations.

Figure 3.7 shows the schematics of this module and Figure 3.9 shows the actual block diagram in the LabVIEW environment.

In order to compare the phasors of voltages and currents, SCADA includes phasor measurements with respect to a phase reference. This can be helpful for integration in protection relay applications, or for archiving to be used for further analysis. The block Diagram of Figure 3.7 can be extended to Figure 3.8 .

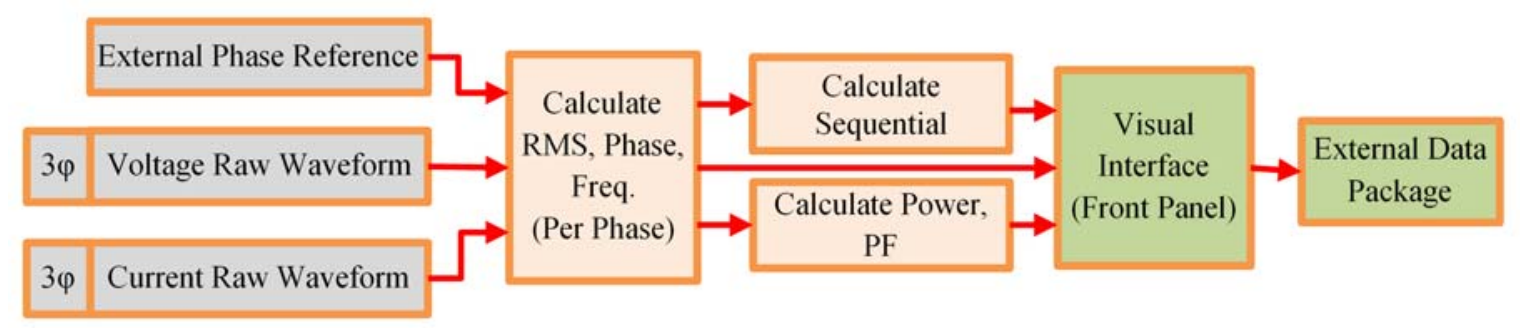

Figure 3.7 Schematics of synchronized measurement sub-VI 


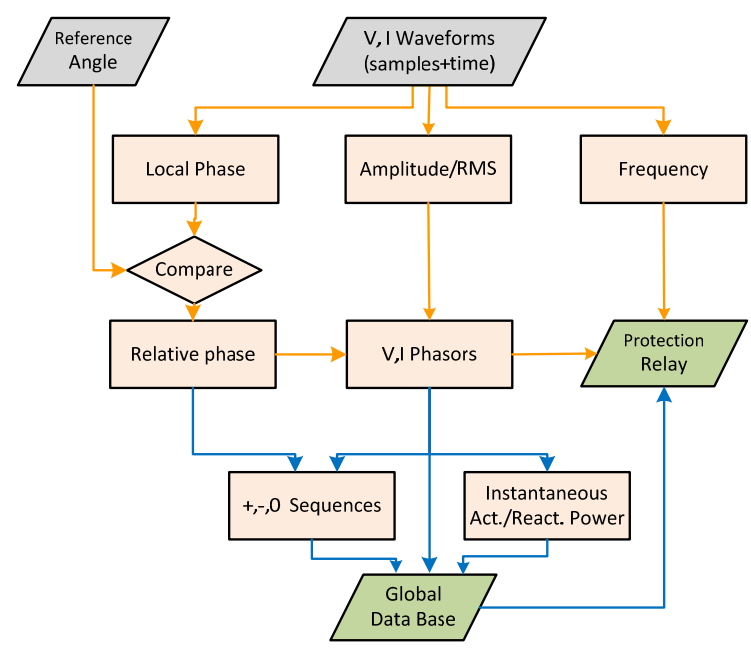

Figure 3.8 Synchronized measurement sub-block

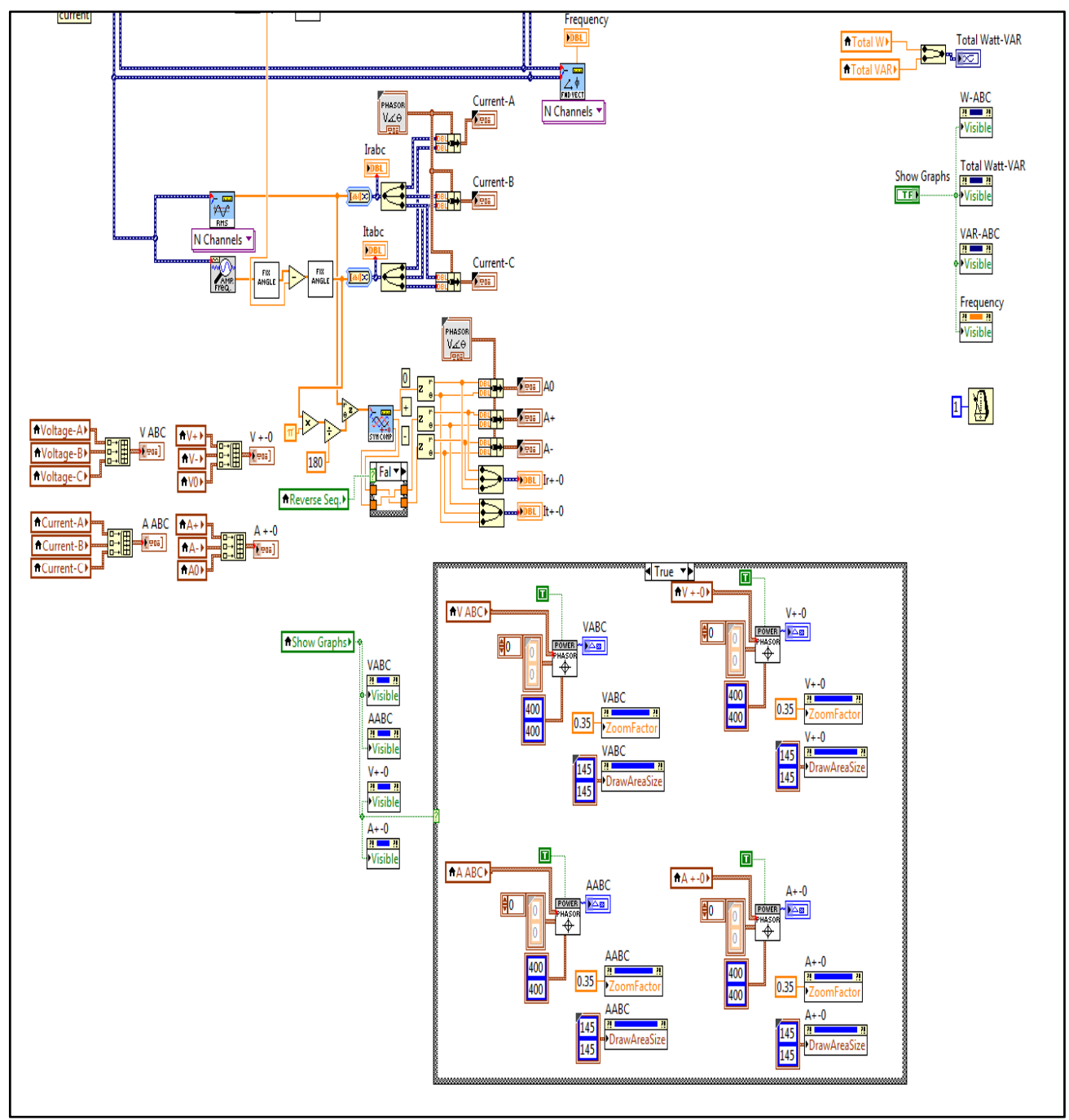

Figure 3.9 Block diagram of 3-phase measurement sub-VI 


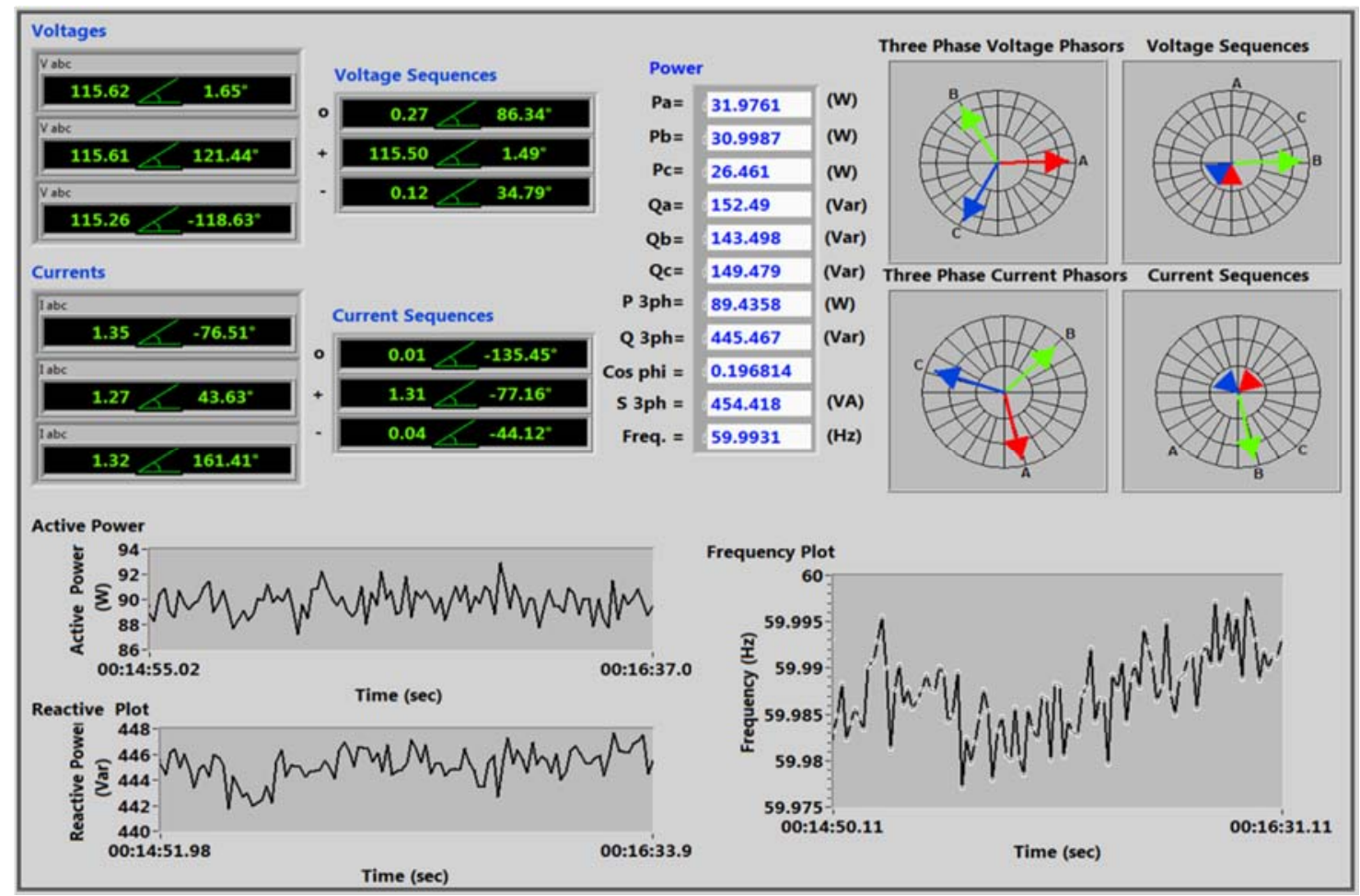

Figure 3.10 Front panel of the preliminary 3-phase measurement VI

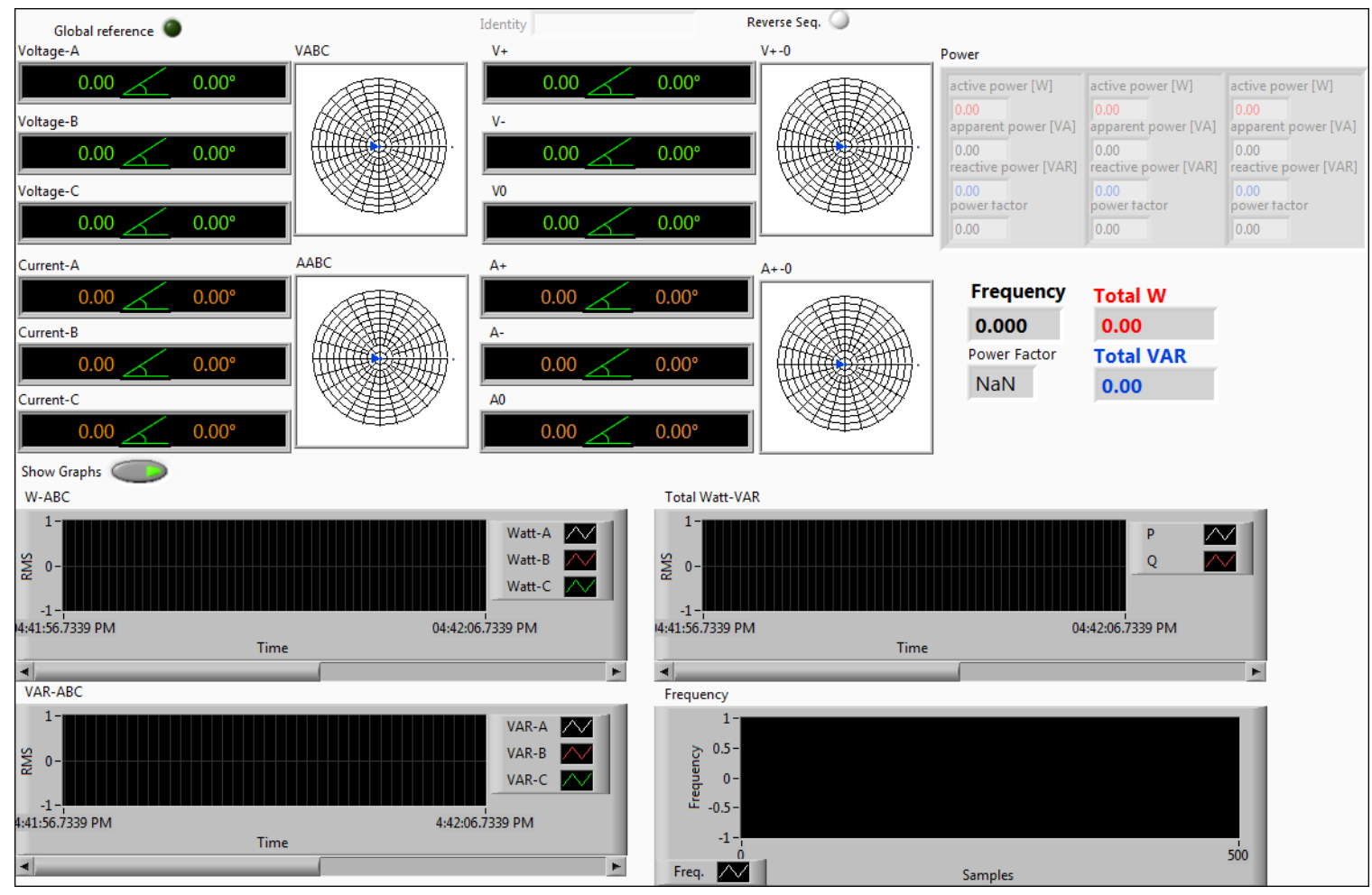

Figure 3.11 Front panel of the latest 3-phase measurement VI 
For every 3-phase feeder, this sub-VI can be used and its results can be exported to the front panel (as in Figure 3.10 and Figure 3.11), or exported to other visualization/calculation overhead VIs (like SCADA, Generation Station Measurement VI, etc.).

\subsubsection{Synchronizer Sub-VI}

The hardware of the developed synchronizer was explained in Chapter 2 and also mentioned that synchronization is a frequent action in power systems and also for our application. Synchronizer sub-VI is in charge of the whole process. It captures the raw waveforms of voltages on both sides of the circuit breaker, performs the calculations, and prepares the logical results of synchronization conditions for any overhead VI.

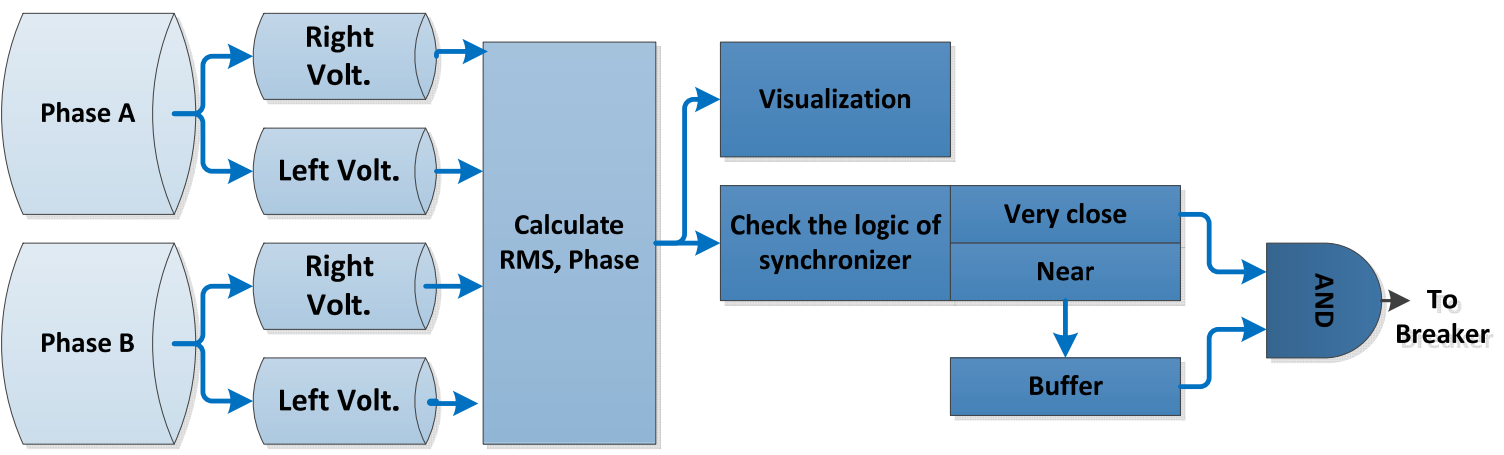

Figure 3.12 Schematic of synchronizer sub-VI

The schematic is available in Figure 3.12 and the corresponding LabVIEW block diagram is presented in Figure 3.13.

The instantaneous status of the synchronizer will be presented in the front panel (Figure 3.14).

The Synchronizer sub-V was improved in terms of optimum programming, as well as front panel view, and finally presented in Figure 3.15. This is also compatible with the modular structure for applications in the testbed or even bigger systems. 


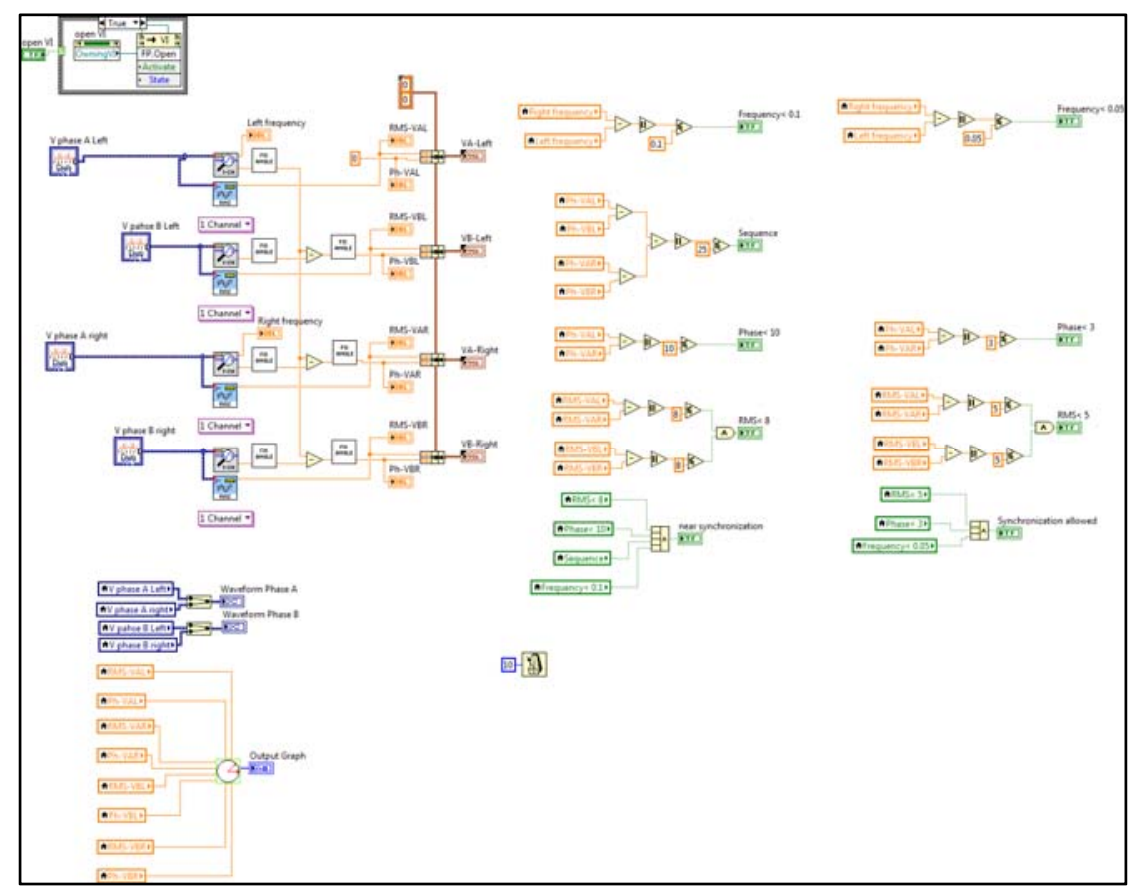

Figure 3.13 Block diagram of synchronizer sub-VI

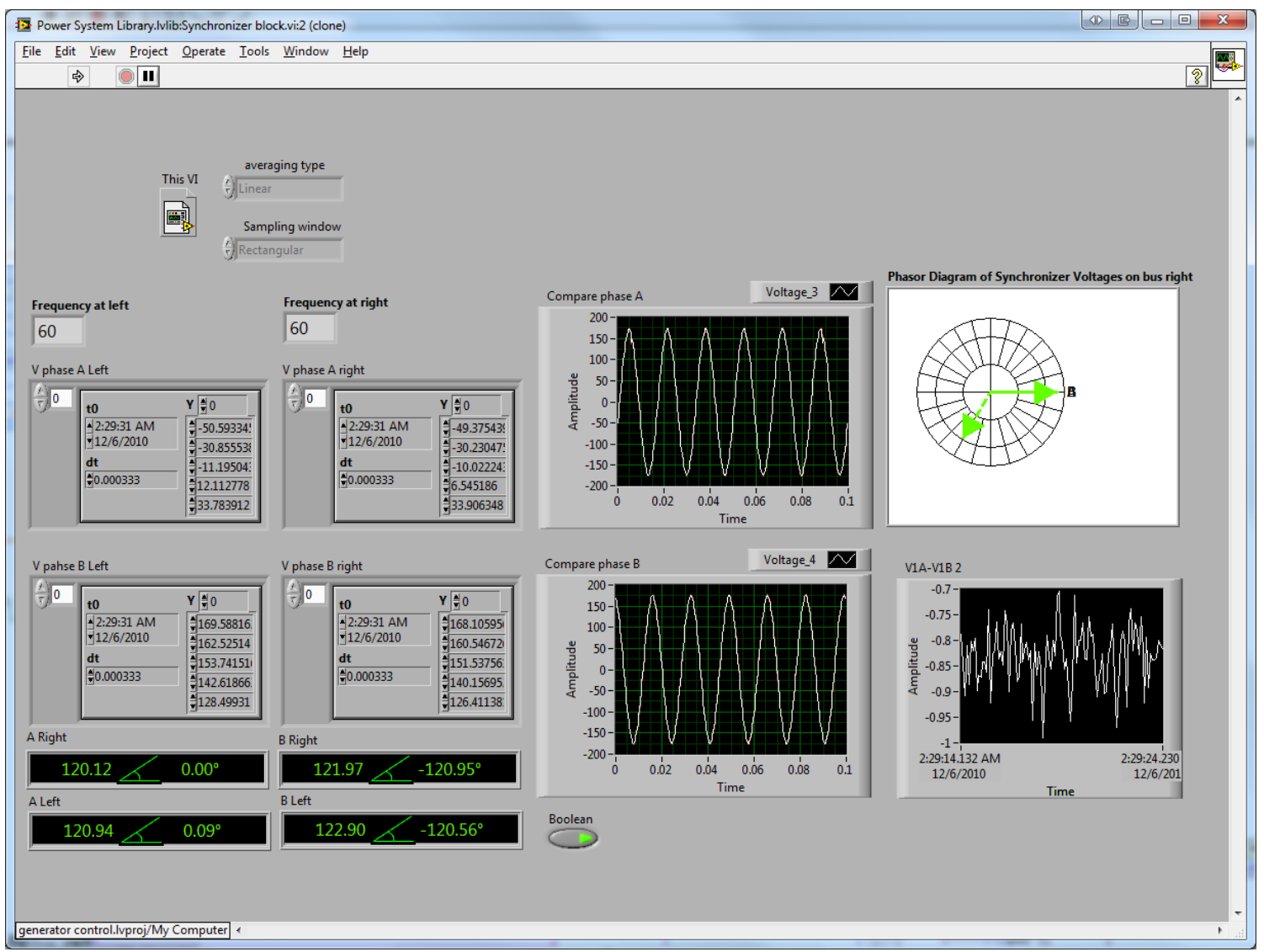

Figure 3.14 Front panel of synchronizer (old version) 
In order to accelerate the visualization, we decided to develop an optimized vector representation sub-VI to be used within the synchronizer sub-VI.

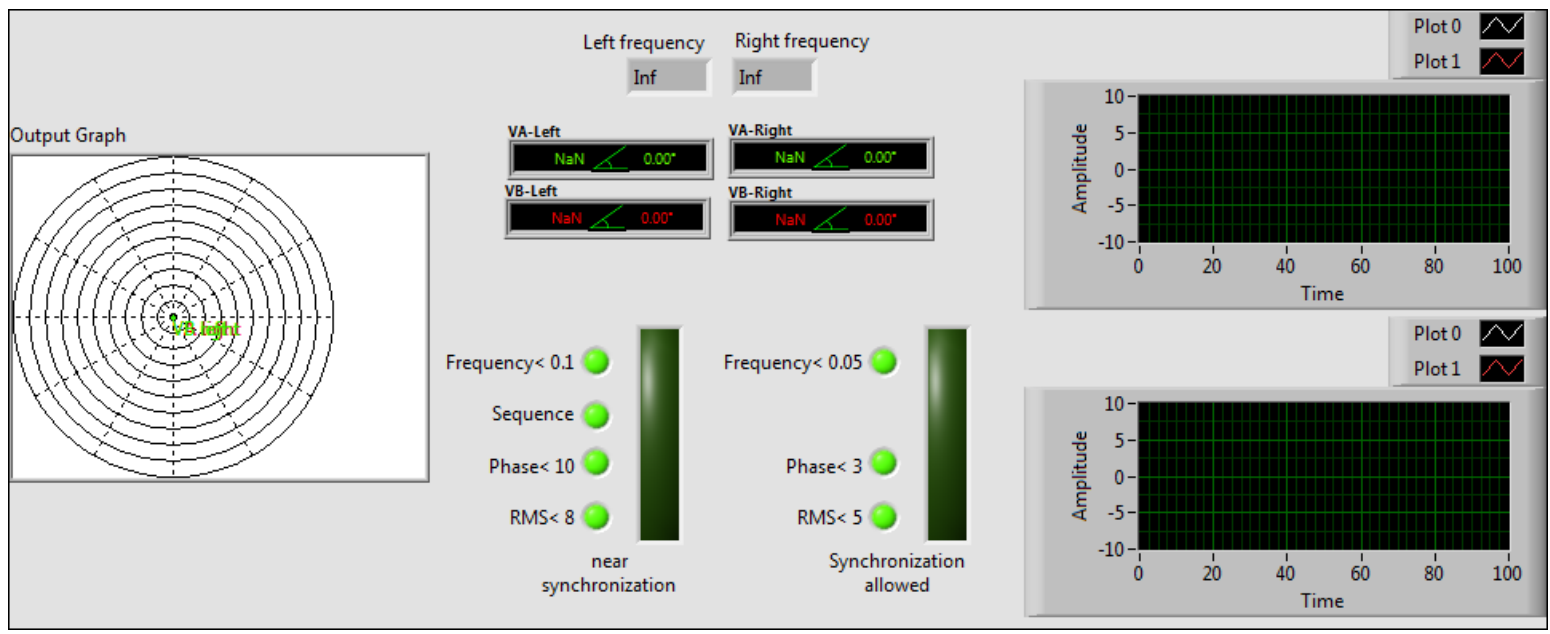

Figure 3.15 Synchronizer sub-VI front panel

\subsubsection{Multi-phase Vector Drawing Graphic Sub-VI}

The multi-phase vector representation of voltages or currents can be provided by this sub-VI, and its front panel and block diagram structure are represented in Figure 3.16 .

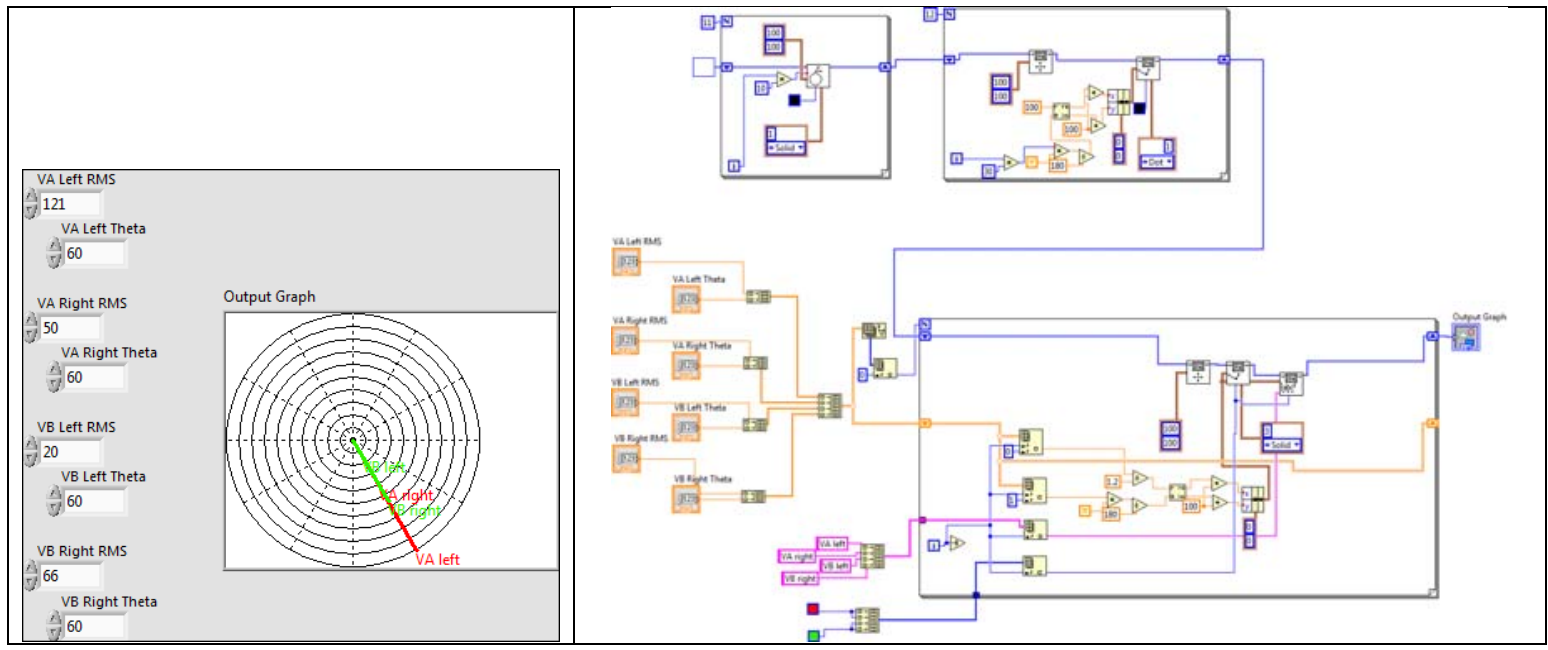

Figure 3.16 Front panel and block diagram of vector drawing sub-VI. 


\subsubsection{Measurement de-multiplexing sub-VI}

Some of the VIs include measurement calculations, and these VIs need assistance to help them organize and optimize their process and results. V-A_VI is de-multiplexing the information of one 3-phase feeder and provides the voltage and current measurements.

Watt-VAR_VI is also a sub-VI, which provides the information of active and reactive power, in addition to power factor and frequency.

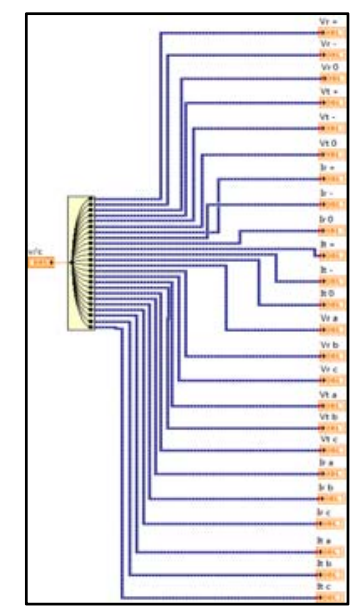

(a)

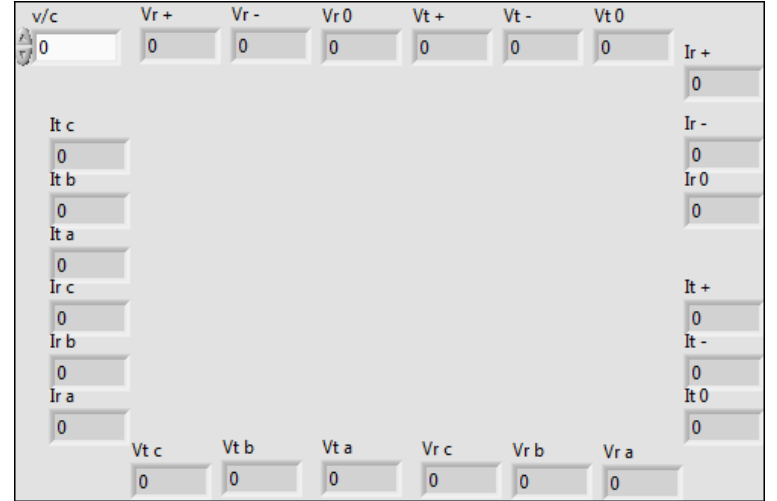

(b)

Figure 3.17 V-A demux sub-VI (a) block diagram (b) front panel

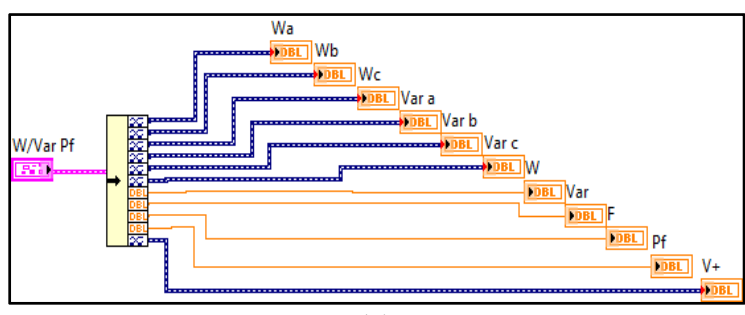

(a)

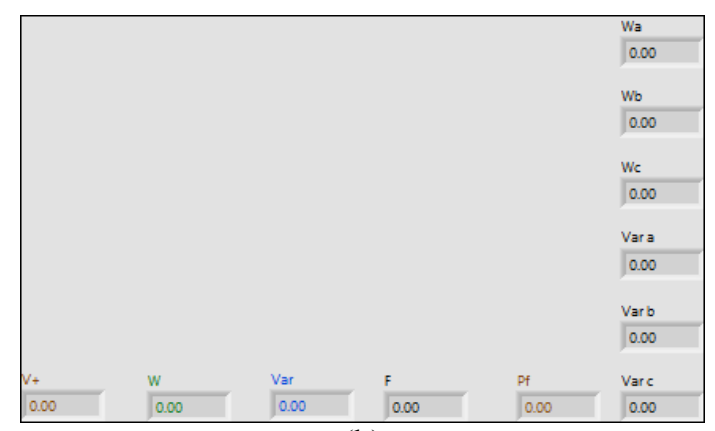

(b)

Figure 3.18 Watt-VAR demux sub-VI (a) block diagram (b) front panel 
There is also another sub-VI, which provides the information of phasors, and can be added to SCADA or other overhead VIs. The block diagram and the front panel of these three sub-VIs are presented in Figure 3.17, Figure 3.18, and Figure 3.19.
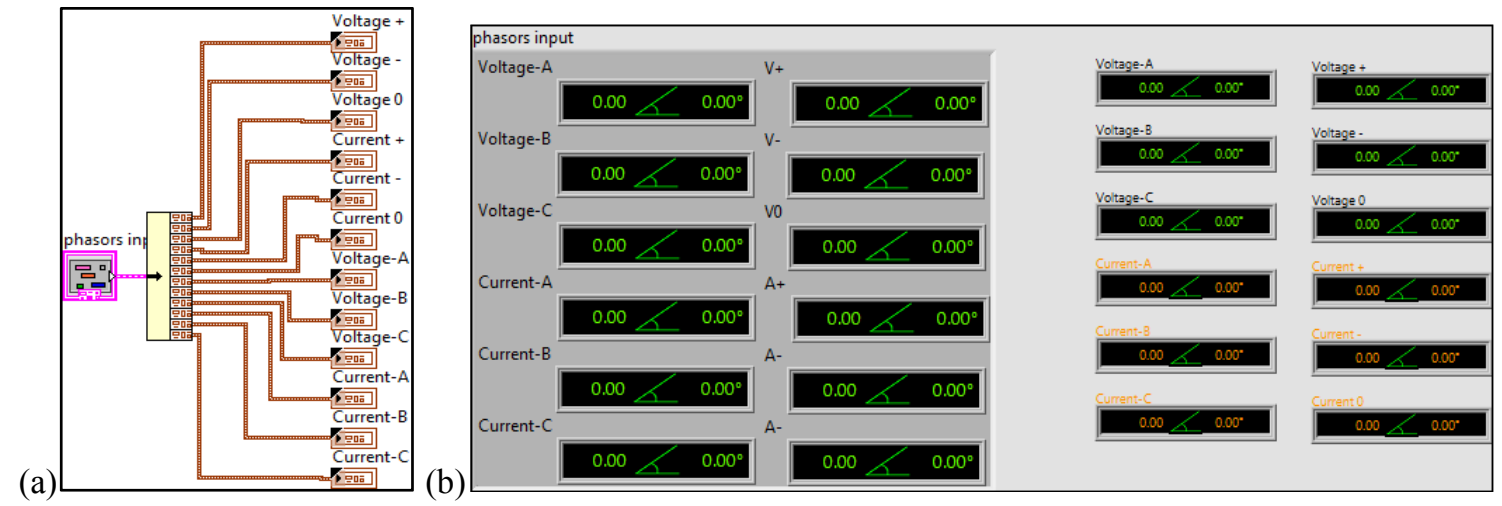

Figure 3.19 Phasor demux sub-VI (a) block diagram (b) front panel

\subsubsection{Generation Station Control VI}

As mentioned in Section 7.2 Generation Stations are equipped with Frequency (inverter) Drives on their prime movers, and in particular Lenze drives are used for that purpose. They have limitations due to their software interface, which is called Techlink. In order to overcome the limitations and also employ the requirements of the research topic, such as multiple generation station communications, control, and monitoring, a new interface needs to have communication with the drives in order to send commands and read their status. It also needs to prepare enough information for the operator to supervise each station in terms of instantaneous electrical parameters. 


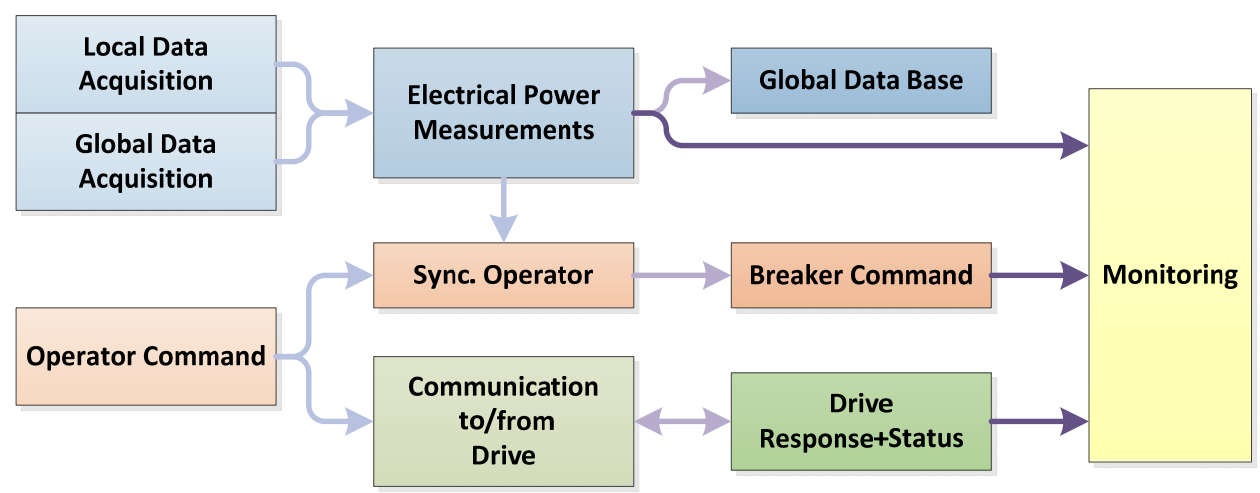

Figure 3.20 Structure of generation stations VI 


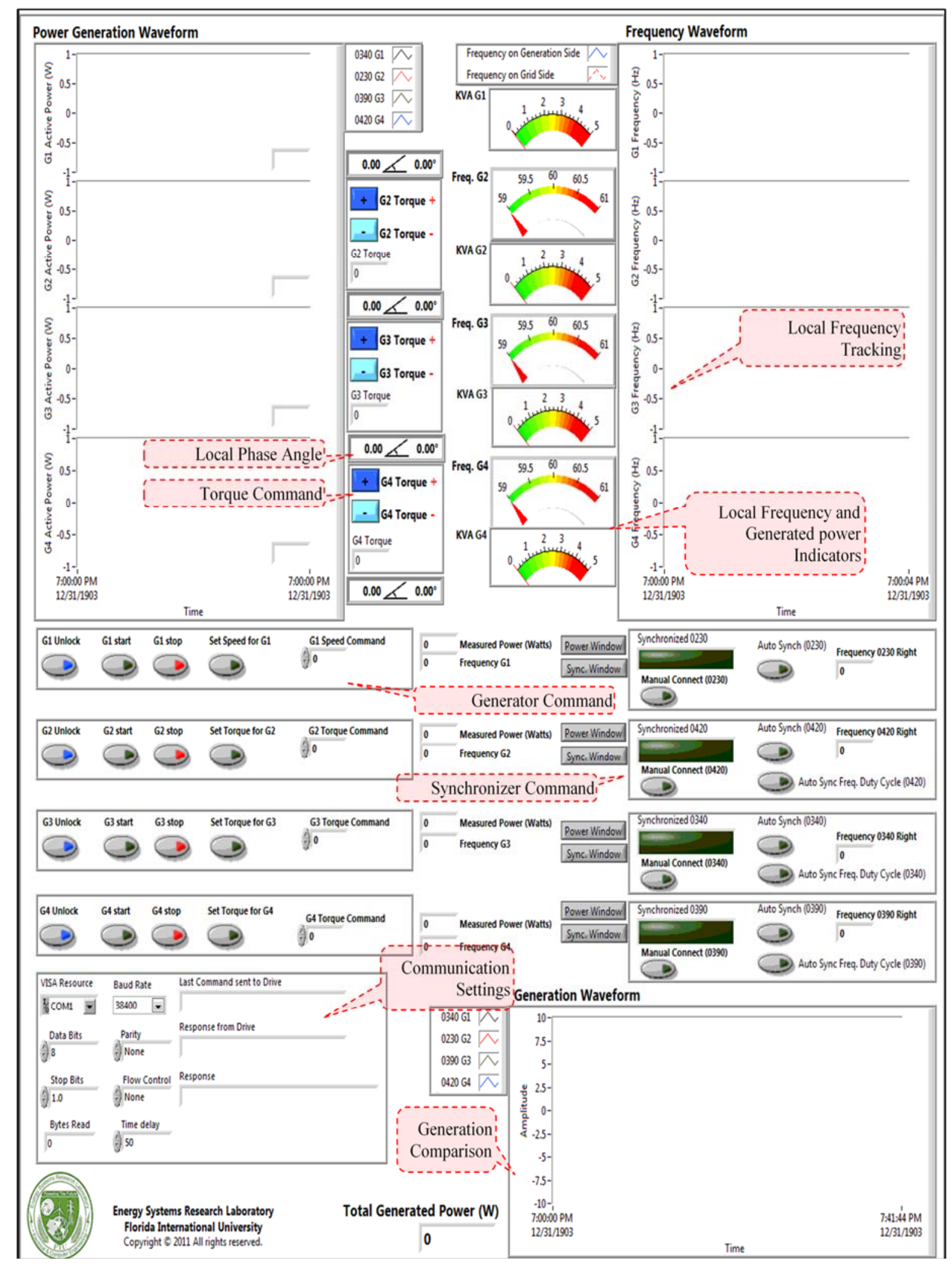

Figure 3.21 Generation station front panel 
Figure 3.20 shows the structure of the developed generation stations control and operation VI. The additional feature of this VI is to facilitate the connection of generation stations to the grid, through developed hardware of synchronizer modules, which are explained in section 7.6.

Based on the requirements, several generation station control VIs have been developed and the latest version is depicted in Figure 3.21.

This VI enables the comparison of total active power generation, in addition to each station.

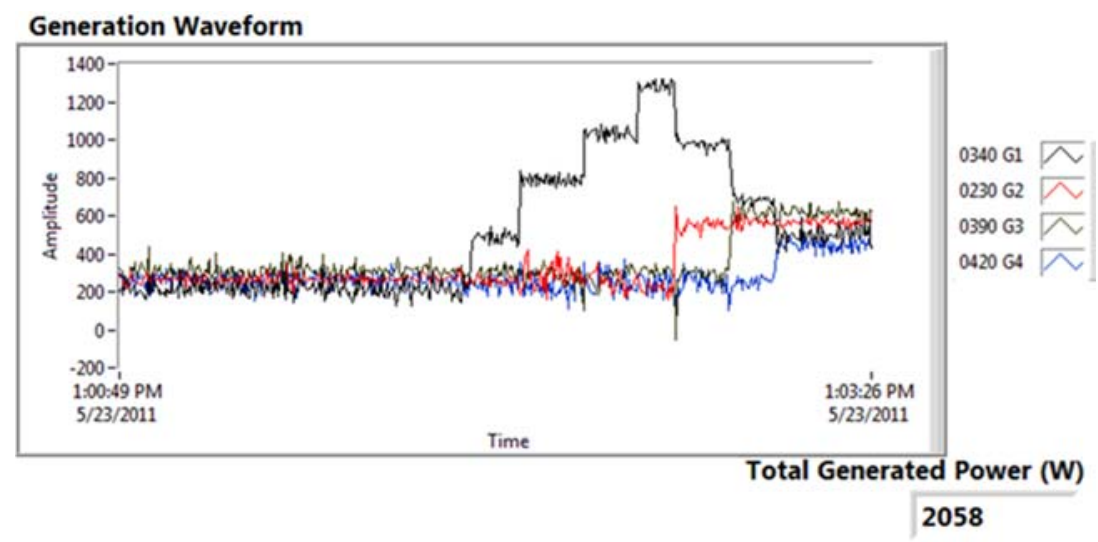

Figure 3.22 Comparison of active power generation in each station

The corresponding block diagram of the generation station control VI is represented in Figure 3.25.

Lenze drives are using Modbus protocol to transfer the information to/from the drives. Modbus is a serial communication protocol, which is widely used in the industry. It is free and popular, with wide range of applications. The Modbus protocol has different structures, and the one which was used is named Modbus RTU and has the structure available in Figure 3.23. 


\begin{tabular}{|c|c|c|c|c|c|c|c|c|}
\hline W & SA & 06 & $\mathrm{RH}$ & RL & $\mathrm{DH}$ & $\mathrm{DL}$ & $\mathrm{CRCH}$ & CRCL \\
\hline RS & SA & 06 & $\mathrm{RH}$ & RL & DH & $\mathrm{DL}$ & $\mathrm{CRCH}$ & $\mathrm{CRCL}$ \\
\hline
\end{tabular}

Figure 3.23 Modbus data frame

Each drive has an address and each frame of information includes several parameters, which are included in Table 3.1.

Cyclic Redundancy Check (CRC) is an error-detecting method, which is usually added to the end of the data frame. It is a reminder of a polynomial division of its contents. If the reminder does not match the expected value, it will be understood that the data frame is corrupted. CRCs are easy to implement in binary systems, and have constant length. The $\mathrm{CRC}$ used here is a $\mathrm{CRC} 16$, which is represented in

Figure 3.24 and it is used in communication with the Lenze drive.

Table 3.1 Modbus Frame Segments

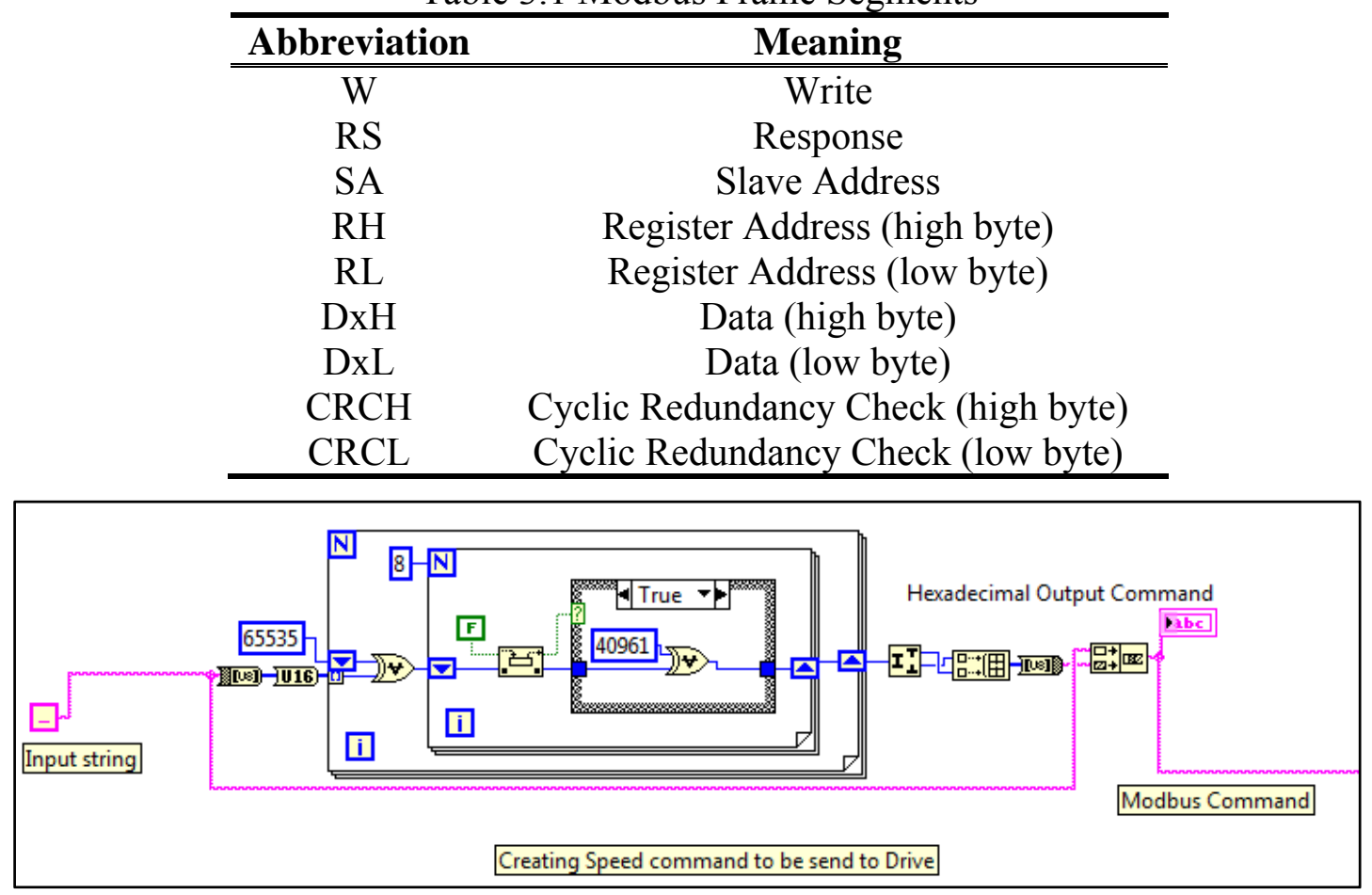

Figure 3.24 Integrated CRC16 in LabVIEW 


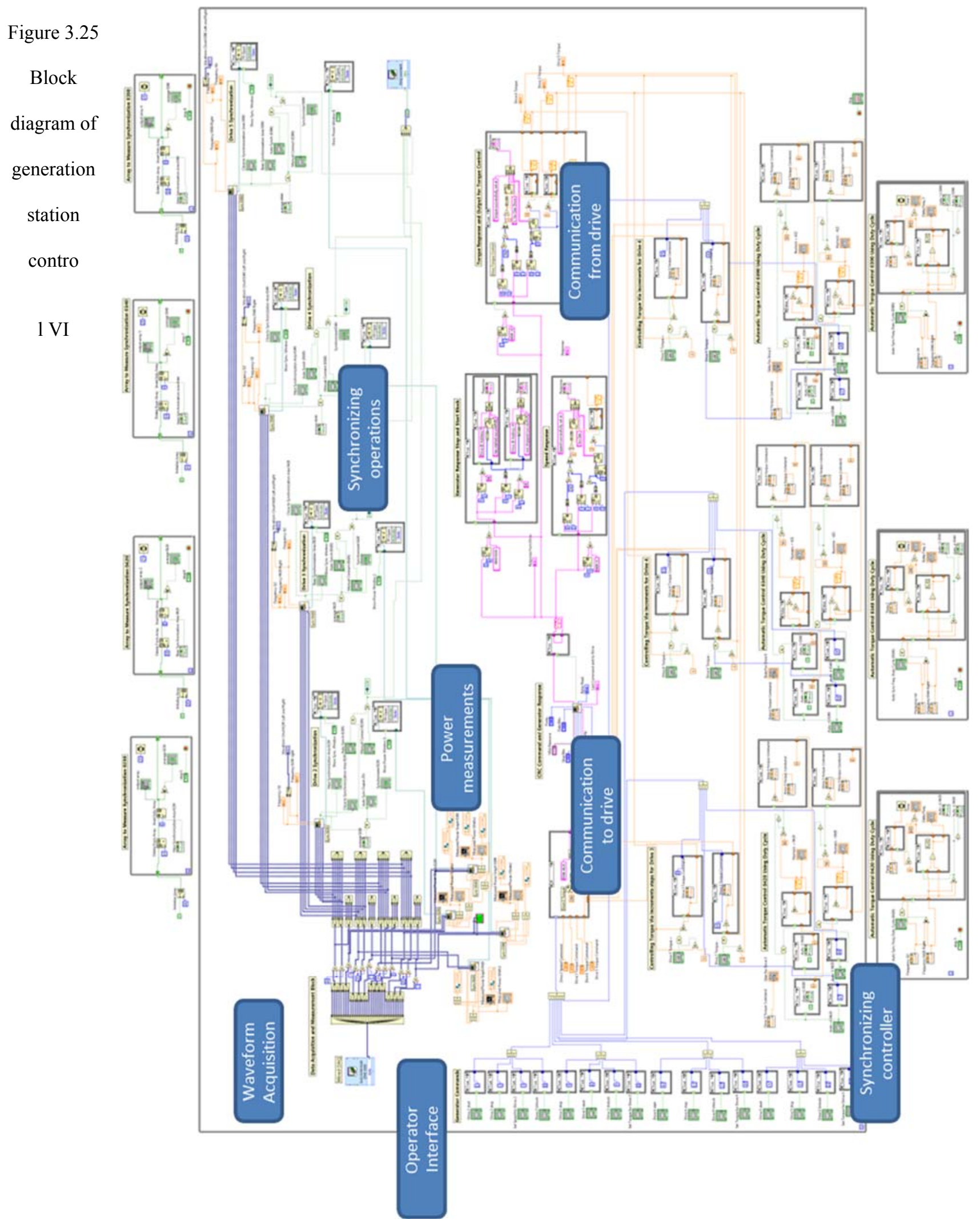




\subsubsection{Developed standalone Modbus Software}

One of the developments beside this work, was a stand-alone serial communication software. It has the capability to attach $\mathrm{CRC16}$, and provides Modbus data frames.

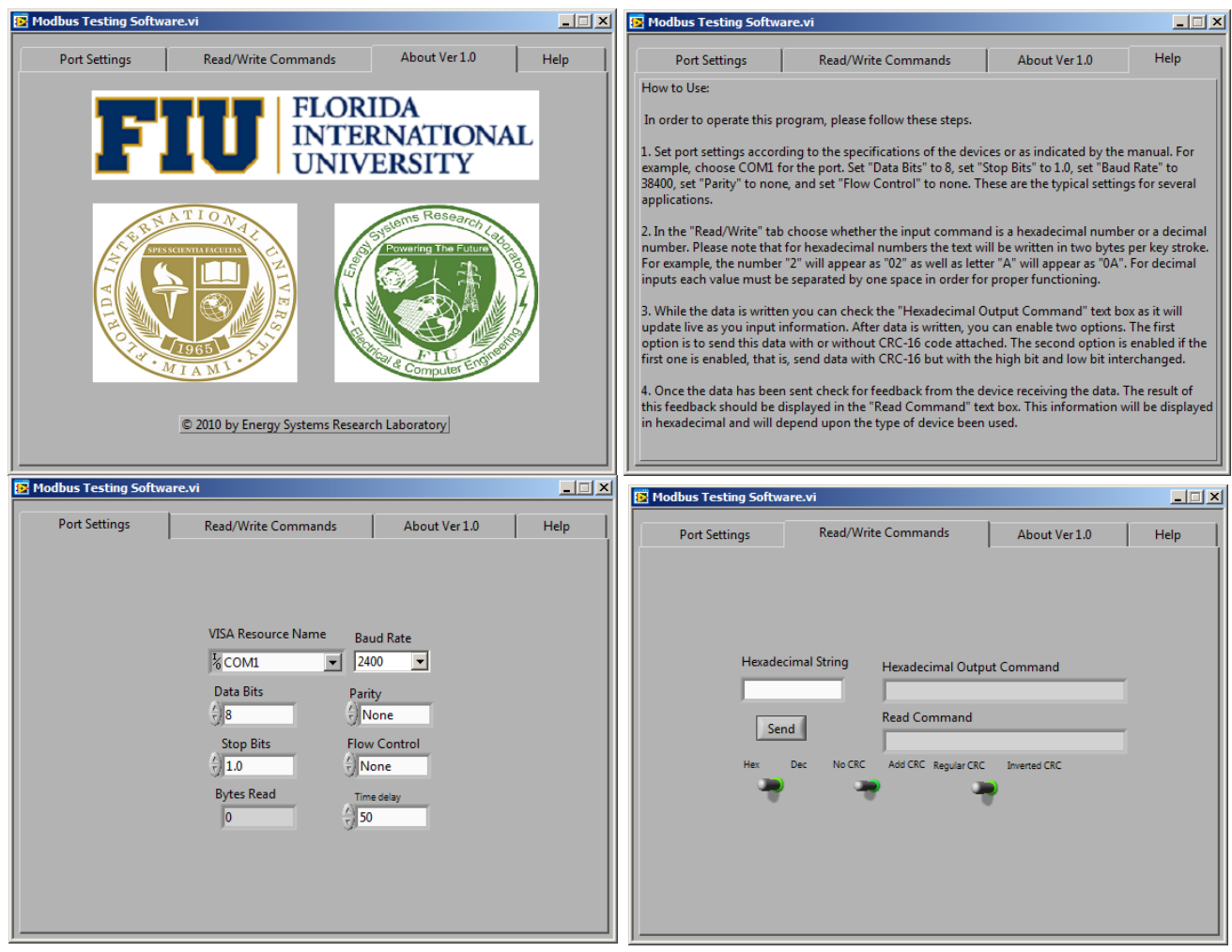

Figure 3.26 Developed stand-alone serial communication software

\subsubsection{SCADA VI}

Like other Virtual Instruments (VIs) in LabVIEW, the SCADA VI consists of two parts:

1. The front panel: which has the human machine interface tools

2. The block diagram: which has the main program, and makes data ready for calculating, getting ready for monitoring, or sending proper commands to any devices, such as DAQ boards. 
Depending on the applications, the SCADA system should be flexible for implementing tools and having scalability features.

In our application, the raw data comes from the voltage and current sensors through DAQs, then the data will be analyzed, and finally, parameters, such as RMS, Phase angle, Frequency, etc, will be calculated. This data can be used by SCADA and other analyzing software packages, such as DIgSILENT Power Factory, and the results can be presented at the SCADA in return as well.

SCADA VI in the testbed requires flexibility, in terms of changes in architecture, and also high enough speed to perform on-demand processes. Several versions of SCADA have been developed for different applications.

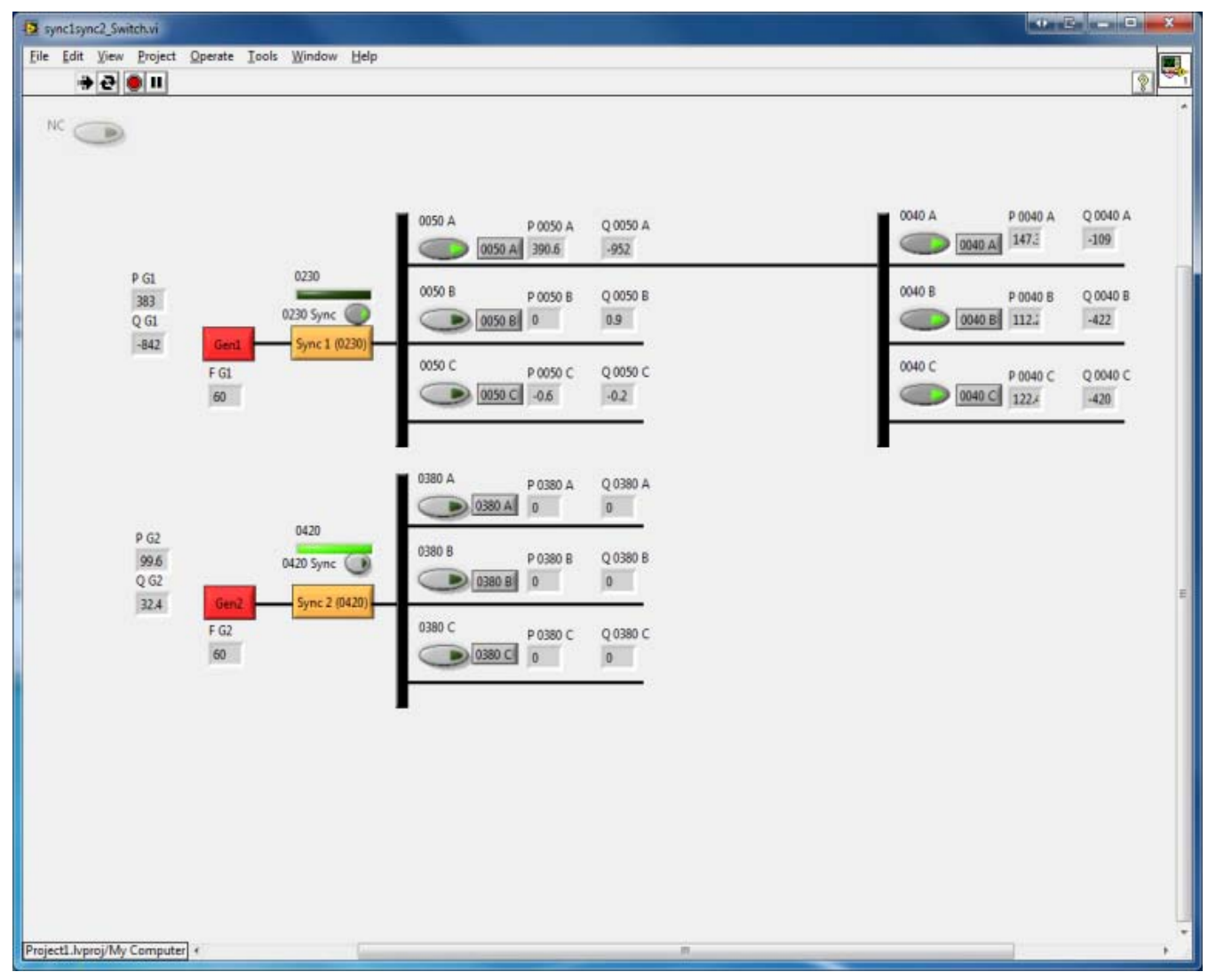

Figure 3.27 Examples of developed SCADA 


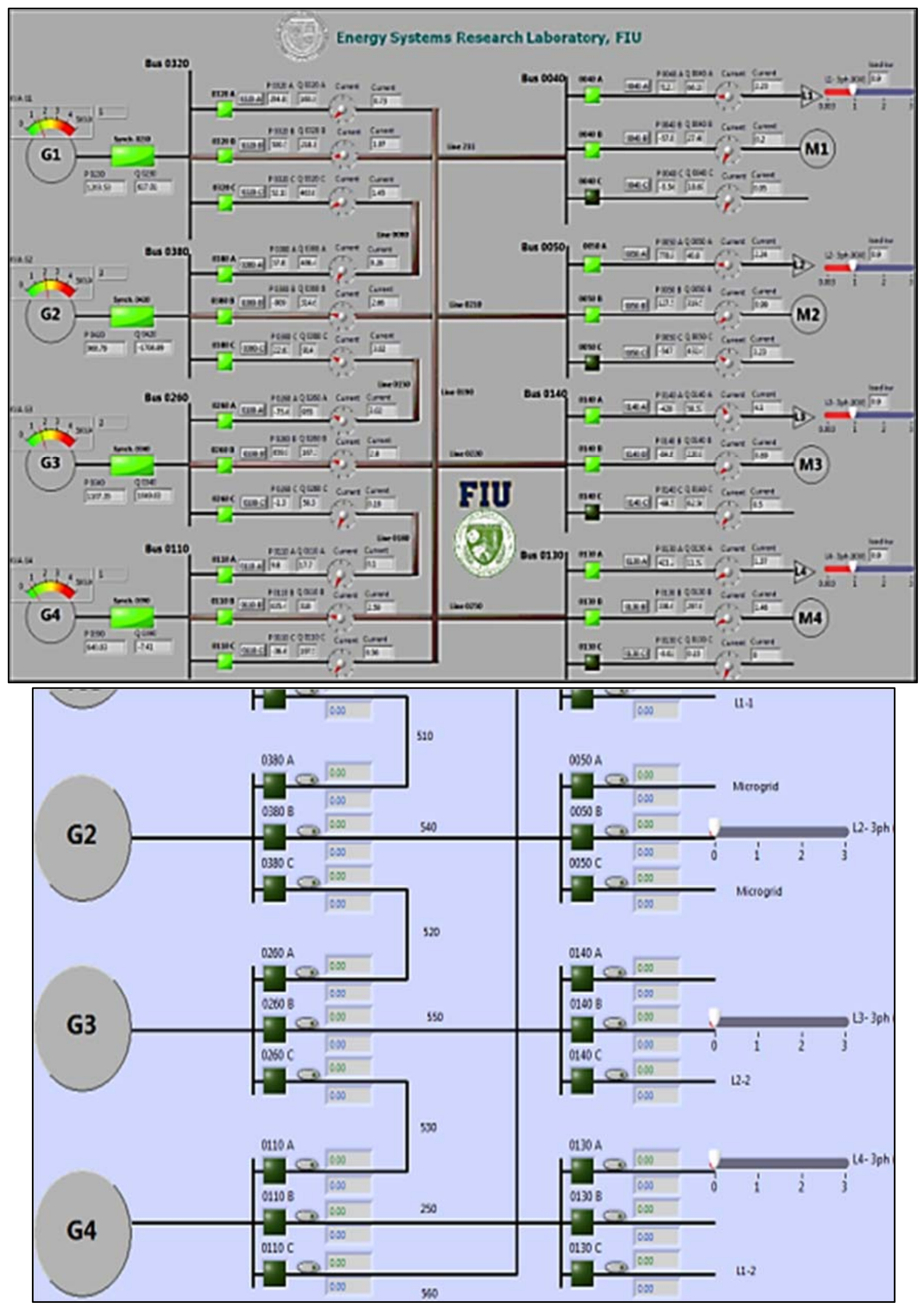

Figure 3.28 Several examples of developed SCADA 


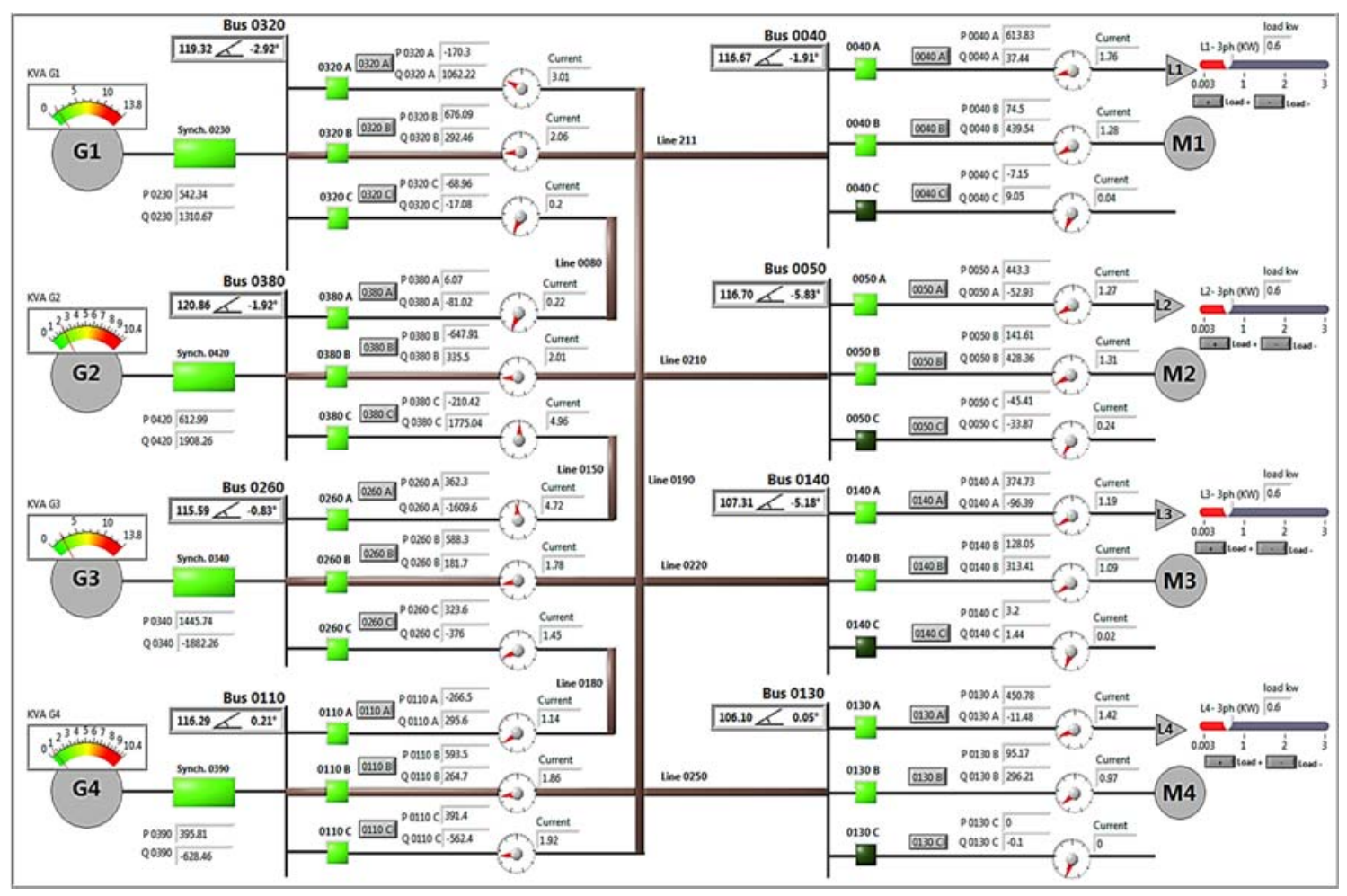

Figure 3.29 Comprehensive front panel of SCADA

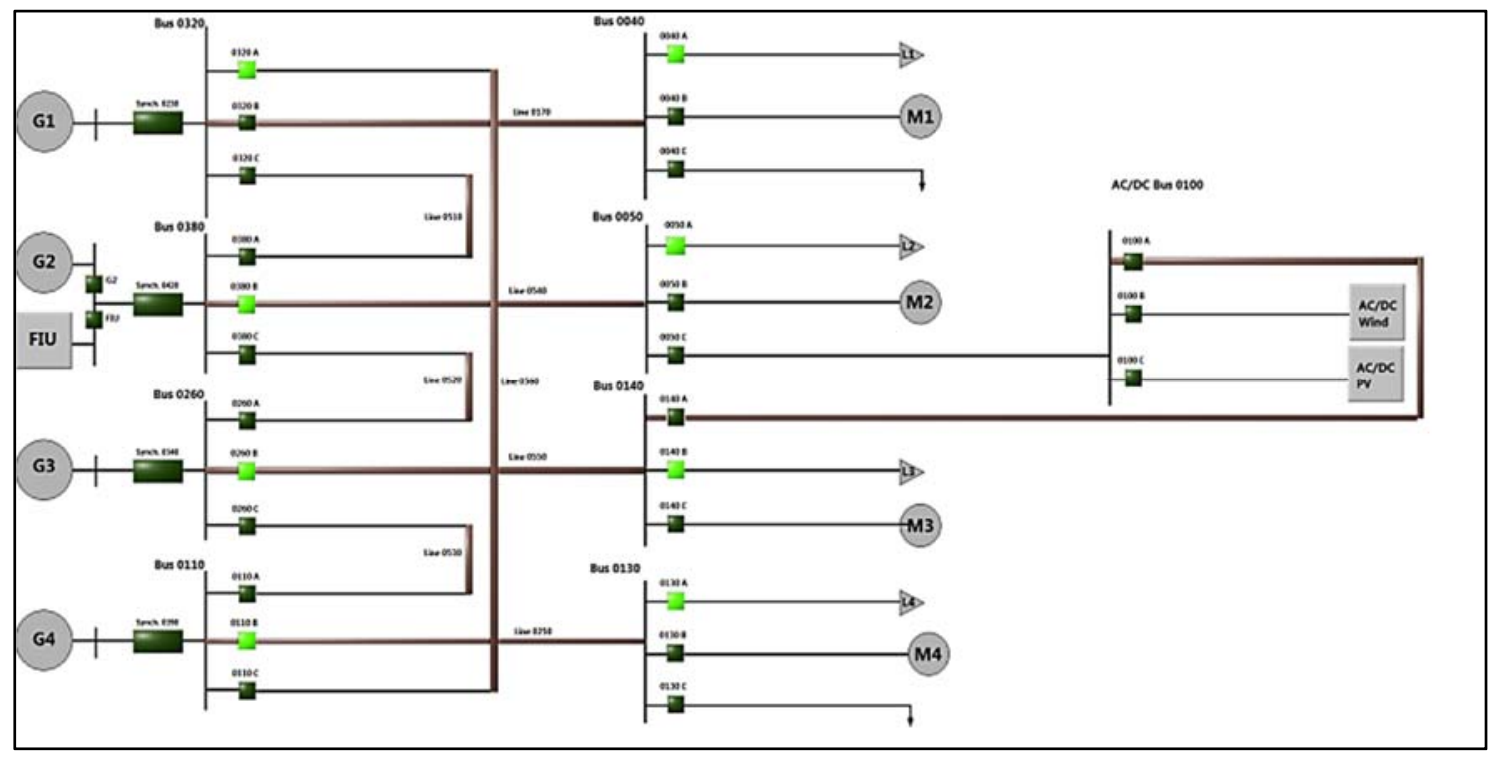

Figure 3.30 A simplified front panel 
Figure 3.27 and Figure 3.28 show a variety of front panels of SCADA. It can contain several types of information for the operator, as depicted in Figure 3.29, or can be a simplified version as in Figure 3.30.

\subsubsection{System Connectivity Online Monitoring VI}

In order to supervise the system, a graphical interface was designed in the LabVIEW environment and it is presented in Figure 3.31. It can include the information, such as bus-voltages, line-currents, Breakers' status, and loads' status, based on the needs/application requirements, either by real-time or offline data.

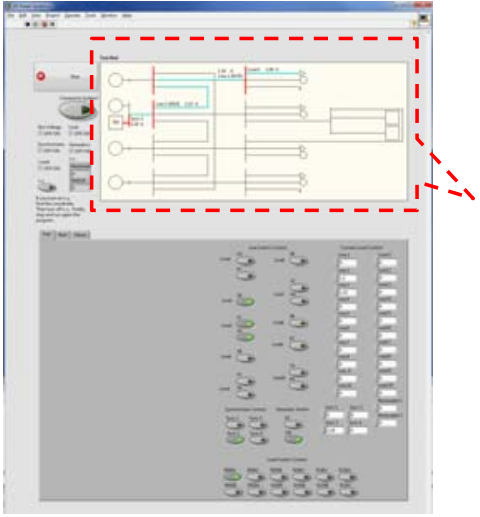

(a)

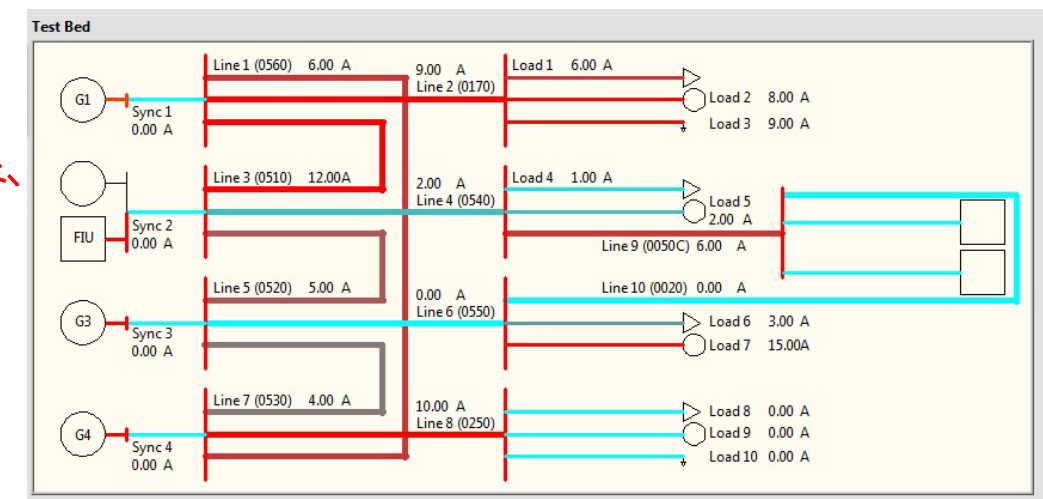

(b)

Figure 3.31 Online monitoring/presentation of SCADA

\subsection{Measurements and Data Acquisition}

The testbed is a real-time platform, which includes hardware along with software features, which empower it to perform optimum operation, to satisfy the goals in the subject of study, and to integrate the scenario needed for the particular research, etc. This is not possible, unless a real-time data acquisition system provides the measurement samples for the analysis. 
Based on parameters like precision, variety, capability and ease of incorporation, the National Instrument Data Acquisition (NI DAQ) was selected to be used for the main measurement platform. NI DAQ is not only a data acquisition device, but also increases the productivity and performance, and that is why NI DAQ is one of the leaders in the field of PC-based data acquisition systems. It has some features, such as simple programming interface, analog/digital I/O, counters, and hundreds of multifunction DAQ hardware modules. Figure 3.32 demonstrate a variety of DAQ types, which have been integrated in the testbed. These can be chosen based on their performance and needs in a particular case study. As an example, some DAQs have 64 analog input channels, which are recommended for SCADA applications, and some have simultaneous sampling which are recommended for synchronized measurement application. Some others have the digital output feature, which is recommended for applying the switching commands, and some have the PWM feature, which is recommended for power electronic applications.

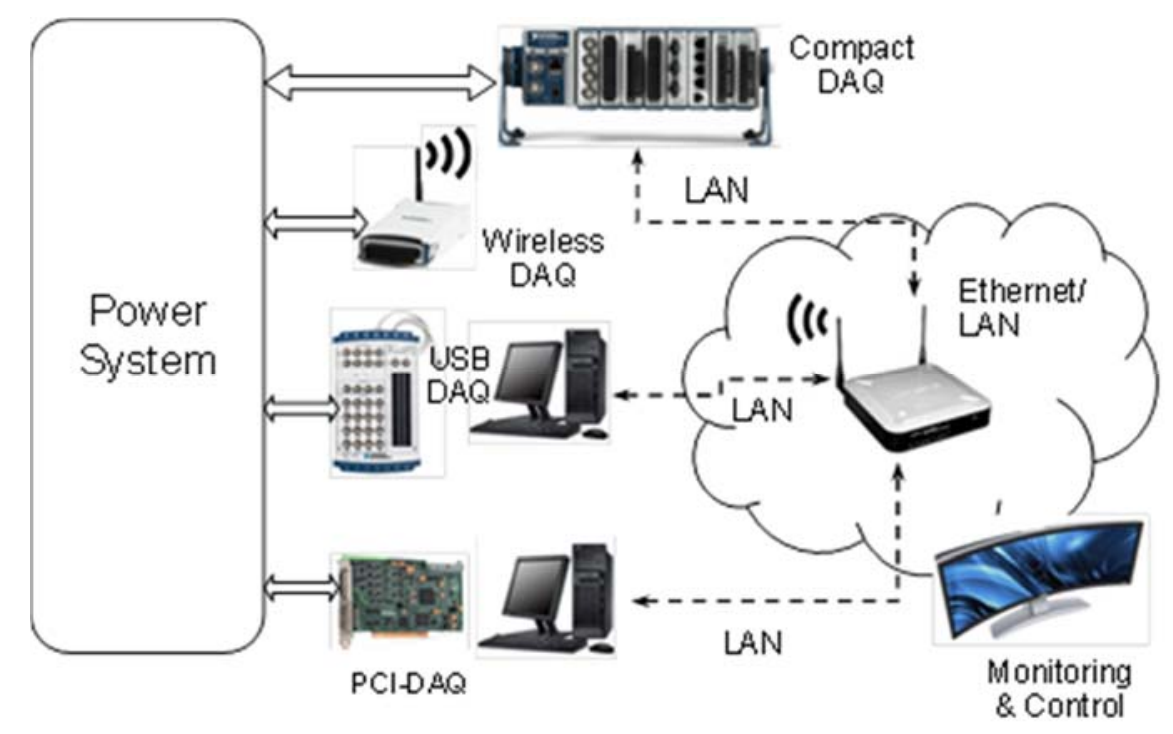

Figure 3.32 DAQs and measurement in testbed 
Figure 3.32 also shows different communication methods, which are integrated for DAQs, in which some of them directly connected to the PCs though USB or PCI terminals, and some are Ethernet/Wi-Fi capable.

\subsection{Calibration and Linearization of Measurements System}

In the SCADA system, the accuracy in the measurement of quantities like voltage, current with their phases is the main criteria. As it can be seen in Figure 3.7 the data flow in power system is initiated from sensor readings. Any considerable error would affect the control of complete system which would definitely change the final conclusions. These errors can be due to different reasons like, improper calibration of the measuring devices, faulty operation of the measuring devices or any problem in the measuring device or measuring technique.

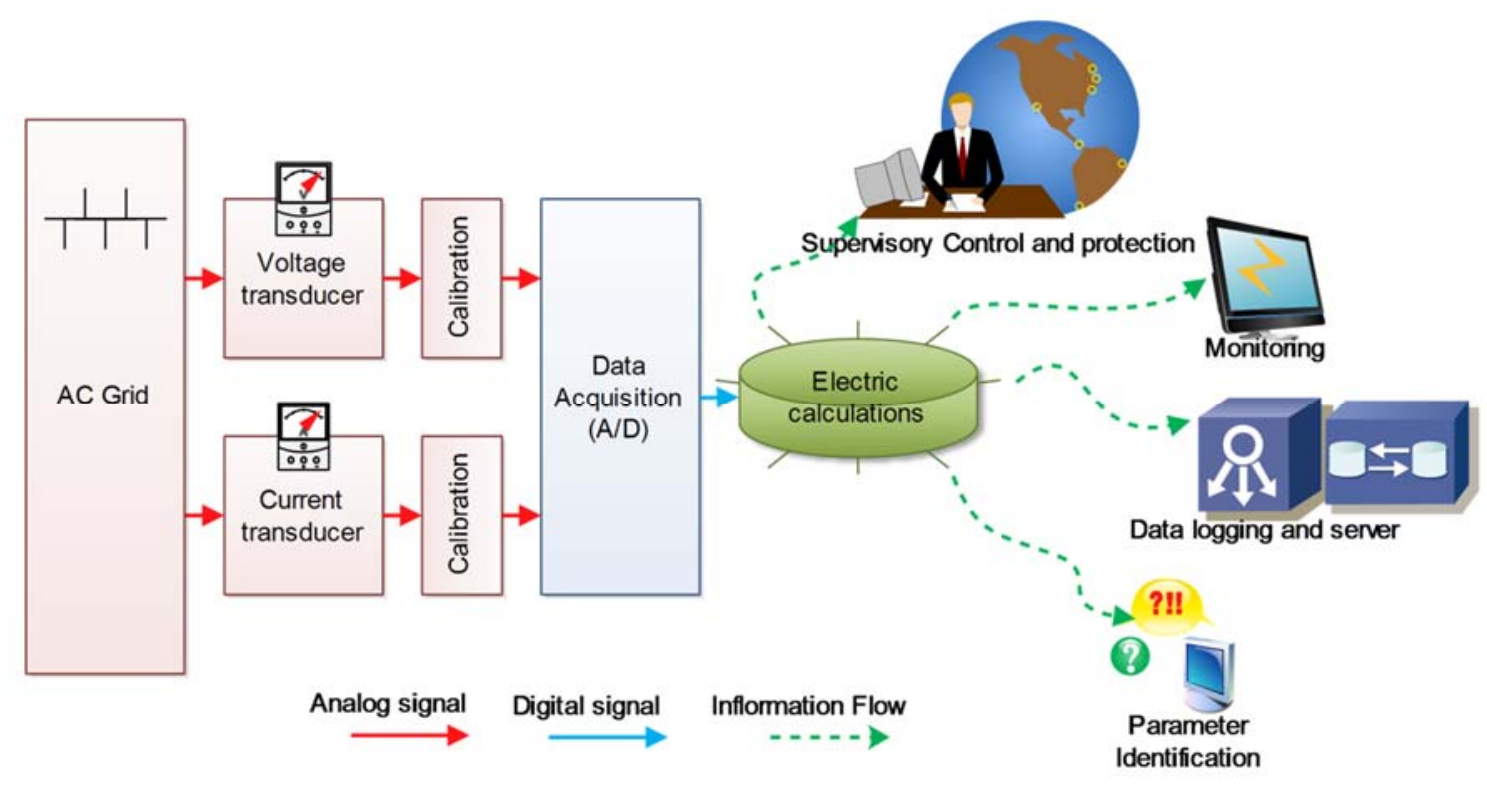

Figure 3.33 Information flow through analog and digital signals in a power system 
As an example, if the line parameters are to be calculated in a smart grid, we need to know the sending end and receiving end voltages and currents. The line parameters should be constant whatever maybe the current and voltage on the sending and receiving end. But it will not be the case when the current transformers (CTs) and the potential transformers (PTs) have faulty operation or even they do not have proper calibration. By giving different voltages to the line it will give different values of line parameters, which should not be the case. This is the non-linear behavior of the CTs and PTs, this affect would be more pronounced at lower voltages. Here in this section, the optimization technique for the CTs is explained and implemented.

In order to compensate the nonlinearity of PTs and CTs, First, it is required to have readings in different voltages and currents. Then, we find the inverse polynomial function, which can map the false measurements (because of nonlinearity of transducers) to real values. In order to make a data base of readings, for PTs and CTs, a calibration system needs to be developed and it is depicted in Figure 3.8 and Figure 3.9.

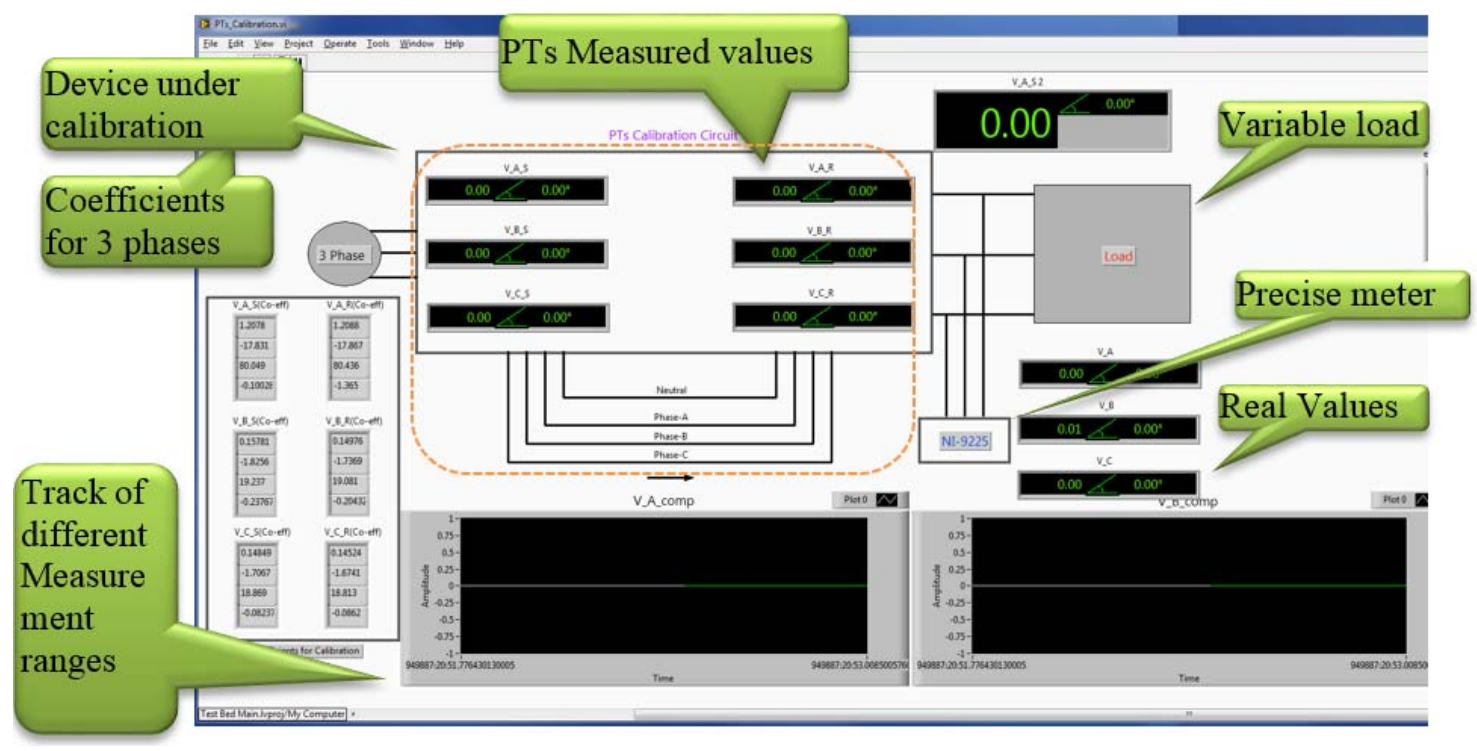

Figure 3.34 Developed PT calibration module 


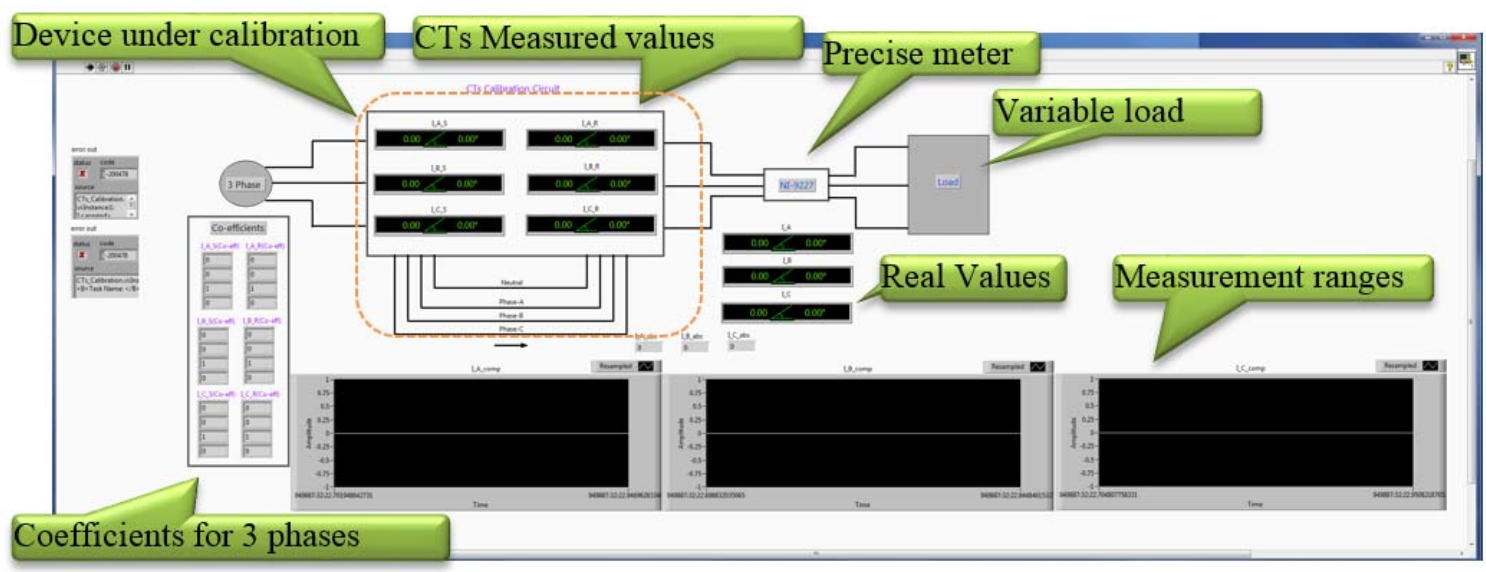

Figure 3.35 Developed CT calibration module

The complete design of measuring these quantities (using DAQs) is made in LabVIEW software.

The better solution would be to calibrate all the CTs according to the required output. The output measured from the CTs is sent through a MATLAB script node in the LabVIEW, where the code calculates all the polynomial co-efficient from the data obtained from the CTs (through interpolation). This polynomial is the inverse of the actual polynomial obtained from the data (Figure 3.10).

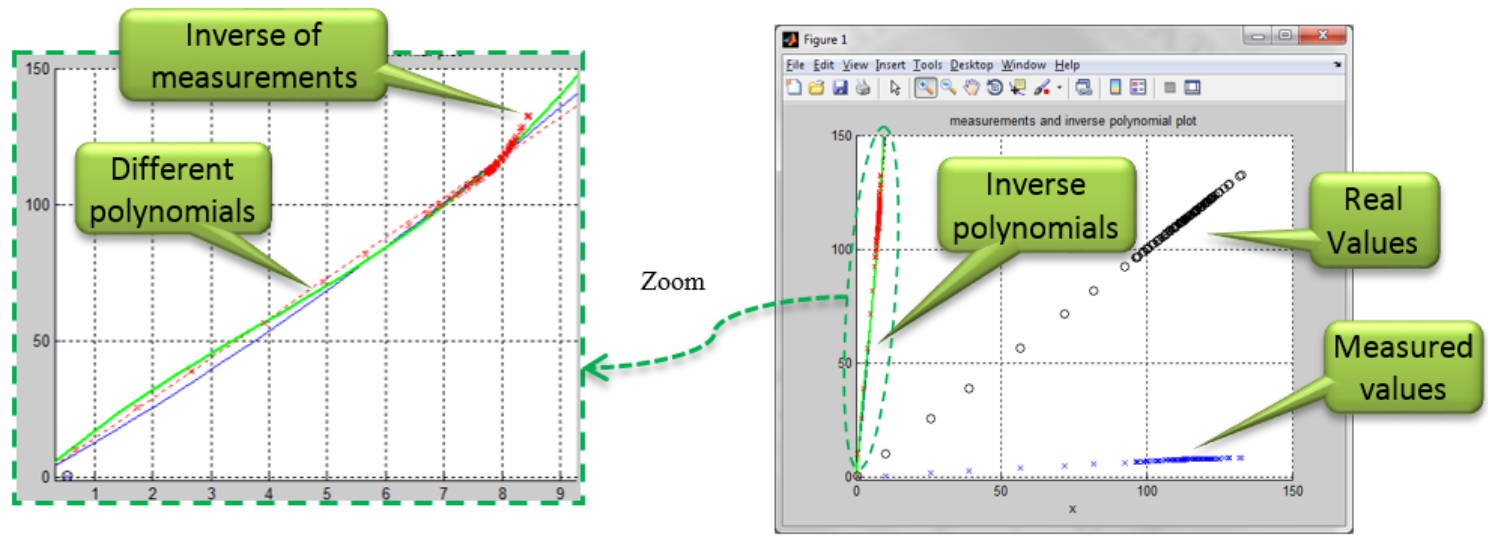

Figure 3.36 Interpolation of linearization inverse function 
If the CTs are working ideal, the curve would be a straight line. But here due to the non-linear operation of CTs, the output is a polynomial constructed from the data points. So by taking these co-efficient the deviations in the measurements of the CTs are taken and calibrated.

The actual correct measurements are taken from two National Instruments DAQs, the precise voltmeter is NI- 9225 which measures the voltage directly like a voltmeter and the precise ammeter is NI-9227 which measures the current directly like an ammeter. These measurements are observed in the LabVIEW. The measurements observed from the CTs and PTs are taken from the DAQ NI-9206(Ethernet) into the LabVIEW. These are being compared with those measured from the NI-9225 and 9227.

Depending on the order of the polynomial, the interpolation technique results will be different. Hence, it is important to know which polynomial to be used and for what range it would work. This was depicted in an example in Figure 3.37.

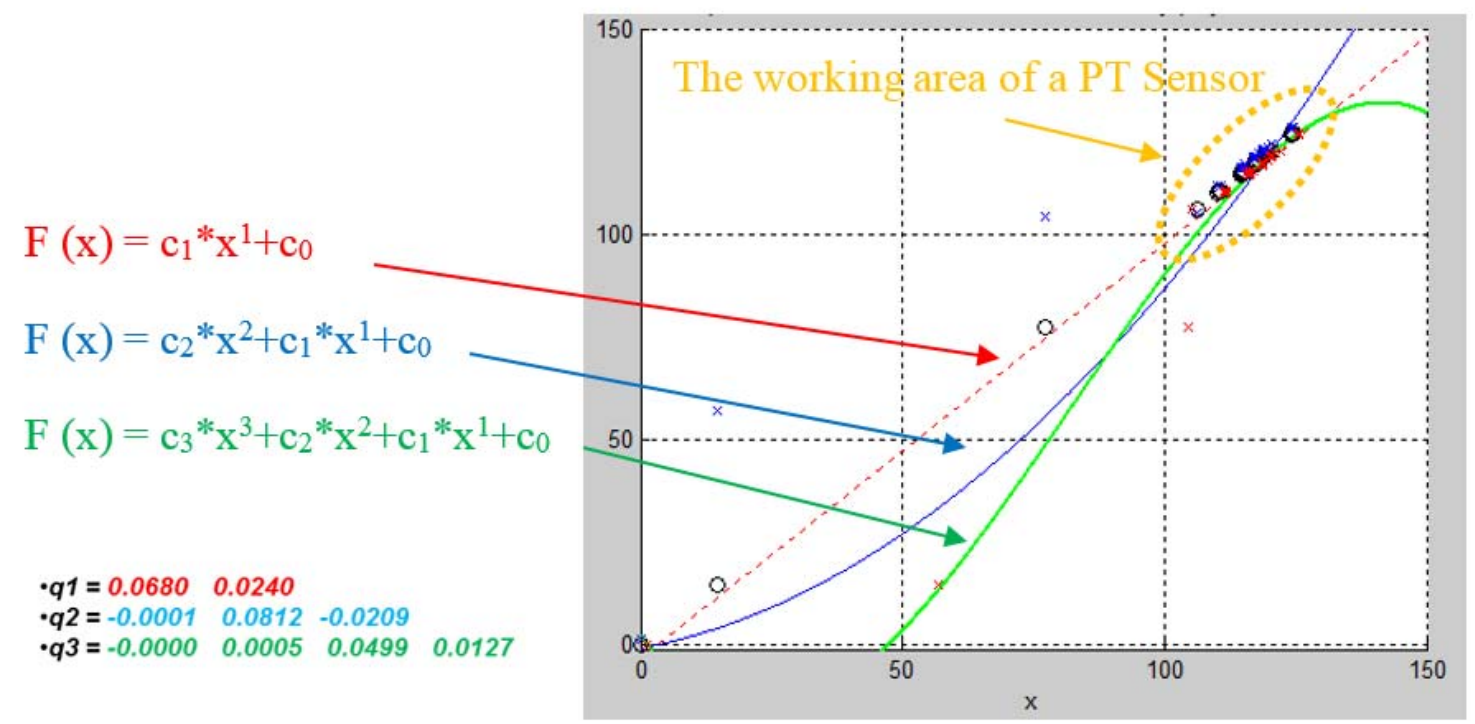

Figure 3.37 Applying different orders of polynomial functions for linearization 
It is important to know that in what range this sensor is going to work. For example, the PTs are usually working around nominal voltage (1 per-unit) e.g. they needs to be accurate in the range of 100-130V for a distribution level voltage sensor. 


\section{IMPROVEMENT OF MEASUREMENT SYSTEM THROUGH STATE}

\section{ESTIMATION}

State estimation plays a vital role to prepare the current state of the system based on the measurements and prepares input data for power system applications. This requires robustness, availability, maintenance, and of course accuracy. The state estimation's goal can be categorized as follows:

- To find state of the system for the positions when measurements are not available

- To keep the precision and the accuracy of the system

- To find the bad data and remove or replace them with pseudo estimations

With the recent developments in faster communication and using synchronized measurements and Phasor Measurement Units, performance of state estimation algorithms can be enhanced in terms of precision and integrity. There are also several methods of integrating phasor measurements in power systems.

In distribution systems 3-phase S.E. is being discussed in several articles [76][79]. Although they can solve the S.E. in 3-phase, but due to the long timing of calculations and rarely installation of PMUs in the distribution network, they are not so applicable and the positive sequence of the system is still the main source of measurements in S.E. applications. 


\subsubsection{Precise Measurements and the Benefits for SCADA}

Phasor measurement units are the most beneficial to the state estimation (S.E.), compared to the traditional measurements, when they are not reliable or available altogether. They can be used to directly extract the state of the system, even though poor calibration of heavily weighted PMUs can degrade the overall S.E.

Time synchronized measurements in power systems have the history started in 1970s [34], [36]. For synchronization, they include the time sources, such as pulse, radio, and Global positioning system (GPS) recently. Their time domain signal information, which is obtained by the sampling, aligned with the time information, which is obtained from GPS, and this provides a measurement system in digital relays, which delivers a very powerful tool, and capable of creating accurate measurements for the analysis of power systems. The first PMU was built in 1980s [36]. During that time PMUs were mostly being used for analyzing power system problems, wide area measurement systems in post processing analysis, and assessment through archived/recorded data.

Nowadays, improvements of communication system revolutionary changed the analysis domain. PMUs have been installed widely and their capability have the potential for real-time wide area digital data collection. With PMUs accurate time-stamped measurements, a synchronized state of the system (voltage and current magnitude and phase data) is available.

State estimation can be one of the real-time applications of PMUs. They find the positive sequence of the voltages and the currents synchronously and directly, and they can effectively change the state estimation into state measurement[34]. 
In addition to direct application of PMUs in S.E., PMUs are used to correct the network errors in S.E. algorithm too[80]. In [40], it is mentioned that mixing Phasor measurements with traditional measurements and then solving the extended problem by iteration techniques are not the best solution and it takes longer times to converge; Instead it's better to conduct the state vector estimated by the traditional measurements and afterward, use them in the linear post processing step along with PMU measurements.

Another reference discussed about practical approaches to state estimation on radial distribution systems and suggested that the feeder topology to be more important than the classical S.E. [79].

By using PMUs in [81], synchronized measurements, with the same time stamp as SCADA, are being used in traditional S.E. measurement set. For this case a change in Weighted Least Square (WLS) method is required to support the Phasor data. It requires adding some rows to the Jacobian matrix for the additional phasors. This method also has some issues, such as selection of proper reference, but again this method is not good enough due to larger matrix calculation and timing of calculation process [40].

The benefits of PMUs for state estimation application in power grids and also their impact on reliability of grid security was thoroughly investigated in [82]-[85]

In regard with the researches involving PMUs and S.E. following articles has discussed about the technique and comparison of optimal PMU placement algorithms for application in power system state estimation[86]-[88].

Implementation of PMUs for applications in S.E. can be divided into three major categories: 
1- Direct employment into classical S.E. formulation to improve overall performance. It is done by choosing the PMU as an accurate measurement with smaller error, and consequently, higher value in the weight matrix[89].

2- Decoupling PMUs from traditional measurements to improve accuracy and better bad data detections[90].

3- Identification of the line parameters by using the PMUs at the both ends of transmission lines, to get the parameters dynamically[91].

\subsubsection{Classical State Estimation Procedure Overview}

In order to understand the contribution of this study, let us first introduce the classical state estimation procedure. The S.E. basis was first introduced in 1970s [92]. They used SCADA measurements such as breaker status, tap positions, bus voltages, MW, MVAR, voltages of units and also the power and the currents of transmission lines and transformers.

The algorithm for a complete state estimation has 4 steps including network topology analysis, observability analysis, state estimation computation, and bad data detection.

A necessary requirement for an observable system is that the number of available measurements is equal or larger than the number of estimated variables. This verification can easily be made at the beginning of every state estimation.

But it can also happen that only parts of the network are observable and some other parts of the system are not observable even if the total number of measurements is sufficient. Hence, it is not only important that there are enough measurements, but also that they are well distributed in the network. 
The Complete process is presented in Figure 4.1, but in this work the concentration is not on the observability and pseudo measurements, and they are not considered.

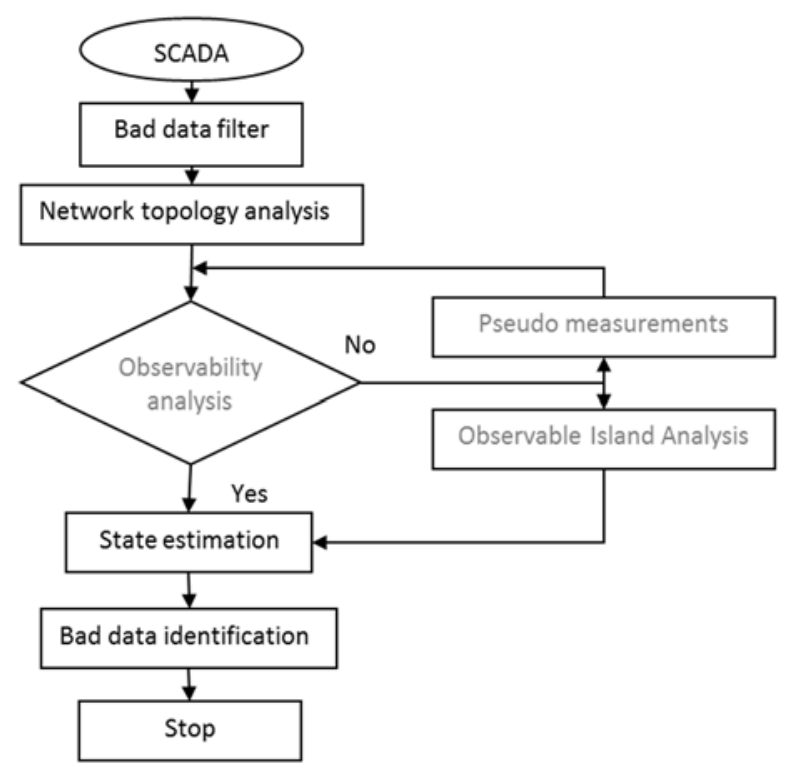

Figure 4.1 A Complete state estimation process

In order to do so, the existing topology of the system is a requirement. Thus, a topology processor will prepare the present status of the bus-branch data for the S.E. software.

It is assumed that states are $x$, errors are $e$ and measurements are $z$. so

$$
z_{i}-h_{i}(x)=e
$$

In which $x=\left(\theta_{1} \theta_{2} \ldots \theta_{n} U_{1} U_{2} \ldots U_{n}\right)^{T}$ and $h(x)$ is the measurement function, which relates the system state vector $x$ (voltage angles and magnitudes) to measured quantities $\left(V_{i}, P_{i}, Q_{i}, P_{i j}, Q_{i j}\right)$.

An important assumption for this method is that the measurement errors are stochastic with Gaussian probability distribution, which has zero expected values and 
independency. Only in this case we can find the most likelihood state of the system based on our measurements.

The assumption (zero expected value of errors) invents a minimization problem and by solving it, we can find the states by just having the measurements. This minimization usually solved by weighted least square method (WLS). The theory of WLS S.E. is very well known [93][92] [81]. The minimization function is

$$
J=\sum_{i=1}^{m} W_{i i}\left[z_{i}-h_{i}(x)\right]^{2}
$$

In which $W_{i i}$ is the weight and it is inversely proportional to error variance:

$$
W_{i i}=\frac{1}{\sigma_{i}^{2}}
$$

In order to find the minimum of that function, the first derivative should be equal to zero. Also for more simplification, the Taylor series of that function around $x^{(k)}$ is assumed:

$$
g(x)=g\left(x^{k}\right)+G\left(x^{k}\right)\left(x-x^{k}\right)+\cdots=0
$$

Furthermore, the higher order terms are neglected. Finally the function is changed to:

$$
g\left(x^{(k)}\right)+\frac{\partial g\left(x^{(k)}\right)}{\partial x}\left(x-x^{(k)}\right)=0
$$

By using Gauss-Newton iterative method the equation above can be solved and $x$ can be found. 


$$
x^{k+1}=x^{k}-\left[G\left(x^{k}\right)\right]^{-1} \cdot g\left(x^{k}\right)
$$

Simplification leads to Normal Equation:

$$
g\left(x^{(k)}\right) \Delta x^{k+1}=H^{T}\left(x^{(k)}\right) \cdot W \cdot\left[z-h\left(x^{(k)}\right)\right]
$$

In which:

- $\mathrm{k}$ is the iteration index and $x^{k}$ is the estimated value at the $\mathrm{k}^{\text {th }}$ iteration.

- $G(x)=\frac{\partial^{2} J(x)}{\partial x^{2}}=H^{T}(x) . W \cdot H(x)$ is the gain matrix, which is always positive, sparse, definite and symmetrical for observable systems.

- $\Delta x^{k+1}=x^{k+1}-x^{k}$ is the update of solution at the iteration $k$

- $H(x)=\frac{\partial h(x)}{\partial x}$ is the Jacobian matrix of measurements

Solving the last equation iteratively until reaching the $\Delta x^{k+1}<\epsilon$ will be resulting in the final S.E. solution.

The Gain matrix is not usually inverted because of large sparsity. Instead, the equation is solved by sparsity techniques, such as decomposition of $G$ into triangular factors and using back/forward substitution at each iteration (or Cholesky factorization).

There are also newer factorization techniques such as Givens Rotations, which helps to have covariance matrix $R$ elements for a better solution of S.E.

Over the years, many developments have been performed to the S.E. method, but traditional WLS S.E. still remains the best and accurate method of estimating the state of the power system in EMS.

In section 4.1.3 available Hybrid S.E. was explained in details in addition to the S.E. technique. 


\subsubsection{Hybrid State Estimation}

In hybrid methods, the S.E. is improved by using both the traditional measurements and the PMU results. In this case, outcomes of the traditional S.E. in the first step will be used, along with PMU measurements, in the second step, which is a linear S.E.; by putting the values together, we can formulate the S.E. as:

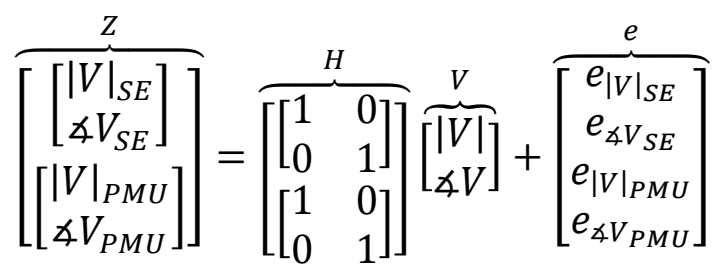

In which $Z$ is the measurement matrix, $H$ is the Jacobian matrix and all values are represented in the polar basis.

This method will be improved on our proposed method to be able to solve our problem, which will be explained in the next section.

\subsection{PMU for State Estimation}

The goal of such work is to merge synchrophasor data, along with conventional measurements, to improve the state estimation results for different real-time applications. This case can be extended to a situation when some localized PMU encounter timing reference problems, like missing a GPS clock, deviation in the timing, or missing the connection to the IRIG signal. In this case the power system can be assumed as the following and represented in the Figure 4.2.

Another example, with the same behavior, is in the industrial systems, when the system consists of several data acquisition devices, with multiple inputs (ADCs), which are internally synchronized, but DAQs have no common triggering signal. To 
synchronize those systems and get to a global state of the system, a merging technique for synchronizing the DAQs are necessarily required (Figure 4.3).

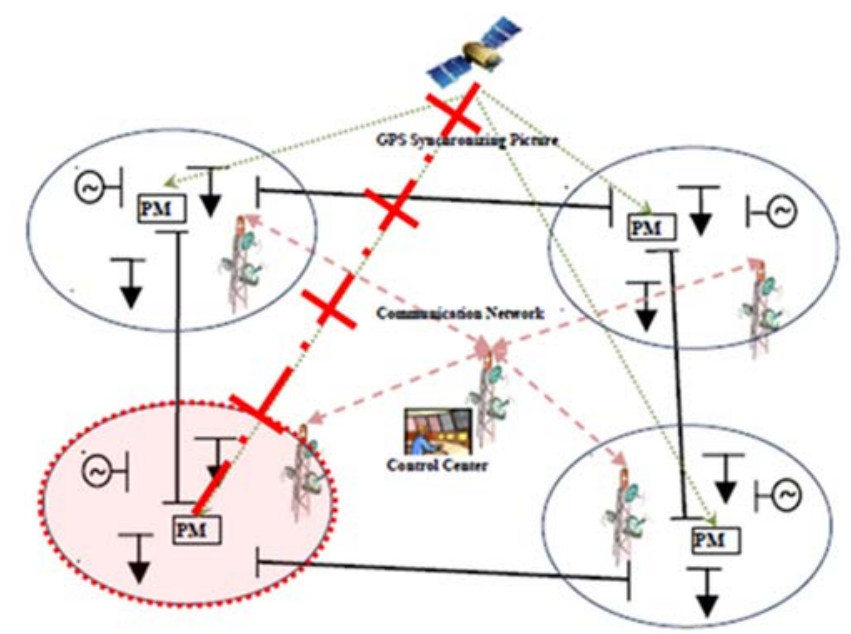

Figure 4.2 Local isolated/deviated synchronized measurements in global power system

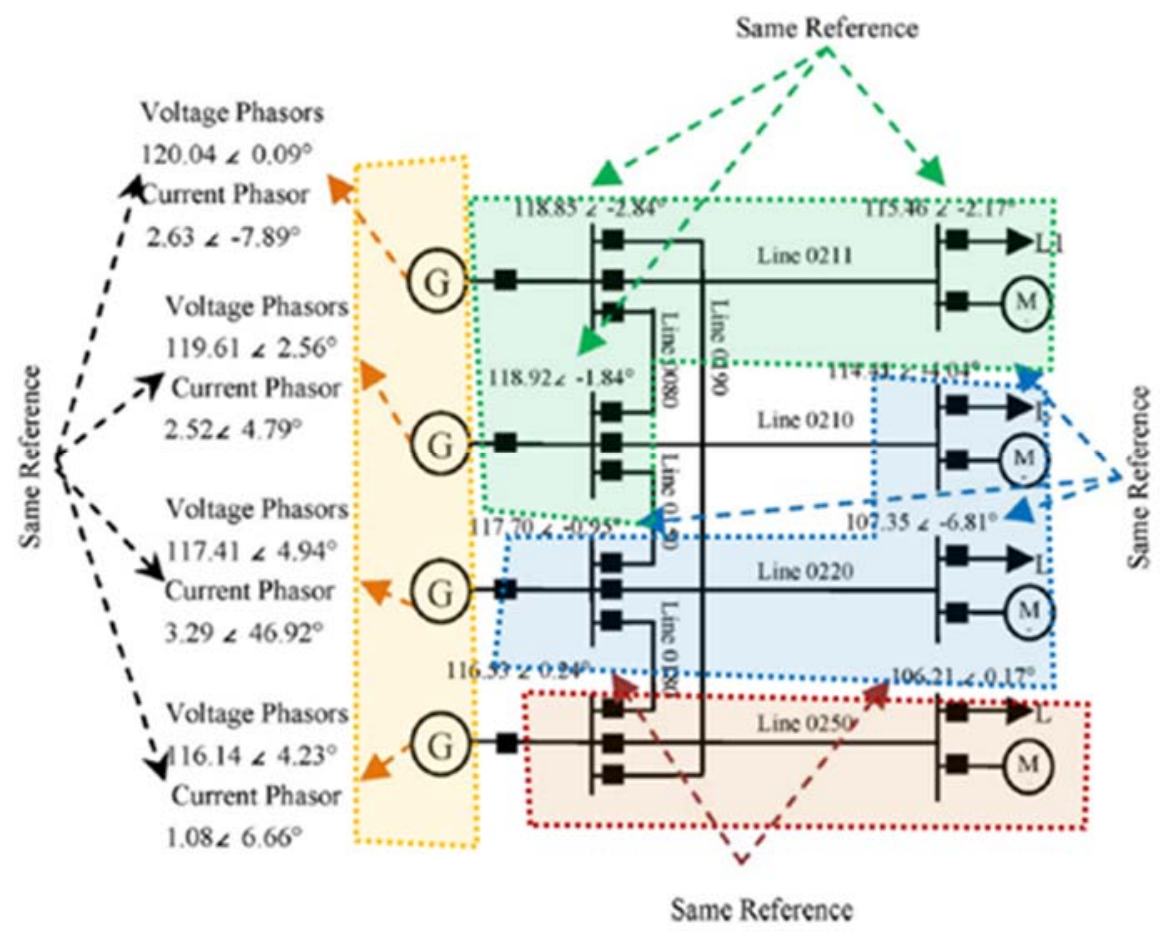

Figure 4.3 Separation of synchronized measurement (grouped by color) 
For the case of disconnected GPS Clock in a PMU, although PMUs are usually equipped with internal clock, but long time operation of standalone time reference, without synchronization with GPS time reference, may deviates the time reference and creates errors in the phase calculation of voltage and current waveforms.

In the next sections, a new method will be introduced to exclusively prepare a phase difference compensation to bring the two PMUs into the same reference.

\subsection{Suggested Methodology to Reduce the Estimation Error}

The proposed method is improving the hybrid state estimation method by implementing multiple PMU groups in the measurement and Jacobian Matrix.

It will change the equation to:

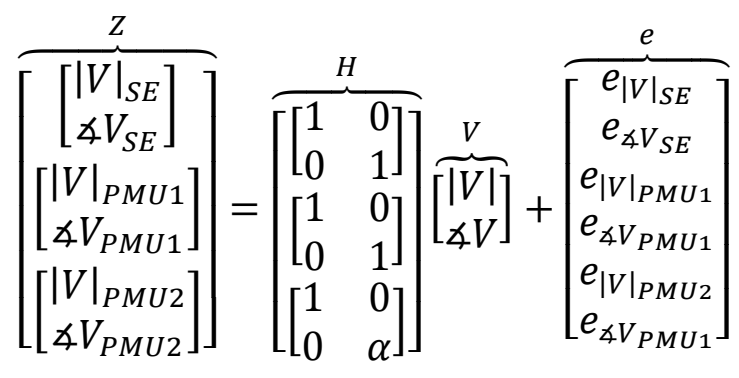

In the above equation, it is assumed that the system has two time references grouped by PMU1 and PMU2.

Since the system has two time references, states should be in a unique reference.

This is possible when the phase shift of $\alpha$ maps the PMURef.PMU2 to PMURef.PMU1. For this purpose, another Minimization methods needs to be performed. For this case the timing references should change to satisfy the following equation:

$$
\Varangle V_{\text {Ref.PMU1 }}-\Varangle V_{\text {Ref.PMU2 }}=e
$$




\subsection{Real Time Implementation on Experimental Setup}

The small scale power system, which was used to verify the method, will be explained in details later in chapter 7.

In order to solve the S.E. problem, current topology and physical parameters of the grid are needed. Transmission lines' physical parameters are indicated in Table 4.1. The parameters of the Pi-model of transmission lines can be found through synchronized measurements located on the both ends of each line, based on the technique represented in Section 9.5.1.

Table 4.1 Transmission Lines Parameters and Their Connectivity

a.

\begin{tabular}{|c|c|c|}
\hline \multicolumn{1}{|c}{$\mathrm{R}$} & \multicolumn{1}{c}{$\mathrm{X}$} & \multicolumn{1}{c|}{$\mathrm{B}$} \\
\hline 0.209799 & 0.426412 & 0.002462 \\
\hline 0.088835 & 1.109104 & 0.003633 \\
\hline 0.110941 & 1.121358 & 0.003646 \\
\hline 0.111512 & 0.956026 & 0.003631 \\
\hline 0.111674 & 1.020069 & 0.003615 \\
\hline 0.133233 & 1.220625 & 0.003612 \\
\hline 0.110239 & 1.105841 & 0.003615 \\
\hline 2.286574 & 3.225841 & 0.002459 \\
\hline
\end{tabular}

b.

\begin{tabular}{|c|c|}
\hline & End \\
\hline 320 & 40 \\
\hline 320 & 380 \\
\hline 380 & 260 \\
\hline 260 & 110 \\
\hline 380 & 50 \\
\hline 260 & 140 \\
\hline 320 & 110 \\
\hline 110 & 130 \\
\hline
\end{tabular}

\subsection{Integration of Real-Time Data Acquisition for State Estimation}

The real-time information which is used in the state estimation process is coming from the DAQ measurements, through the calculation sub-VI which is explained in Chapter 3. In addition to data acquisition measurement, an interface was developed by other researchers in ESRL to provide the measurements from the actual phasor measurement units (commercial PMUs) (Figure 4.4). 


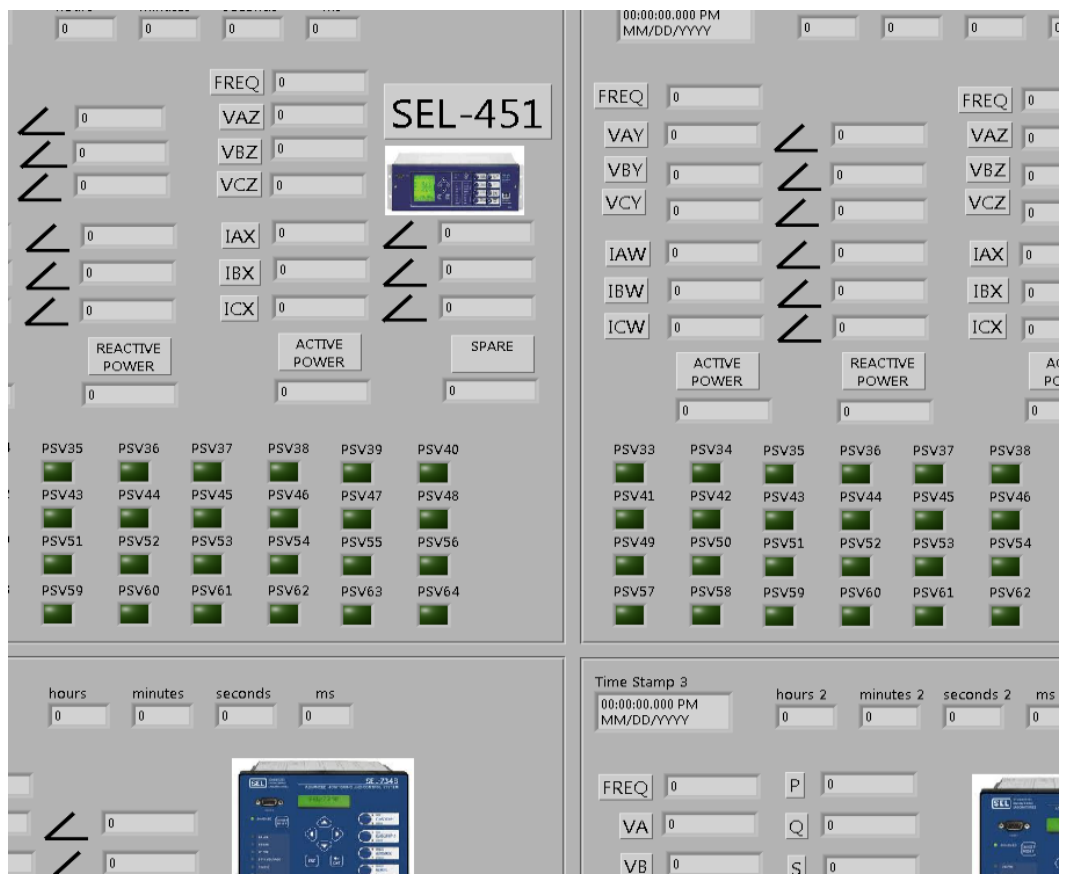

Figure 4.4 PDC interface in LabVIEW for SEL PMUs and protection relays (courtesy of ESRL)

\subsubsection{State Estimation algorithm developed in Matlab}

The state estimation program is developed in Matlab software and that is due to the processing and computational advantages especially in the matrix mathematics.

The program is using the following information is the input in the calculation process:

1- Bus status:

- Which has the information of buses which includes bus number, bus voltage magnitude, bus voltage angle, bus measured voltage magnitude, bus measured active power, and bus measured reactive power.

2- Line status: 
- Which has the information for the transmission lines including start bus, end bus, resistance, reactance, shunt admittance, tap changer ratio, transferred active power, and transferred reactive power.

3- Shunt status:

- Which has the information of parallel (shunt) components in each bus and includes bus number and shunt value.

4- Bus weight data:

- Which has the weight values for each measured parameter of buses, such as voltage, active, and reactive power measurements.

5- Line weight data:

- Which has the weight values for active and reactive power measurements transferred in each transmission line.

Based on the algorithm of classical state estimation Section 3.3, the state estimation has the following algorithm (Figure 4.5), but in this work the network observability check/correction is ignored.

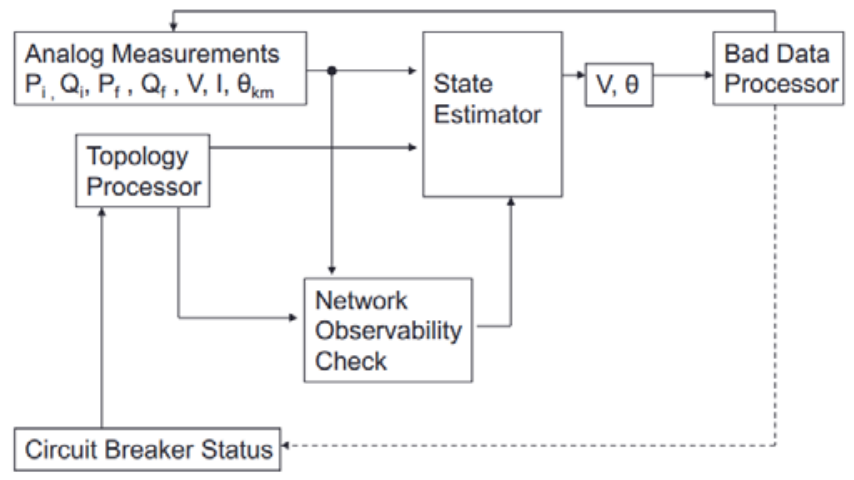

Figure 4.5 State estimation program 
The developed state estimator program in Matlab environment has an engine which performs the following plan:

1. Build Y-bus

A. insert shunt load into y-bus

B. take care of tap positions of transformers

C. take care of constant impedance loads

D. separation of $G$ and $B$

2. Find the measurement mismatch

A. find the mismatch error vector

a- calculate power injection

b- calculate reactive power injection

c- find flow of active and reactive in lines

$d$ - find the Jacobian matrix

i. find sub matrixes of real power injection and reactive power injections

ii. find the real current flow and reactive current flow in lines

e- Birth of Jacobian matrix

3. Define the gain matrix

A. apply weights to measurements

B. $\operatorname{Gain}=H^{\prime} * W^{*} H$

C. Birth of measurement mismatch error matrix

a- SMM=Gain- ${ }^{*} H^{*} W^{*} m m$ 


\section{Find the new states}

\section{A. Find the new voltage states \\ B. Find the real/reactive power calculation \\ C. Find the real/reactive flow in lines}

The parameters of the above program engine was defined earlier in section 3.3.

\subsubsection{Integration of Real-Time State Estimation (in LabVIEW Environment)}

The LabVIEW is the heart of state estimation. It provides a shared memory for data acquisitions, calculates the electrical parameters of each node of the system, present it to the operator, and by interconnection to Matlab engine, provides the values for state estimation program.

The front panel and the block diagram on the Virtual Instruments (VIs) developed in LabVIEW are presented in Figure 4.6 and Figure 4.7.

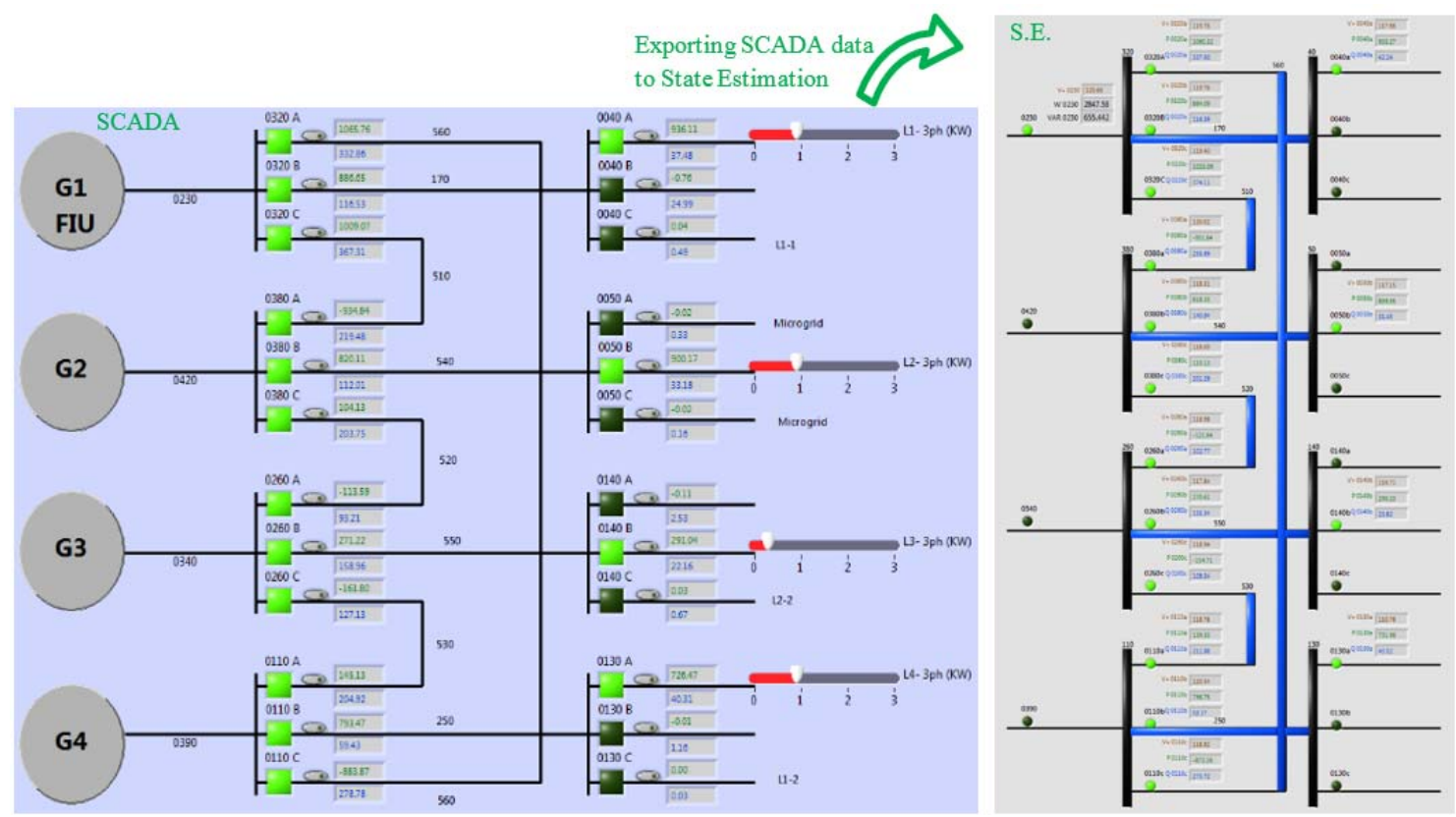

Figure 4.6 Exporting the SCADA information to state estimation interface 


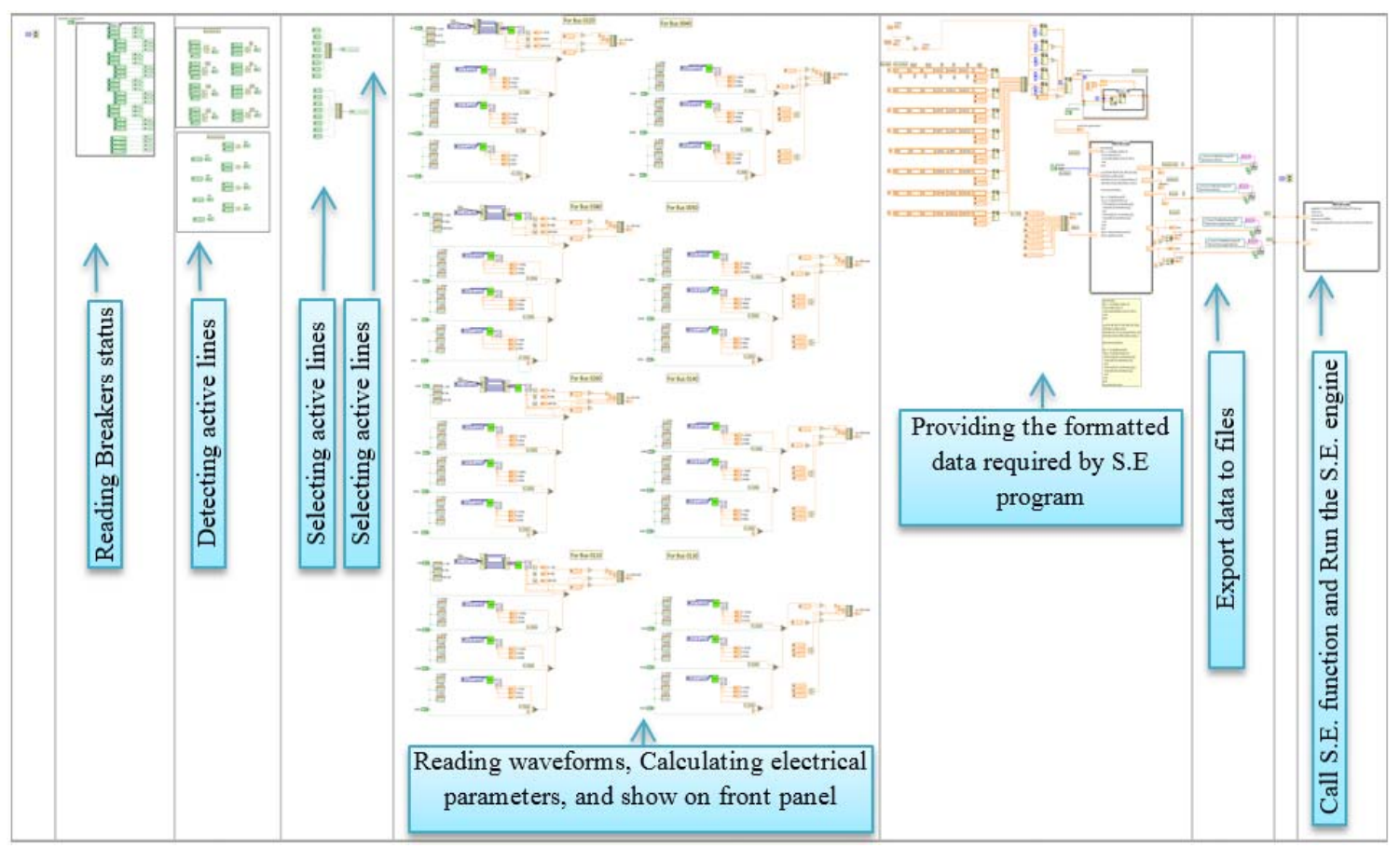

Figure 4.7 Sequential block diagram of state estimation interface

\subsubsection{Performing State Estimation in the Commercial Software (DIgSILENT PowerFactory)}

DIgSILENT PowerFactory is a power system analysis software package for use in analyzing generation, transmission, distribution and industrial systems. It includes the full range of functionalities as well as optimal load flow and state estimation.

The interconnection software platform can be provided to have a close to realtime analysis, by using LabVIEW as the data collector and DIgSILENT as the analysis engine.

The ONLINE ANALYSIS VI is designed to prepare the interconnectivity between the two software, and the front panel is represented in Figure 4.8. 


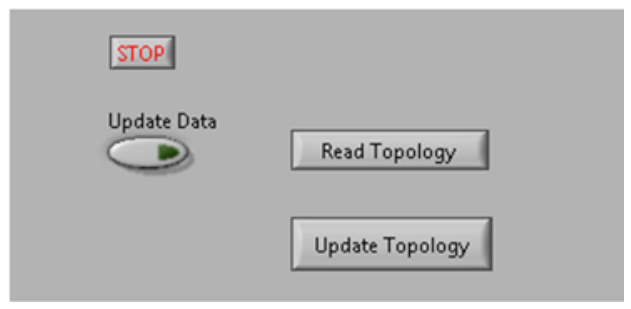

Figure 4.8 Front panel of online analysis interface

In order to use such technique, it is required to make a general model of the power system in DIgSILENT and update the values through LabVIEW continuously.

As an example, the information of the power grid in Figure 4.6 was applied to the load flow and S.E. engine in DIgSILENT and the results are presented in Figure 4.9.
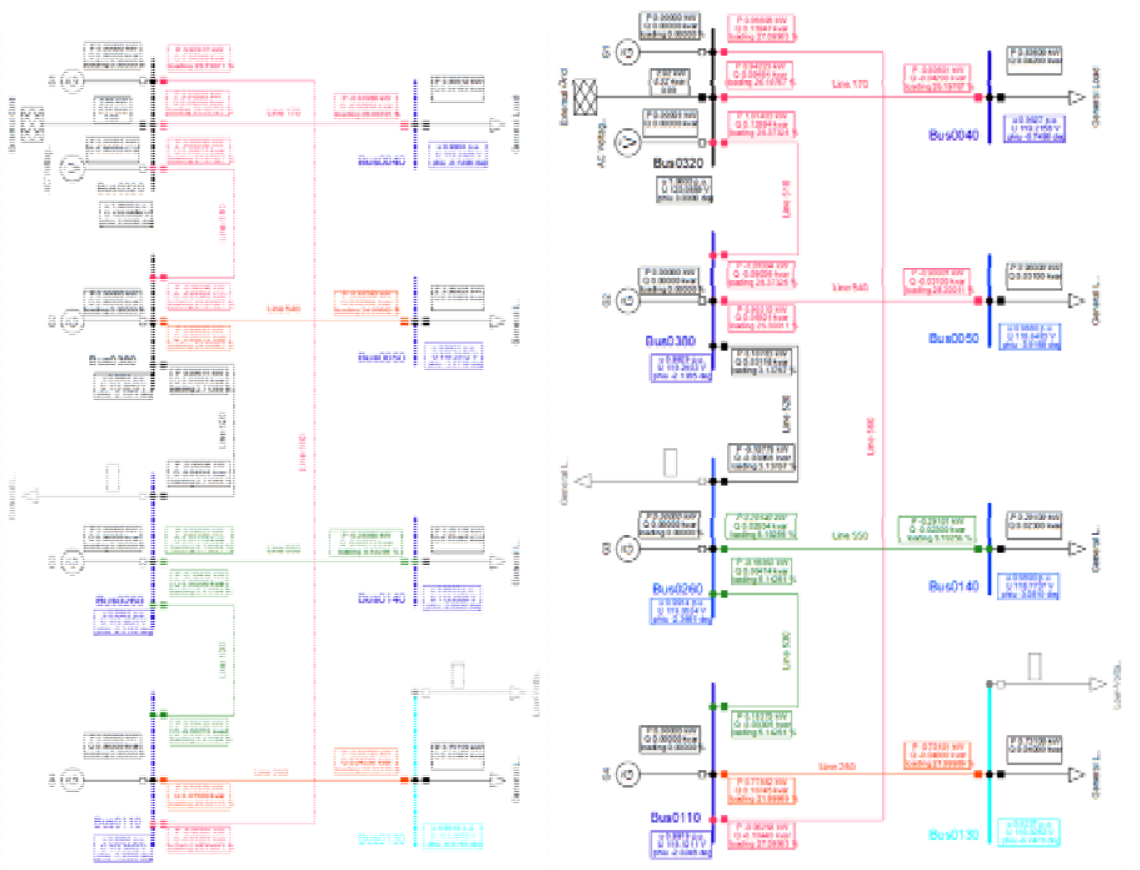

Figure 4.9 Load flow results (Left) compared to state estimation results (Right) 
The results of load flow and state estimation are very similar in this case. The main difference is because of voltage drop of solid state relays which are used as breakers in the real time emulated power system (smart grid testbed).

\subsection{Post Processing: Detection and Elimination of Bad Data}

The SE may also provide the function of detecting, identifying, and eliminating gross measurement errors throughout the system, in addition to filtering small random measurement errors. These large errors usually occur when meters have biases, drifts/shifts, or are simply incorrectly configured/set.

For bad data detection it is recommended to categorize the data as they appear in cases where there is a single bad data with large error, or the case where there are several bad data. The case of multiple bad data can further be classified into three sub-categories: non-interacting, interacting but non-conforming, and interacting as well as conforming [94]. Non-interacting bad data have weakly correlated residuals, and are therefore are not significantly affected by each other's residuals. In the case of interacting multiple bad data, good measurements can appear to be in error if they are strongly correlated with bad data. In addition, conforming errors appear consistent with each other, and therefore it is difficult to identify the measurement in error. After running the state estimation process the results for the abovementioned example can be reported as the following: In this example, the S.E. results show that 4 active power measurements $(\mathrm{P})$ of branches are failing. 


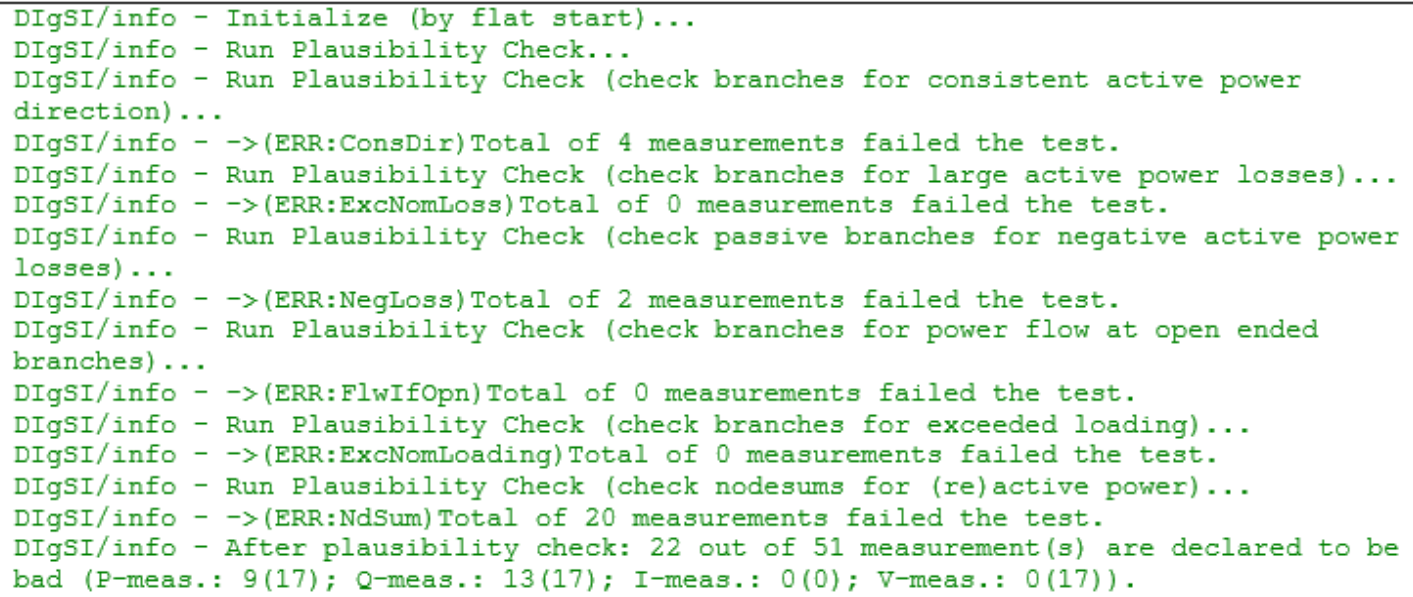

Figure 4.10 An example of pre-processing in state estimation

The aforementioned pre-process concludes that 22 out of 51 measurements are not considered good for S.E. After S.E. process converges, which is in 5 iterations in this case, S.E. results will be presented as in Figure 4.9.right and the report of process concludes:

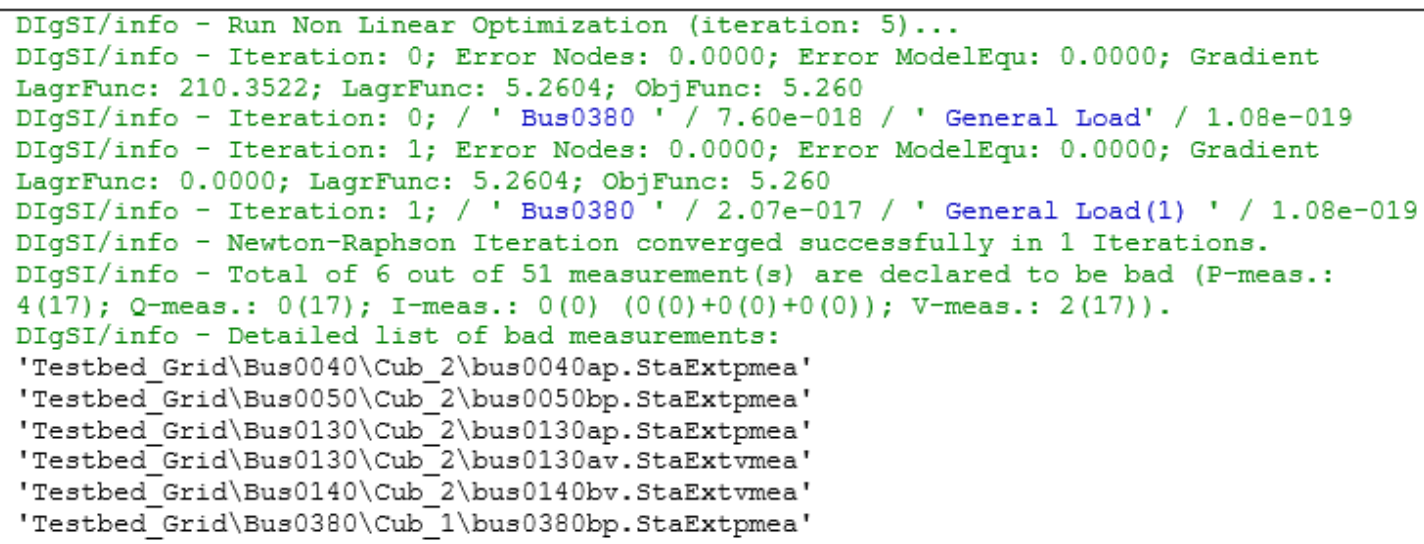

Figure 4.11 An example of post-processing and bad data detection.

The report state estimation results can conclude bad data detection according to accuracy exceeding factors for active power, reactive power, voltage magnitude, and current magnitudes. 


\subsection{Conclusion}

In this chapter a practical and implementation of a wide area state estimation was explained. A Matlab program is developed to perform an automated state estimation. This Automated system can perform the S.E. process go well together with the LabVIEW integrations and the real-time SCADA. By using this platform, it is possible to integrate the PMU reading for state estimation process and for special cases when a group of PMU readings are deviated from time synchronism. It is also suggested to apply an optimization technique to eliminate such errors within the hybrid state estimation method.

In addition, a new interface was developed to perform the S.E. and load flow in a third party software package (DIgSILENT). The results were presented to show the capability and interoperability of the developed system.

This work is accomplished in an experimental laboratory platform to show the feasibility of the developed S.E. technique, using measurements through DAQs and conducted to form a like-wise real-time measurements of the power system. 


\section{TRUSTED SENSING BASE FOR SECURITY OF POWER GRID}

\subsection{Introduction}

Trusted operation of smart grid critical infrastructures requires effective and secure solutions for monitoring and control of such cyber-physical platforms. However, due to the insufficiency of the deployed security solutions, there have been several largescale and targeted intrusions and outages. A recent and well-known example is the Stuxnet computer worm [95], which targeted Siemens industrial software used to control nuclear fuel processing plants. The worm exploited several extremely complicated cyberattack vectors, including four Windows zero-day vulnerabilities [96], to sabotage a suspected uranium processing facility. The scale and complexity of the attack clearly demonstrated the need to fully monitor cyber-physical critical infrastructures in real-time

for both accidental and malicious failures. Such monitoring would allow the power grid operators to take quick responsive and corrective actions if the power grid is under attack or has experienced failures.

Comprehensive monitoring of the power grids require trusted sensors; otherwise, adversaries may exploit the introduced attack surfaces to cause false data injection attacks [62]. In particular, attackers compromise and take control of a specific subset of power system sensors, e.g., phasor measurement units (PMUs), and corrupt sensor measurements such that they could evade the state estimation procedures within the control centers. 
There has been several previous works proposed to protect against data injection attacks that could be categorized into two main groups. First, intrusion preventive control theoretic analysis solutions [97] have mostly considered the power system as a linear control system and investigated how its observability could be affected by any compromised subset of PMUs. If the attacker manages to compromise the system observability from the viewpoint of the state estimation server at the control centers, the attackers could potentially mislead the power operators at control centers regarding the current actual state of the underlying power system through sending correlated corrupted PMU measurements. Second, intrusion detection solutions have been introduced to identify such measurement corruption attacks. Combinatorial residual analyses [98] aim at detection of the subset of compromised PMUs by considering and analysis of all possible subsets during the wide area state estimation. The measurement subset that deviates the estimated state the most is marked as the compromised PMU subset. Other security-aware intrusion detection solutions [99] have made use of cyber-side security sensors, i.e., Intrusion Detection Systems (IDSes), alerts regarding the compromised host systems in the power grid. As the measurement corruptions are mostly caused by the compromised host systems, IDS information could be used to narrow down the search process during the combinatorial analysis of false data injection attacks. Both of the abovementioned techniques assume that the attackers could corrupt measurements once they compromise PMU devices that is currently the case in practice as the measurements are accessible in plaintext form within the compromised PMUs. 
In this chapter, we extend our work to propose TSB, a trusted sensing base for smart grid infrastructures. TSB that is a fundamental practical solution to protect critical infrastructures against the malicious measurement corruption attacks. We design, implement and deploy a hardware power system sensor, e.g., current transformer, that encrypts the $\mathrm{AC}$ power signal within the sensor at the data acquisition point before sending it over to the A-to-D and PMU devices; therefore, the malicious parties with control over the PMUs will access only the encrypted measurements and hence the power operators would be able to detect any unauthorized manipulation of the measurements coming from the sensors. To that end, we make use of specifically the way current transformers are built in practice, i.e., current scale-down and voltage measurement of a fixed resistor within the sensor. TSB has two agents within the current transformer sensor and the control center. The sensor agent implements an AC signal encryption scheme using a synchronized micro-controller and a secure one-way hash function. TSB's control center agent on the other end is synchronized with the sensor and is responsible to reconstruct the decryption procedure that is used to retrieve the original measurements. Consequently, the measurements are kept secure (encrypted) all the way from the sensor to the control center and attackers are left unable to corrupt them within the PMUs.

TSB's contributions are as follows. It proposes a new system-level architecture for smart grid data acquisition to protect against false data injection and unauthorized man-in-the-middle measurement sniffing intrusions. It introduces a novel AC analog signal encryption scheme using keyed message authentication code functions that could be used in generic cyber-physical critical infrastructures. We develop a working hardware prototype of TSB and evaluate it in a real-world power grid test-bed including 
the complete data acquisition chain from sensors, A-to-D, PMUs, and the control center. More specifically, this method is built upon our past conference publication in terms of the following three extensions. First, we actually implemented the GPS synchronization framework in TSB with real-world GPS clock (SEL-2407 devices). Second, we made TSB completely autonomous through using more capable components such that the sensor could be deployed independently without the need to a real-time LabVIEW connection for hash function implementation among other computational tasks. Finally, we completed the data acquisition data flow chain all the way from the sensors to the control center via real-world phasor measurement units (SEL-421) and phasor data concentrators (SEL-5073). Consequently, we deployed TSB on a production-level setting and hence our results are all real with no simulation proving TSB's deployability in practice.

\subsection{Related Work}

Recently, there is an increasing interest in security incident prevention and detection in power-critical infrastructures. Cyber-side diagnostics mechanisms try to estimate the security state of a computer network [63]. Ten et al. [64] propose a vulnerability assessment technique to evaluate the vulnerabilities of SCADA systems at three levels: system, scenarios, and access points. Mohajerani [65] introduce a method to detect and improve the vulnerability of power networks against the intrusion and malicious acts of cyber hackers by calculating the risk of each asset being compromised. Both the abovementioned frameworks perform in an offline manner, and hence cannot monitor the system for malicious activities while it is in its operational mode. 
Additionally, there have been several solutions introduced to provide security to the substation-control center communication links [66]. For instance, Kang et al. [67] suggest an end-to-end encryption and key management solution for power grid infrastructures. Almost all of the previous solutions to employ cryptography to guarantee the overall infrastructure security fall short in two major aspects. First, it is practically hard to perform data encryption and decryption on measurement samples in real-time especially in large-scale power grid infrastructures with large amount of network traffic. Second, as the encryption is performed in substations after the measurements are sensed and sent out to the analog-to-digital devices, the proposed solutions are not resilient against analog-todigital device compromises. TSB provides encryption right at the sensing point to minimize the size of the trusted domain for the substation-control center communication security.

\subsection{Problem and Solution Overview}

We explain the threat model against which the TSB framework protects the smart grid infrastructures, and review the high-level architecture of our proposed solution.

\subsubsection{Threat Model}

With the recently increasing level of communication capabilities among power system components, e.g., phasor measurement units, and potential component accessibilities by external third parties, remote component compromises through cyber vulnerability exploitations calls for effective defenses against cyber adversaries. It is important to clearly describe the threat model before getting into the solution details. Throughout this solution, we assume that the (remote) attackers are initially residing in 
Internet, and are able to exploit network/device vulnerabilities and compromise all the communication and substation-level devices, e.g., power measurement units, which are accessible through Internet. However, in this method, the power control room remains trusted, and our objective is to provide end-to-end security for power system measurements which flow originating from the sensors and are received and processed in the control room facilities. We believe that the trusted control room assumption is rational given their usual strict security configurations and global access control policy enforcement solutions that are often missing in the substation-level infrastructures.

\subsubsection{High Level Architecture}

Traditionally, trusted computing base (TCB) solutions [100] for the system execution security act as execution trust roots and are designed as small trusted execution/computational resources that the whole system execution security depends on. Analogous to the TCB for execution security, TSB's objective is to provide data trust roots in power grid infrastructures by implementing security right before sensors acquire the data/measurements. In particular, the TSB framework develops new hardware data acquisition devices, i.e., extended current and voltage transformers, and encrypts analog measurements by modifying the way they are obtained by the transformer hardware.

Figure 5.1 illustrates the high-level architecture of the TSB framework and how it enhances the conventional power grid data acquisition infrastructures.

In this Chapter, we focus on the power line current and voltage measurements. Other power system parameter measurements can be enhanced using the TSB framework similarly. Traditionally, current transformers are in charge of scaling down the line current values and sending the acquired analog signals to the main unit, e.g., the power 
measurement unit, which has several important responsibilities. First, each PMU uses its Analog-to-Digital conversion module to convert the received continuous analog signals into digital samples.

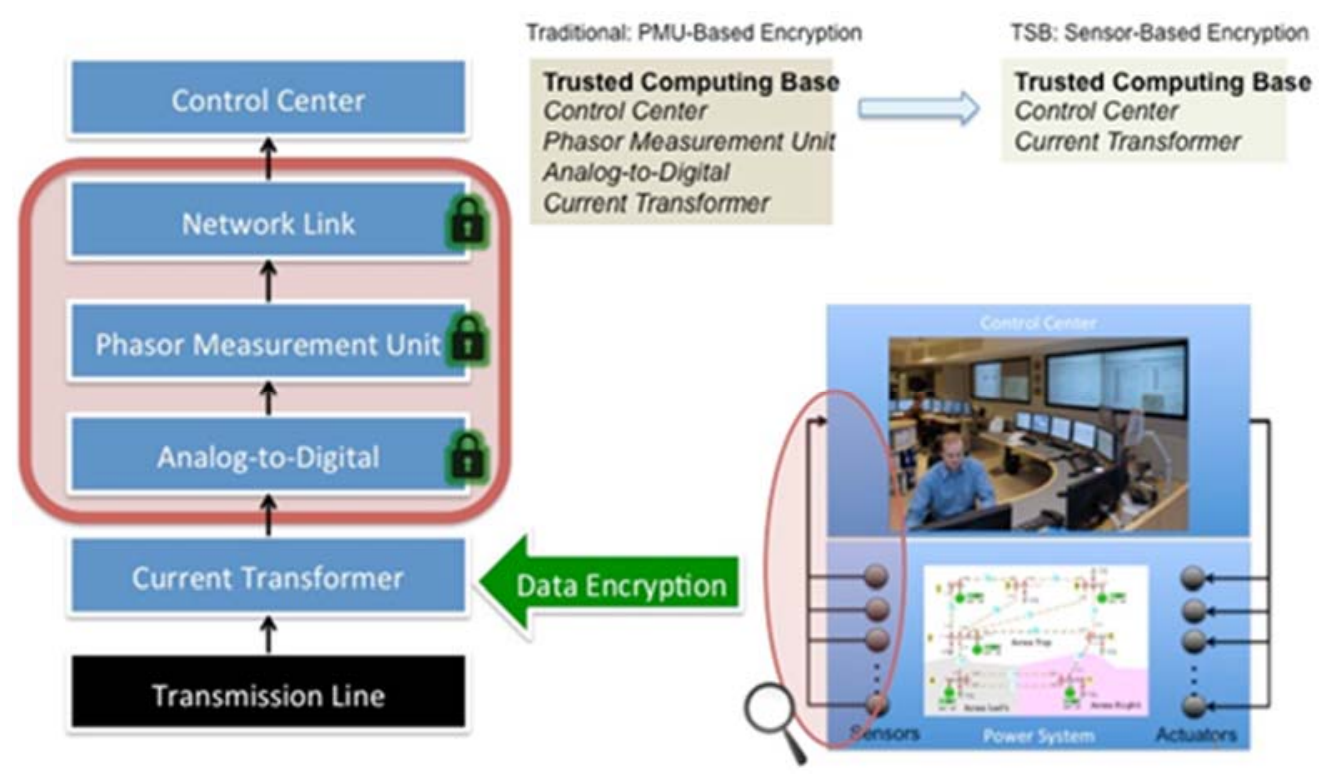

Figure 5.1 TSB high level architecture

For security purposes, the digital samples may then get encrypted within the PMU unit. Finally, through a wireless or wired network link, the encrypted data are sent out to the data aggregation centers, and eventually, the control rooms. Upon receiving the encrypted digital samples, the decryption module decodes the encrypted samples and turns them in to the state estimation server for wide area monitoring and power system stability control purposes. As discussed, the PMU units perform many complicated data processing, they can be connected remotely through the network links by the system operators for maintenance purposes. Such a remote connection capability, while being a very useful and convenient facility, opens a new wide attack surface for the malicious parties. In particular, a PMU firmware with software vulnerabilities can be accessed and 
compromised by the attackers, who can then gain control over the unit and conduct serious consequences. As a case in point, the attacker can intercept the power measurements and inject false power data before them getting encrypted by the unit. As result of the intrusion, the control room, and hence the state estimation server, receive corrupted power measurements from the compromised PMU units. Consequently, the power system state estimate, which is the estimation server result and is monitored by system operators, can be controlled by the adversaries indirectly. Liu et al. [3tsg] investigate how different combinations of the compromised sensing units enable attackers to make the system look different from its actual state to the operator.

TSB raises the security for the distributed power grid data acquisition infrastructures. The main objective of the TSB framework is to provide a trusted sensing base by pushing the data encryption modules to within the end sensing point, where the analog data acquisition procedure is performed. Through minimization of the trusted domain size, i.e., the current transformers, the TSB architecture significantly limits the substation attack surface to solely intrusions where the attackers own physical access to the sensing platforms within the substation. In other words, remote attackers who gain control over the PMU devices will no longer be able to get access to the actual measurements, because the analog signals will have already been encrypted within the current transformer before getting to the PMU device. As both ends know the encryption algorithm, the control center's communication server decrypts the received digital data accordingly so that the operators will see the system's actual status on the HMI screen. The design philosophy is analogous to the trusted computing base concept [101] where a minimal trusted domain is designed for execution to minimize the system's attack surface. 


\subsubsection{Sensor Side Signal Encryption}

TSB performs the data encryption at the sensing (before data acquisition) end point to make the false data injection attacks through cyber compromises infeasible. We explain the encryption algorithm that TSB implements in each sensor, e.g., current transformer, using an inexpensive circuit to maintain end-to-end security guarantees. TSB encrypts continuous analog power line signals before they are sent to the PMU unit, where they are sampled and converted into discrete data points. At each time instant during the step t, the encryption algorithm in TSB is implemented as the following linear function: enc $(x t)=\alpha t \times x t+\beta t$, where $x t$ is the continuous analog signal magnitude, and $\alpha t$ and $\beta \mathrm{t}$ are calculated coefficient and offset. The coefficient and the offset values are calculated through a secure hash chain function, where the output of the hash function is fed as the input to the same function during next chain iteration. In particular, TSB makes use of the secure HMAC message authentication code implements a one-way hash function to transform an input value of any size to a 512-byte hashed value. To increase the security of the computation, the HMAC function also takes a secret key that is used during the hash value calculation, i.e., $\alpha \mathrm{t}+1=\operatorname{HMAC}(\mathrm{k}, \alpha \mathrm{t})$ and $\alpha 0=1$ where $\mathrm{k}$ is a symmetric key shared between the control center and the sensor. The offset value is similarly calculated. It is noteworthy that in TSB's current implementation, the time steps are intervals with equal lengths; however, to increase the unpredictability of the encryption coefficient and offset parameters and hence the cipher-text, one can calculate the interval lengths using another hash chain. Following the identical goal, it is also possible to change the phase of the $\mathrm{AC}$ signal to increase the entropy and amount of uncertainty in the encrypted signal. 
As both the TSB's substation-side encryption hardware and control center-side decryption agent are aware of the encryption function discussed above as well as the used secret key, the control center decrypts the received encrypted measurements using the inverse function, i.e., $\operatorname{dec}(x t)=(x t-\beta t) / \alpha t$, where the coefficient and the offset values are calculated similarly using the HMAC function. Note that the measurement values that are received by the control center are digitalized data points; however, TSB's decryption is still able to decrypt the values because the Analog-to-Digital operation within the PMU is a linear function and makes the inverse decryption procedure feasible.

It is noteworthy to discuss the key management scheme in TSB. The control center needs to setup a shared symmetric key with each sensor in the field (potentially at the sensor manufacturing time). The keys could be identical so that the control center stores a single key only; however, a more secure solution would be to establish different keys across sensors so that the whole framework is resilient against few sensor compromises by the attackers with physical access to the substations. Following the separate key scheme, the control center needs to process every communication with each sensor separately in order to calculate the subsequent decryption keys correctly.

\subsection{End Point Synchronization}

As discussed above, the control center and the current/voltage transformer sensors need to calculate the encryption and decryption parameters continuously. It is also crucial to complete these calculations and subsequent parameter updates on time so that both parties use the correct pair of values on both ends. In other words, TSB needs a synchronization mechanism to ensure that the parameter update times on both ends occur 
at exact same time points. Among the several possible solutions, we picked the global positioning system (GPS) clock due to their low cost, high accuracy, and ease of deployment. Both the control center and the sensor are equipped with the GPS clocks that provide them with exact time information. Using the installed sensors, both end-point parties update the cryptographic parameters periodically (for example every 30 seconds starting the midnight). Consequently, the decryption engine on the control center side will be guaranteed to be using the correct parameter values to decrypt the transferred sensor measurements.

We also designed a new protocol for cases where the GPS sensors are not available due to reasons such as weak signal coverage. In this scheme, to make sure that both parties are aware of the time step points where the receiving party needs to update its decryption parameters, TSB makes use of predefined signal patterns that are agreed upon before the system setup by both of the parties. In particular, the control center and the sensor agree upon the pattern of 10-number sequence that is rare during normal parameter updates according to the used hash function sequence. For instance, if the control center receives a sequence of values $(9,3,6,1,8,5,2,0,4,7)$ it identifies that the pattern is one of the patterns it had agreed with the sensor upon and updates its decryption parameters using the $\alpha \mathrm{t}+1=\operatorname{HMAC}(\mathrm{k}, \alpha \mathrm{t})$. The above-mentioned protocol is easier and cheaper to deploy compared to the former GPS-based algorithm; however, it is crucial to select the patterns carefully because the protocol will not function as it is supposed to be if the selected pattern happens to be one of the frequently occurring value sequences during the normal hash chain calculations. 
From protocol security analysis viewpoint, even if the attacker can guess an approximate estimate of the scaling coefficient during the sensor measurement transfer, he/she cannot infer the next scaling coefficient because of hashed values of two similar numbers are not (guaranteed to be) close to each other. For instance, if the attacker infers the scaling coefficient to be 3 that is close to its actual value $\alpha$ t $=2.99$, he/she will calculate the next scaling coefficient to be $\alpha \mathrm{t}+1=\operatorname{HMAC}(\mathrm{k}, 3)$ which is a completely different value than (often far from) the actual next coefficient $\alpha \mathrm{t}+1=\operatorname{HMAC}(\mathrm{k}, 2.99)$. This keeps the subsequent cryptographic parametric value update in TSB resilient against parameter sniffing intrusions.

\subsection{Implementations}

\subsubsection{Test Platform}

We developed TSB at the Smart Grid Test-bed laboratory at the department of Electrical and Computer Engineering, Florida International University (FIU) that was developed as an integrated hardware based AC/DC hybrid power system and explained in Chapter 2 of this dissertation.

The major elements for practical integration of our proposed method are the Smart Grid test-bed platform, GPS synchronization clock, encryption module, and commercial PMUs and PDCs (Figure 5.2).

\subsubsection{Hardware specifications}

This system emulates a small-scale power system, with 3 phases at $208 \mathrm{v}$ nominal voltage and $60 \mathrm{~Hz}$ nominal frequency but capable of operation under different voltages and frequencies. The test setup was designed to operate for maximum of 15 amps in 
steady-state operation mode in transmission lines and maximum voltage of $130 \mathrm{v}$ (phase to ground). This hardware platform uses PTs and CTs to scale down the power side signals into accessible level, which can be utilized for measurement and evaluation purposes. In particular, PT ratio in our system is 20 and $\mathrm{CT}$ ratio is 3 . It means that every $20 \mathrm{v}$ in power side converts to $1-\mathrm{v}$ and every 3 Amps will be represented by $1-\mathrm{v}$ at the point of measurement. This was selected based on acceptable input range for data acquisition that is $+/-10 v$. Detailed information is available Chapter 2 . For this study, several types of hardware have been employed and each will be explained in the following sections.

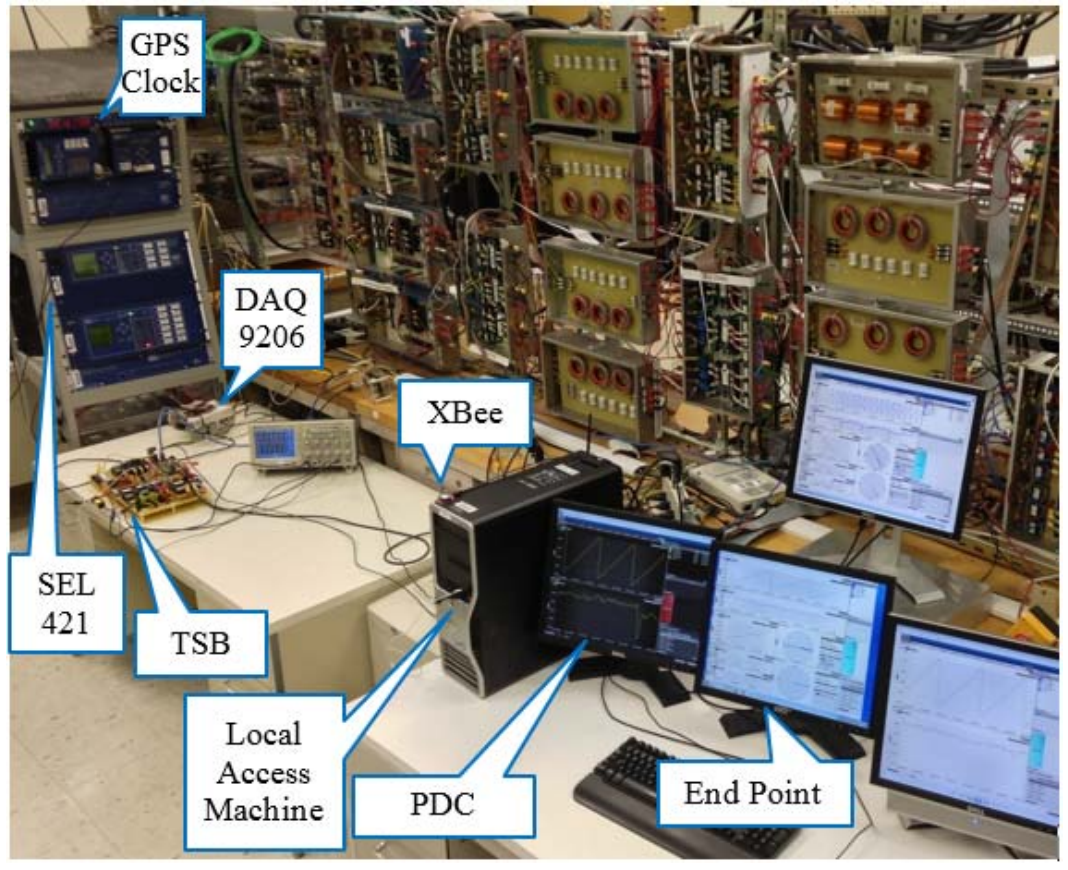

Figure 5.2 TSB experimental setup 


\subsubsection{GPS Clock}

In order to have synchronization at the sensor and the control center side, TSB uses GPS clock. It receives the UTC time information from 24 GPS satellites around the earth and prepares the time information in IRIG-B Format. IRIG (Inter-Range Instrumentation Group) is an accurate and standard format and described in IRIG standard 200.04 [102]. It has different formats and IRIG-B is the one selected for power system applications. It has 100 bits that bring in the information of year, day of year, hour, minute and second with an accuracy of sub-microsecond. This signal can be modulated or unmodulated. The modulated signal is an amplitude modulated format with a sinusoidal carrier, and unmodulated signal is a TTL format (DC-pulse width with variable duty cycles) which has no carrier. Since the unmodulated signal is feasible, it was selected for our case.

The experimental setup utilized an industrial GPS clock SEL-2407. For this clock, a GPS antenna is installed on the roof and the clock will provide online IRIG-B signal for our application. In case connection to the satellite fails, the clock has the capability to use an offline backup time source, which is an internal high precision clock with quite good accuracy and negligible error.

\subsubsection{Encryption module}

The encryption module converts power system measurements (in this case $3 \varnothing$ voltages and currents) into an analog time synchronized encrypted signal based on the hash value.

Figure 5.3reveals the details of trusted environment, flow of signal from power side to end point and communications involved in this method. 
Figure 5.4 shows the developed prototype circuit. This circuit is located at the place of measurements (for example inside a substation). It is designed to autonomously encrypt $3 \emptyset$ voltage and current measurements based on proposed algorithm along with considerations to communicate with the processing unit through serial RS232 port.

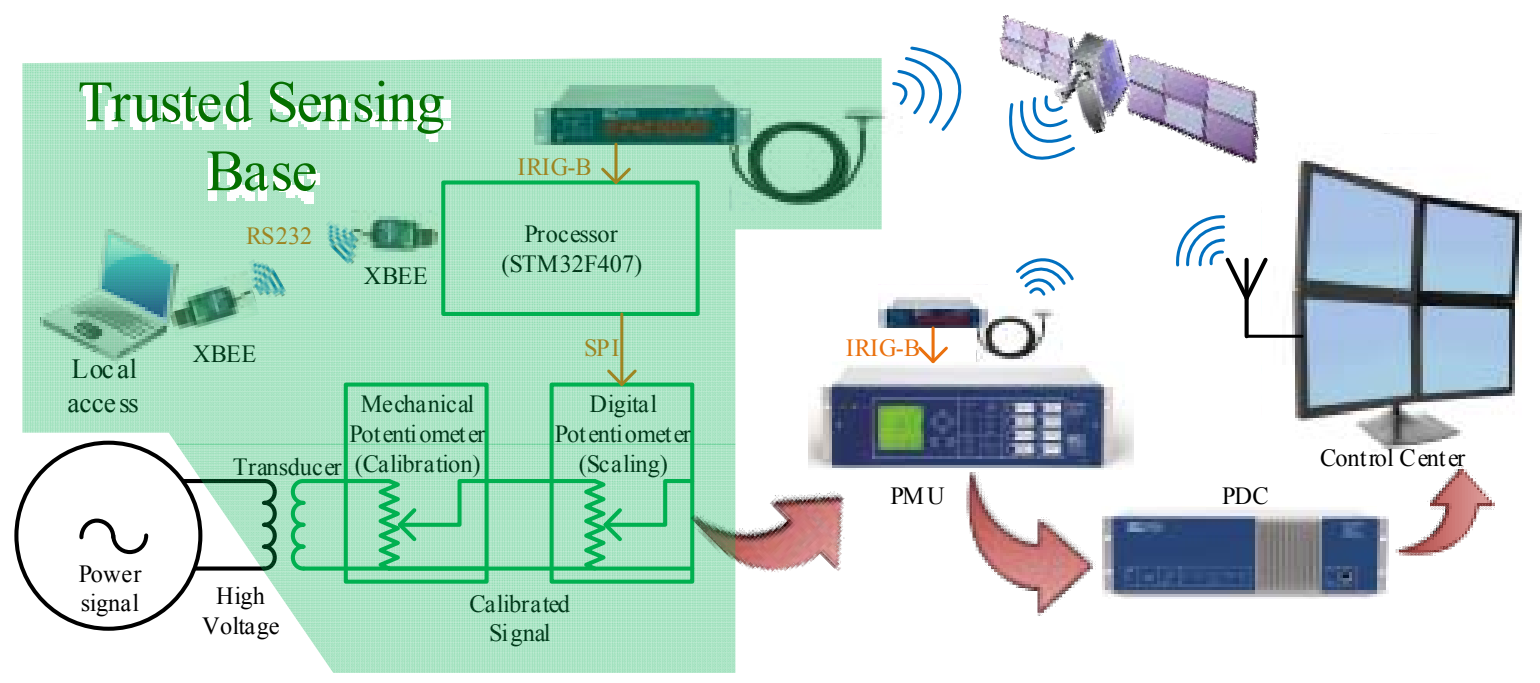

Figure 5.3 TSB environment and flow of signals 


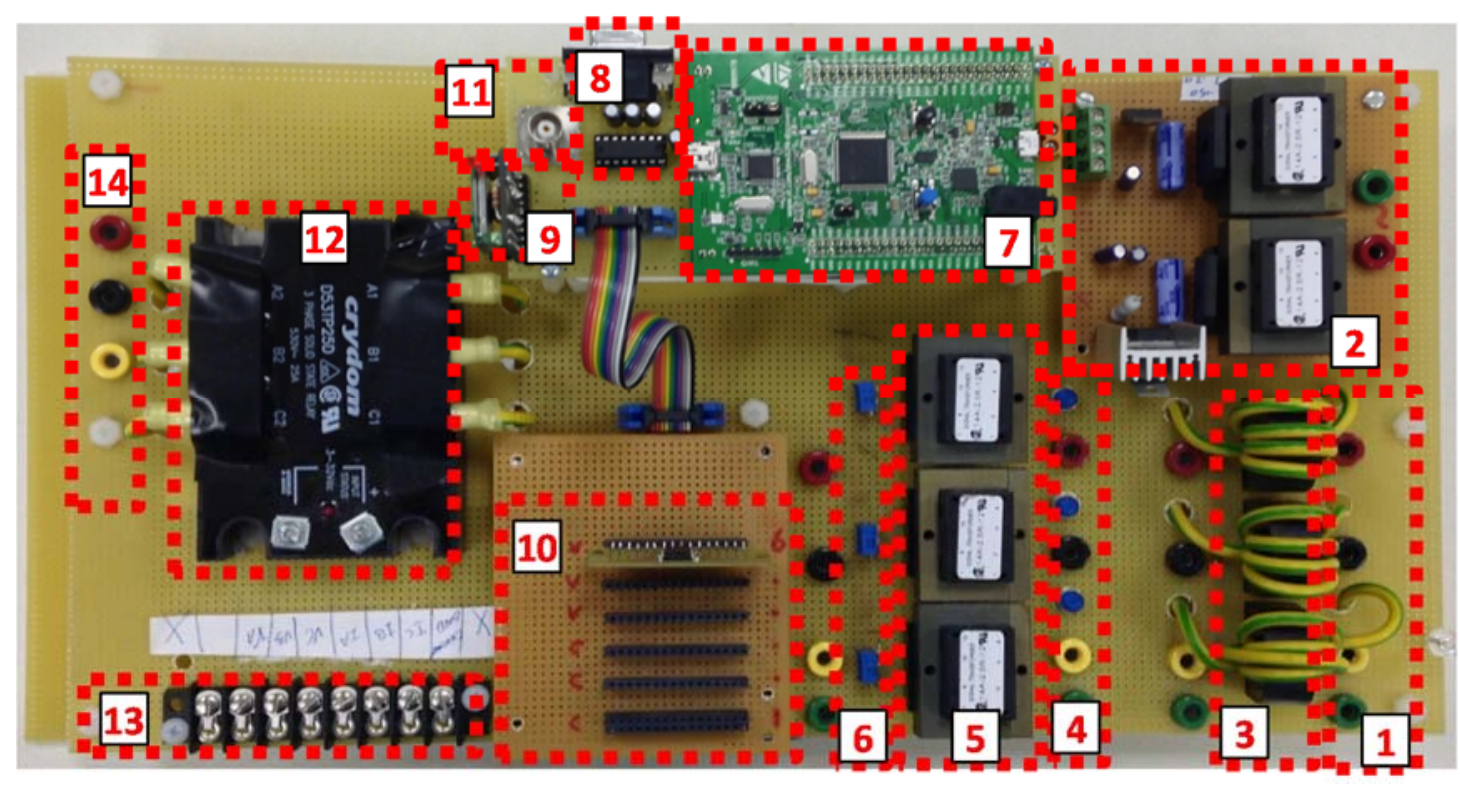

Figure 5.4 TSB prototype

Components of TSB prototype in Figure 4 are as the following:

1) $3 \emptyset$ input power terminal,

2) Local Power supply,

3) $3 \emptyset$ Current transformers,

4) 3 Power connection indicators,

5) $3 \emptyset$ Voltage transformers,

6) Mechanical potentiometers,

7) Embedded processing unit,

8) Wired serial port,

9) Wireless (XBEE) serial port,

10) 6 slots for Digital potentiometers $(3 \varnothing * 2)$,

11) IRIG-B Input,

12) Solid state switch (Breaker), 
13) Encrypted signal terminal (output),

14) $3 \emptyset$ output power terminal

The processor which was used in the embedded processing unit is a STM32F407 on a Discovery Board. It is programed by Matlab Simulink using Waijung library, working at $168 \mathrm{MHz}$ clock speed and utilizing I2C, SPI, UART, IRQ, DIO peripherals available on this chip. A combination of utilized library and Discovery board enhances flexibility of programming and testing without worries about compatibility in a friendly environment. It is recommended just for the prototyping stage and optimum programming can be done in future stages.

The processing and communication directions in the embedded system are demonstrated in Figure 5.5. The system should be capable to receive time from a realtime GPS clock through DIO peripheral, send/receive time to/from external time source, communicate with local operator, and send scale commands to a Digital Potentiometer in high speed.

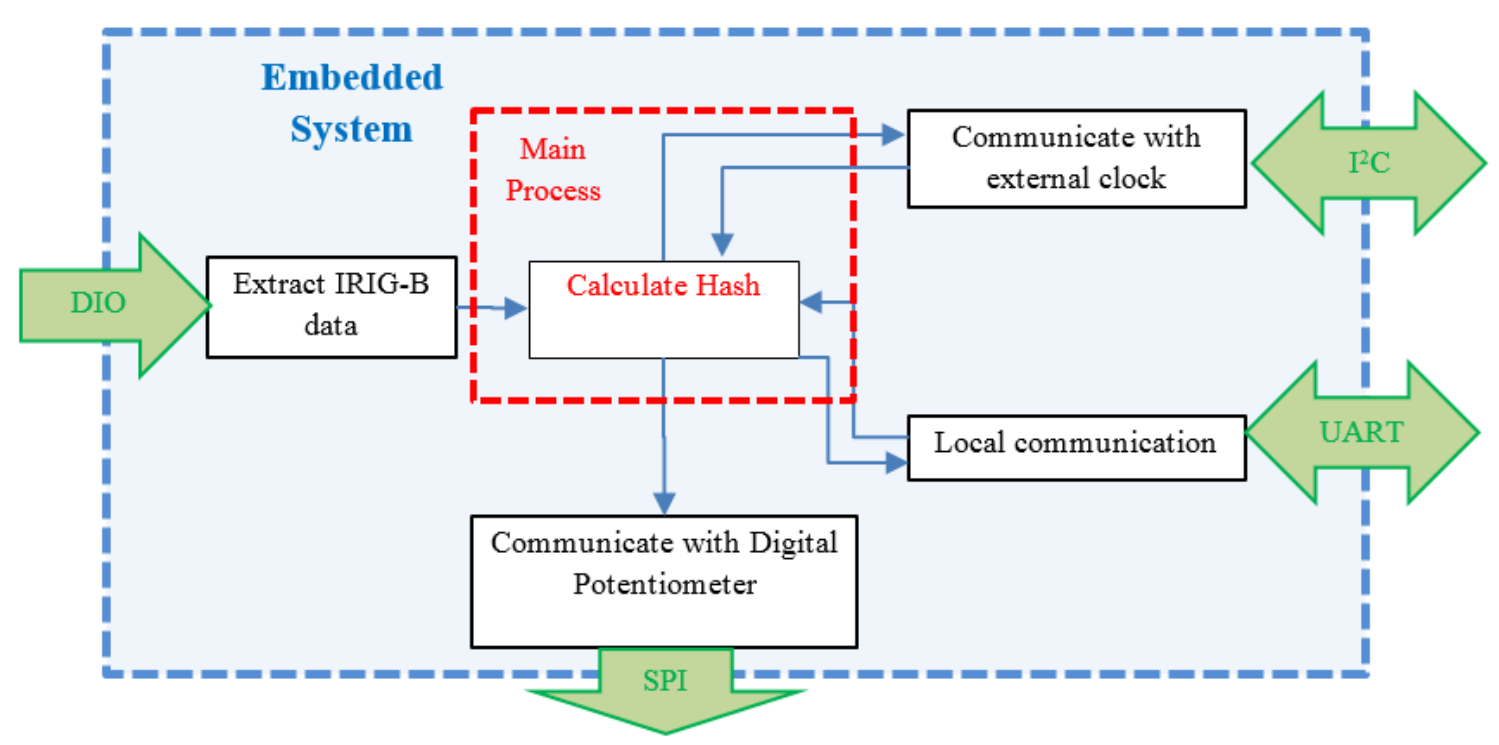

Figure 5.5 Association of communication channels with main process unit 


\subsubsection{External Clock}

In order to keep the process synchronized and be able to operate without online/offline GPS clock, an external time source was experimentally tested for the system. For this purpose, a DS3231 chip is deployed using I2C communication protocol. Although it is extremely accurate time source, it is not good enough for this application. Experimental tests showed that the clock has a $\pm 3 \mu \mathrm{s} / \mathrm{s}$ drift, and the cumulative error during long period of operation will result in considerably out-of-synch sensor and control center ends. This chip has an alarm capability, so it can trigger the encryption device (in our case STM32 processor) to apply the new encryption (new scale). Even by neglecting the time drift of the DS3231, another drawback is that it is not able to synchronize and harmonize the triggering instant with any external reference. In other words, the alarm is capable of sending a trigger every second, but this trigger has an out of control constant offset time shift less than 1 second which makes it worthless for our application.

\subsubsection{Calibration}

As mentioned in 5.5.2, CTs and PTs are converting the power side signal to lower scale to be accessible for measurements purposes and data acquisition. Since these transducers (current and voltage transformers) are not ideal and cannot perfectly transform the voltage level, a calibration is required to manage this issue. The major error is because of incorrect gain (Transformer ratio), we use a mechanical potentiometer to correct the ratio with negligible error. 


\subsubsection{Scaling procedure}

The proposed method is working autonomously, changing the scale of the analog signal synchronously at both sensor side and end side. The time information plus a key (any user defined number, string, password) in a HMAC function creates a hash and this associates to a scale that will be applied to the digital potentiometer.

\subsubsection{Digital Potentiometer}

The digital potentiometer is the component that is used to apply the concept of TSB to change the analog signal within $0-100 \%$ range. In an ideal case, it needs to be linear (without deviating the signal), fast (can be applied instantly), accurate (low error from expected value), continuously scaling (any scale) and reliable. But in reality, physical systems have some limitations that will affect the quality of encrypted signal and finally performance of proposed method for actual applications.

In a practical test, three different digital potentiometers have been tested including MCP4161, MAX5439 and AD7376. They are all using Serial Peripheral Interface (SPI) protocol for communication. SPI has the capability to work in a high frequency $(\mathrm{MHz})$ clock and minimum required frame bits.

MCP4161 is only capable of working in positive voltages, eventually it is not applicable when the signal has a negative portion (in our particular case sinusoidal waveform). MAX5439 required 9 bits SPI and it is not compatible with our processor hardware. A solution can be using bit-banging technique, but it is not recommended since it uses long processing time and finally reduces the quality of TSB.

AD7376 is compatible with the processor and supports positive and negative ranges. All digital potentiometers usually have $2 \mathrm{~N}$ discrete steps and consequently the 
digitizing error is $1 / 2^{\wedge}(\mathrm{N}+1)$. These steps are evenly distributed between $+/$-Vref. In our case, the Vref is $+/-15 \mathrm{v}$ and the local power supply module prepares those in addition to +5 and $+3.3 \mathrm{v}$ that are applicable for components used in the circuit. AD7376 has $27=128$ steps and $50 \mathrm{k} \Omega$ full range resistance.

The maximum SPI clock is $4 \mathrm{MHz}$ but it can be over clocked to $5 \mathrm{MHz}$ without loss of communication. Required time for data transfer between processor and digital potentiometer was evaluated in Section VII.

\subsubsection{Sampling frequency}

One of the main advantages of TSB is that it does not change the contents of the analog signal. So any kind of transient phenomenon will stay unaffected and can be extracted at the next level of measurement by the analog to digital converters. Data acquisitions can use their original sampling frequency with no need for reconsideration of requirements in the new scheme.

\subsection{Evaluations}

An implemented TSB prototype was evaluated in several features including two major aspects time requirements and accuracy analysis.

\subsubsection{Time Analysis}

Our main evaluation focus is on delays and time shifts.

\subsubsection{Settling Time, DC Test}

TSB has to be applied instantly so will not affect the measuring signal during the transient of scaling procedure. 
Figure 5.6 shows the settling time of scaled signal from the instantaneous moment when digital potentiometer starts to change the scale until it reaches the steady state. In order to investigate this property, the input signal is selected to be a $+5 \mathrm{vdc}$ and the scale is changing from $110 / 28$ to $10 / 128$. Acquired conversion time is about $3 \mu$ s and it shows that the transient is fast enough for real-time applications.

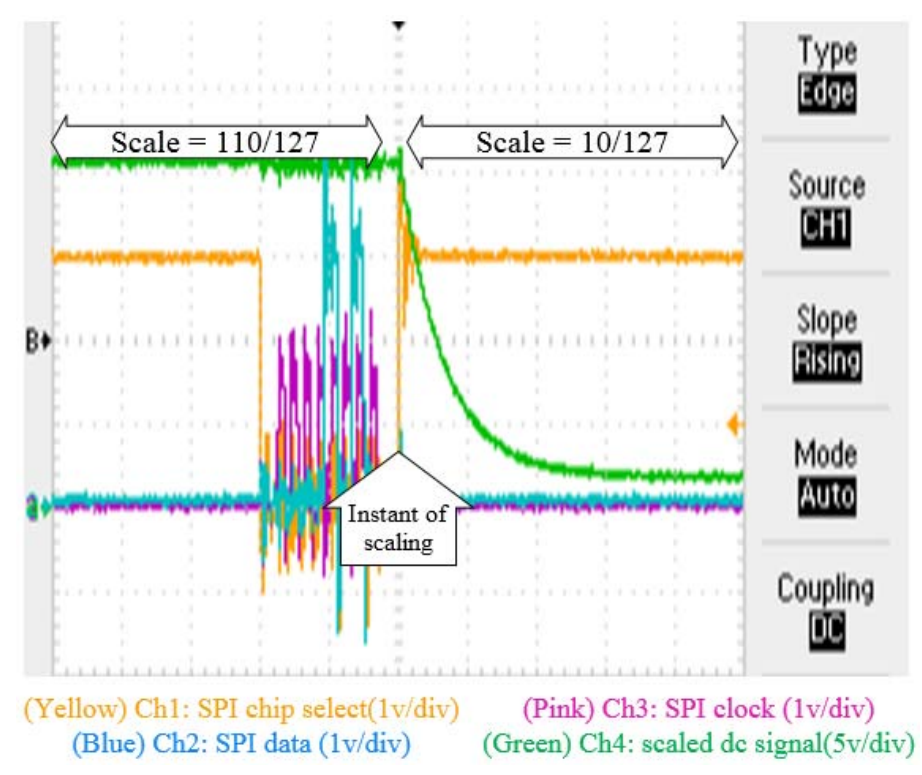

Figure 5.6 DC test, settling time

\subsubsection{UART command and delay}

Figure 5.7 and Figure 5.8 are simultaneously captured and they are showing the scaling instant changing from step $0 \%$ to $100 \%$ when the user defined change is applied through UART channel set to 115200 Baud rate and 8-N-1. 


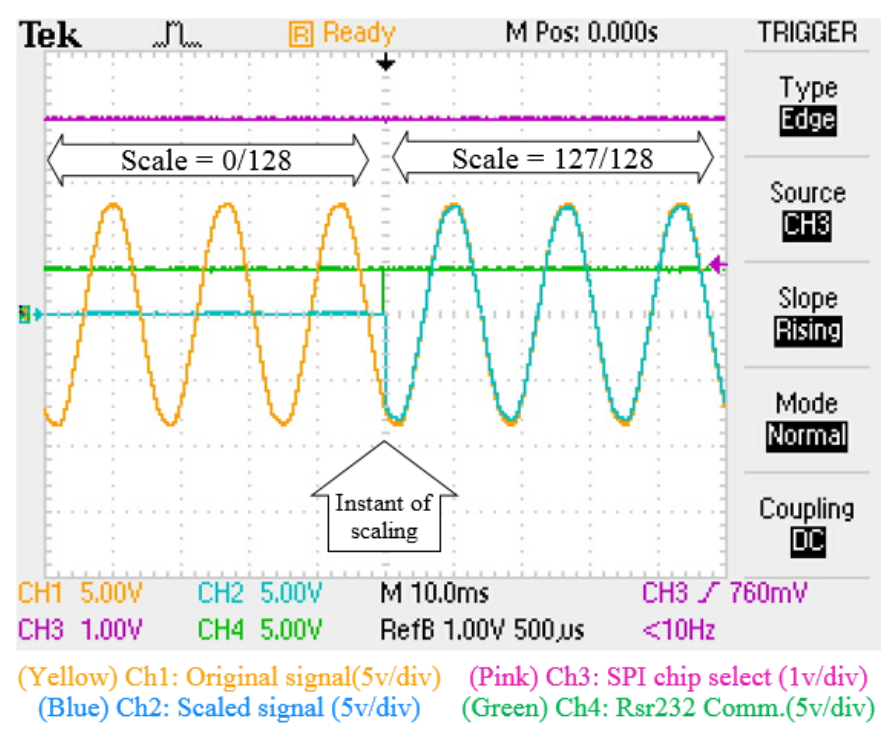

Figure 5.7 AC test, communication delay for RS232 command

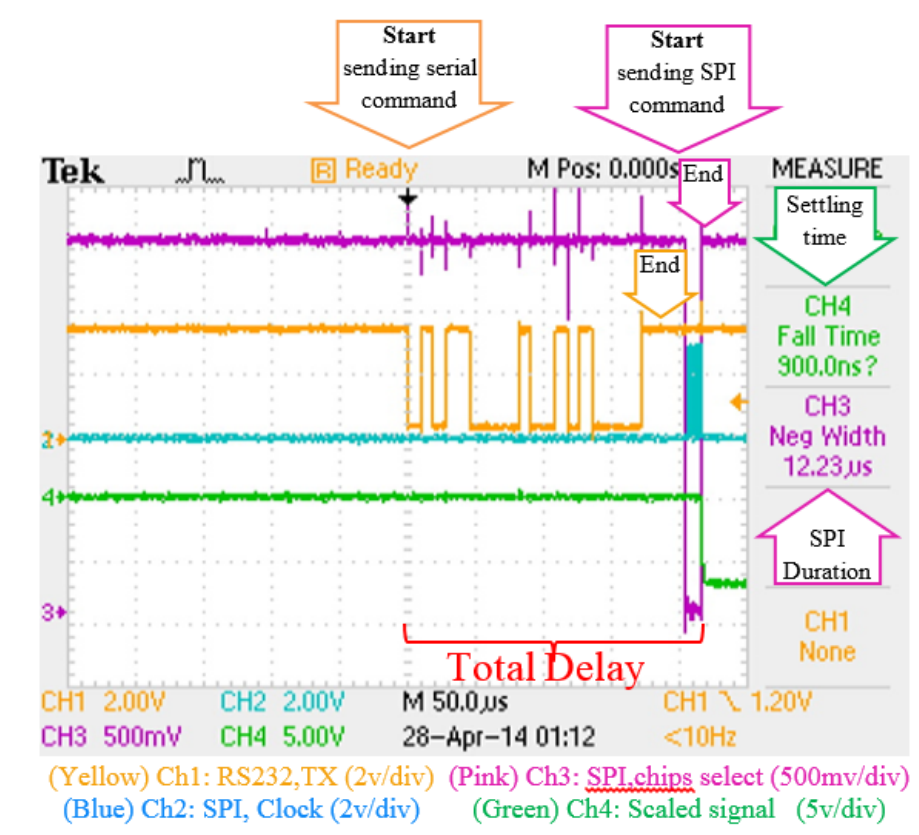

Figure 5.8 AC test, communication delay for RS232 command

In this case, the SPI Clock is set to $0.4 \mathrm{MHz}$. The total delay is more than $200 \mu \mathrm{s}$ that is analogous to sinusoidal waveform period. 


\subsubsection{GPS Autonomous Command and Delay}

TSB is proposed to work autonomously at the sensor side and end point. Figure 9 and Figure 10 are captured simultaneously to show the timing performance of TSB using autonomous trigger by GPS clock. In these cases, scale is changing from 90/128 to 0/128 and at the specific instant when UTC changes.

Figure 5.9 demonstrates the automated scaling when it is synchronized with an IRIG-B signal provided by GPS clock. In this case there is no delay related to serial communication to/from local computer/operator, and this makes the automated system almost real time, and fast enough for real time application.

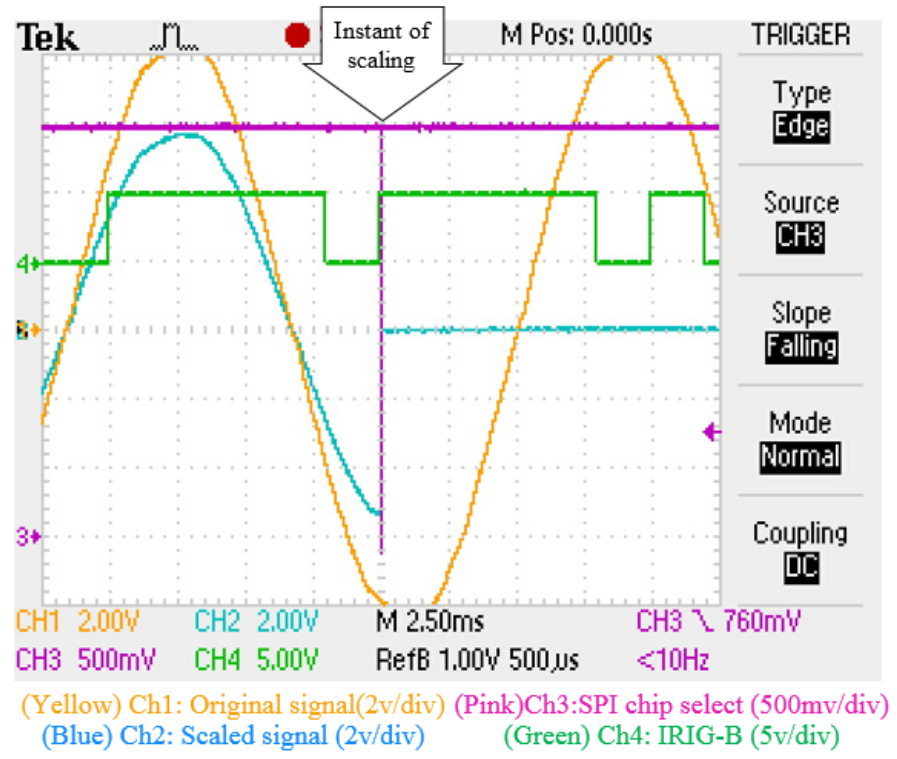

Figure 5.9 Autonomous scaling of signal triggered by IRIG-B

Figure 5.10 provides a better vision for timing performance of TSB in autonomous operation. The SPI clock is increased to $5 \mathrm{MHz}$. As it can be seen, Scaling can be provided in less than $15 \mu$ s delay, which includes processing, SPI data transfer and conversion inside digital potentiometer. 


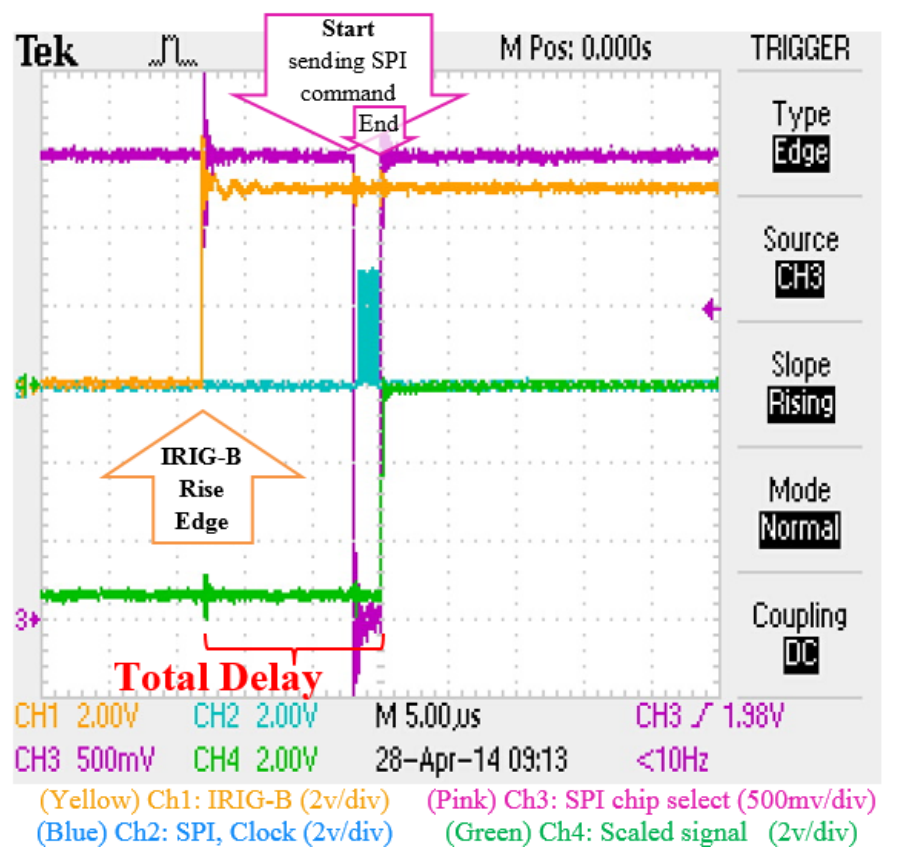

Figure 5.10 Total delay in autonomous operation triggered by IRIG-B

\subsubsection{Accuracy Analysis}

The used scaling module (digital potentiometer) needs to have accurate and linear functionality. Several properties of this module have been evaluated in the following segments.

\subsubsection{Zero Percent scale test}

In some applications, such as authenticating purposes, there might be a need to ultimately suppress the measurement signals. So a $0 \%$ scale will be applied to digital potentiometers and in idyllic form expected signal should have 0 RMS value. This test was executed to a digital potentiometer and RMS measurements.

Figure 5.11shows the suppressed signal in addition to peak-to-peak, and DC offset. 


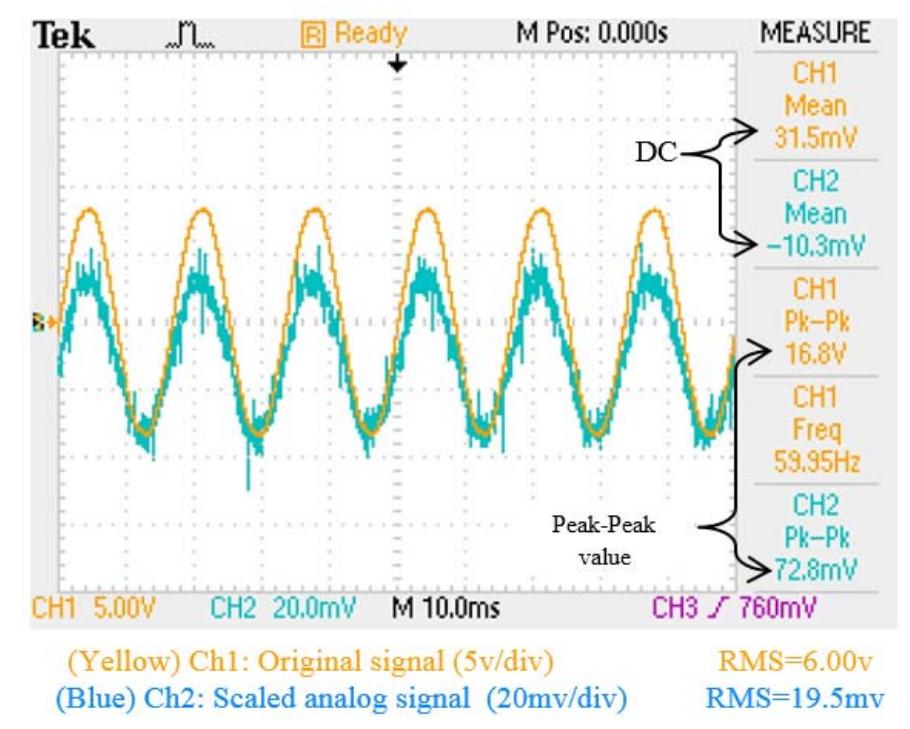

Figure $5.110 \%$ Scaled signal in comparison to original signal

\subsubsection{2. $100 \%$ Scale test}

In contrast to section 5.6.1.1, a $100 \%$ scale should provide the complete original analog signal in ideal case.

Figure 5.12 shows the original signal, scaled signal and their difference.

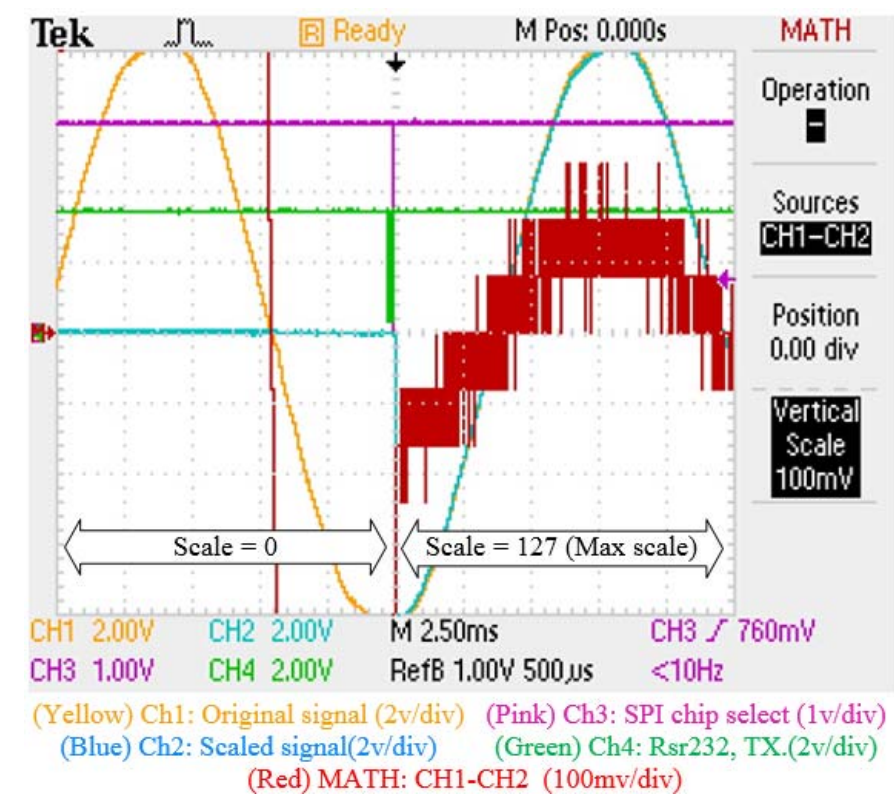

Figure $5.12100 \%$ Scaled signal in comparison to original signal and showing their differences 
After changing the scale to $\sim 100 \%$ (which is $127 / 128$ ), the red waveforms shows the difference which supposed to be zero in ideal situation. Experimental results show that the maximum error for $100 \%$ scale is about $600 \mathrm{mv} / 16.66 \mathrm{v} \simeq 3.5 \%$.

\subsubsection{Overall Scale Error}

For all scaling steps (from step 0 to 127) the dc scaled signals have been compared with expected values and the results have been represented in Figure 5.13. In this experiment, the input signal was $+4.95 \mathrm{vdc}$ and the outputs are covering all steps (27) of digital potentiometer. The resultant errors have been calculated for each scale and they are illustrated in the same Figure 5.13.

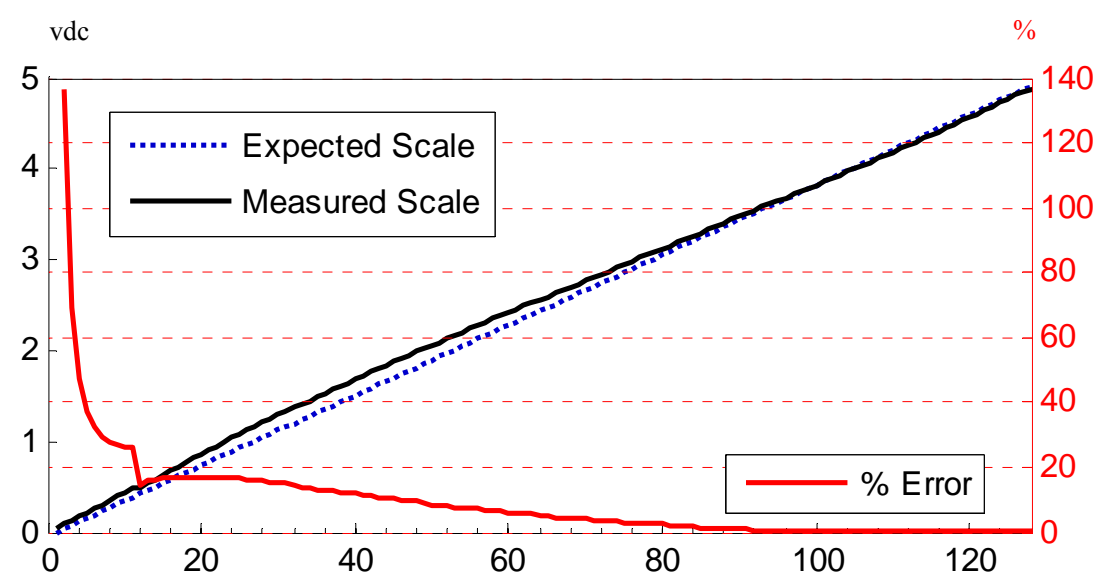

Figure 5.13128 Scales, expected, measured and \% error values

\subsubsection{Sampling with NI DAQ and Waveform Reconstruction}

The final encrypted signal was measured through the NI DAQ9206 module and the waveform was reconstructed and compared with the original signal and final comparison depicted in Figure 5.14. The waveform is captured in 6-kHz of sampling for a window of 0.5 seconds. In this experiment, the scale is changing every $0.05 \mathrm{~s}$. 


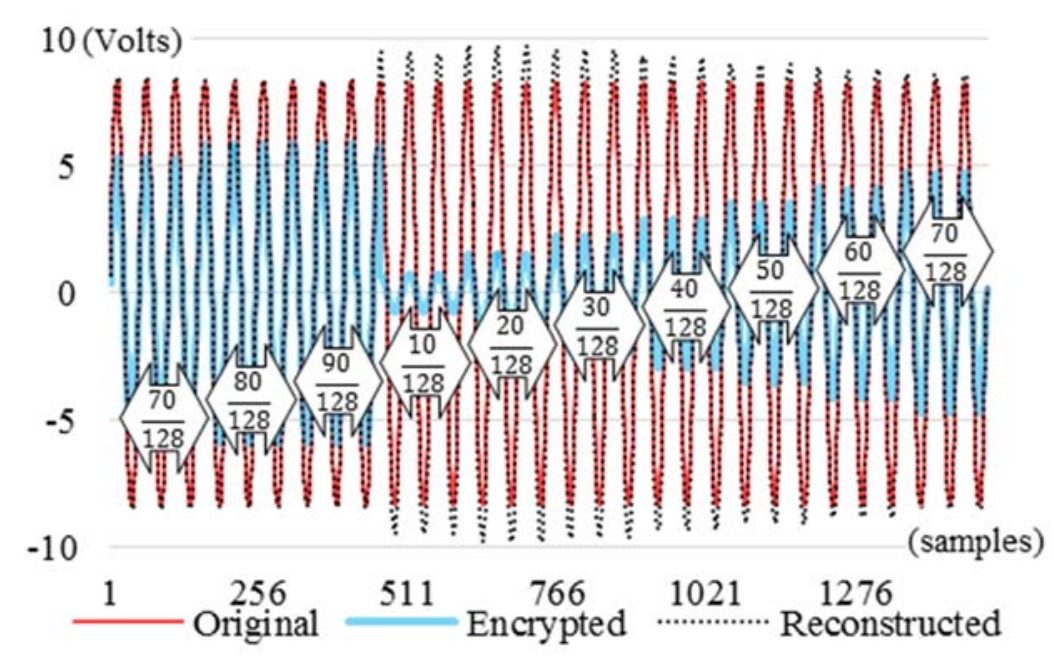

Figure 5.14 Comparison of original, encrypted and reconstructed waveforms

As expected from the results of Section 5.6.2.3, a big percentage of error in smaller scales will distort the amplitude at the point of reconstruction. Consequently, it is recommended to use bigger scales or apply a compensation coefficient to each scale, in order to remove this error.

\subsubsection{PMU Results and Reconstruction}

In a practical implementation, the encrypted analog waveform was measured by PMU SEL-421 and the amplitude of the original signal and encrypted signal have been compared for a period of 30 seconds. In this period, PMU provides the amplitude and phase information through synchrophasor protocol and send it to PDC SEL-5073 by rate of 5 message per second. Figure 5.15 shows that encryption method successfully maintains the signal information, but it is vague for any attacker who decides to compromise the sensors. 


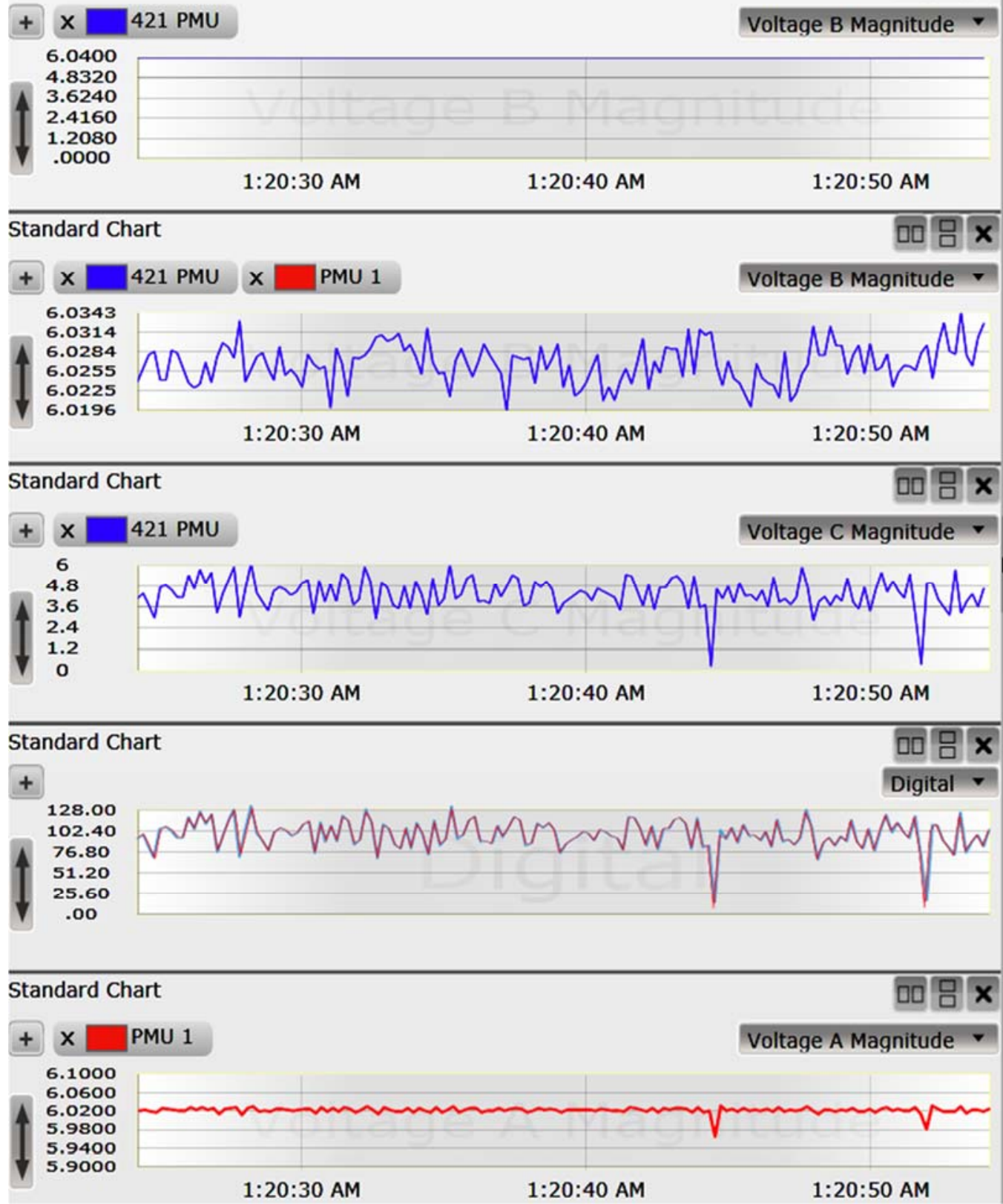

Figure 5.15 PMU SEL-421 readings: (a) Original RMS readings (b) Original RMS readings (c)Encrypted

RMS readings (d) Applied and actual scales (e) Reconstructed RMS values

\subsection{Conclusion}

In this chapter, we present TSB, a trusted sensing base to protect power grid critical infrastructures against false data injection attacks. In particular, TSB deploys a 
GPS-based analog data encryption within sensors at the data acquisition point, i.e., the first point where data encryption could be implemented. Consequently, the attackers capable of control over vulnerable phasor measurement units or A-to-D devices remain unable to manipulate acquired sensor data maliciously. Our extensive implementation and experimental results in real-world power system test-bed shows that TSB could efficiently reduce the size of the infrastructural trusted computing base through reducing the number of assets in power grid that need to be trusted for the whole infrastructure to give correct functionality. 


\section{DEVELOPMENT OF HARDWARE IN THE LOOP TECHNIQUES FOR MICROGRID CONNECTIVITY}

\subsection{Introduction}

Microgrids are going to be the basis of future active distribution networks. They are capable of integrating Distributed Energy Resources (DERs), provided that they are coordinated and operated efficiently. There are several international microgrid research implementations, which are ongoing and their R\&D objectives are:

- Study the operation of microgrids to decrease carbon emissions and to increase penetration of renewable energy resources

- Study the operation of microgrids in parallel with the main grid and stand-alone as islanded grids

- Ensuring the efficient, reliable, and economic operation and management of microgrids by defining and developing control strategies

- Assuring the safety, fault detection, separation, and islanded operation through defining appropriate protection and grounding policies

- Identification and development of required communication infrastructure and protocols

- Demonstrating and simulation of microgrids in laboratory scale [103]

\subsection{Benefits of DC Micro Grid Deployment}

Most of the electronic devices, such as computers, routers, lights, TVs, communication systems are supplied by the DC power. DC environment is a more 
convenient way to deliver power to such loads in order to assure reliability and scalability. One of the main benefits of only DC networks is no need of AC to DC converters (rectifiers); therefore they have significant efficiency improvements. Current power supply efficiencies in the market for AC systems is about 60 to $85 \%$, but in the DC systems, it is about 85 to $90 \%$.

Another advantage of a DC network is integration of storage systems and batteries. Grid scale storage systems can improve the stability of the DC grid, as well as the AC grid. It may prevent the main grid from blackouts. Although grid scale battery storage systems are not practically functional, utilization of distributed batteries are more applicable. The summation of these distributed batteries is equivalent to a large bulk energy storage, except for the fact that they may have more losses, because of far distance of installed batteries on the field. These losses include the conversion losses in the inverters, transmission and distribution losses, and finally, the rectifier losses at the load place.

Another advantage of a DC microgrid is the enhancement of the integration of renewable energy resources, such as PV, fuel cell and wind turbines. Additionally, DC networks can improve the efficiency of the Plug-in Hybrid Electric Vehicles (PHEVs) and Electric Vehicles (EV) and simplifies their integration into the hybrid grid. They have no phase specification, so can be easily connected to the DC batteries and this can be extended for vehicle to grid or vehicle to vehicle applications in a large scale system.

By considering the DC network capabilities to reserve the energy, they can improve the reliability of power systems. It can increase the burdens of AC systems, 
when excessive amount of power is available, and restore it for the time when there is a need for power.

\subsection{Integration of the Hybrid Grid by Development of AC/DC Microgrids}

In order to integrate the renewable energy resources, several microgrids was considered and designed in the Smart Grid Testbed. In the following section, several case studies for interoperability of Hybrid power system will be presented.

\subsubsection{AC Grid Operation}

The designed platform, which is explained in chapter 7, and the corresponding Human Machine Interface in NI-LabVIEW, which is explained in chapter 3, will provide the environment to operate an emulated a real-time $\mathrm{AC}$ power system. The main generator VI provides the generator power and their loading in order to share the generation level, when one of them faces an overload situation. G1 (the main generator) works as the slack generator and others (G2, G3, G4) will serve as PV units and they contribute to balance the demand according to their communication and control command during the real-time operation.

\subsubsection{Building the Initial Power Grid}

The procedure for making an interconnected AC grid starts with the slack bus and a local load. Since there is no AC grid at the beginning, slack bus does not require any synchronization process and will directly power up the initial grid. This initial grid can be extended to adjacent transmission lines, load buses and generator buses, but not connected to other generators unless certain conditions are satisfied. Since other generators are designed to be working as PV buses (operating in torque control mode), 
they cannot deliver the synchronization requirements in order to be connected to the grid. That is because they are controlled through a torque control command from the control center, and the torque control mode has a resolution of $1 \%$ in each step, which is proportional to a $50 \mathrm{Watt}$ for a $5-\mathrm{kW}$ generation station, consequently, proper synchronization principles may not be obtained, and hence, the process requires a better control technique.

\subsubsection{Synchronization and Adding More Generation Stations}

This problem can be solved through a dynamic load, which works as a local load at the generator terminal to slow down the speed of rotating shaft of the generator and accordingly was named "Dynamic Brake". It is introduced in chapter 11 of this dissertation.

After applying the proper torque command and the dynamic brake controller concept on each of the generators, the synchronization procedure will lead to a successful and safe connection to the main grid. This process is depicted in Figure 6.1 and Figure 6.2, and Figure 6.3 and Figure 6.4 for the system represented in Figure 6.5. 


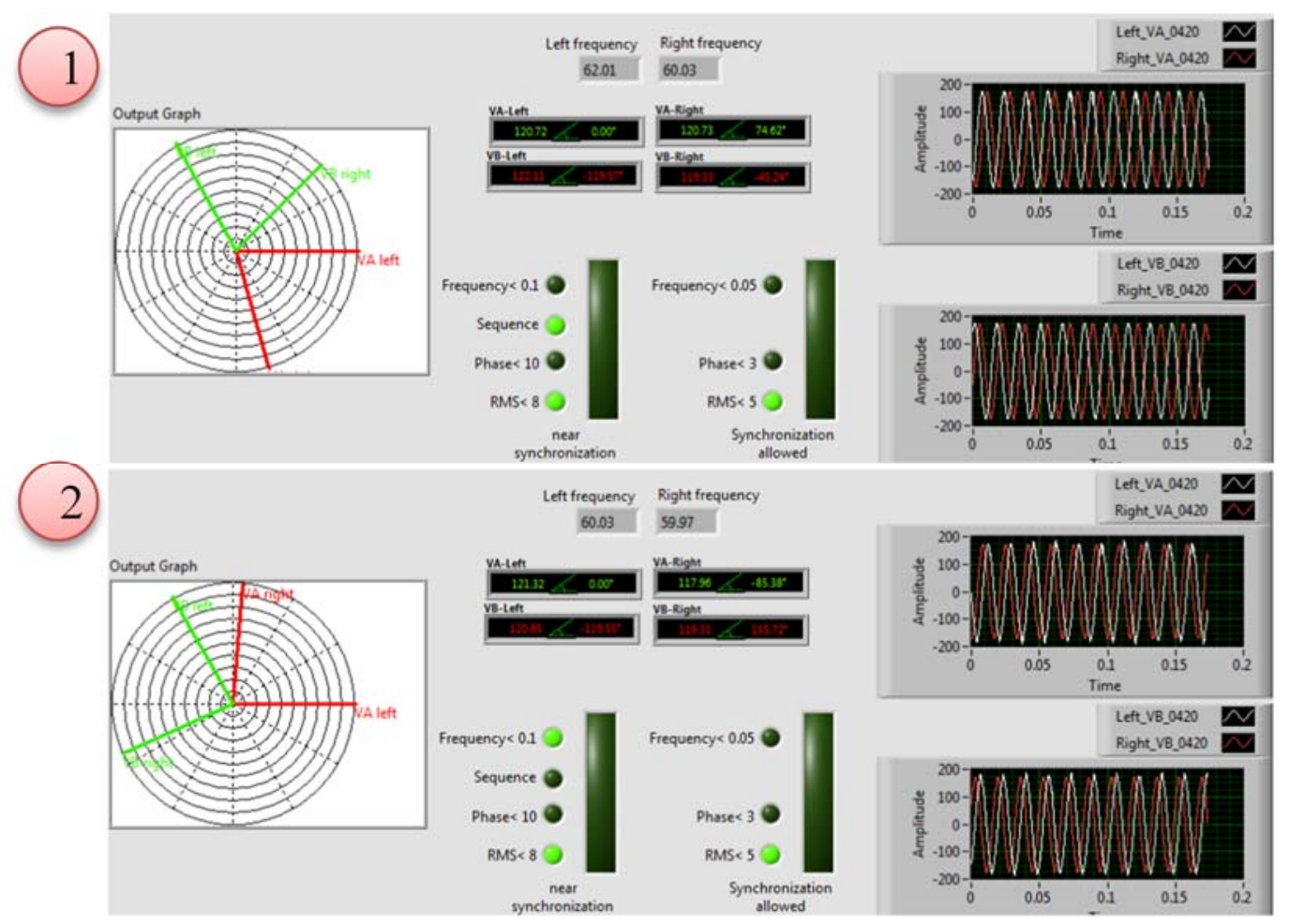

Figure 6.1 Step by step process of synchronization, 1) before, 2) at the beginning of the synchronization of G2 to bus0380 through synchronizer0420 


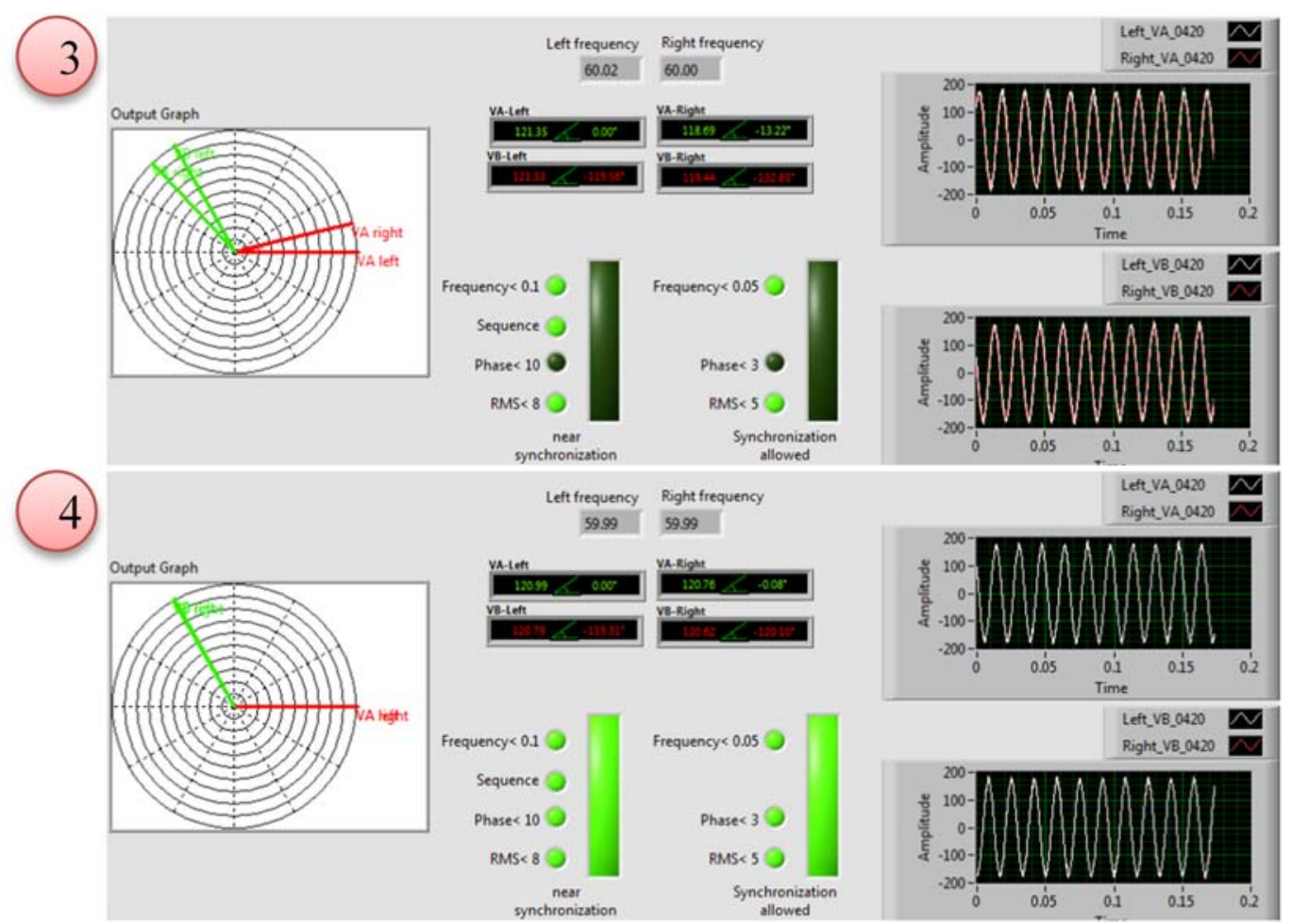

Figure 6.2 Step by step process of synchronization, 3) during, and 4) after synchronization of G2 to bus0380 through synchronizer0420

This process can be executed on all the other generation units, one after the other.
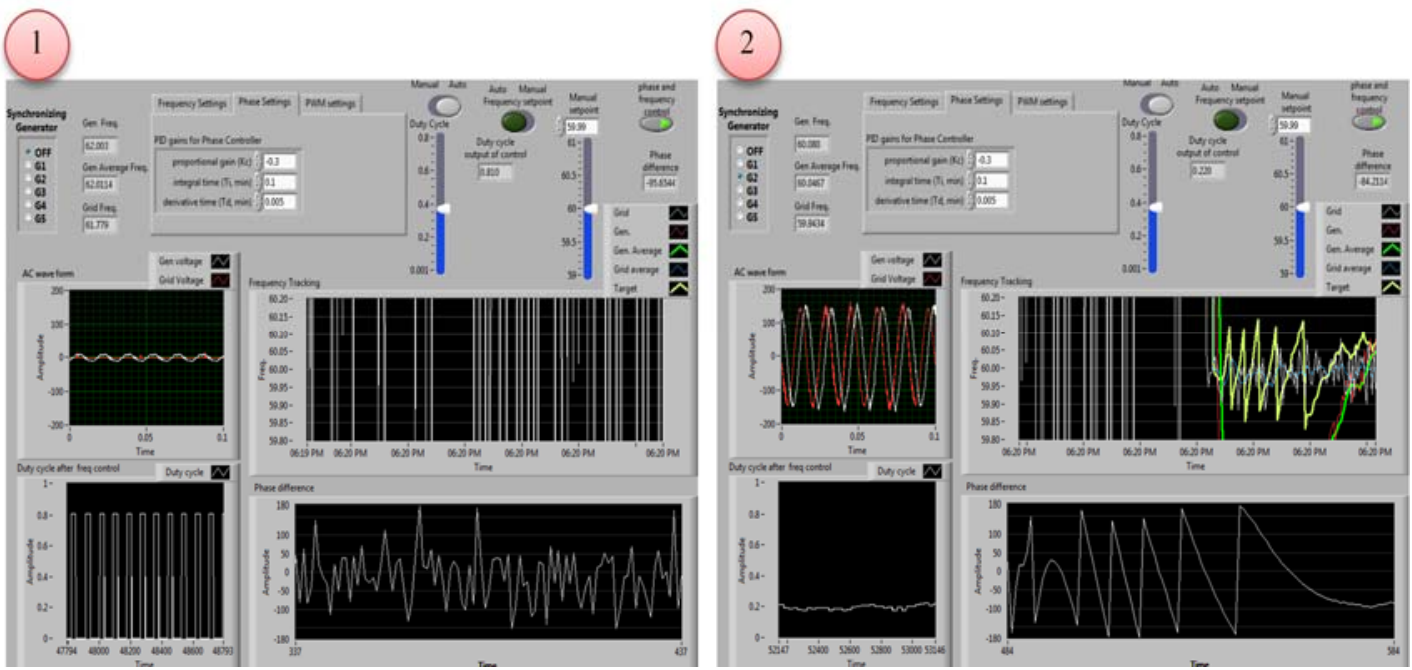

Figure 6.3 Integration of dynamic brake, 1) before, 2) at the beginning of the synchronization process of G2 to bus0380 through synchronizer0420 


\section{3}

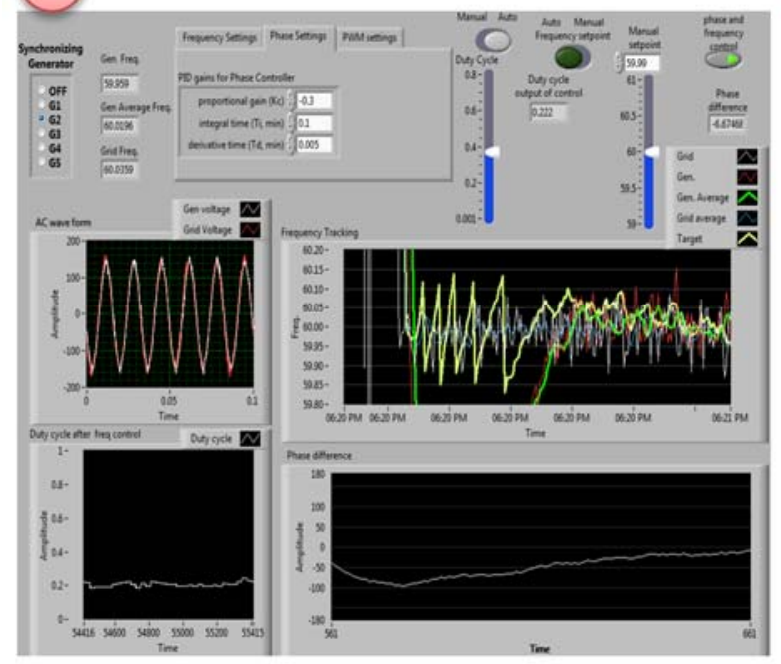

$(4$

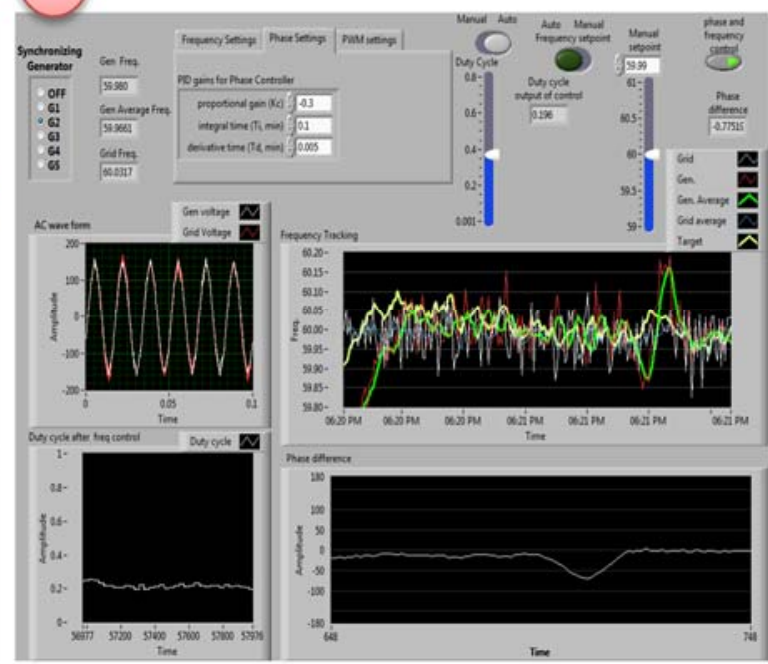

Figure 6.4 Integration of dynamic brake, 3) during, and 4) after synchronization process of G2 to bus0380 through synchronizer0420

\subsubsection{Load Balancing by the Slack Generator}

With no change in the generated power of other units, the slack generator is responsible to maintain the grid frequency at $60 \mathrm{~Hz}$ (or $50 \mathrm{~Hz}$, based on the frequency that

we would like to have in our system). Any load change will stimulate the controller of slack generator to react and keep the load (+loss) and generation balance in order to have persistent steady state frequency.

Figure 6.6 shows the changes of reactive power of generators $(\mathrm{G} 1,2)$ for 650VAR of inductive load steps at Inductive Load 1 (IL1) for the grid represented in Figure 6.5.

By changing the active and reactive loads of the system in Figure 6.8, the experimental tests show their operational status of the generator (G1) and the response to each load step changes as expected slack bus. 


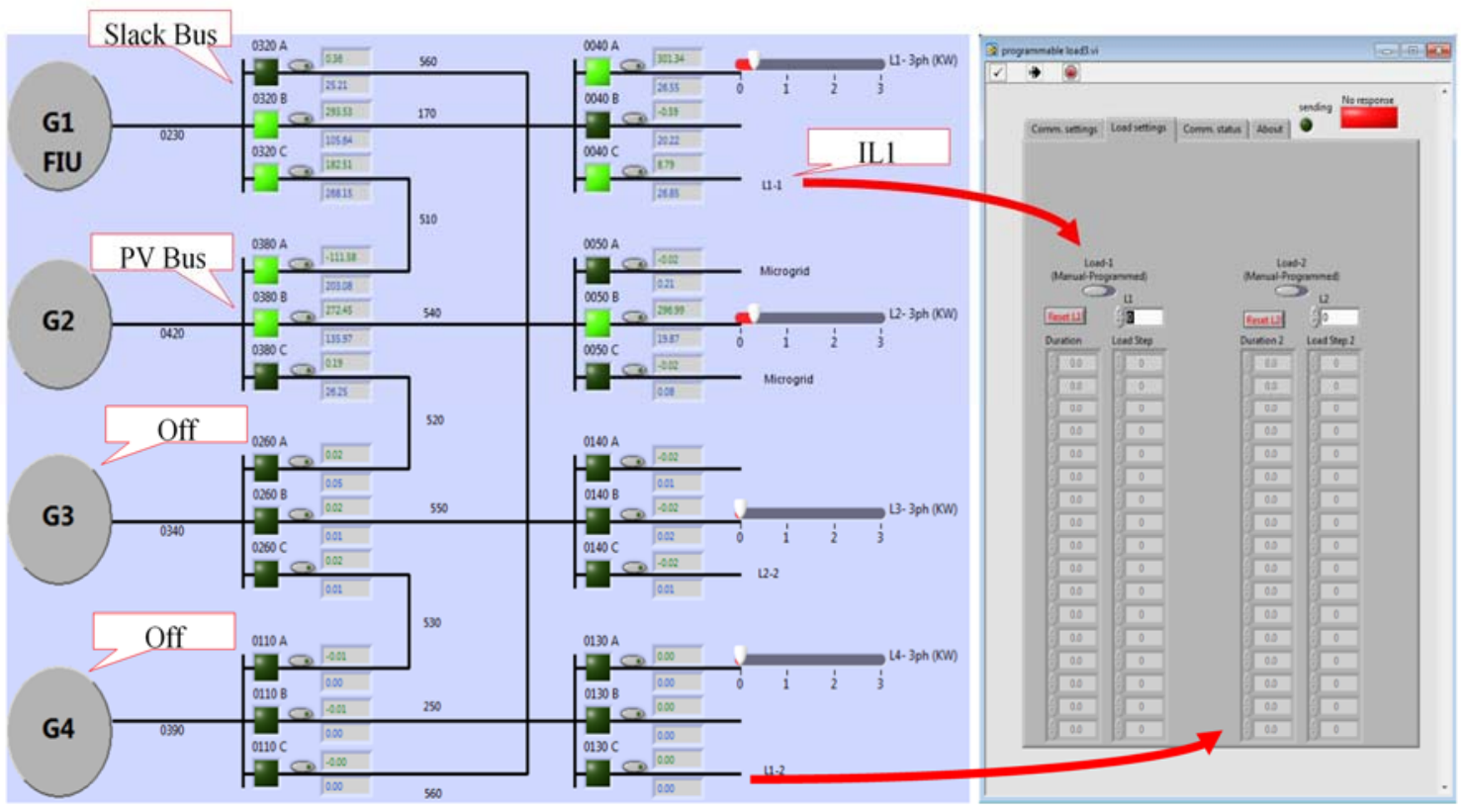

Figure 6.5 System configuration in reactive load response study and corresponding inductive load controllers

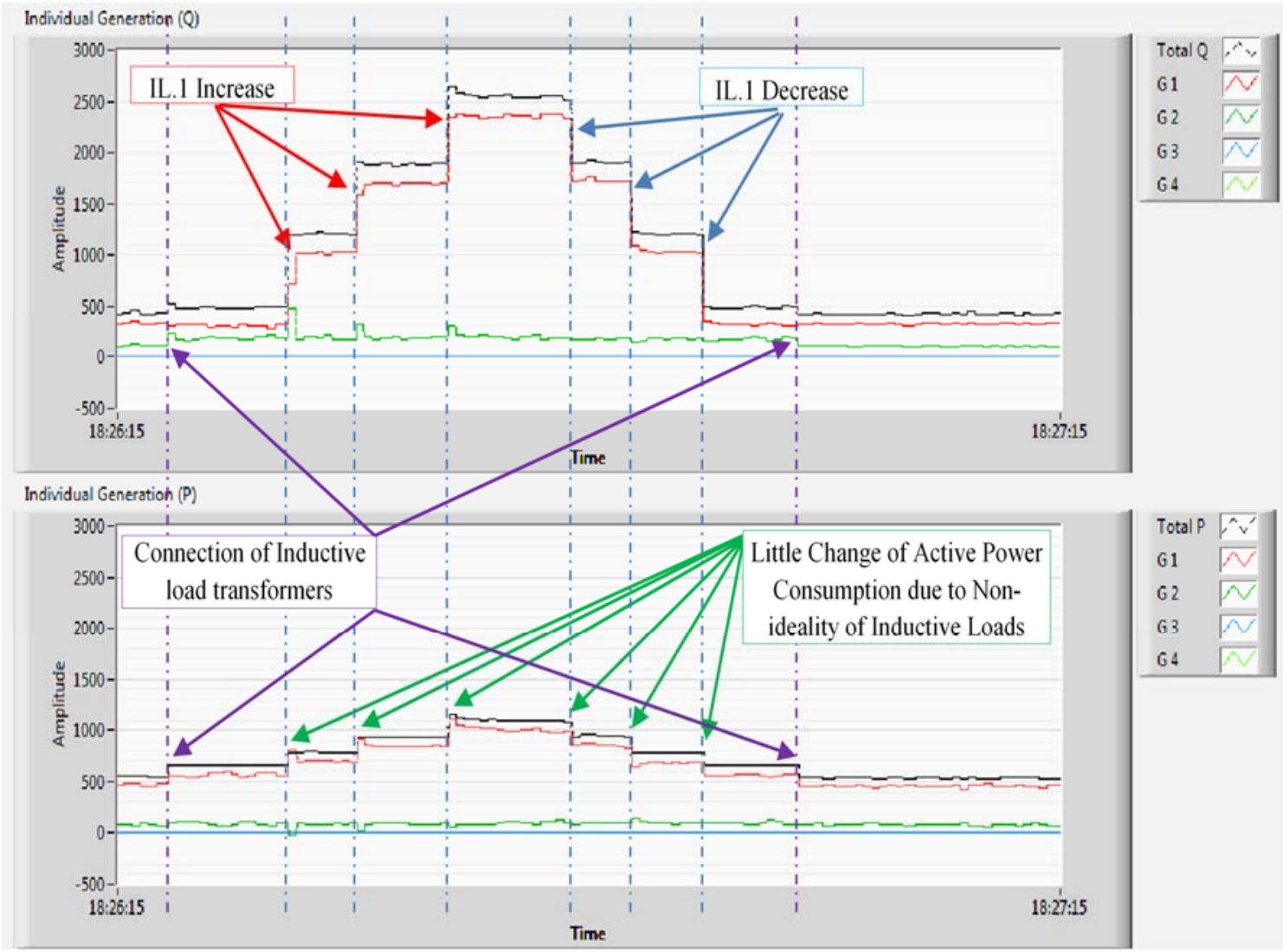

Figure 6.6 Respond of G1/G2 on reactive power change at IL1 (Period of 1 min.) (System of Figure 5.3) 


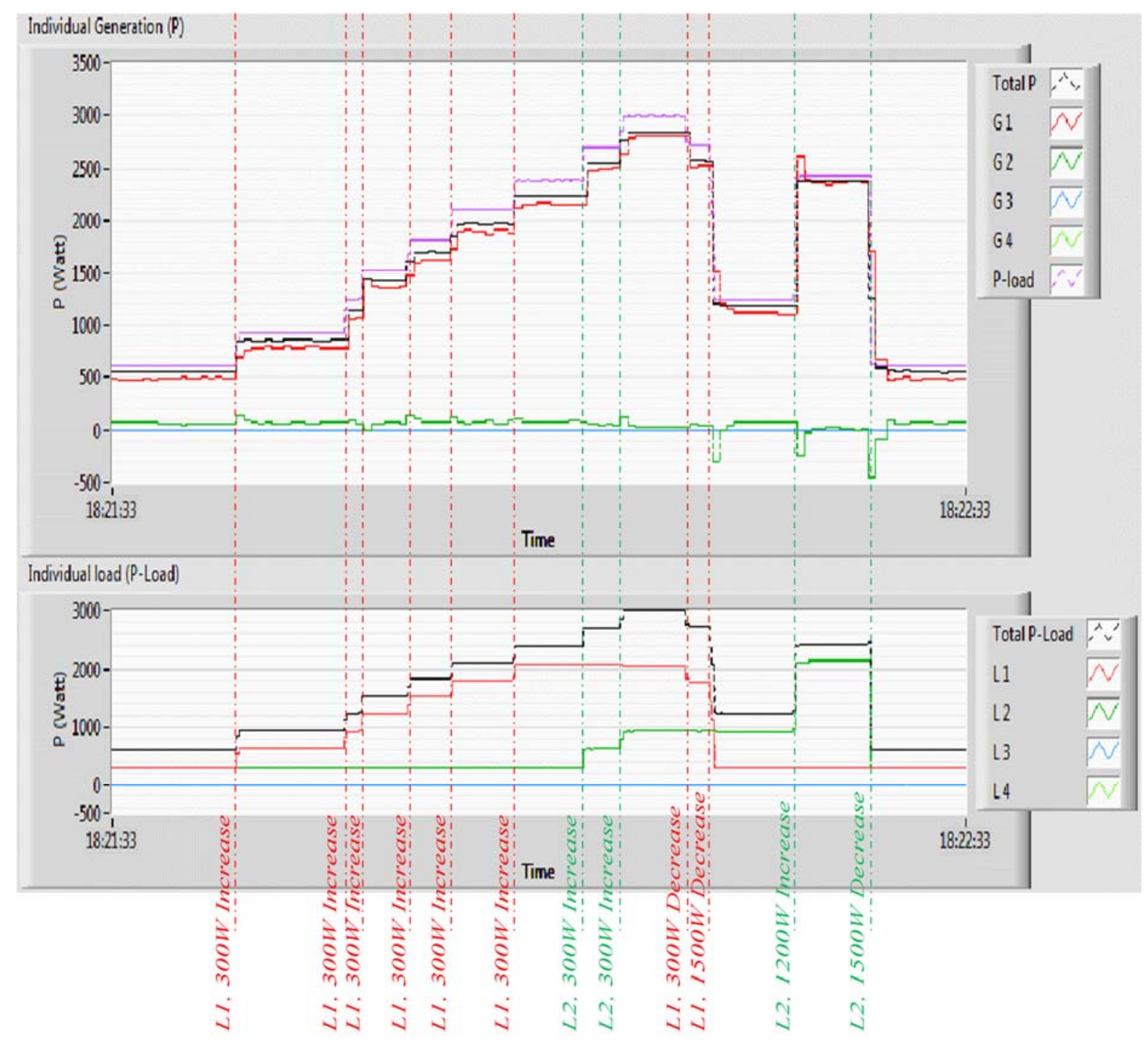

Figure 6.7 Response of G1 and G2 to change of L1 and L2 (Resistive loads) (Presented in Figure 5.3)

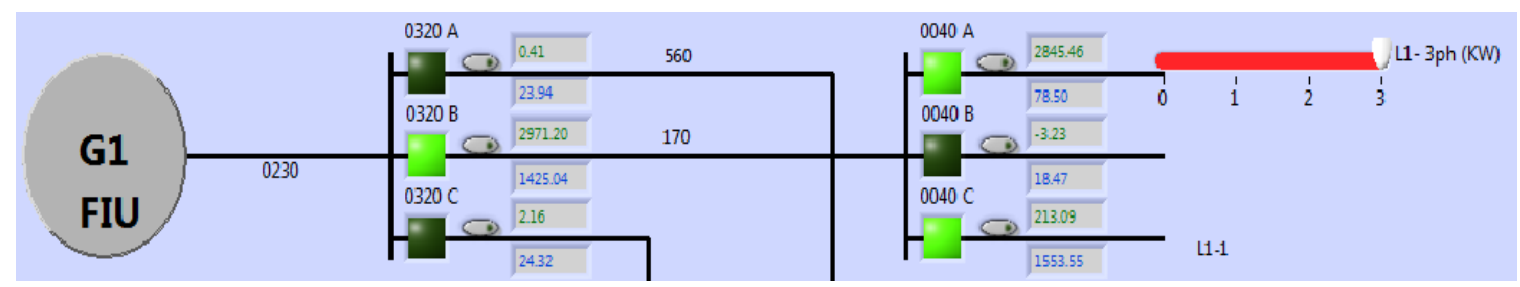

Figure 6.8 The SCADA representation of the system for inductive and resistive load change. 


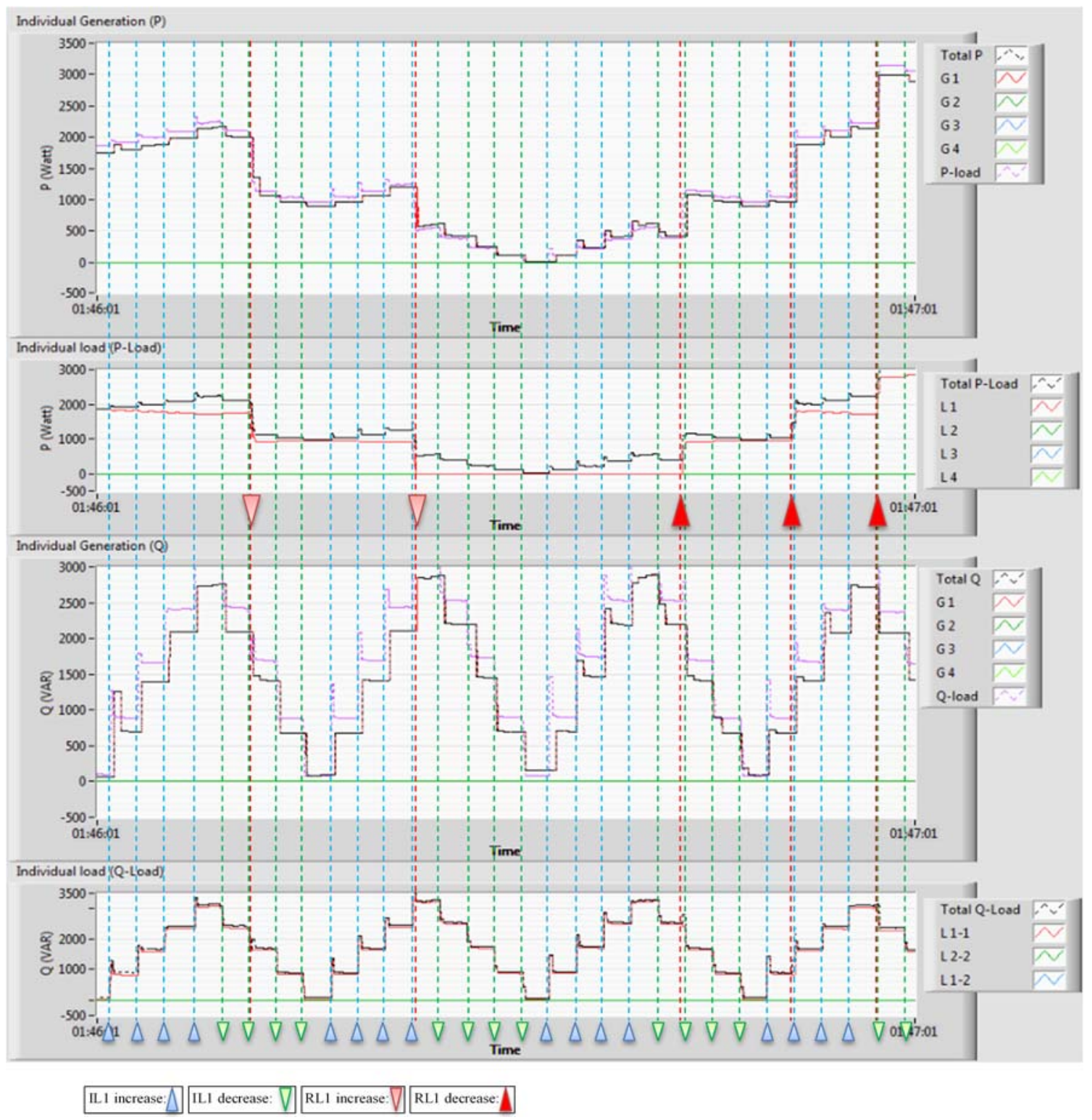

Figure 6.9 Inductive and resistive load changes and their impact on the generator

\subsubsection{Contribution of PV Buses in Generation}

PV buses are defined as constant voltage, and constant active power nodes in the power system. As it is explained in section 7.2, the generation station are working in PV mode using AVR and Torque-controlled mode of prime movers. 
An experiment was defined to verify the PV characteristic of generation stations, and it includes a system with one slack and one PV unit (as in Figure 6.10).

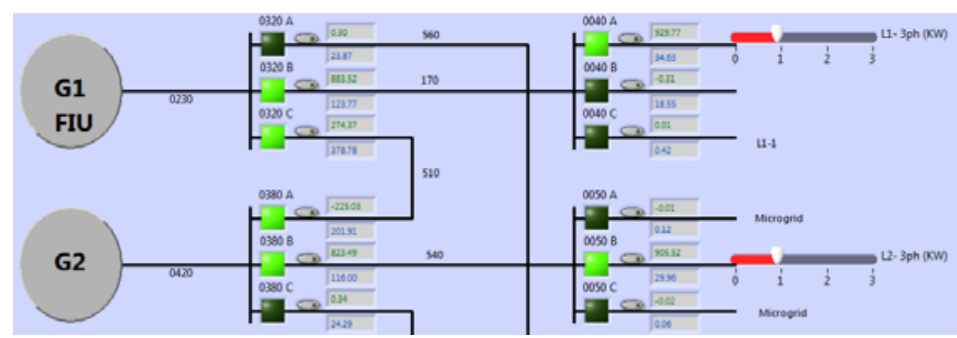

Figure 6.10 The SCADA representation of the system with PV and slack bus

G2 is set to work as $33 \%$ torque, equivalent to $567 \mathrm{~W}$ at the generator terminal and G1 is controlling the frequency to be $60 \mathrm{~Hz}$.

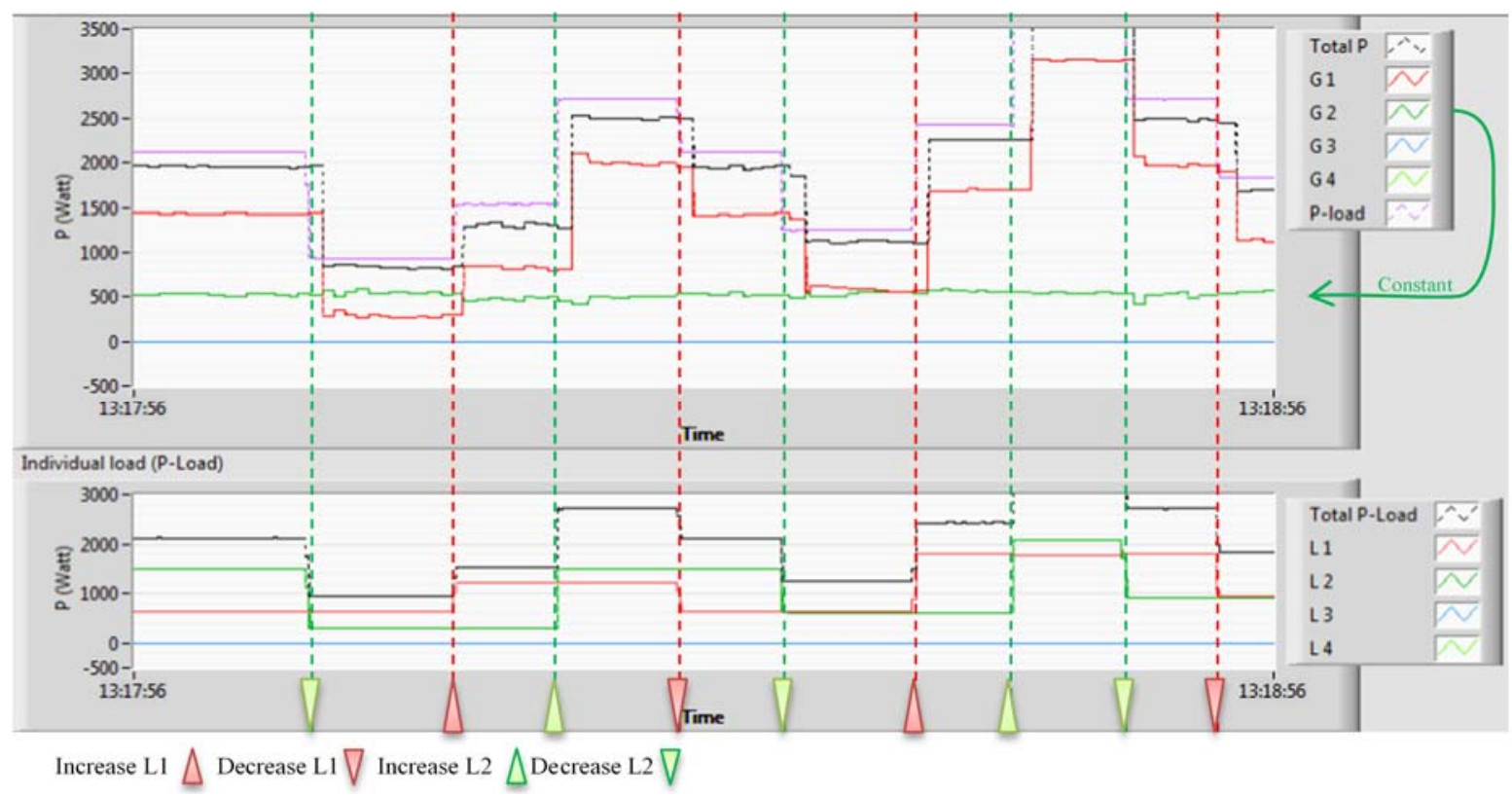

Figure 6.11 Power sharing between AC generation stations

As expected and can be seen in Figure 6.11, increase and decrease of loads will affect the G1 output power, and the total generations is equal to the total loads (+losses). Moreover, the Generation of G2 is constant and that proves the correct operation of it as a PV unit. 


\subsubsection{Hybrid Operation of Photo Voltaic Micro Grid for Sharing Power with AC Grid}

As mentioned in Chapter 8, The DC microgrid is connected to the AC grid through the bidirectional converter (rectifier/inverter). It can inject specific amount of active or reactive power to the point of common connection (PCC).

In this experimental example a load-generation balance study involving both $\mathrm{AC}$ and DC resources will be studied. The Microgrid connectivity point and the AC system architecture is presented in Figure 6.12. It has 8 buses, with the initial loading of about 300W in each load bus ("0040A","0050B","0140B","0130A"), and a variable inductive pulsed load of 780/1560VAR, with a period of 8 seconds, located at feeder " $0040 C$ ". The system is supplied from the infinite bus "FIU" with the fixed frequency of almost $60 \mathrm{~Hz}$.

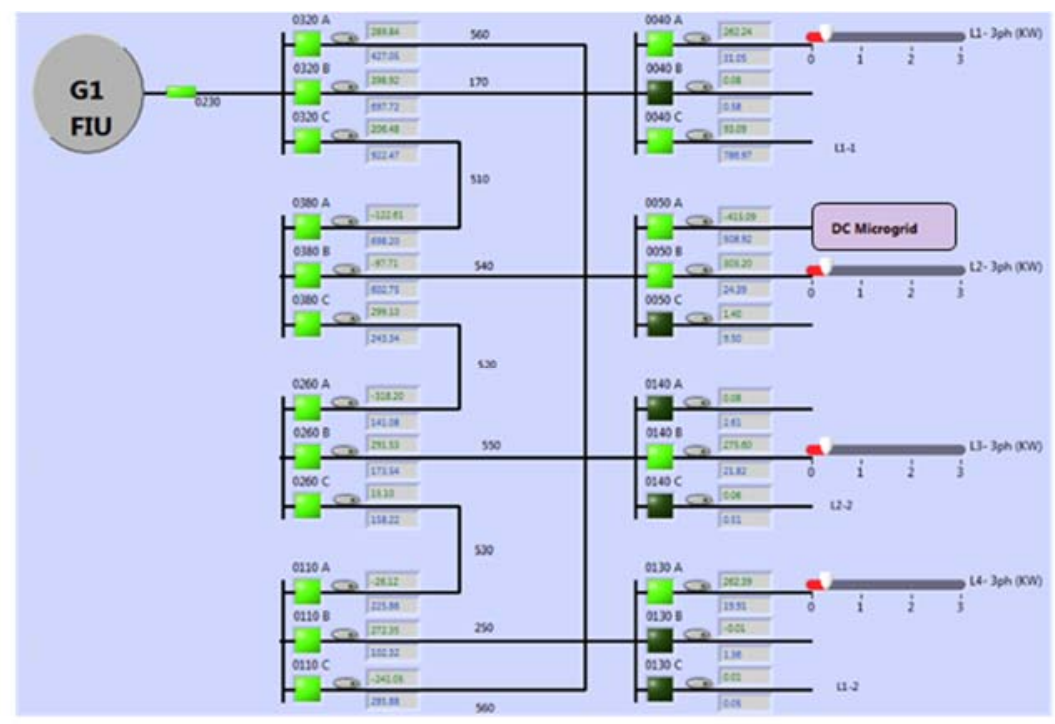

Figure 6.12 Architecture of the system under study

The scenario of applied events and the response of the system in addition to frequency fluctuation and load changes are depicted in Figure 6.13 


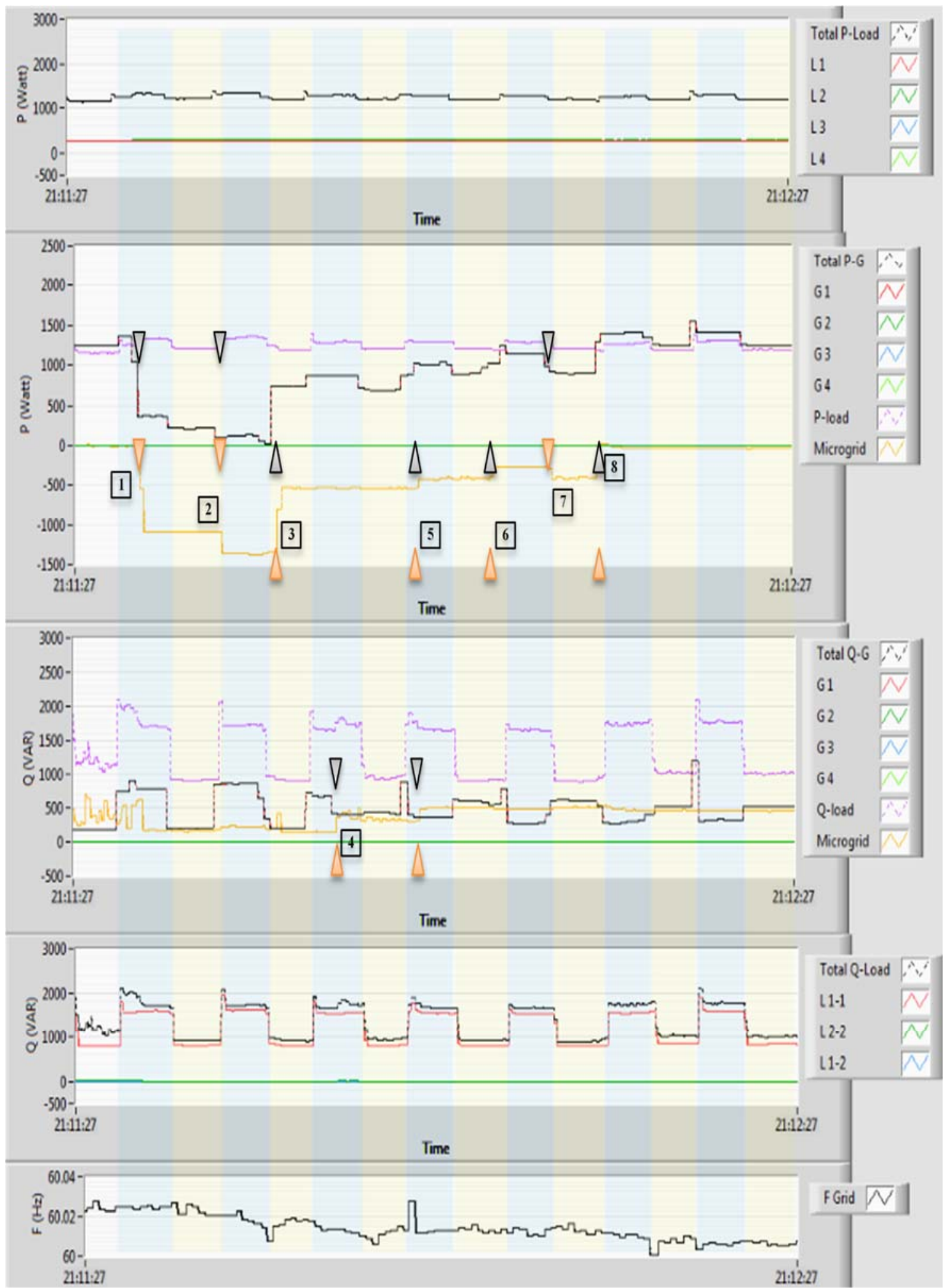

Figure 6.13 Active and reactive power in loads and generating units

Table 6.1 shows the steps followed in this experiment and response of the system. 
Table 6.1 Interconnection of AC/DC System in Power Sharing

\begin{tabular}{|l|l|l|l|l|}
\hline \multirow{2}{*}{$\begin{array}{l}\text { Event } \\
\text { Number }\end{array}$} & \multicolumn{2}{|c|}{ Event at PCC } & \multicolumn{2}{c|}{ System (slack bus) Response } \\
\cline { 2 - 5 } & $\begin{array}{l}\text { Injected } \\
\text { P (Watt) }\end{array}$ & $\begin{array}{l}\text { Injected } \\
\text { Q (VAR) }\end{array}$ & Generated P & Generated Q \\
\hline Before 1 & 0 & -350 & Balancing total P & Balancing total Q \\
\hline 1 & 1000 & -200 & Increase 1000 & Decrease 150 \\
\hline 2 & 1200 & -200 & Increase 200 & Constant \\
\hline 3 & 500 & -200 & Decrease 700 & Constant \\
\hline 4 & 500 & -400 & Constant & Increase 200 \\
\hline 5 & 500 & -500 & Constant & Increase 100 \\
\hline 6 & 350 & -500 & Decrease 150 & Constant \\
\hline 7 & 500 & -500 & Increase 150 & Constant \\
\hline 8 & 0 & -500 & Decrease 500 & Constant \\
\hline
\end{tabular}

With this experiment the interconnection of $\mathrm{AC} / \mathrm{DC}$ system for compensation of active and reactive power was demonstrated. During this experiment, by using integrated controllers for bidirectional converter, DC microgrids can support the AC grid generation through injecting the active/reactive power.

\subsubsection{Frequency Regulation of Islanded Microgrid}

In order to emulate frequency regulation and study the integrated DC side generation through bidirectional converter, the following structure was assumed and designed for experimental tests.

G2 and G4 are in the constant active power generation mode (PV mode) and the DC microgrid is connected through feeder " $0040 B$ ", applying the frequency control technique by injecting the active power to the $\mathrm{AC}$ microgrid. 


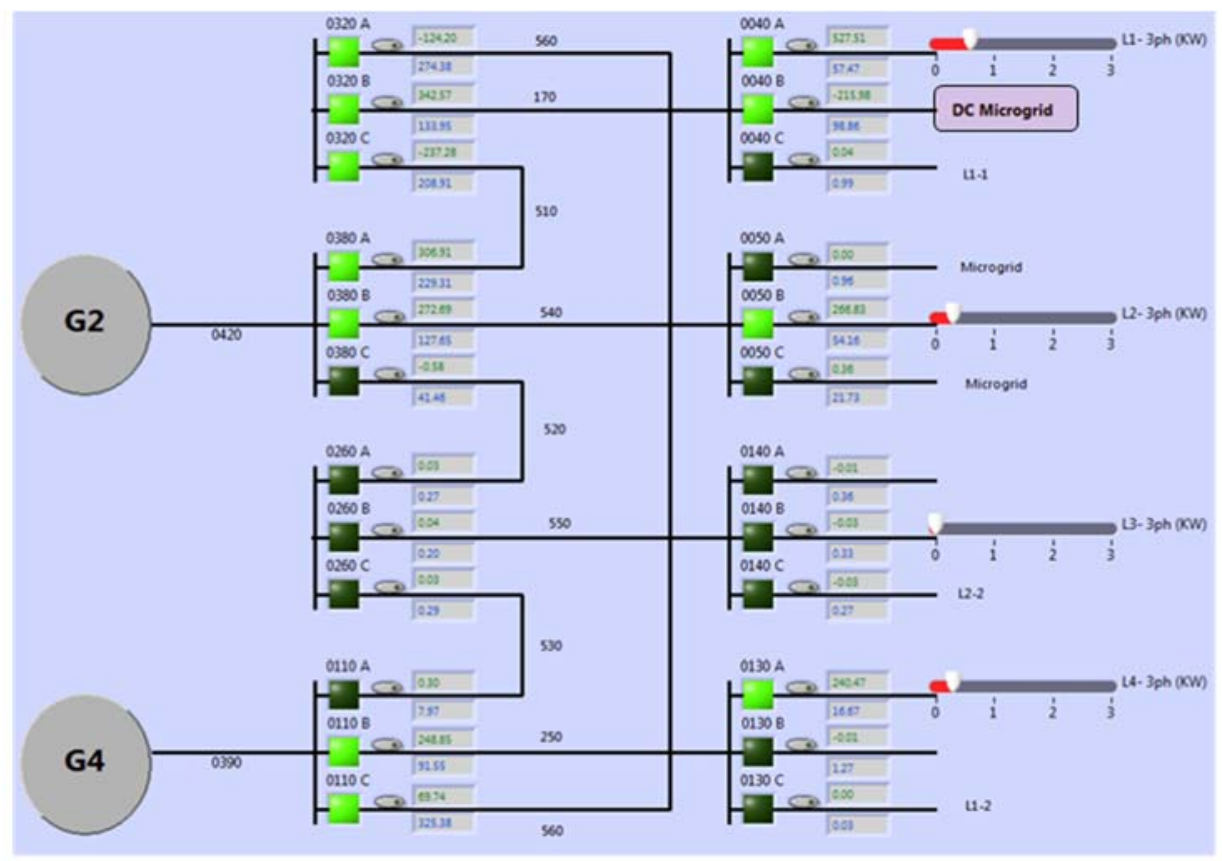

Figure 6.14 Test case power system for integrating electric car storage system into AC grid

Figure 6.15 shows the frequency regulation and steady state of $60 \mathrm{~Hz}$ for a $300 \mathrm{~W}$ step changes of the $L 1$ load. The generated power from the DC side is demonstrated in Figure 6.16 as negative load.

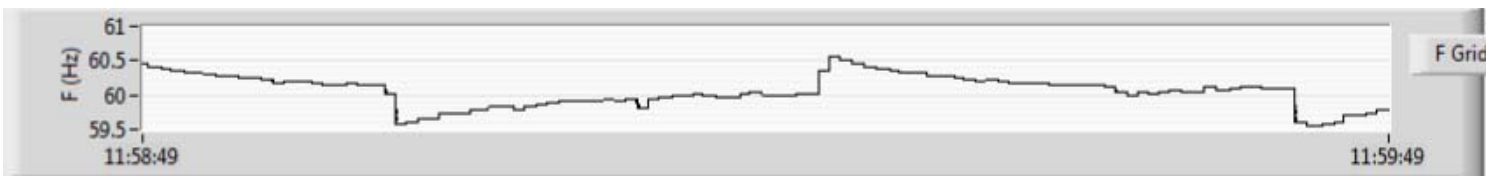

Figure 6.15 Frequency of microgrid regulated by DC side power management

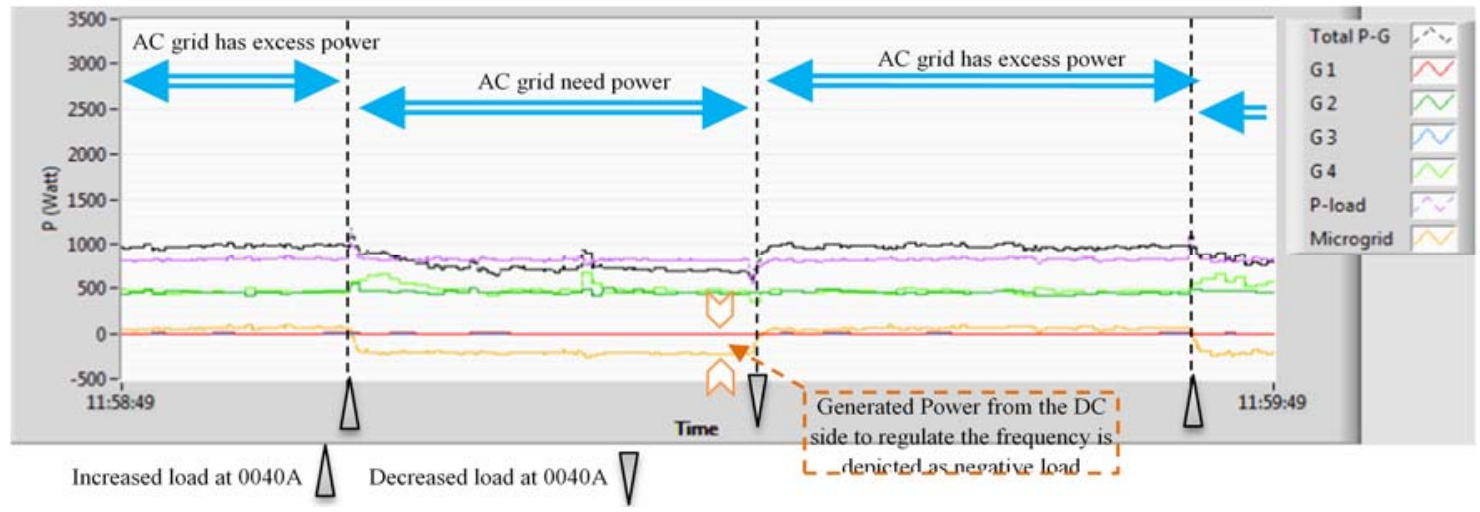

Figure 6.16 Variation of DC generation to regulate the frequency of the AC grid 


\section{THE REAL-TIME SMART GRID TESTBED}

\subsection{Introduction}

The need for evaluation and experimental testing of methodologies in operation, control, analysis, and improvement of real world issues of future power systems, as well as the need for real-time and actual integration of techniques urged the studies in this dissertation to be implemented on a close to actual and the most authentic mimic of power systems. One of the biggest distinctions between the current work and ongoing researches around the world is the viability of introducing methods in such practical way and through a provided unique platform of mimicked power system named as smart grid testbed.

Due to the requirements of the goals in this dissertation, a platform is needed to cover the fields of communication, real-time control and operation, energy management system, measurement and power system analysis.

A smart grid mimic is developed at the department of Electrical and Computer Engineering at Florida International University (FIU) to serve as an integrated hardwarebased AC/DC Hybrid power system, capable of producing power in AC and DC form and in particular for conducting the experimental investigations on variety of topics as well as the focus of this dissertation.

Figure 7.1 Overview of smart grid testbed 


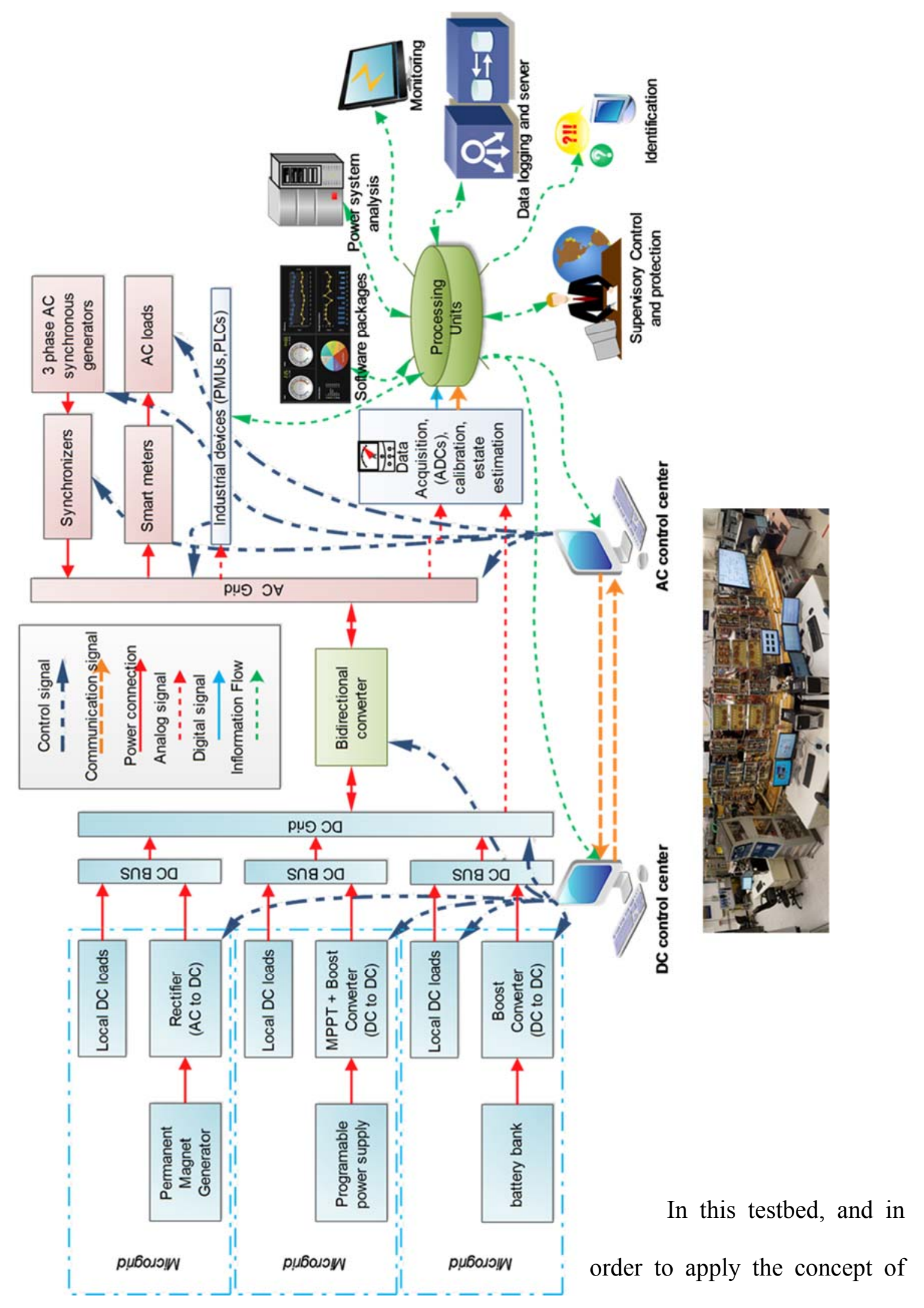

microgrids, the DC power is employed on several microgrids connected to the $\mathrm{AC}$ network. This hardware/software/communication-based system includes capabilities for 
the embedded systems, implementation of control strategies for generating stations, renewable source controllers and power transfer to programmable load hardware emulators. This system is an excellent platform at the laboratory scale to help test and verify new operation and control ideas. Appropriate software was developed to monitor all system parameters, as well as to operate and control the various interconnected components in varying connectivity architectures. The improvements of the testbed during this dissertation are depicted in Figure 7.2. In this chapter, the structure and components of the smart grid testbed will be explained.

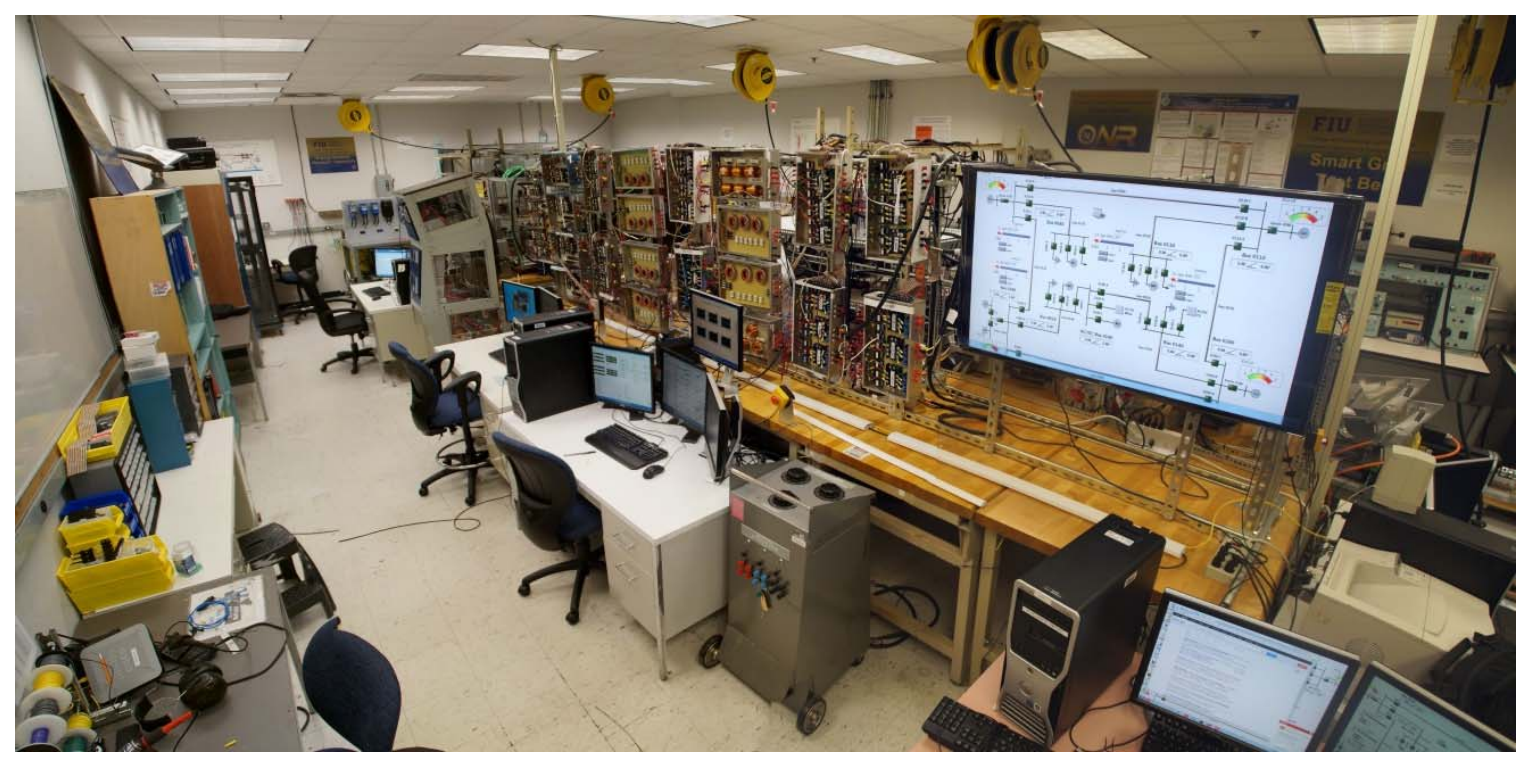

Figure 7.2 Smart grid testbed laboratory (as of 9/25/2013)

The smart grid testbed provides a reconfigurable laboratory environment and it is composed of 3 major substructures: generation, transmission/distribution and energy consumption.

The main advantages of this facility are real-time operation, flexibility of utility connection, integration and emulation of microgrids, and integration of several load models. 
The main components of smart grid testbed and their detailed composition will be explained in the following sections in order to support the practical integration of introduced methodologies in other chapters of this dissertation.

\subsection{Generation Station Model}

Generating stations are providing the electric power for the testbed. They are coupled to several prime movers by mechanical belts and electrically isolated from the source, which is the campus. The synchronous generators are rated $13.8-\mathrm{kVA}$ and 10.4 KVA, at 60-Hz, 230-V, 1800-RPM.

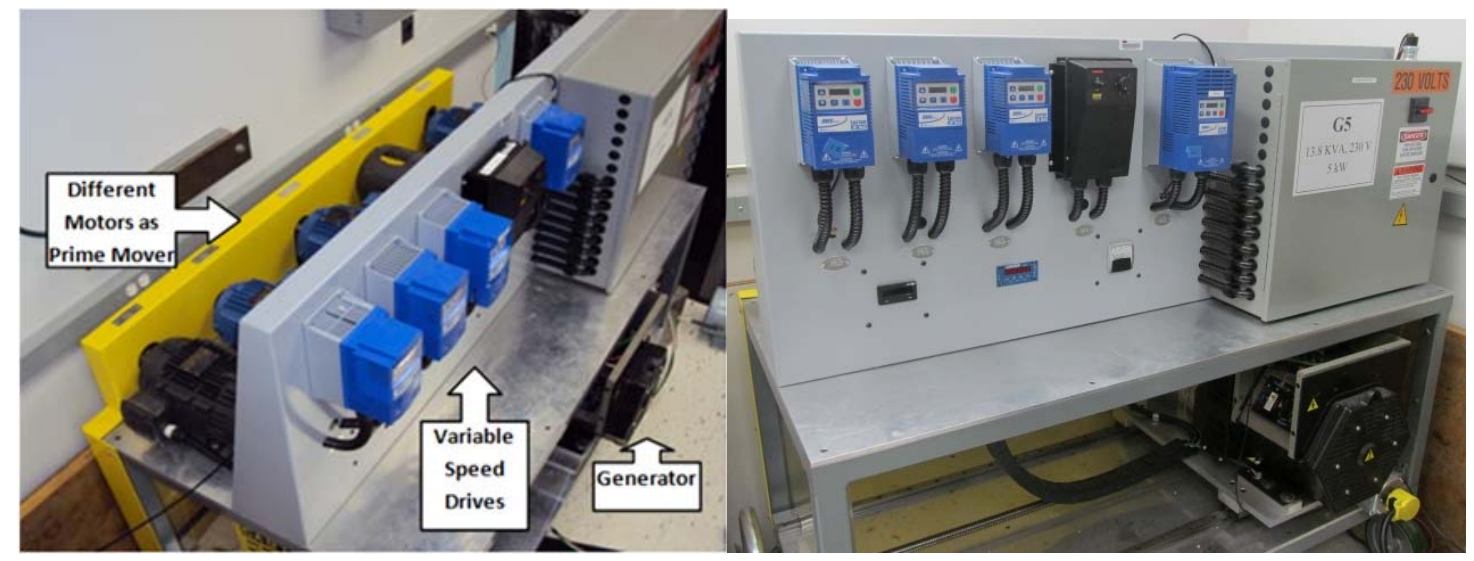

Figure 7.3 Generating stations

The prime mover of these generators can be one of the available asynchronous or direct-current motors, which are derived by different frequency drives. The prime movers consist of a selection of four of the rated 1, 2, 3, 4, 5.5 and 7.5 HP for four induction motors, and only a $2 \mathrm{HP} 180 \mathrm{~V}$ DC motor for each station. All of the synchronous generators are equipped with an Automatic Voltage Regulator (AVR) (model SX460) in order to maintain an output voltage magnitude. 


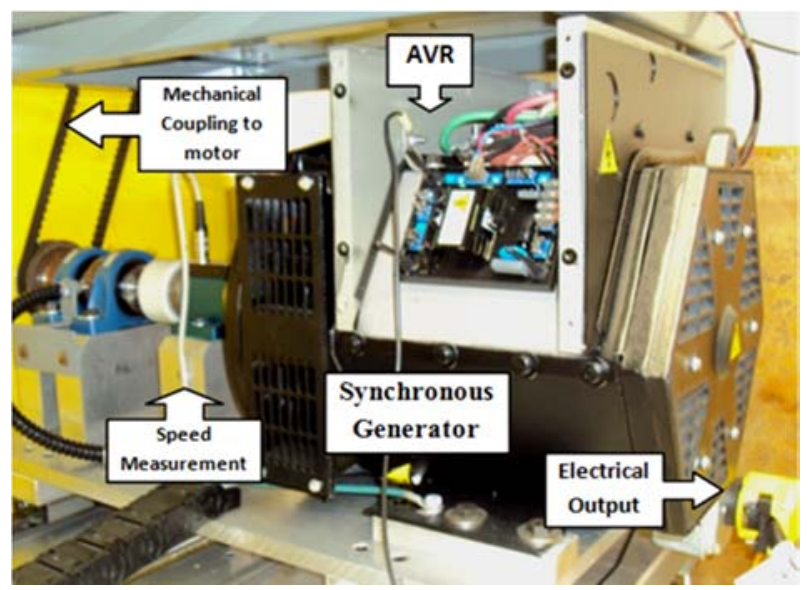

Figure 7.4 Synchronous generator and its accessories
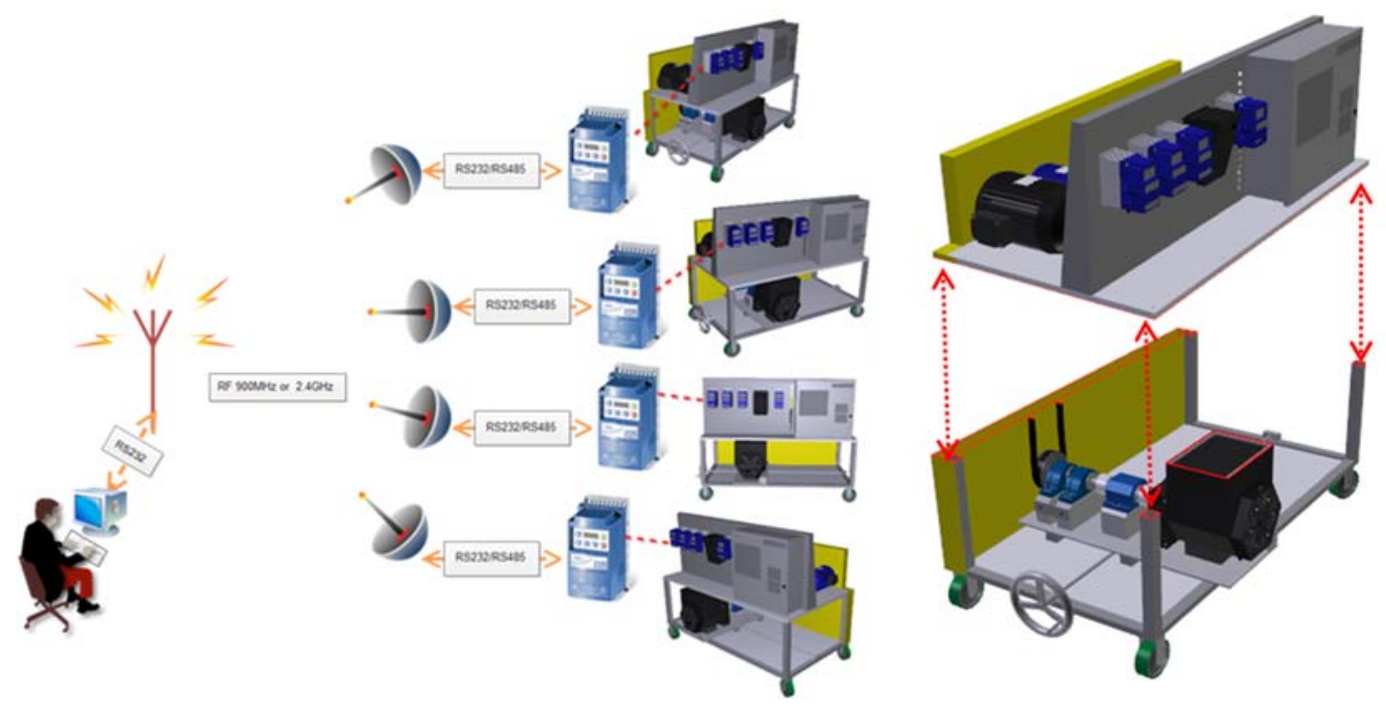

Figure 7.5 Generating stations

The SX460 AVR is a half-wave phase-controlled thyristor type AVR and forms part of the excitation system for a brush-less generator. In addition to regulating the generator voltage, the AVR circuitry includes under-speed and sensing loss protection features. Excitation power is derived directly from the generator terminals. 


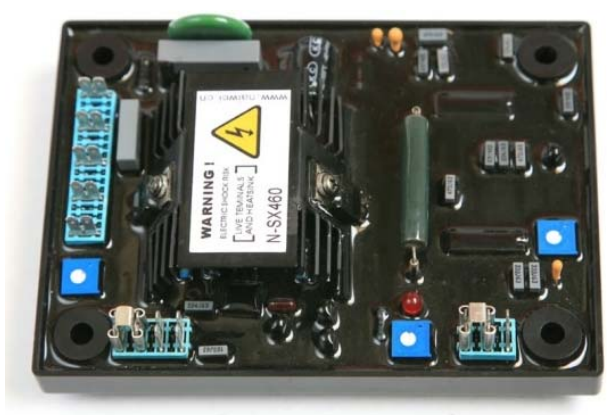

Figure 7.6 SX460 AVR module

Positive voltage builds up from a residual levels and it ensured by the use of efficient semiconductors in the power circuitry of the AVR. The AVR is linked with the main stator winding and the excitation field winding to provide closed loop control of the output voltage with load regulation of $+/-1.0 \%$. In addition to being powered from the main stator, the AVR also derives a sample voltage from the output winding for voltage control purposes. In response to this sample voltage, the AVR controls the power fed to the exciter field, and hence the main field, to maintain the machine output voltage within the specified limits, compensating for load, speed, temperature and power factor of the generator [104]. 


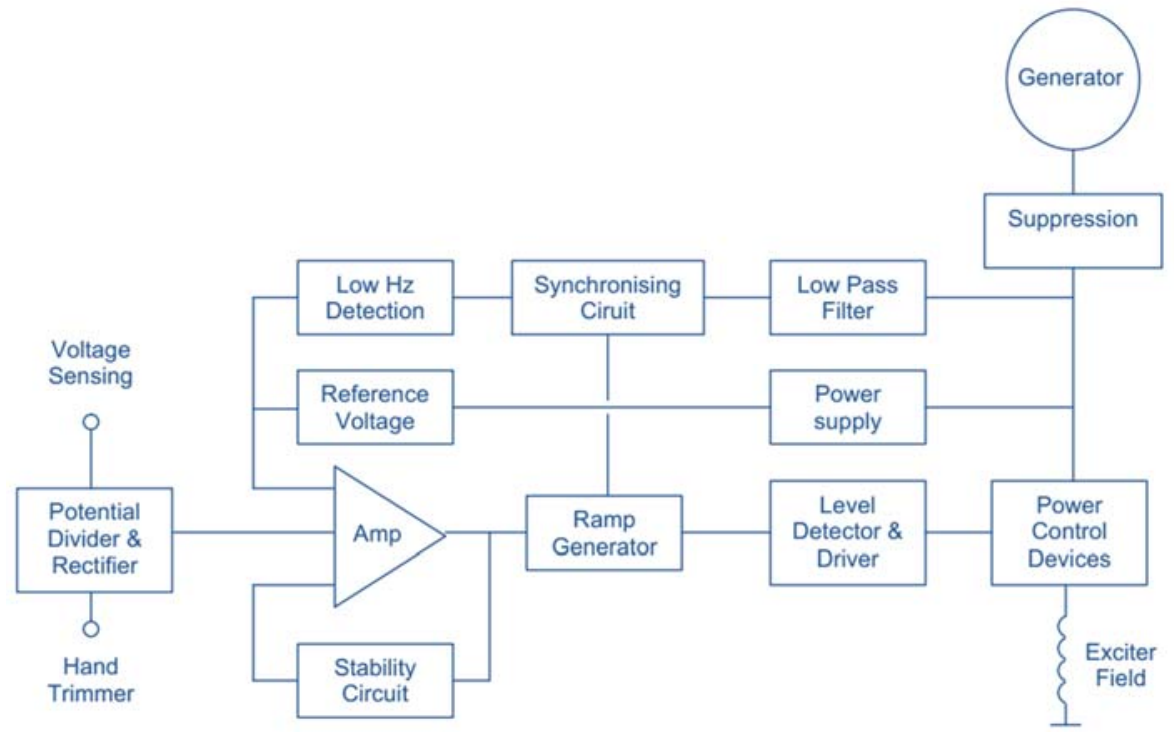

Figure 7.7 Internal schematic of SX460

Figure 7.7 demonstrates the internal schematic of SX460 AVR and its specification is available in Table 7.1 [104].

Table 7.1 SX460 Specifications

\begin{tabular}{|l|l|}
\hline Parameter & Value \\
\hline Input voltage: & $\begin{array}{l}\text { 95-132Vac or 190-264Vac } \\
\text { Single phase, } \\
\text { Frequency: 50-60 Hz }\end{array}$ \\
\hline Max Output Voltage: & 90Vdc @ 207Vac input \\
\hline Max Output Voltage: & 4Adc (intermittent 6Adc for 10 Seconds) \\
\hline Regulation: & $+/-1 \%$ \\
\hline System response: & $\begin{array}{l}\text { AVR Response: } 20 \mathrm{~ms} \\
\text { Field current to } 90 \%: 80 \mathrm{~ms} \\
\text { Machine volts to 97\%: 300ms }\end{array}$ \\
\hline Unit Power Dissipation: & 10 Watts \\
\hline Build up Voltage & 4V @ AVR terminal \\
\hline
\end{tabular}

The frequency inverter drive used for prime movers of generating stations is the LENZE AC Tech SMVector series and can be used with 3-phase AC induction motors rated from $1 / 3 \mathrm{HP}$ up to $25-\mathrm{HP}$ on voltages from $120 \mathrm{-V}$ single-phase to $600-\mathrm{V}$ three- 
phases. They can be communicated through digital and analog I/O channels of the drive to be applicable for many application-specific tasks, such as multiple preset speeds, electronic braking and motor jogging.

For implementing a smart grid with control, the original program named TECHLINK was used in order to control the frequency and change the output active power of generators. The following figure is showing the application software to control the Lenze Drives.
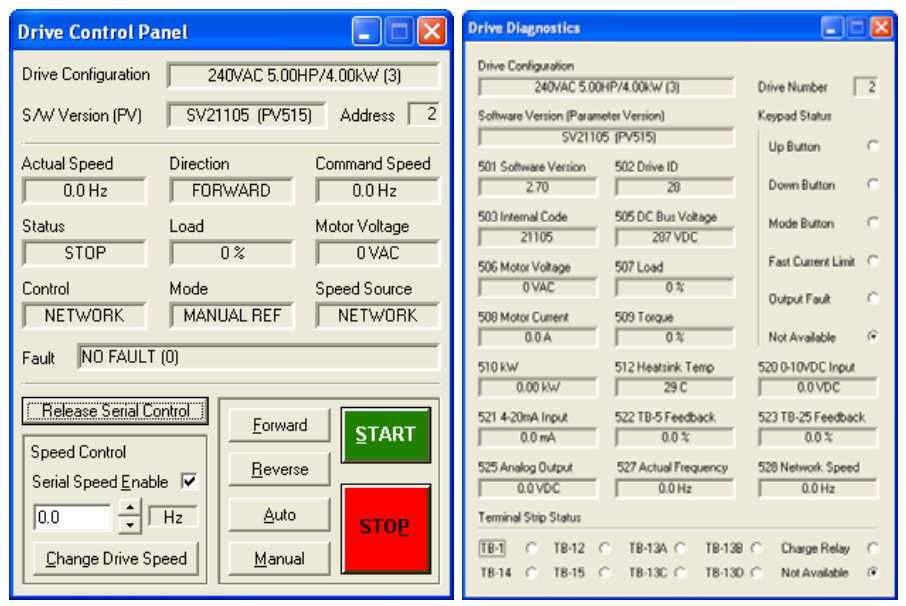

Figure 7.8 TECHLINK interface software

TECHLINK software has limitations, which does not allow us to perform control strategies, so there is a need to create a practical software for testbed application.

LENZE drives have several control modes (such as Constant V/Hz, Variable $\mathrm{V} / \mathrm{Hz}$, enhanced $\mathrm{V} / \mathrm{Hz}$, Vector speed, and Vector torque) and based on our needs, we have selected the following:

7.2.1. Vector Speed 
This mode is good for single-motor applications requiring higher starting torque and also for speed regulation, such as working as the slack bus, which requires constant speed and consequently constant frequency.

\subsubsection{Vector Torque}

This mode is good for single-motor applications, requiring torque control independent of speed, and for our application working as constant output power of mechanically coupled synchronous generators, which are grid connected and have constant speed regulated by the grid.

Slack bus is responsible for controlling the frequency of the grid and PV and PQ buses participate in power generation within the power grid. Since the slack bus has to have constant frequency, it is required to have a frequency control mode for the slack bus. Both PV and PQ buses have constant generated active power and since the grid usually has constant frequency (provided by the slack bus), they can be used as a constant power generator, by working at the torque control mode.

Figure 7.9 shows the schematic of the control loop for generating stations in the testbed. 


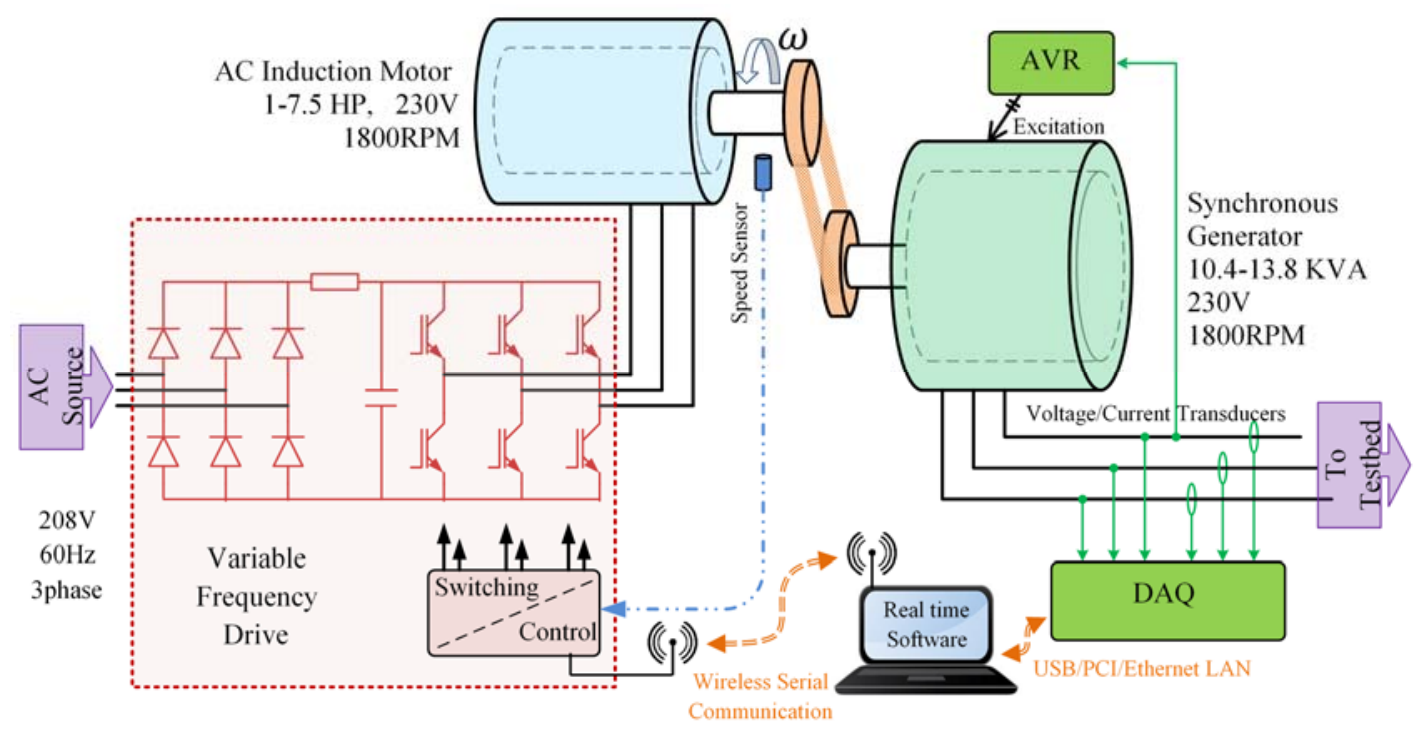

Figure 7.9 Schematic of a generator station control loop

Synchronous generators, which have been integrated, are known as the SLG164B type and their technical parameters are available in Table 7.2.

For the purpose of communication, control and operation of generation stations, a unique software was developed in the LabVIEW environment. This real-time system will be explained in the next chapter in detail.

Table 7.2 Parameters of Synchronous Generators

Parameter name

Generator 1,3 Generator 2,4

\begin{tabular}{llcc}
\hline \hline Aparent Power & $\mathrm{S}_{\text {Nom. }}$ & $13.8 \mathrm{KVA}$ & $10.4 \mathrm{KVA}$ \\
Nominal Voltage & $\mathrm{V}_{\text {Vom. }}$ & $230 \mathrm{~V}$ & $230 \mathrm{~V}$ \\
Nom. Power Factor & $\mathrm{PF}_{\text {Nom. }}$ & 0.8 & 0.8 \\
Rotor Type & & Salient Pole & Salient Pole \\
Acceleration Time Constant & $\mathrm{T}$ & $0.005625 \mathrm{~s}$ & $0.004 \mathrm{~s}$ \\
Stator Leakge Resistance & $\mathrm{X}_{\mathrm{RL}}$ & $0 \mathrm{pu}$ & $0 \mathrm{pu}$ \\
Stator Leakge Reactance & $\mathrm{X}_{\mathrm{L}}$ & 0.09 & 0.09 \\
Direct Axis Synchronous Reactance & $\mathrm{Xd}$ & $2.21 \mathrm{pu}$ & $2.248 \mathrm{pu}$ \\
Quadrature Axis Synchronous Reactance & $\mathrm{Xq}$ & $1.1 \mathrm{pu}$ & $1.117 \mathrm{pu}$
\end{tabular}




\begin{tabular}{llcc} 
Direct Axis Transient Time Constant & Td' & $0.014 \mathrm{~s}$ & $0.012 \mathrm{~s}$ \\
Direct Axis Transient Reactance & Xd' & $0.23 \mathrm{pu}$ & $0.23 \mathrm{pu}$ \\
& & & \\
Direct Axis Subtransient Time Constant & Td" & $0.0035 \mathrm{~s}$ & $0.0028 \mathrm{~s}$ \\
Quadrature Axis Subtransient time Constant & Tq" & $0.05 \mathrm{~s}$ & $0.038 \mathrm{~s}$ \\
Direct Axis Subtransient Reactance & $\mathrm{Xd} "$ & $0.14 \mathrm{pu}$ & $0.144 \mathrm{pu}$ \\
Quadrature Axis Subtransient Reactance & Xq" & $0.25 \mathrm{pu}$ & $0.258 \mathrm{pu}$ \\
\hline
\end{tabular}

\subsection{Transmission Line Model}

All transmission lines are designed for three phases, 5-wire connection (3 phases, Neutral and Ground) and based on the $\pi$-model of actual overhead or cable lines, as in Figure 7.10. Each line has an enclosure, which is grounded for Electro-Magnetic Interference (EMI) and noise issues.
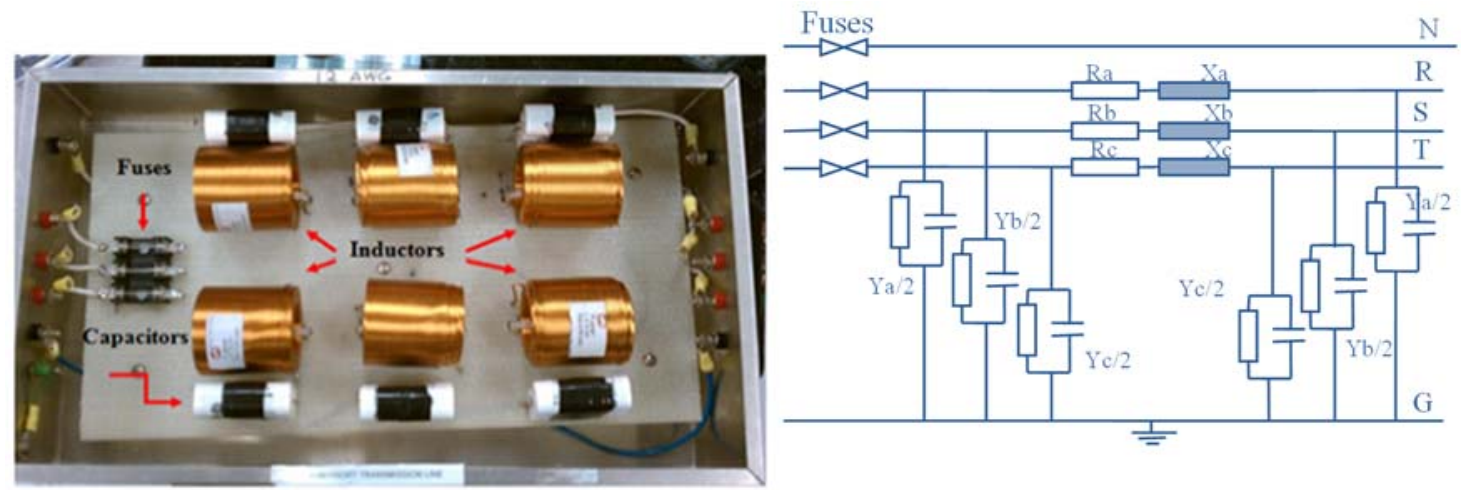

Figure 7.10 Developed transmission line model and schematic diagram of the 3 phase $\pi$-model

For protection of the circuit, in the case of fault or long-term overloads, they are equipped with fuses to prevent the components from being damaged. The components, which have been included in each transmission line, are solenoid or toroidal inductors with internal resistivity for series branch, along with AC capacitors for parallel branches. The rated design for each line is $132.8 \mathrm{~V}$ and 10 or $15 \mathrm{~A}$ per-phase, and consequently, the maximum power transferring capacity will be 5976VA or 3984VA, correspondingly. 
Different combinations of transmission lines can provide a power grid with a variety of architecture, lengths and power ratings.

\subsection{Bus Model}

The Bus Model is designed for the purpose of working as a breaker (switching purposes) and Remote Terminal Units (RTU) (Data Acquisition purposes). For these purposes, the buses are equipped with solid state switches and Current Transformers (CTs) and Voltage Transformers (VTs) (Figure 7.11). Solid states switches are rated 25 or $50 \mathrm{~A}, 530 \mathrm{~V}$. They are fast response, simultaneous 3phase-switching, and they are working with low voltage (3-32VDC) and low power consumption of less than $40 \mathrm{~mA}$. These characteristics make them a great option in contradiction of electro-mechanical switches. The CTs are rated 3A-to-1V and the PTs are rated 20V-to-1V. Since the system is in 3 phases, each switch has 3 current, and 3 voltage transducers. The secondary side of CTs and PTs are connected to Data Acquisition (DAQ) terminals.

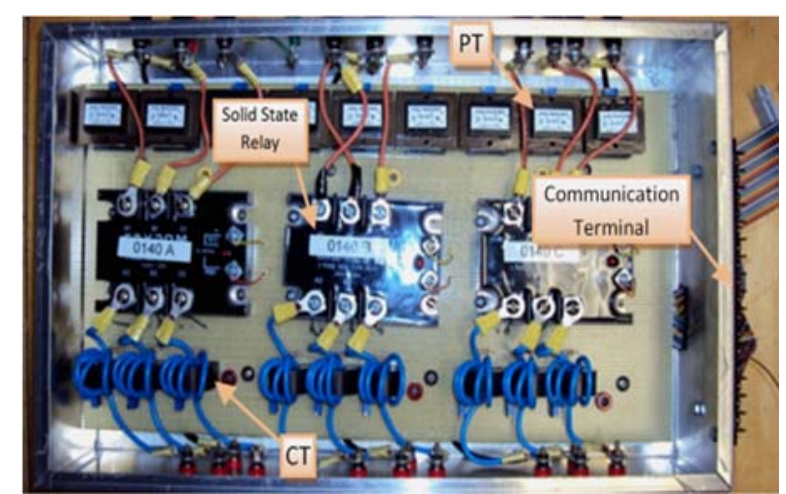

(a)

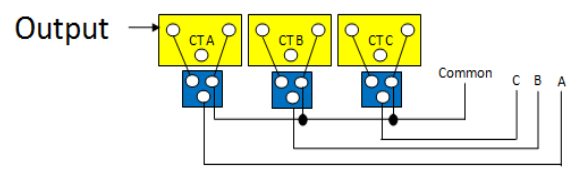

(c)

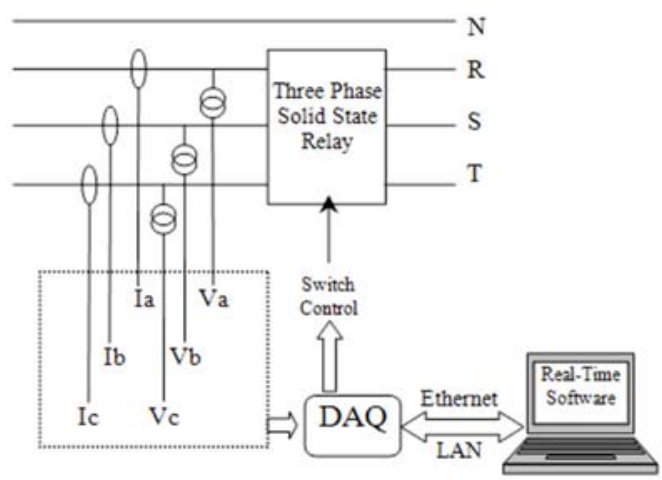

(b)

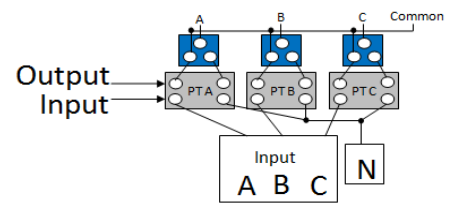

(d) 
Figure 7.11 Bus model (a) Developed bus model with 3 feeders (b) Overview of each 3 phases input (feeder), (c) Electrical connection of CTs, (d) Electrical connection of PTs

\subsection{Load Model}

A variety of load types have been integrated in the testbed. In this section, we will introduce these loads, their design, and the implementations.

\subsubsection{Dynamic Motor Loads}

The induction motors are used for dynamic model of loads, and they are coupled to a Prony-brake in order to apply different mechanical loads on their shaft. The motors used are $208 \mathrm{~V}, 1.4 \mathrm{~A}, 1 / 3 \mathrm{HP}$ induction machines. A remote controllable load has also been developed in order to control the load value by its software in real-time control. According to the system requirement, the load level can be set by changing the reference signal. This kind of the load controlling makes the smart grid concept more operational and achievable. The motor load emulator system is shown in Figure 7.12. As it is shown, this system consists of one $250 \mathrm{~W}$ induction machine, operating as an induction motor load, one $250 \mathrm{~W}$ DC machine, operating as a separately excited DC generator, a resistive load connected to the DC generator, an IGBT for controlling the field of the DC generator, and finally a free-wheeling diode for circulating the field current, when the IGBT is open. An anti-windup PI controller provides the proper duty cycle for the IGBT operation. This duty cycle will be applied to the IGBT via the Gate block, which converts the duty cycle to the appropriate gating signals. 


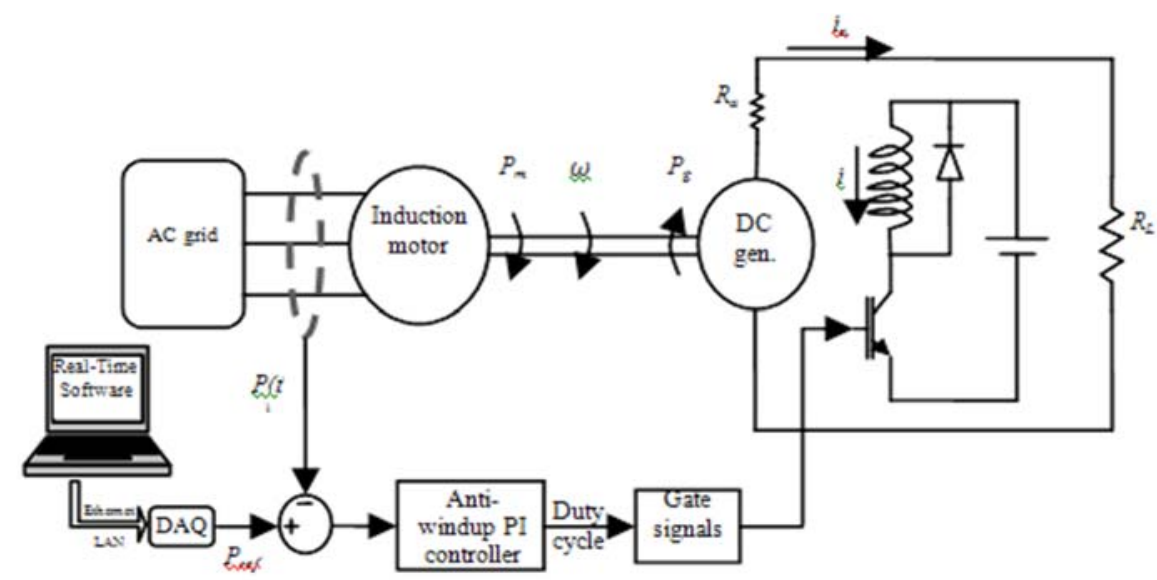

Figure 7.12 Schematic diagram of motor load emulator

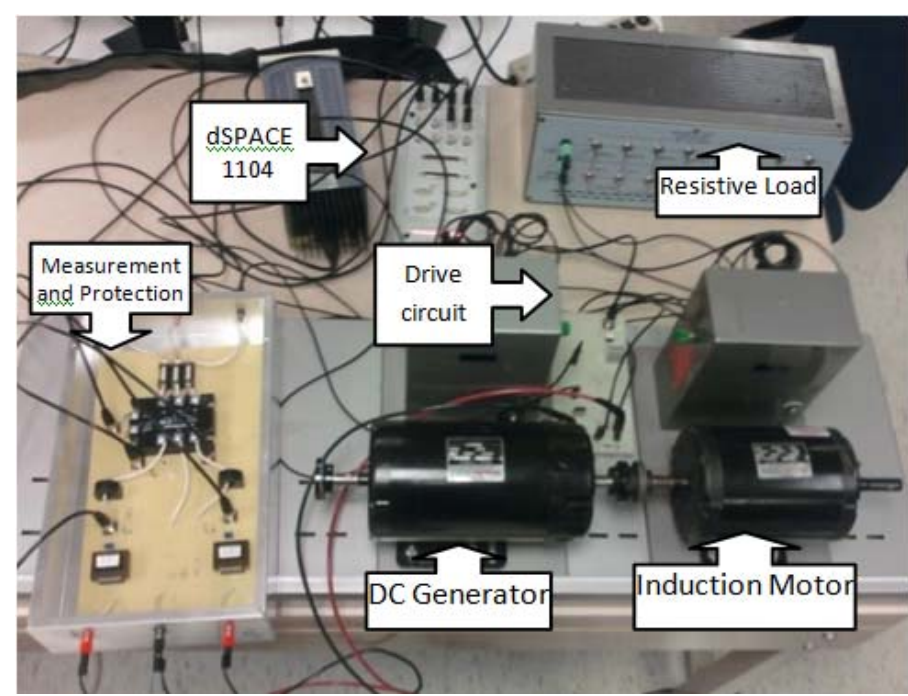

Figure 7.13 Developed motor load emulator model

\subsection{1. $\quad$ Resistive Load}

In order to emulate the resistive load on the power system, this load was designed with several characteristics, which make it more beneficial for our studies. Table 7.3 shows the specifications of the resistive loads.

Table 7.3 Specifications of Developed Resistive Loads

\begin{tabular}{lc}
\hline \multicolumn{1}{c}{ Parameters } & Values \\
\hline \hline Total Number of Loads & 4 \\
Total Number of Load boxes & 2
\end{tabular}


Type of Load

Type of Breaker Switch

Number of Switches in each load

Total Number of Control steps in each load

Load Step

Total Active power of each load

Total Active power of all loads

Operating Voltage

Total material Cost for all loads
3 phase, Static, Constant Resistor

3 phase solid state switch

6

10

$300 \mathrm{~W}$

$3 \mathrm{KW}$

$12 \mathrm{KW}$

208 VAC (3 phase)

Less than $3000 \$$

Figure 7.14 shows the implemented load box. There are two load boxes; each contains 2 independent resistive loads, which can be controlled by the software interface, and developed in the testbed laboratory through the NI DAQ Digital Output modules.
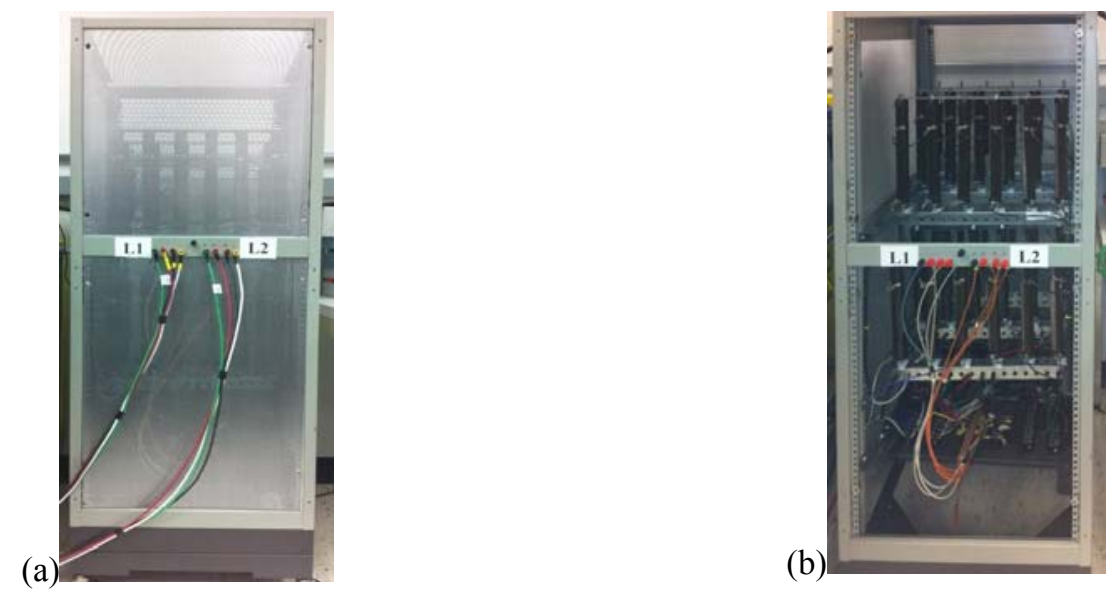

Figure 7.14 Load box , (a) Each load box with 2 independent loads (b) Internal components (Resistors and switches) 


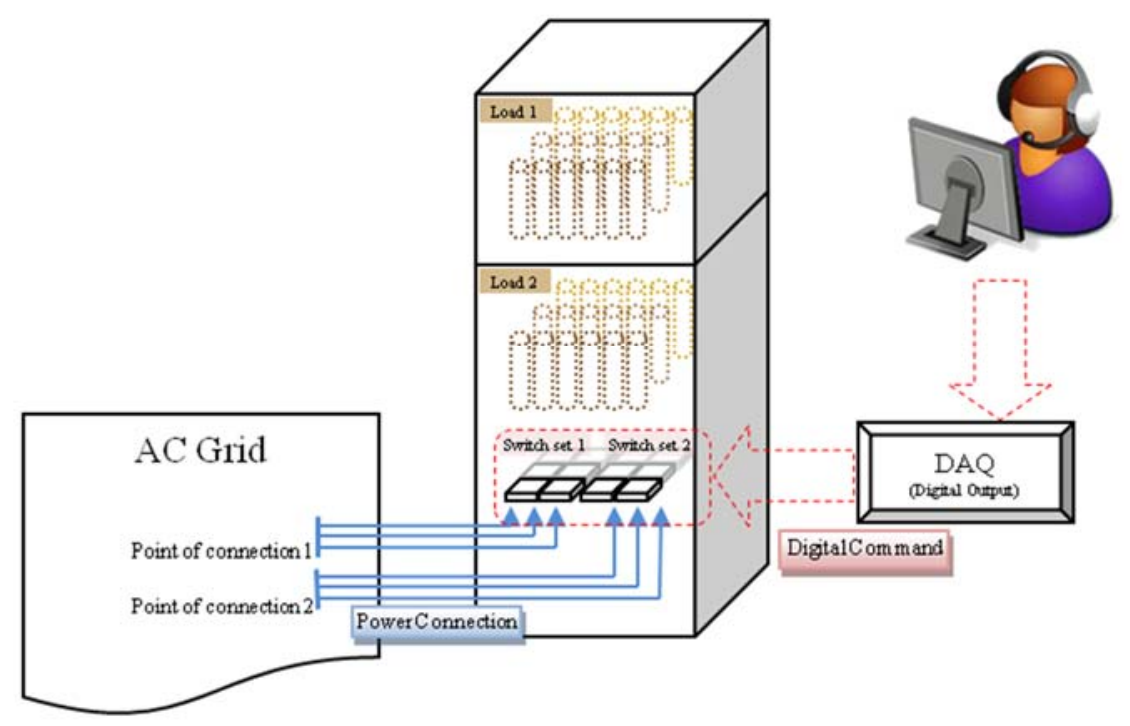

Figure 7.15 Resistive load communication and connectivity scheme

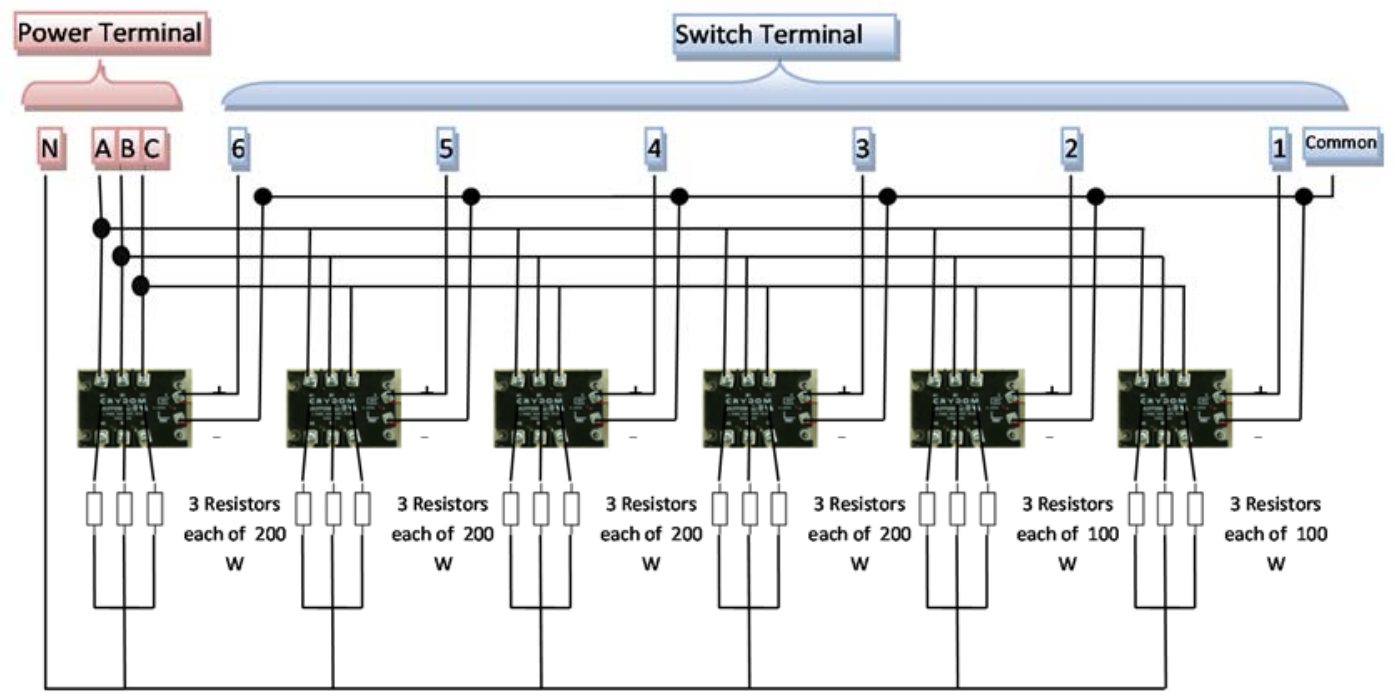

Figure 7.16 Internal connection of each resistive load

\subsubsection{Inductive Load}

The inductive loads have been designed to provide reactive power consumption in the testbed. The design is based on constant inductance, so they will be similar to 
constant impedance loads in the case that the system frequency is constant. Otherwise, the frequency drift will change the inductance and this needs to be considered in the analysis. Figure 7.17 shows the implemented inductive load box. The electrical connection of each inductive load is depicted in Figure 7.18. Based on this design, the inductive load has the specifications, which are available in Table 7.4.
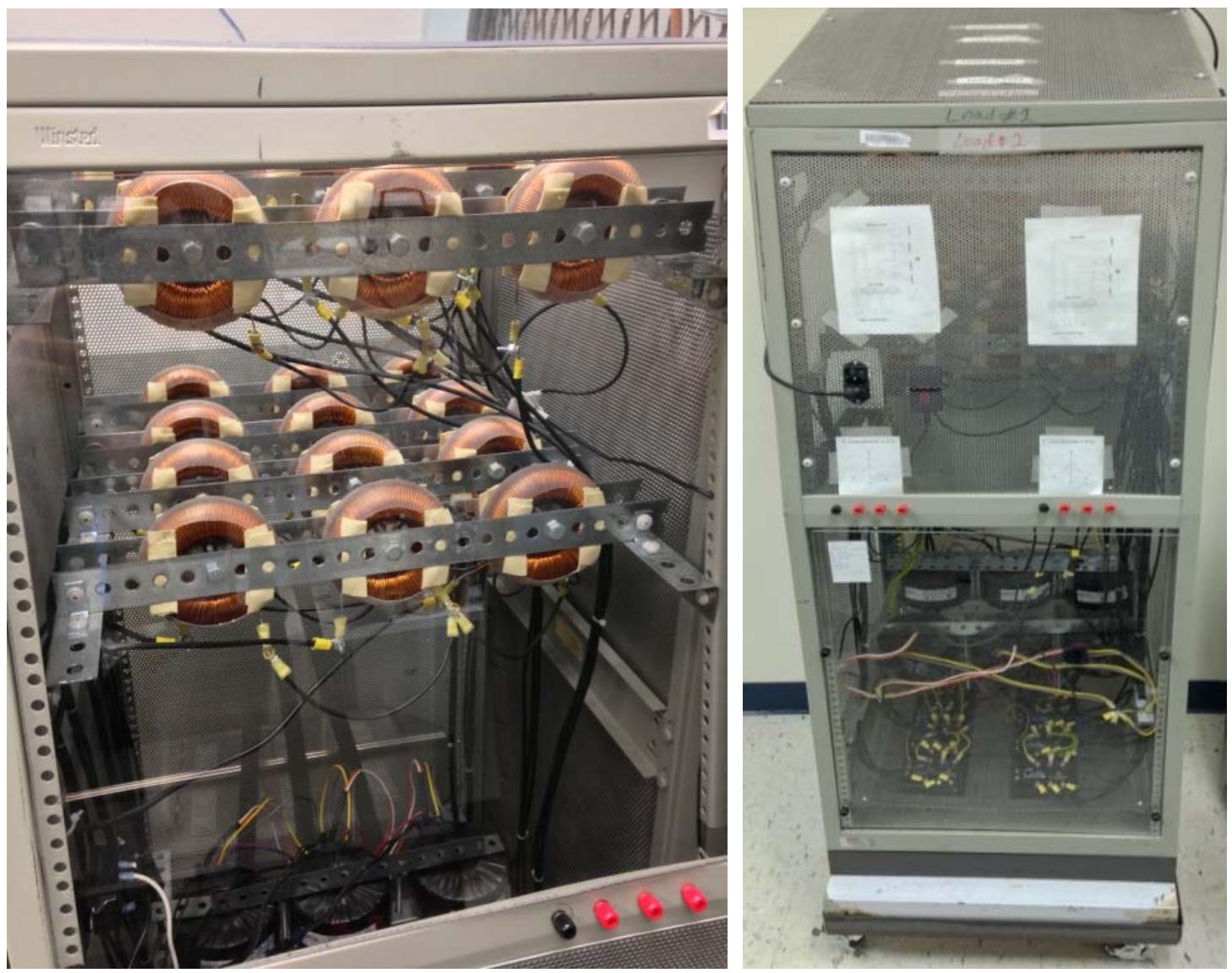

Figure 7.17 Inductive load box contains 2 individual loads

This load is designed to be available not only for the LabVIEW environment. Therefor it has a control circuit, which receives the command and applies the proper load, respectively. 


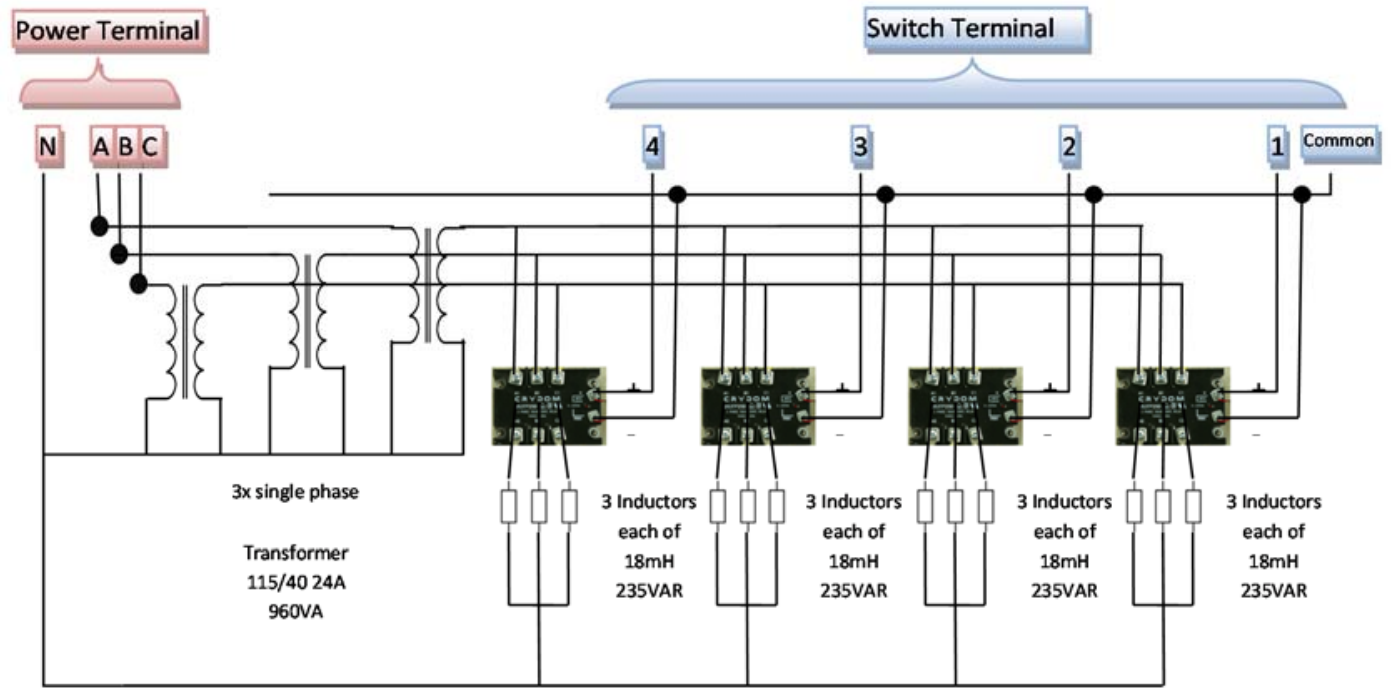

Figure 7.18 Internal connection of each inductive load

Table 7.4 Specification of Inductive Loads

\begin{tabular}{lc}
\hline \multicolumn{1}{c}{ Parameters } & Values \\
\hline \hline Total Number of Loads & 4 \\
Total Number of Load boxes & 2 \\
Type of Load & 3 phase, Static Inductive Load \\
Type of Switch & 3 phase solid state switch \\
Number of Switches in each load & 4 \\
Total Number of Control steps in each & 4 \\
load & 705 VAR \\
Load step & 2820 VAR \\
Total Active power of each load & 11280 VAR \\
Total Active power of all loads & 208 VAC (3 phase) \\
Operating Voltage &
\end{tabular}

Two circuits have been designed for this controller. The first circuit has the dsPIC micro controller and communicates through the RS232 (UART) channel. 


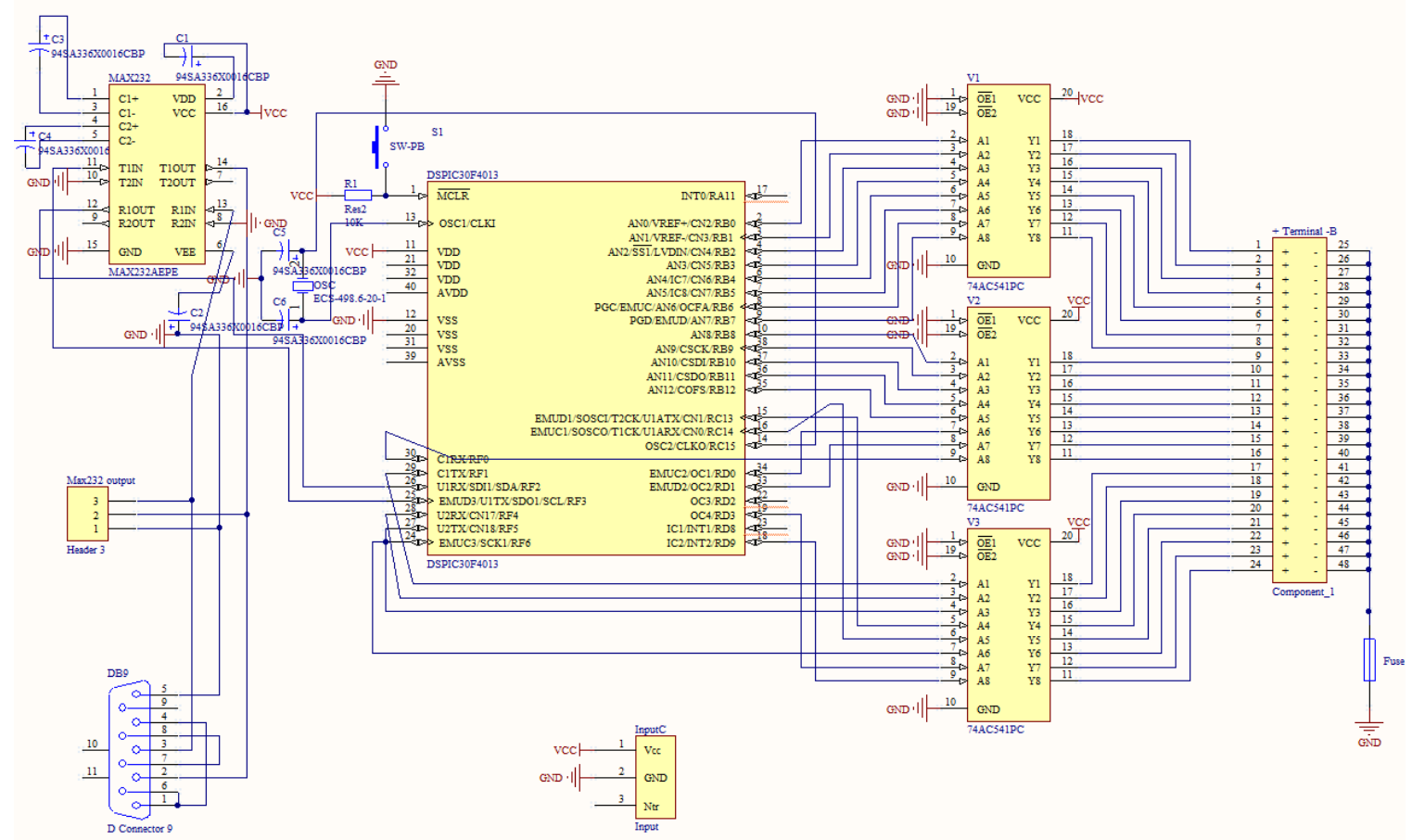

Figure 7.19 Schematic diagram of the first control circuit for inductive loads
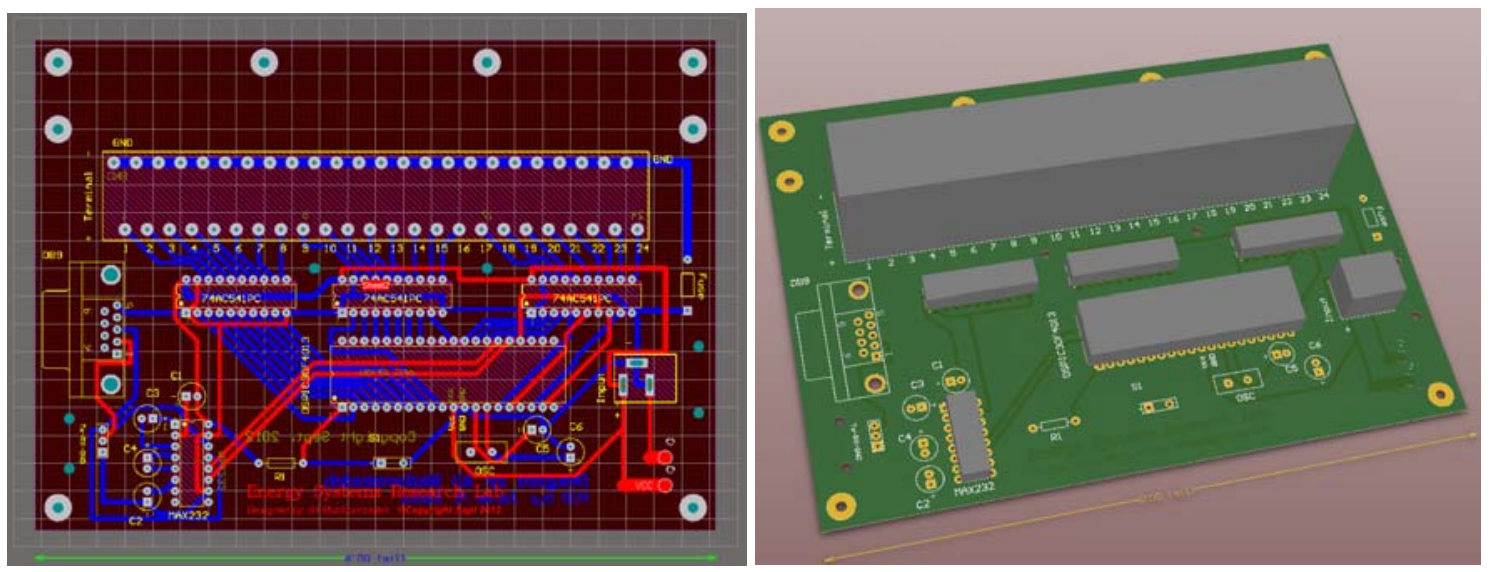

Figure 7.20 PCB design and pre-manufacturing progress

In order to improve the performance and the flexibility/enhancement of programming the microcontroller, a new platform of embedded system was integrated for several applications in the testbed. This hardware platform consists of a development board, which is named STM32F4 Discovery, and is equipped with a STM32F407 ARM Processor and a programming kit named Waijung Blockset, which will be added to 
Matlab Simulink. The main benefit of this platform is integration of the programming by the Matlab Simulink, which provides us quick and flexible programming features along with minimum possible script-programming.

The second circuit integrating STM32 is depicted in Figure 7.21. For the facilitation of integrity in the testbed, this load can be controlled through XBEE wireless communication.

The connection map of the inductive load, along with the control circuit and communication, is depicted in Figure 7.22. Figure 7.23 shows the Simulink program for inductive load module, and finally, the code is downloaded to STM32.

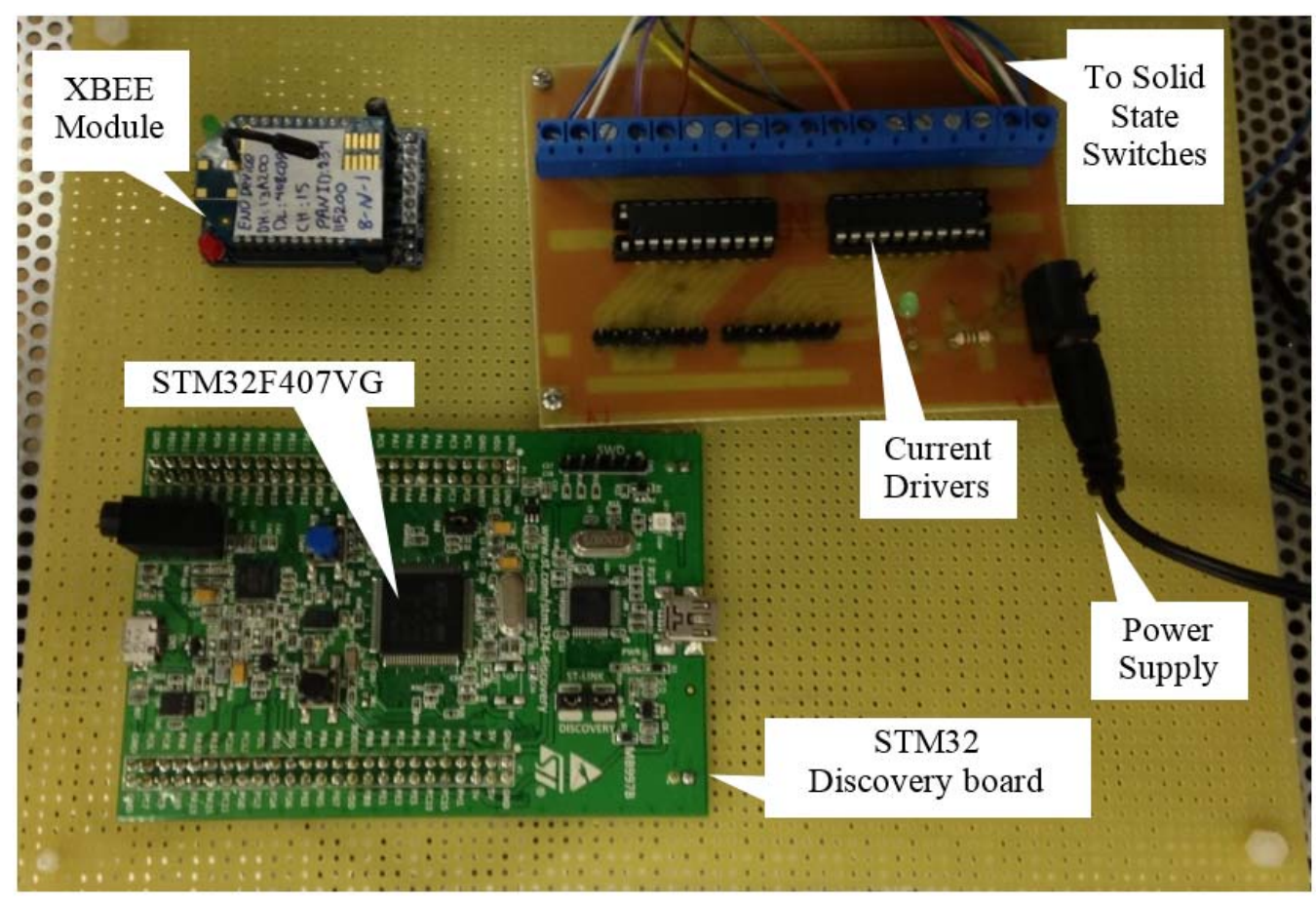

Figure 7.21 Inductive load controller equipped with XBEE and STM32F4 Processor 
Top Loads

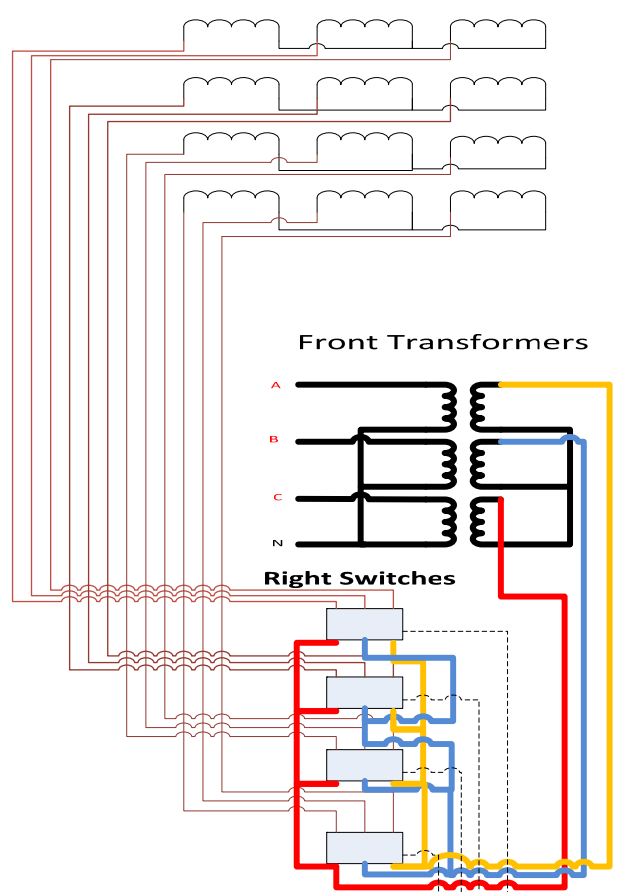

Bottom Loads

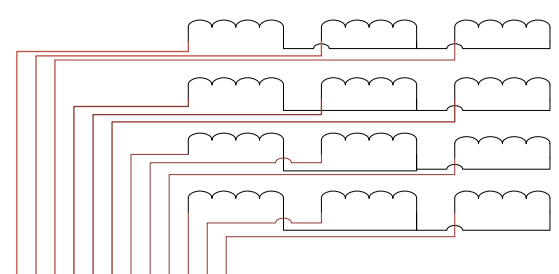

Back Transformers
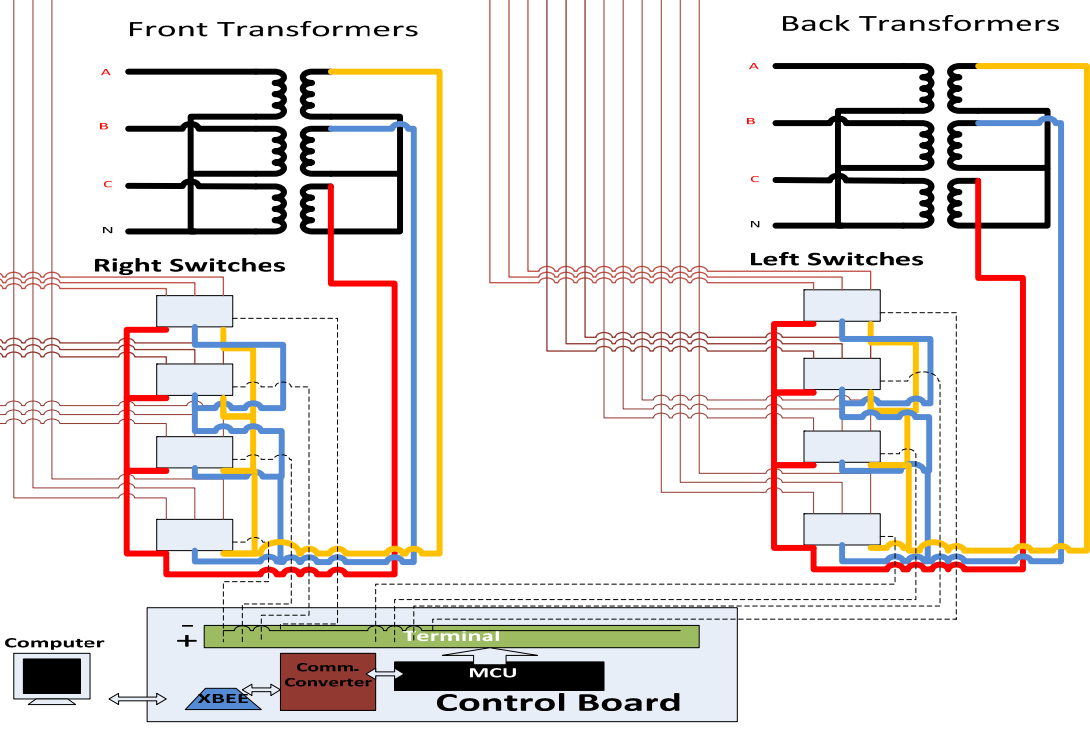

Figure 7.22 Inductive load box and control scheme

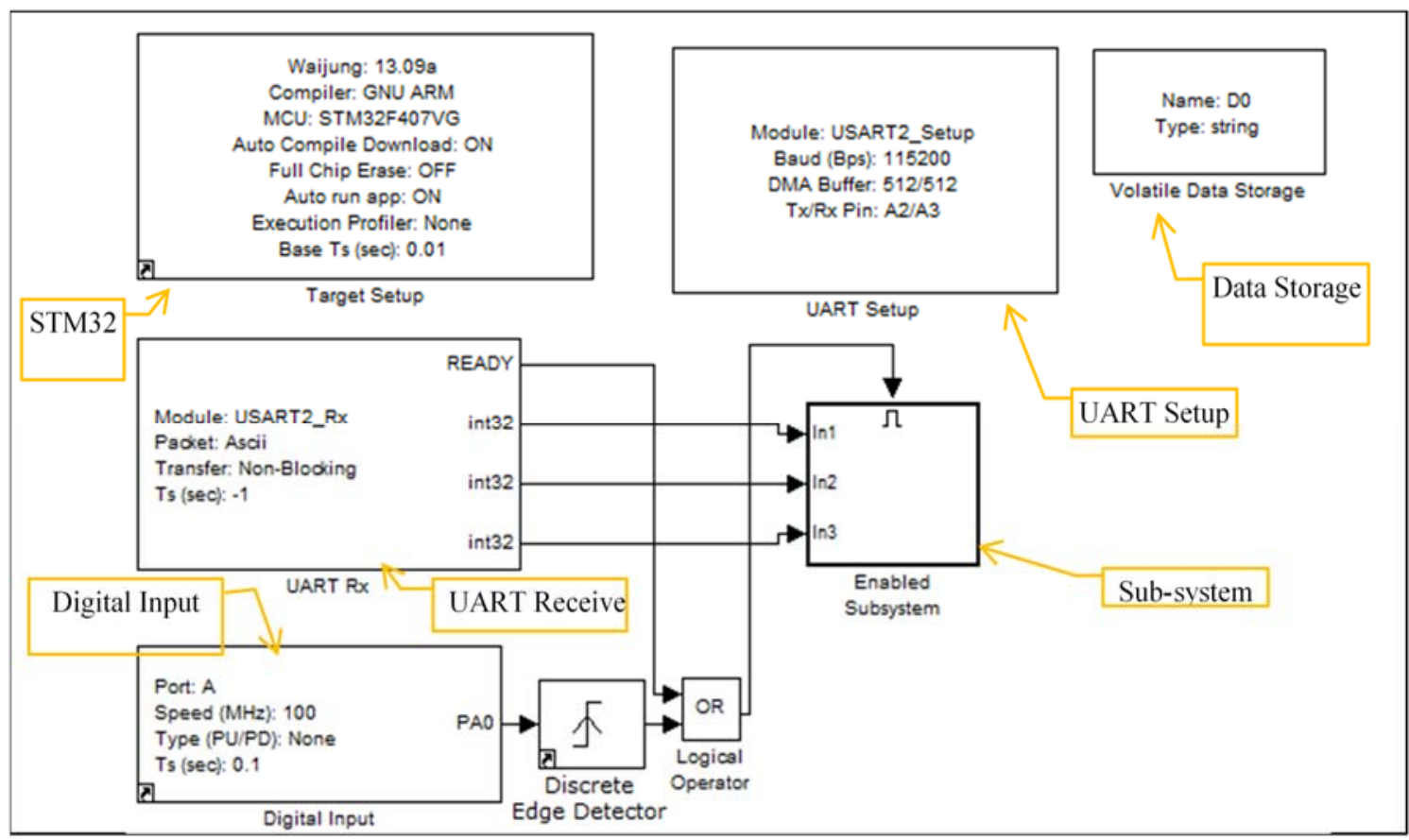

Figure 7.23 Simulink model for inductive load controller and communication circuit 


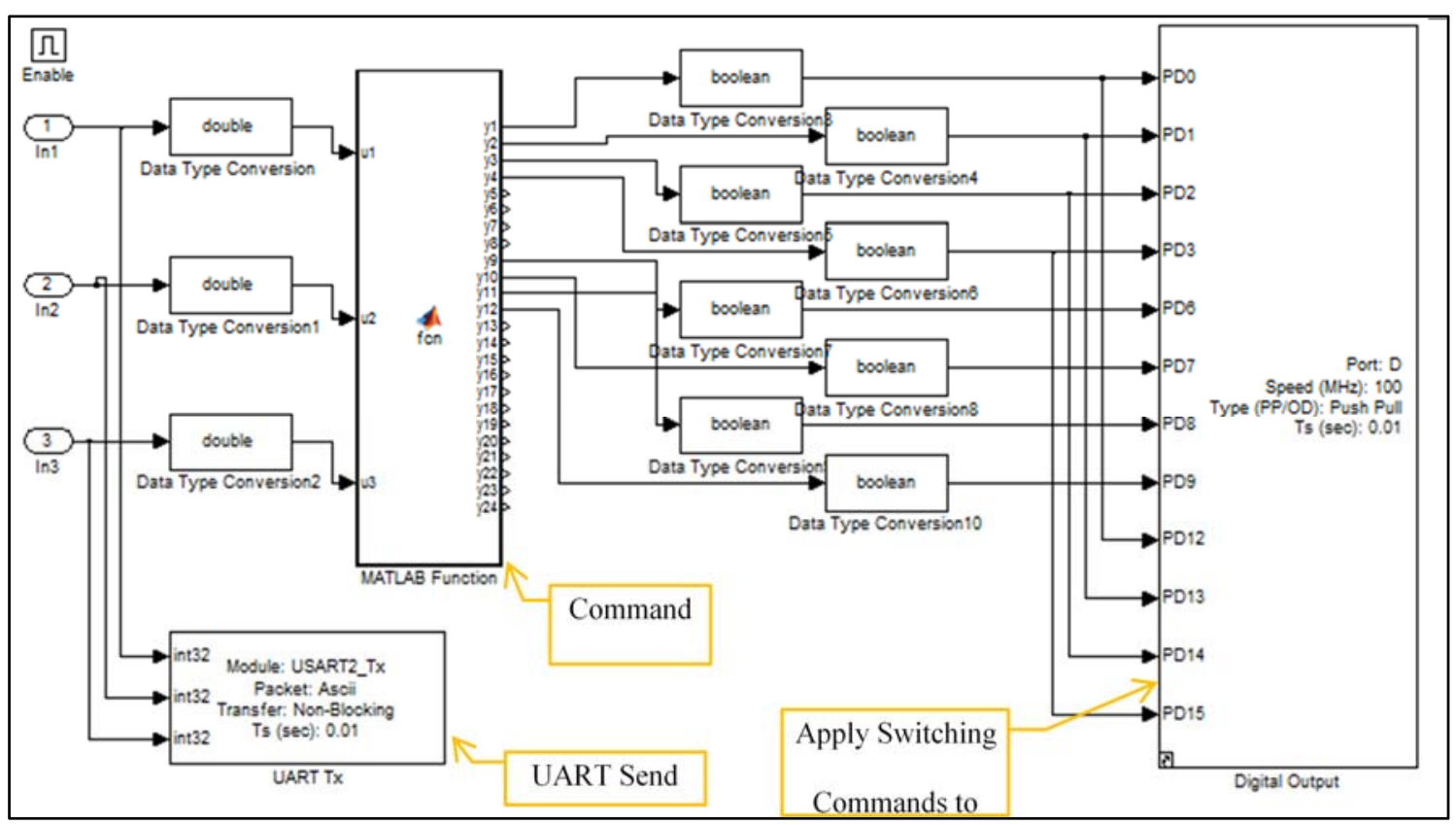

Figure 7.24 Subsystem in load control module.

In order to change the load, two software interfaces have been developed in the LabVIEW and finally released as an executable program. The first one is simple, and designed for testing. It has the capability of communication to STM32 through the UART port, and a total of 24 switches instantly. The front panel of the manual load change software is represented in Figure 7.25.
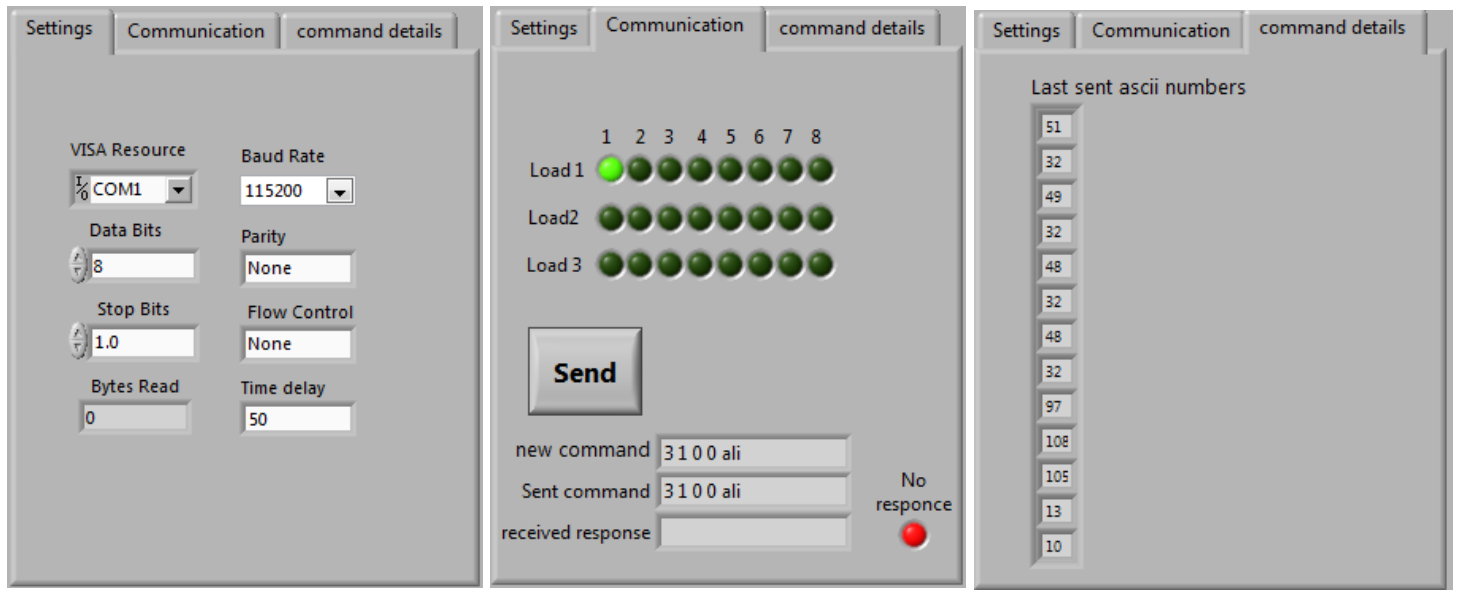

Figure 7.25 Manual load change software for inductive loads 
The second software is designed to have the programmability feature so it can work in a manual change or autonomous change mode. It is designed to be capable of applying any step load design, with any timing in an iterative loop, to any individual load.

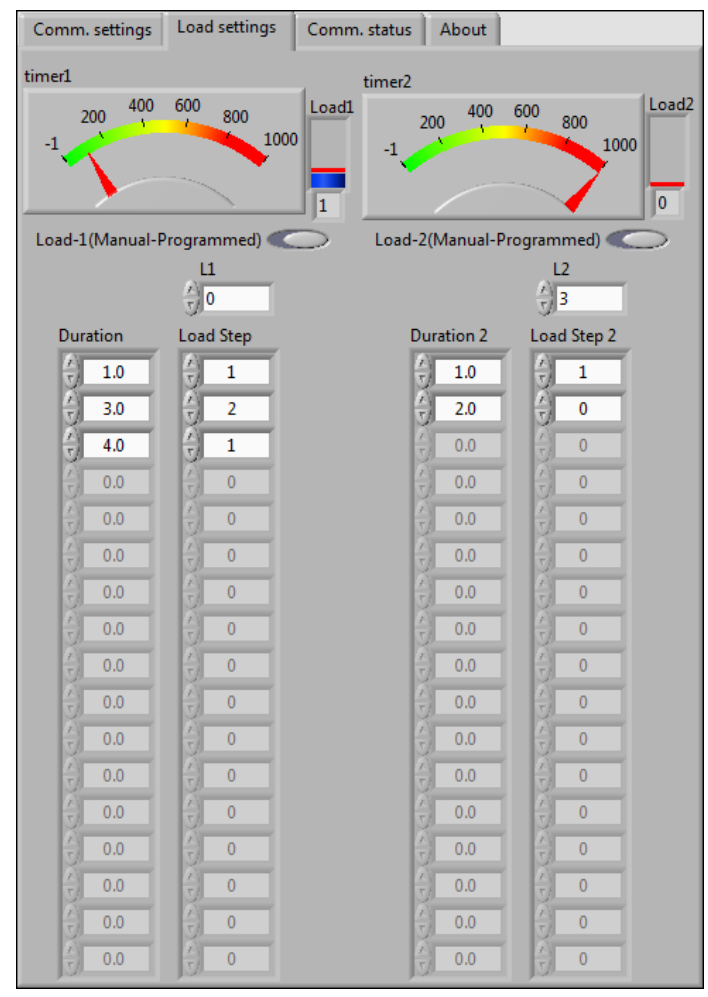

Figure 7.26 Programmable load interface

\subsection{Synchronizer Model}

In any $\mathrm{AC}$ power system, it is crucial to have synchronism at the time of closing the circuit breaker and at the Point of Common Connection (PCC). For this purpose, it is important to know that the status of the both sides of the breaker are known to be identical (minimum difference). The critical status parameters in the 3-phase power system are frequency, phase difference, sequence, and the voltage amplitude for each phase. Depending on the voltage level and utility requirements, every single parameter has a tolerance, which needs to be satisfied at the time of synchronism. 
Figure 7.28 shows the developed synchronizer, which is used in the testbed and the internal connection of the synchronizer module is available in Figure 7.27. It is equipped with the voltage measurements for 3-phases on both sides, in addition to the current measurements, as a complimentary, to be able to measure the transferred current and power.

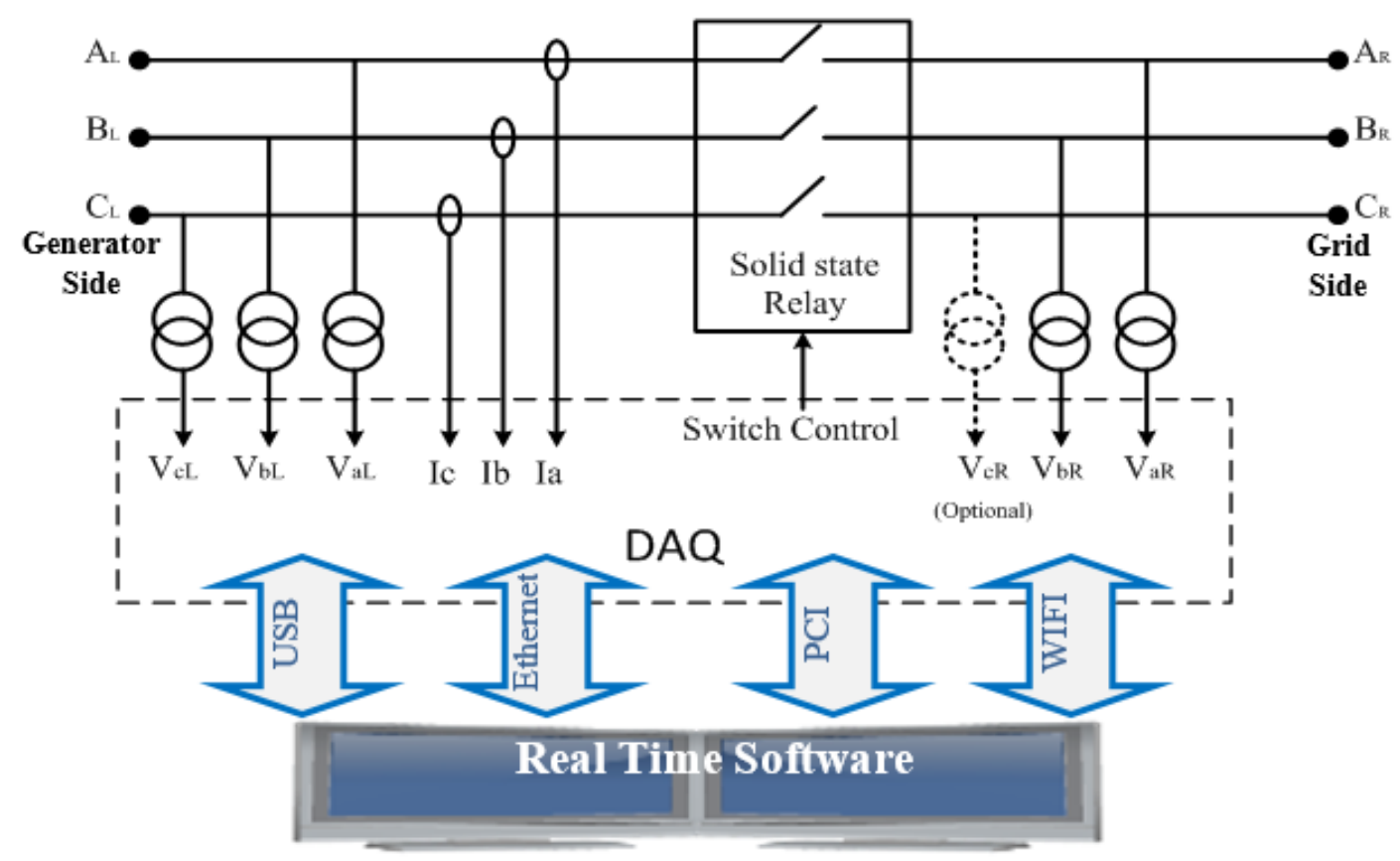

Figure 7.27 Internal connection of synchronizer module

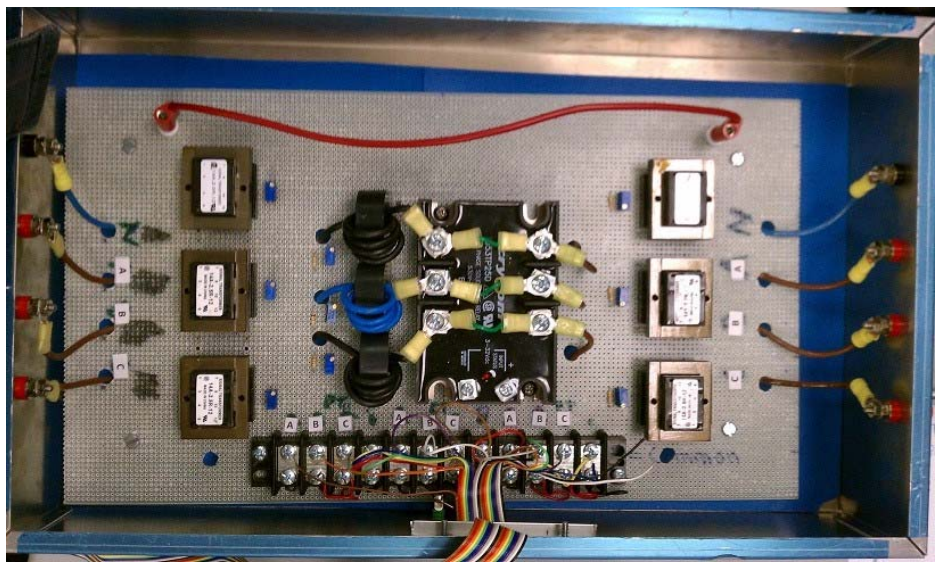

Figure 7.28 Developed synchronizer box 


\subsubsection{Automatic Synchronization}

Synchronization is always needed, when a new generating station joins the main power grid. Manual operation is not recommended, due to the reliability of the human operator and the possibility of huge damage that might have occurred, due to a wrong synchronization. Hence, an automatic synchronization algorithm was developed and practically implemented on the testbed. It uses a neighboring area and a very close condition area, depicted in Figure 7.29. In order for the synchronizer to send the close signal to the breaker, the conditions should be maintained in the neighboring area for a period of time continuously, and as soon it gets into a very close situation, the safe signal can be sent to the breaker.

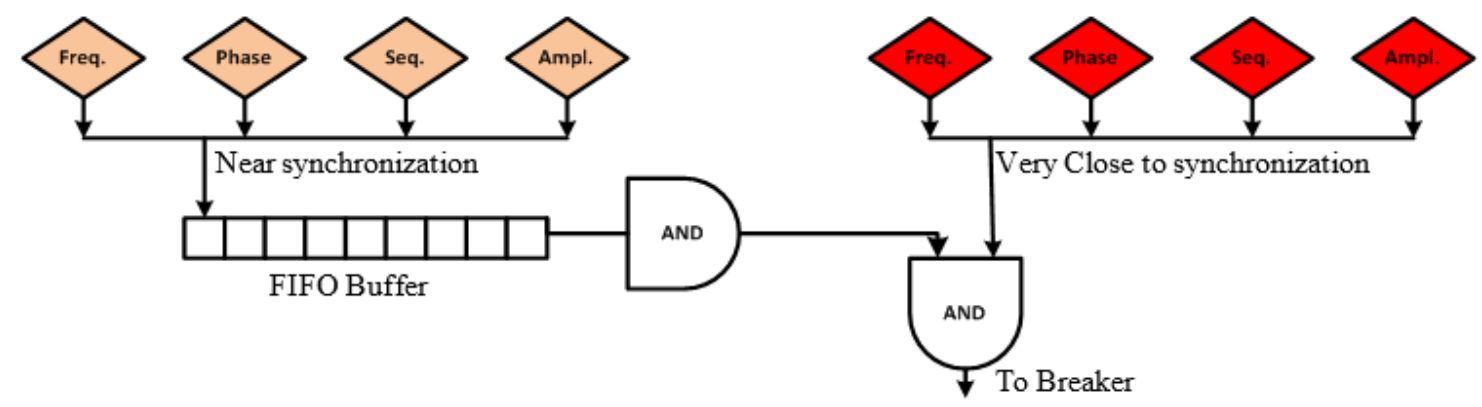

Figure 7.29 Automatic synchronization logic

\subsubsection{Dynamic Brake}

The Dynamic Brake device enhances the synchronization process for generating stations, when connecting to the power grid. For the testbed operation, this device can be used by several generating stations, using a multiplexing system depicted in Figure 7.30. The synchronization process and the related devices will be completely explained in chapter 7 . 


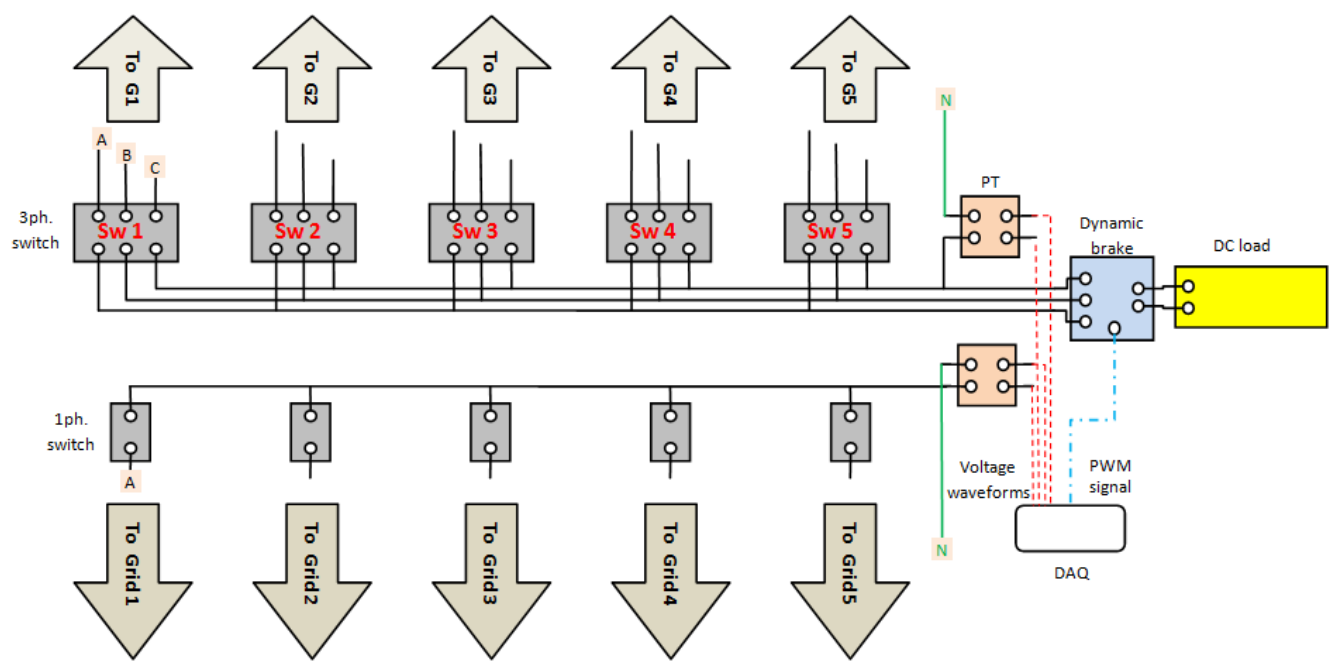

Figure 7.30 Multi-brake system 


\section{DEVELOPMENT OF HYBRID AC-DC POWER SYSTEMS INVOLVING RENEWABLE ENERGY RESOURCES}

The future power system will be highly relying on renewable energy resources. Correspondingly, the need for expert designers and operators for such system is increasing. This chapter includes the implementation of energy source emulators and related control attributes to prepare a suitable method for achieving appropriate solutions, a better understanding of the actual real world problems, and a better integration of renewable energy resources through experimental tests in an about to reality environment. First, the concept of renewable energy resources will be discussed in this chapter. Then, by evolving the new platform for design, development, and integration of renewable energy resources, such as photovoltaic and wind, the developed techniques for integrity of hybrid power system features for both research and educational aspects will be presented. This part of the research involves techniques, such as restructure of AC-DC power converters, real-time operation of them, as well as the analysis and the control. Altogether structure provides a state of the art platform to solve the most actual challenges of renewable resources integrated in modern power systems.

\subsection{Introduction}

During the past 20 years, energy costs have increased annually. In addition, the need for an efficient, less environmentally damaging, renewable energy resource are significantly growing. Generating electricity, from sources such as fossil fuels, biomass, and nuclear power plants requires a lot of water, which may intensify the drying of more 
lands and the warming of earth. However, renewable energy resources, such as wind and solar energy, can provide electricity with the lowest pollution and solve many of the problems leading to global warming and impacting energy demands in the near future. This issue is problematic especially for developed and industrial nations.

The objective of this chapter is to apply the concepts of integrating two main renewable energy resources into power grids with implementation examples in the laboratory-based testbed. The smart grid testbed developed at the energy systems research laboratory at Florida International University is introduced in the previous chapters. Hereby, the techniques for integrating and emulating renewable energy resources, such as wind and solar energy, the requirements, and the initiatives for performing experiments will be explained in the following sections.

\subsection{Implementation of Renewable Energy Resources}

Utilizing renewable energy resources will reduce the consumption of fossil resources for energy generation and transportation for the purpose of reducing carbon emission and air pollutants. Renewable energy resources also can be used to enhance the reliability, resiliency, and efficiency of microgrids. In the customer's side, resources for energy generations can cut the peak loads of customers and consequently the costs of the electricity. This will be improved, when the renewable energy resources are used along with the energy storage systems. It is worthy to mention that in the area of integration of renewable energy resources into interconnected power systems, energy storage systems are important enabling factor, due to uncertainty of such resources. The stored energy is used when it is needed. This can improve the transmission capacity, reduce the 
requirements for generation units, and maximize the use of variable resources, such as PV and wind. The negative aspects of energy storage systems are the losses, due to multiple conversions in $\mathrm{AC} / \mathrm{DC}$ or DC/AC stages, cost of energy storage devices, and the complexity of the conversion/operation process.

Large penetration of distributed generations and implementation of $\mathrm{HEV}$ into the grid provides a great opportunity by using idle battery storages of electric cars to be used as distributed energy storage systems.

From a technical point of view, the large implementation of variable resources will make the grid more complex to operate. Future power system engineers should consider such distributed generations and should be greatly familiar with technical aspects of it. Emulation of renewable energy resources in the testbed, not only provides an excellent opportunity for research, but also prepares the environment for testing the new ideas, in a highly technical research and development atmosphere, as well as providing a deep and realistic understanding in the field of renewable energy resources.

\subsection{Integrated Hybrid Power System}

\subsubsection{AC Power grid}

Traditional electrical energy systems are relied on the basis of AC network along with bulk energy generations. Distributed generations will be added to such systems through the microgrids. It is required to have the $\mathrm{AC}$ infrastructure as a basis for integrating any microgrids or for grid connected applications of renewable energy resources, and also to analyze their performance and evaluate the innovative ideas. In the emulated AC grid system, the cost, flexibility of operation, and safety (compared to 
actual high voltage systems) are the main advantages. The developed AC grid consists of generating stations, various programmable dynamic load modules, overhead transmission lines or cable models, synchronizers, AC measurement buses and feeders, in addition to wide area control, monitoring and operation package. This particular AC system has the flexibility to work in either 50 or 60 Hertz in 3-phase four wires connectivity basis [105].

\subsubsection{PV Emulation}

One of the main research topics studied in our hybrid AC/DC power system laboratory is development of a technique for implementation and energy management of large and small scale photovoltaic power plant. Photovoltaic arrays have a nonlinear voltage-current characteristic that its output power varies with solar radiation and cell temperature. Due to the limitation on solar energy availability in a laboratory environment, an alternative photovoltaic emulator is used. The emulator is a $6 \mathrm{~kW}$ Magna Power Electronics DC programmable power supply (Figure 8.1) and its maximum output voltage and current are $375 \mathrm{~V}$ and $15.9 \mathrm{~A}$, respectively.

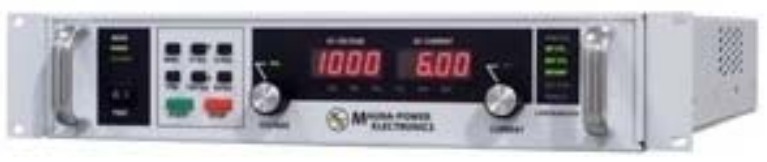

Figure 8.1 Programmable power supply

The Photovoltaic Power Profile Emulator (PPPE) software is used to calculate voltage and current profiles of a specific solar array based on predefined parameters (Figure 8.2). 


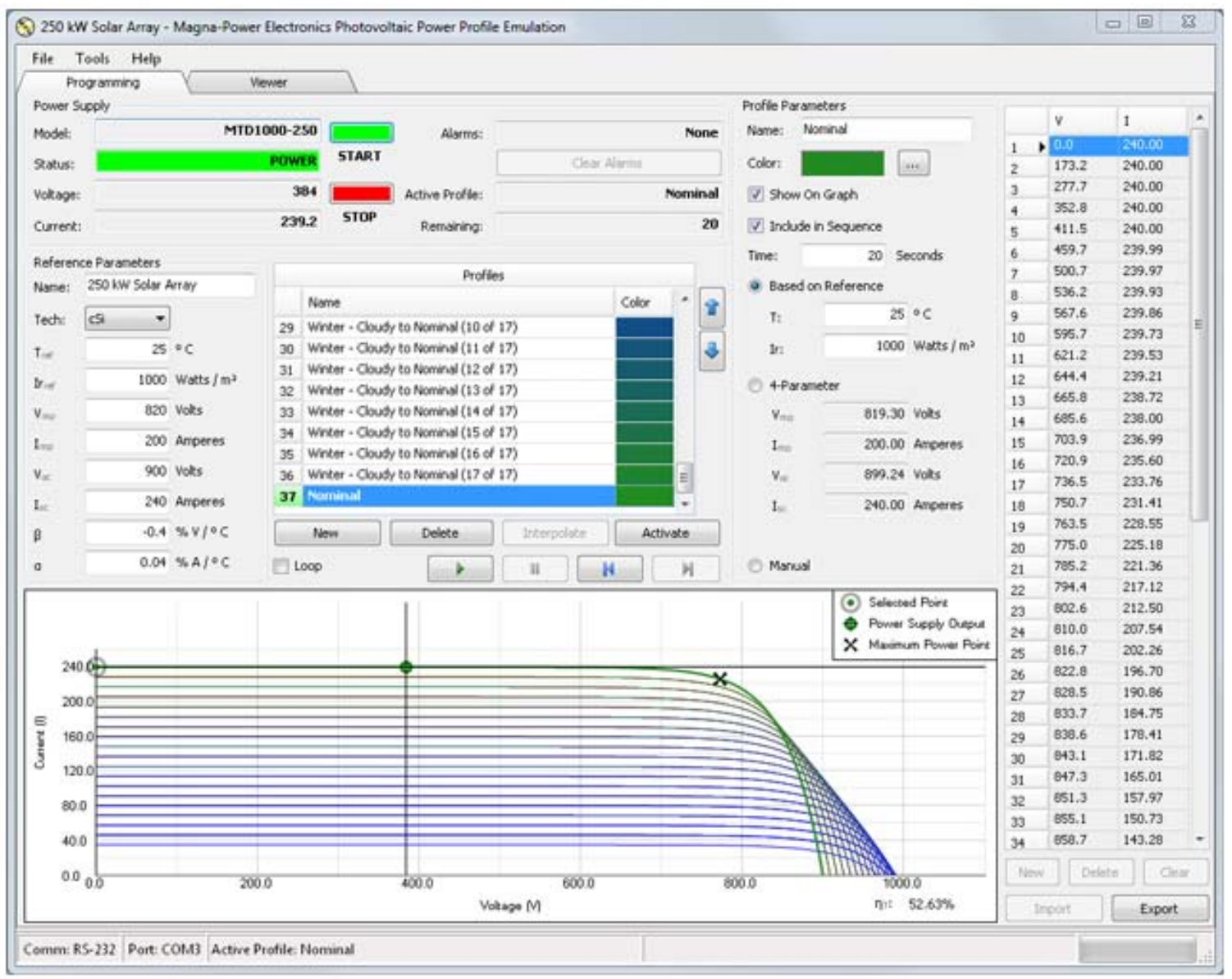

Figure 8.2 Photovoltaic power profile emulator software interface

These profiles can be sequentially sent to the power supply via RS232 serial communication interface. A power profile can be generated manually based on voltagecurrent curve of a photovoltaic cell or it can be defined based on the short circuit current, the open circuit voltage, and the Maximum Power Point (MPP) of a cell.

Figure 8.3 shows typical voltage-current and voltage-power characteristic of a PV module. As can be seen, the power curve has only a single maximum point and it is always desired that the PV module operate close to this point. Different maximum power point tracking (MPPT) techniques can be used to maintain the operation point at the MPP under varying conditions, such as load, temperature, and insolation. The MPPT 
algorithms can be classified into direct and indirect categories. The indirect methods are the "open-circuit voltage method", the "short-circuit method", the "look-up table method", and the "curve-fitting method". These methods are based on the data, which show the characteristics of the PV panel at different environmental and working conditions. The direct methods includes "artificial intelligence method", "differentiation method", "P\&Q method", and so on. The direct methods are more robust, i.e. prior knowledge of the PV parameters is not required. However, voltage or current measurements and a feedback loop are necessary for the converter's control circuit [105].
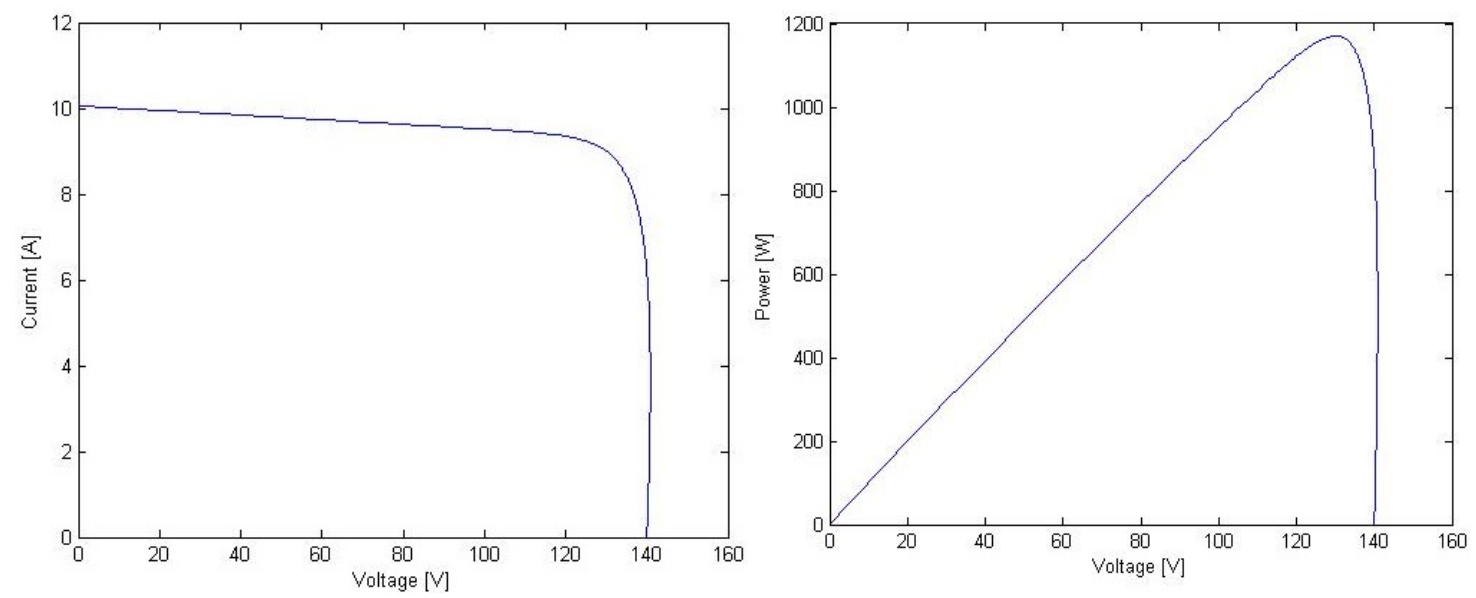

Figure 8.3. Voltage-current and voltage-power curve of a photovoltaic module.

Figure 8.4 shows the schematic diagram of photovoltaic system with MPPT controller. The PV module and the DC bus are interfaced with each other through a DC/DC converter, which can be a step-down or step-up depending on the DC bus voltage and the panel size. The controller circuit samples the voltage and the current at the output of the photovoltaic panel. Then the switching signal is sent to the converter to regulate the output current and to maximize the power transfer. 


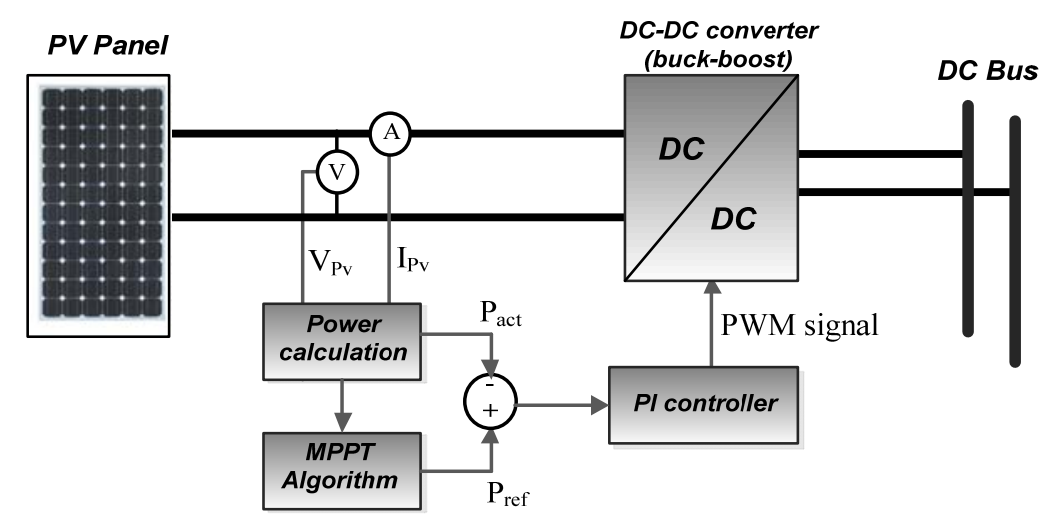

Figure 8.4. Block diagram of a photovoltaic system with MPPT controller

\subsubsection{Wind Turbine Emulator}

For wind turbines, induction or synchronous generators are often used to convert the kinetic energy of the wind into electric power. Permanent Magnet Synchronous Generators (PMSG) have good advantages, because of the stable and secure behavior during normal operation and no need of any additional DC supply for the excitation circuit. In this machine, for modeling the generator, the following equations was considered:

$$
\begin{aligned}
& \frac{d i_{d}}{d t}=\frac{1}{L_{d}}\left[u_{d}+p \omega_{g} L_{q} i_{q}-R_{d} i_{d}\right] \\
& \frac{d i_{q}}{d t}=\frac{1}{L_{q}}\left[u_{q}+p \omega_{g}\left(L_{d} i_{d}+M i_{f}\right)-R_{q} i_{q}\right]
\end{aligned}
$$

Where $i_{d}$ and $i_{q}$ are the stator currents, $u_{d}$ and $u_{q}$ are the stator voltages, $p$ is the number of pairs of poles, $L_{d}$ and $L_{q}$ are the stator inductances, $R_{d}$ and $R_{q}$ are the stator resistances, $M$ is the mutual inductance, and $i_{f}$ is the equivalent rotor current.

Due to wind speed variation, the output of the wind turbine is not constant; hence, a rectifier circuit should be utilized to convert the variable output $\mathrm{AC}$ voltage of the 
generator into constant DC voltage. The generated DC power can be employed in a stand-alone DC microgrid locally, or it can be converted into AC in order to be connected to the main AC grid. Figure 8.5 demonstrates the two configurations. In a DC grid connection, an isolated DC-DC convertor may be implemented to provide galvanic isolation for the wind turbine. Similarly, a transformer can be implemented for the AC grid connection to boost up the voltage level and isolate the wind farm from the utility grid as well.

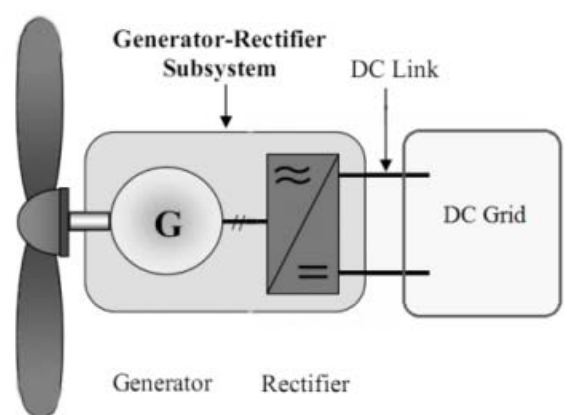

(a)

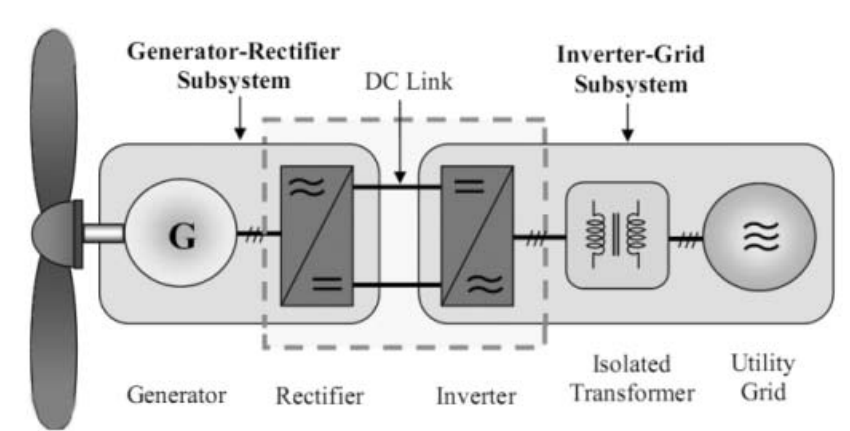

(b)

Figure 8.5. Wind generator electrical system: (a) DC grid connection (b) AC grid connection

The boost converter configuration is implemented, as an experimental setup, in our hybrid AC-DC power system laboratory (Figure 8.7) [106]. The wind turbine is imitated by a variable speed prime-mover and it is mechanically coupled with the selfexcited synchronous generator. A processor (DSP TMS320F240) on a digital platform was employed as the control heart of the system. For DC to AC inverter system, Space Vector Pulse Width Modulation (SVPWM) was employed with a $5-\mathrm{kHz}$ switching frequency.

The wind speed could be constant or varying in the form of pulses, sinusoidal, or a step change. Different patterns can be implemented on the DSP to emulate the wind 
turbine. In the real system, wind speed has a random variation according to the wind turbine location and its atmospheric conditions, but it can be set to operate within a given range speed variation. As an example, in order to see the unpredictable pattern and range of variations, Figure 8.9 shows the monthly average wind speed at La Venta station.

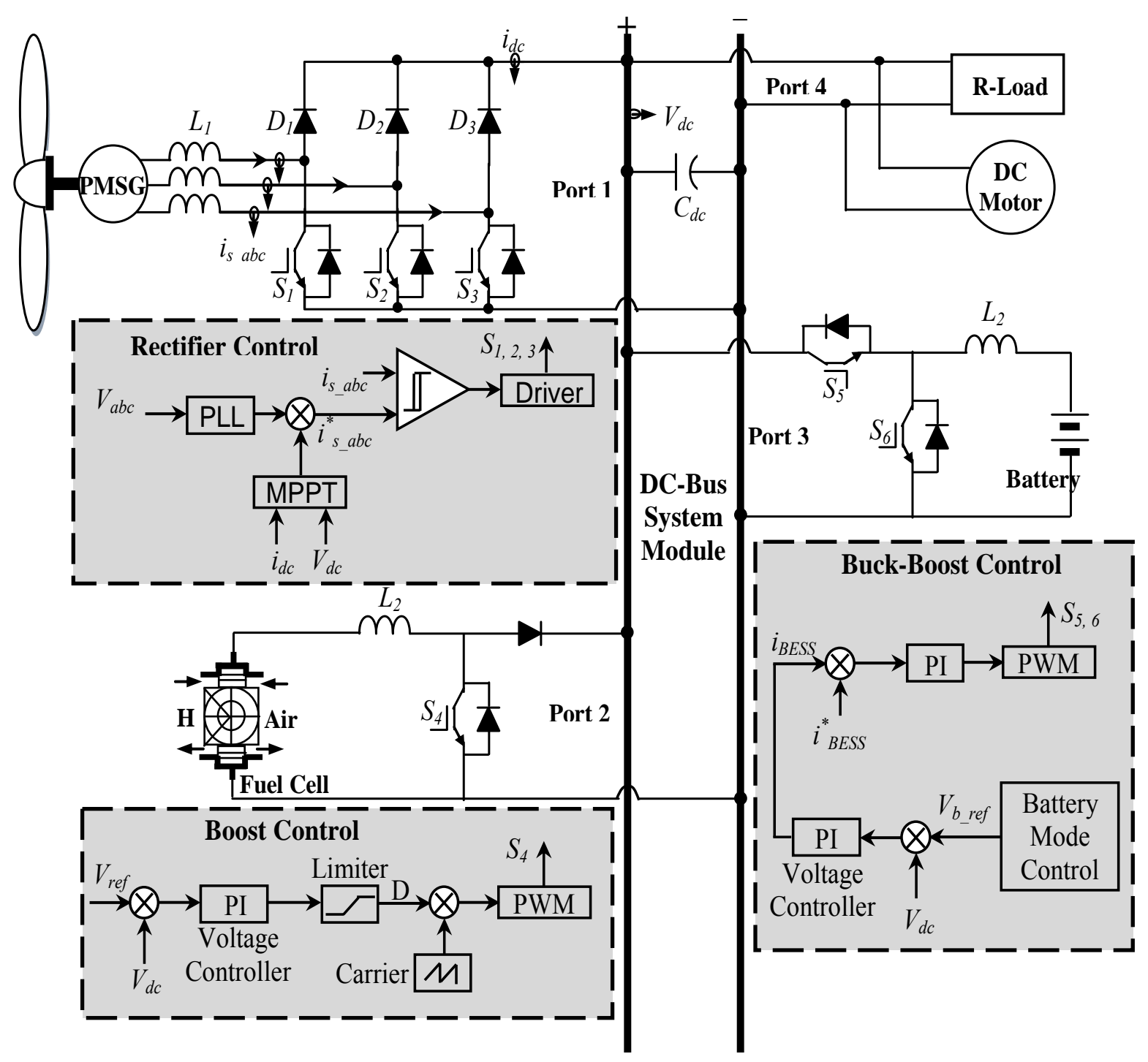

Figure 8.6. The overall DC system configuration 


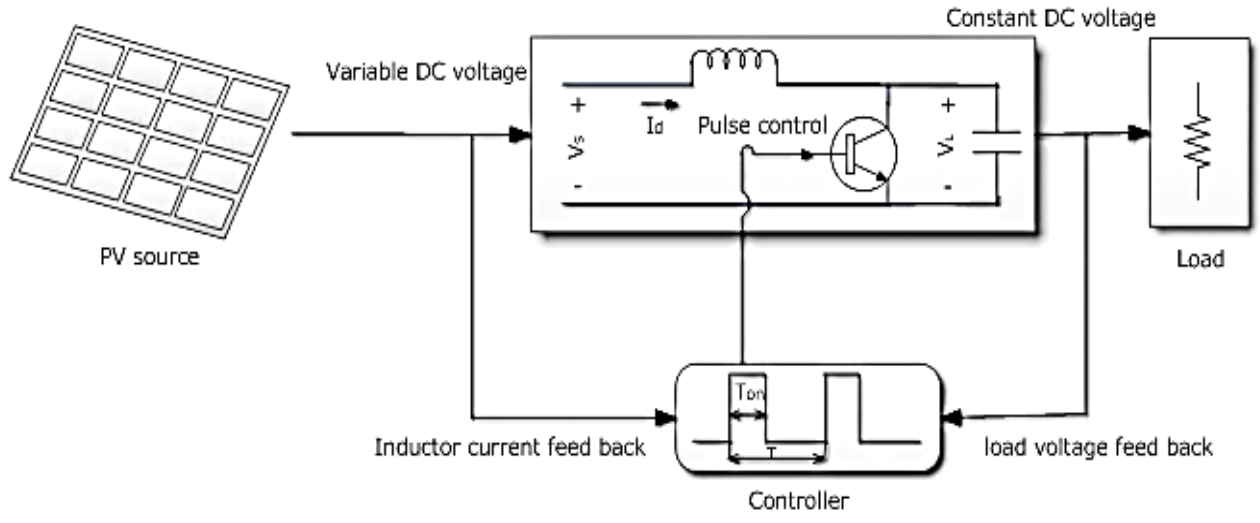

Figure 8.7. Boost converter circuit

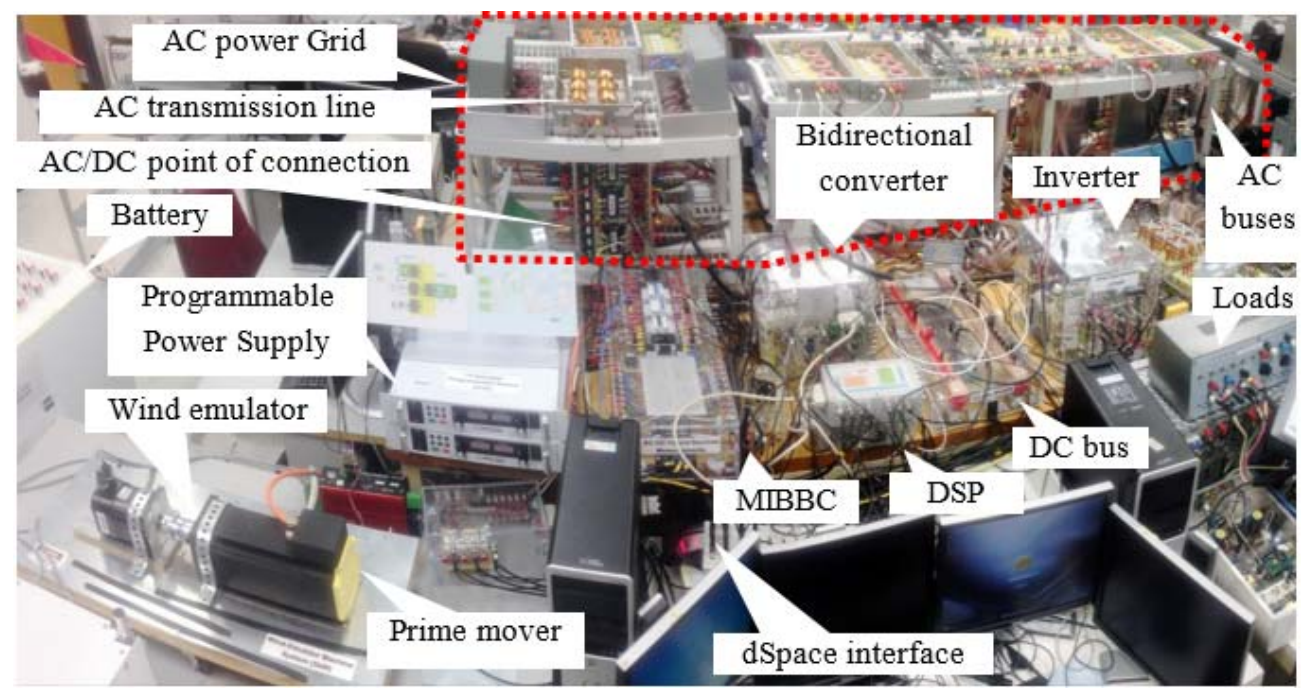

Figure 8.8. Experimental test setup for wind turbine and PV emulator.

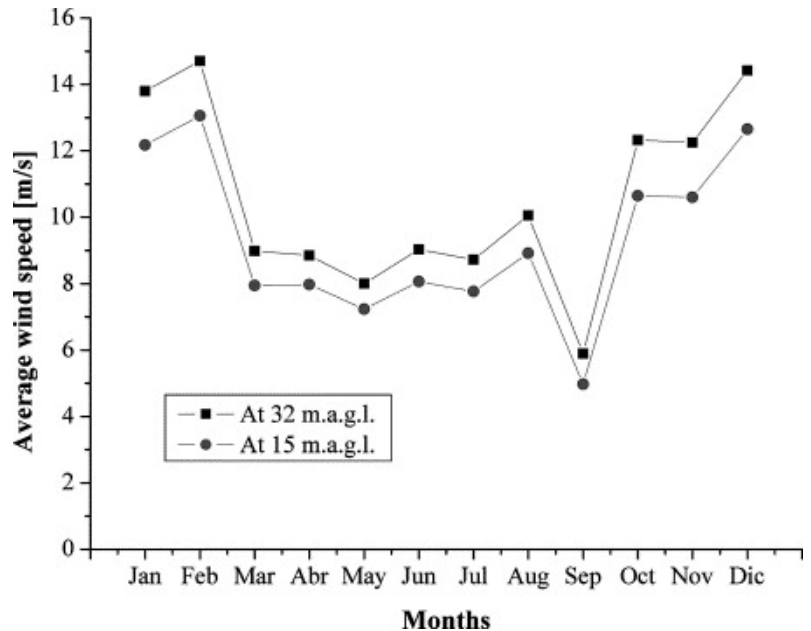

Figure 8.9. Monthly average wind speed at La Venta station [107] 
The wind test setup can provide a vision of the future power system and it offers the following research initiatives:

1) Integration of wind turbine and also the other energy resources like PV, fuel cell and diesel.

2) Efficiency improvement of power converters in wind turbine systems for battery charger and AC power.

3) Mathematical modeling and simulation of the power system under uncertainty

4) Installation of a small wind turbine and the grid connectivity

5) Design, fabrication, and test of the wind turbine systems using permanent magnet machines

6) Monitoring, diagnostics, and control of the wind generator

7) Impact of equipment aging and maintenance on wind turbines

8) Power system studies by considering different level of wind energy penetration

\subsubsection{Energy Storage System}

The energy storage systems considered in our system are the super capacitor bank and the battery bank.

\subsubsection{Battery Bank}

The battery bank includes twelve lead acid battery cells, which are rated $110 \mathrm{Ah}$ at 120 VDC. It provides additional power to the DC grid, when it is highly loaded, and in the cases where AC generators are not available (Out of service).

The battery bank is connected through a bidirectional Buck-Boost converter to the grid. During the charging period, the converter is in buck mode, and the duty cycle of the 
switching is controlled through a PI controller at a fixed frequency of 5-kHz. During the discharge period, another switch is activated and works in the boost mode.

\subsubsection{Super Capacitors}

The capacitor bank consists of 20 Maxwell's 16-VDC modules based on 350-F cells, which totally makes $2.9 \mathrm{~F}$. The maximum voltage the capacitor bank is about $340 \mathrm{VDC}$ and its continuous current is $12-\mathrm{ADC}$ at $\mathrm{T}=15^{\circ} \mathrm{C}$.

Considering the above mentioned values and the working condition of the capacitor bank, the total energy of this capacitor bank is:

$$
E=\frac{1}{2} C\left(V_{\max }^{2}-V_{\min }^{2}\right)
$$

This is because of the variable voltage of the capacitor bank. In cases of pulse loads, $V_{\max }$ is the voltage before the pulse load and $V_{\min }$ is after the pulse load. Considering the maximum acceptable voltage fluctuation on the DC bus, the available energy of the super capacitor bank will be limited consequently.

\subsection{Hybrid Grid Control and Operation}

\subsubsection{Isolated Microgrid Architecture and Operation}

Islanding is the situation when a portion of the power system, which includes generations and loads is isolated from the main system, but stays energized. The AC Microgrids, which turn into islanded status, usually cannot maintain their frequency. In islanded microgrids, the main issue is to satisfy the balance between the generation and the demand within the AC grid to keep the frequency unchanged. Since the islanding usually supposed to recover in a short time, utilization of storage systems will be very 
efficient way of keeping them working during the islanding situation. In this case, the battery will be charged, when generation is more than demand and the frequency likes to increase. On the other hand, discharging the batteries can provide the grid with the missed energy because of disconnection from the main grid. The first goal, after maintaining the islanded area under normal electrical situation, is to reconnect it to the main grid. For this purpose, same voltage characteristics are required at the both side of the reclosing breaker. Although synchronizers will make the decision on ideality situation of reconnecting, however the microgrid status should be controlled to reach to the main grid, and this will be done by the help of generation and storage systems [108].

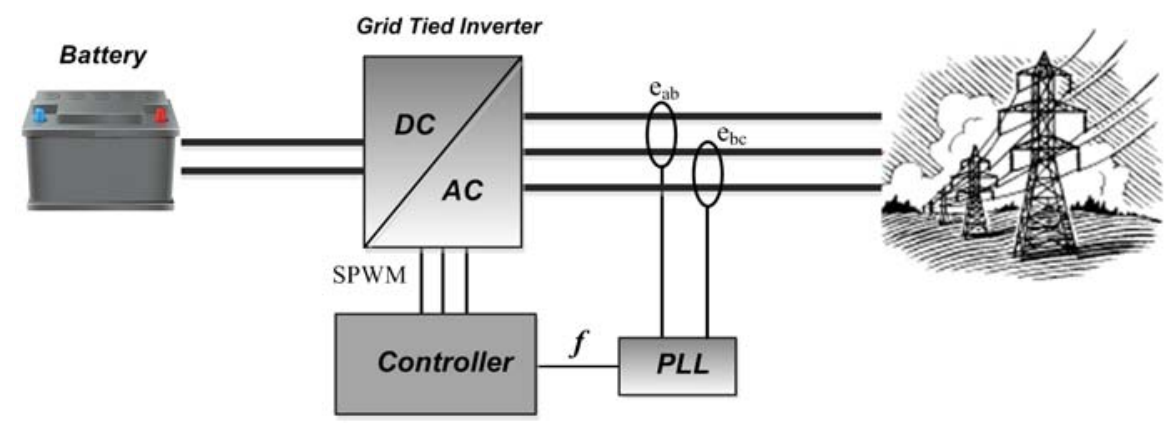

Figure 8.10. Battery energy storage for frequency regulation in islanded power system

\subsubsection{Developing Procedures for Power Balancing in DC Grid}

An interconnected AC-DC microgrid is using the rectifier circuit to maintain the voltage criterion within the boundaries. Particularly, a controllable type of rectifier is used for such a purpose. In this case, the renewable sources with DC connectivity will participate in feeding the loads, through current or power control mode of their operation. Depending on how fast they can react to a new reference command, they have different priorities in balancing the generation and demand response [109]. 
Unintentional islanding of microgrids will lose the voltage stability due to the absence of voltage control parameters. To keep the system stable, one of the units should take action fast and with a high reliability. It is recommended to use storage systems for this purpose [109]. In case of current injection from DC grid into AC grid, the story is totally different. The charging limit of battery storage systems is usually smaller than the discharge limit due to power electronic limitations [110]. The system should have enough inertia in order to delay the voltage rise and this inertia is provided by capacitor banks and DC capacitive characteristic of the DC grid.

\subsubsection{Bidirectional Energy Conversion in DC Microgrid}

There are many applications in DC power systems that need energy transfer in both directions i.e. between two or more DC buses or sources. For instance, in an electric vehicle, the power should be transferred from battery to the electric motor in acceleration mode of operation; on the contrary, in deceleration condition, the energy of the wheels should be sent back into the battery, which is called regenerative braking. In this mode, the electric motor will act as a generator and the battery will receive and store the energy. Both modes of operation are shown in Figure 8.12.

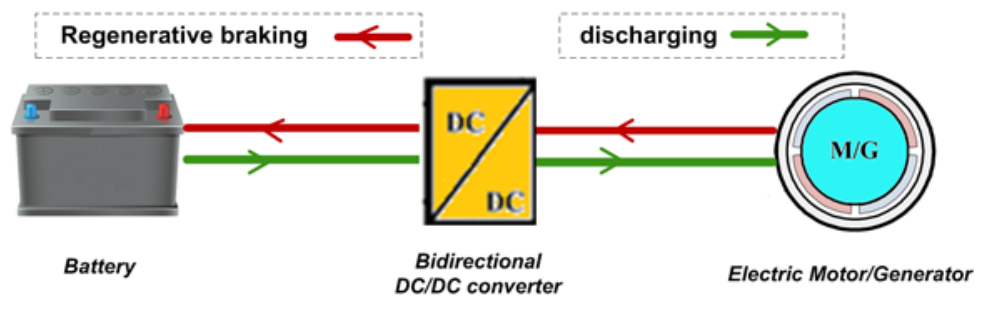

Figure 8.11. Energy transfer in electric vehicle

Moreover, several systems are currently employing DC distribution networks by now. For instance, in systems that require a high reliability and are involving a big 
number of electronic loads, such as data centers, DC network provides a more efficient solution for electric power distribution. Further applications of DC distribution systems include telecommunication, traction and spacecraft power systems, etc. Figure 8.12 shows the schematic diagram for the connection of two DC buses through bidirectional DC-DC converter.

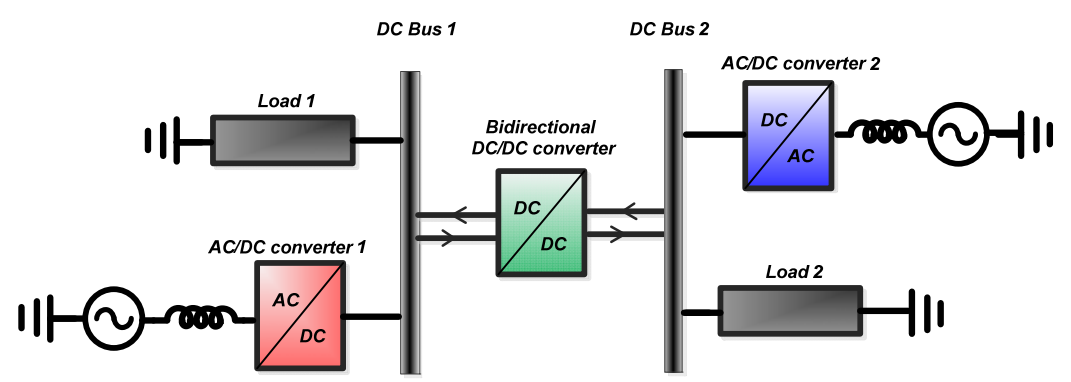

Figure 8.12. Schematic diagram of bidirectional energy transfer between two DC buses

Figure 8.13 shows the topology of bidirectional buck-boost convertor, which is implemented in our hybrid AC-DC power system. This convertor basically has two modes of operation:

i. In the boost operation mode, power is transferred from the low voltage terminal (LV) to the high voltage terminal (HV). In this mode, the first switch, S1, is turned off while the second switch is controlled by a PI controller in order to regulate the energy transfer. The steady state voltage and current can be found by:

$$
\begin{aligned}
& V_{H V}=\frac{V_{L V}}{1-\delta} \\
& I_{H V}=\frac{V_{S L V} /(1-\delta)-V_{S H V}}{R_{H V}+R_{L V} /(1-\delta)^{2}}
\end{aligned}
$$


Where, the $\delta$, $\mathrm{R}_{\mathrm{HV}}$ and $R_{L V}$ are the duty cycle, the equivalent resistor of the HV source and the equivalent resistor of the LV source respectively. Also, $V_{L V}, V_{S L V}$ and $V_{S H V}$ are the LV terminal voltage, the equivalent voltage of the source connected to the LV side and the equivalent voltage of the source connected to the HV side respectively.

ii. In the buck operation mode, power from HV terminal is transferred to the LV terminal. In this mode, the PI controller regulates the power by adjusting the switching cycle of the S1 continually. A necessary condition for buck operation mode is that the $\delta V_{S H V}$ should be higher than the $V_{S L V}$. During this mode, the voltage and the current of the low voltage terminal can be given by (8.6) and (8.7) respectively.

$$
\begin{aligned}
& V_{L V}=k V_{H V} \\
& I_{L V}=-\frac{k V_{S H V}-V_{S L V}}{k^{2} R_{H V}+R_{L V}}
\end{aligned}
$$
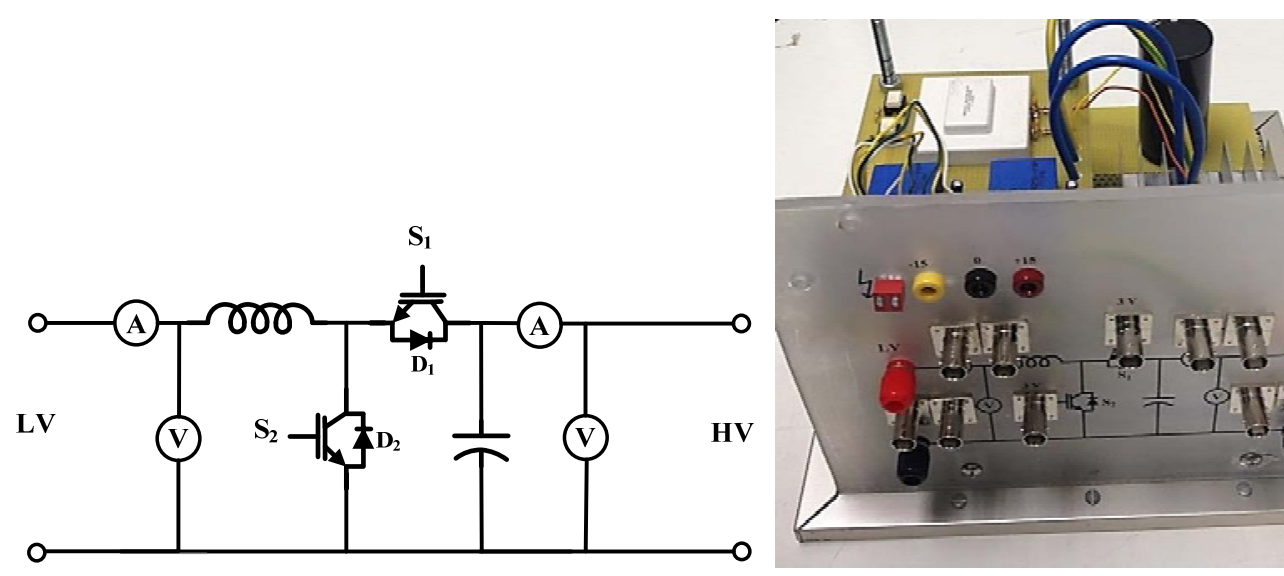

Figure 8.13. Bidirectional buck boost convertor schematic and actual developed device 
In the buck mode, power from HV will be transferred to the LV. In this mode the second switch, S2, will work as a diode and the first switch, S1, will be controlled by a PI controller and a PWM in order to control the energy flow.

The inductor and capacitor used in the converter are $2.7 \mathrm{mH}$ and $1200 \mu \mathrm{F}$ respectively. The maximum input current into the LV side is $12 \mathrm{~A}$ and it is mainly limited because of the inductor capacity. For control and monitoring purposes, two voltmeters and two ammeters were connected to measure the current and voltage at the two terminals. In practice, for bidirectional energy flow, the DC-DC converters are usually operated in current control mode. In this case, one of the ammeters may be enough for control of the current. However, the other meters can be used for monitoring purposes.

There are many issues attached with bidirectional DC-DC convertor. To realize the benefits of bidirectional energy transfer, these converters must be capable of regulating of their output voltages at a different load, while maintaining a high efficiency. Also, there are connectivity issues, linking two different DC buses, whose voltages are at different levels and contain significantly different content of ripple.

\subsubsection{Bidirectional power flow in the interconnected AC/DC grid for controlling the active and reactive power flow}

The high penetration of renewable energy sources, yielding DC output, and also the increased number of electronic loads and machine drives operating with DC input, made DC microgrids very important. In case that the generation in a DC microgrid is not balanced, the excess power can be stored during low consumption intervals and be available during high demand intervals. Also, a grid-tied inverter can be implemented to provide bidirectional power flow between the DC microgrid and the existing AC grid. In 
this case, any excess DC power can be sent to the transmission grid, and also power from the AC grid can be drawn to make up for any power shortfalls in the DC microgrid.

While the storage system is an advantage in areas, which are disposed to power outages, grid-tied inverter systems are much more cost-effective. This is because of the grid-tied inverter operating using fewer components, as well as the sizing of the system is not dependent on the electrical load. Nonetheless, compound system of the battery storage and the grid-tied inverter can provide both advantages with proper sizing.

Bidirectional grid-tied inverter test setup is implemented in our hybrid AC-DC power system laboratory. A vector decoupling control technique with Sinusoidal Pulse Width Modulation (SPWM) is implemented to regulate the active and reactive power flow between the DC and AC grid. Figure 8.14 shows the circuit diagram of the system and its control elements. The dynamic model of this system in the state space can be represented by (8.8).

$$
\left[\begin{array}{c}
\frac{d i_{q}}{d t} \\
\frac{d i_{d}}{d t} \\
\frac{d v_{d c}}{d t}
\end{array}\right]=\left[\begin{array}{ccc}
\frac{-R}{L} & \omega & \frac{-s_{q}}{L} \\
\omega & \frac{-R}{L} & \frac{-s_{d}}{L} \\
\frac{3 s_{q}}{2 C} & 0 & \frac{-1}{C R_{L}}
\end{array}\right]\left[\begin{array}{c}
i_{q} \\
i_{d} \\
v_{d c}
\end{array}\right]+\left[\begin{array}{ccc}
\frac{-1}{L} & 0 & 0 \\
0 & 0 & 0 \\
0 & 0 & 0
\end{array}\right]\left[\begin{array}{c}
e_{q} \\
0 \\
0
\end{array}\right]
$$

Vector decoupling technique is used for autonomous control of the active and reactive power flow. The controller is built base on the state space model of the system and the equations are given by (8.9) and (8.10).

$$
v_{r q}^{c o n t}=\omega L i_{d}+e_{q}+k_{p}\left(i_{q}^{r e f}-i_{q}\right)+k_{i} \int\left(i_{q}^{r e f}-i_{q}\right) d t
$$




$$
v_{r d}^{\text {cont }}=-\omega \operatorname{Liq}+k_{p}\left(i_{d}^{r e f}-i_{d}\right)+k_{i} \int\left(i_{d}^{r e f}-i_{d}\right) d t
$$

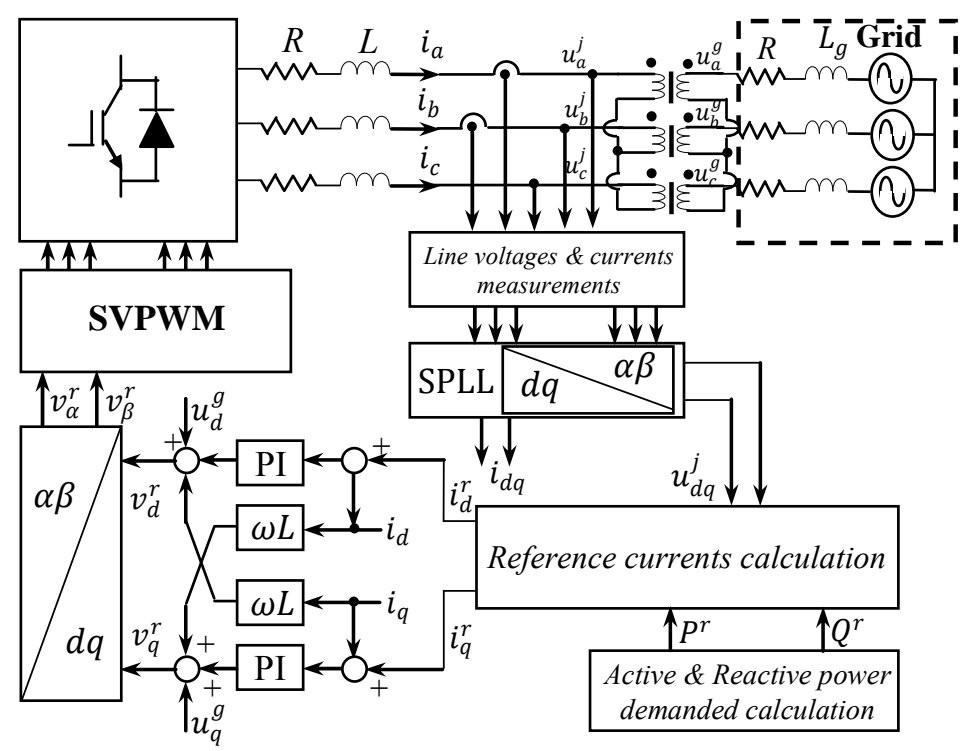

Figure 8.14. Vector decoupling control of the bidirectional converter for power sharing of hybrid systems

The controller can work at unity power factor if the reference value of $i_{d}$ is set to zero. The grid-tied inverter is operating at $8 \mathrm{kHz}$ switching frequency and the sampling time of $0.3 \mathrm{~ms}$, which allows fast response of the system to different load demands at either the AC or DC sides. Corresponding active and reactive power flows can be driven from equations (8.11) and (8.12).

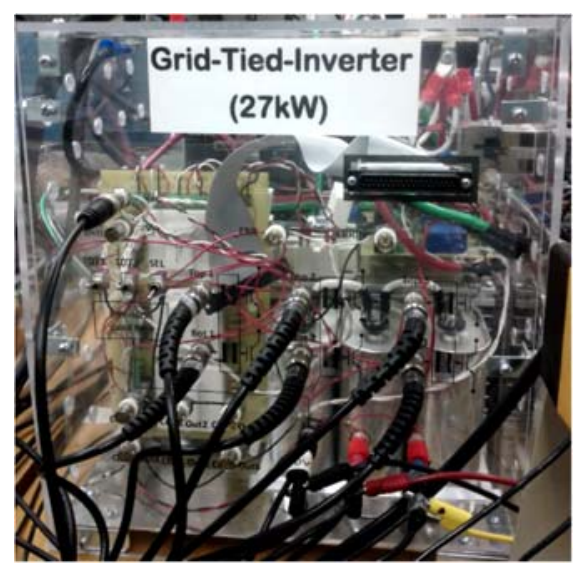

Figure 8.15 Developed grid-tied inverter in energy systems research laboratory 


$$
\begin{aligned}
& P(t)=\frac{3}{2}\left[u_{d}^{j}(t) \cdot i_{d}(t)+u_{q}^{j}(t) \cdot i_{q}(t)\right] \\
& Q(t)=\frac{3}{2}\left[u_{q}^{j}(t) \cdot i_{d}(t)-u_{d}^{j}(t) \cdot i_{q}(t)\right]
\end{aligned}
$$

\subsection{Educational Aspects of the Developed Platform}

For educational purposes, this material can be valuable for students majored in energy systems. In addition, the complementary course involves students in practical techniques, which are not available in theoretical course work coverage. The developed system can also be used to implement protection and control ideas associated with new developments in the field of smart grid. There are several experiments, which were designed for the students' involvement according to practical aspects of hybrid energy systems. These experiments are listed as the following:

1) Designing and speed control of wind emulator machine system

2) Power sharing control of hybrid AC/DC systems

3) Bidirectional energy conversion of power sources in DC microgrid systems

4) Designing and building of transmission line models and AC bus model

5) Wide area measurement and operation of the AC grid

6) Bidirectional power flow between $\mathrm{AC}$ and $\mathrm{DC}$ systems using grid-tied inverters 


\subsection{Conclusion}

Implementation of hardware and software components allows the evaluation of different techniques and scenarios in operation of power grids involving automation, control, monitoring, and analysis in presence of renewable resource. A test setup was developed by the research group to be used as an educational platform to simulate most common types of renewable energy resources in the modern power systems.

Integration of such renewable resources are introduced for realistic techniques which including wind turbine and generator system behavior as well as the speed control of motor-generator sets through the inverter-drive electronics. They use power sharing control program using Matlab/Simulink for design and control purposes. The control of the DC bus voltage and the evaluation of the bidirectional DC-DC convertors performance are needed to be considered in the design. For the distributed generation and the demand side management advanced power electronics techniques was utilized. Applications such integrated systems, for secure operation of islanded microgrids, power sharing, renewable energy resources utilization, etc. along with experimental verifications, will be addressed in the next chapter. 


\section{PHASOR MEASUREMENT UNITS IN SMART GRID}

\subsection{Introduction}

A new wave of regulations and business models were made recently for power systems, and generating companies are providing megawatts of energy through interconnected networks and delivering energy to various customers from distributed generator companies. The expectations for a safe, reliable, and economical operation of this broad scope electric power system require advanced Wide-Area Monitoring, Protection and Control (WAMPAC) capabilities and applications. PMUs are the devices that provide time-synchronized coherent data from the entire network. They enable the complete system status to be observable and controllable in real-time. Traditionally, most of the efforts have shown WAMPAC systems in the transmission network for various applications, such as transient stability [111], modal estimation [112], and load shedding [25]. More recently, it was realized that PMUs represent a useful contribution for challenging operation of active distribution networks [113], [114]. Fault location detection, islanding detection, state estimation, and distributed generation oscillation monitoring are some of the synchrophasor applications in active distribution networks. As the necessity of PMU deployment on both distribution and transmission networks increases, the intent to establish practical small-scale PMU grows. The development of a reliable PMU setup is a challenging issue, however, it is a subject of increasing interest by many research groups. In [115], the authors reported a laboratory-based PMU development and implementation in the Danish power grid. Distributed state estimators, 
protective relaying under transient conditions, and interoperability of a test setup have been addressed in [116], [117]. The hardware and software test platforms are also reported, in order to examine capabilities of PMUs [118]-[121]. In this case, the LabVIEW environment was used to demonstrate applications accompanied with a realtime digital simulator and an actual testbed [122], [123].

The high cost of traditional actual PMU devices inhibits industry and utilities to expand further deployments. To overcome this growing expensive bottleneck, in this dissertation, we propose a practical laboratory based PMU development by using a data acquisition (DAQ) device and the global positioning system (GPS). The system uses an actual GPS receiver to provide the timing signal (IRIG-B), then decodes the signal, and retrieves the trigger instance and the corresponding time information. An off-the-shelf PDC is used to collect time-stamped data and sort them in a comparable way to establish high-precision observable system status. The steady-state and dynamic performance of the proposed setup is compared with already validated PMU devices from SEL. Realtime simulators and software-based testing platforms may overlook the actual power system's component behaviors. Therefore, this dissertation also presents a real-time experiment on the smart grid testbed available at Florida International University. This chapter is organized as the following. In 9.2, PMU, timing-requirements, and C37.118 synchrophasor protocol are introduced. Section 9.3 explains the structure and the development of the proposed PMU. Section 9.4 presents the evaluations and the performance comparison side by side to a commercial PMU in a PDC environment. The laboratory setup for the real-time experiment is presented in Section 9.5. Finally, Section 9.6 provides the conclusion and discussions on this topic. 


\subsection{Definitions and the Structure}

\subsubsection{Synchronized Measurement in Power Systems}

PMUs are widely used as synchronized measurement devices for the power system applications. Their measurements are obtained from broadly distributed locations, and synchronized with respect to a global and precise time reference, which is GPS. The technology for developing the PMUs is rapidly growing and many vendors are offering them with additional integrated peripherals, such as protective relaying. Standalone PMUs are increasing around the world and are mostly installed for the research purposes. Their application in real-time Wide Area Monitoring, Protection and Control (WAMPAC) systems enables the full utilization of the potential of the power system. It has a great value of synchronizing the measurements and controls. Therefore, there has been significant research work and many developments related to time-synchronized measurements in power systems.

\subsubsection{Global Time Reference and PMU basics}

The synchrophasor standards [124] and [125] define the necessary technical requirements of synchrophasor measurements and data transfer schemes [126]. In order to enable a universal PMU prototype, in this dissertation, the defined requirements are strictly followed.

The phasor representation of a sinusoidal waveform in AC power system applications is usually presented as (9.1):

$$
X(t)=X_{m} \cos (\omega t+\phi)=X_{m} \cos \left(2 \pi f_{0} t+\phi\right)
$$


where $X_{m}$ is the signal magnitude, $f_{0}$ is the nominal frequency, $\omega$ is the angular frequency, and $\phi$ is the instantaneous phase angle relative to the cosine function at the nominal system frequency synchronized to Universal Time Clock (UTC).

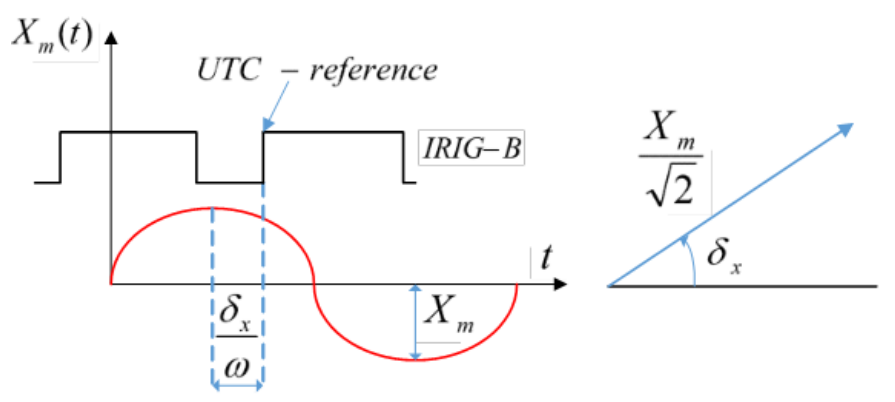

Figure 9.1 Time stamping

In general, the magnitude and the frequency can be a function of time; however in special cases, where $X_{m}$ is constant, the frequency has a constant offset from the nominal frequency:

$$
\begin{aligned}
& \left.X(t)=X_{m}(t) \cos \left(2 \pi f_{0} t+\left(2 \pi \int g d_{t}+\phi\right)\right)\right) \\
& g=f-f_{0}=\Delta f
\end{aligned}
$$

For a series of measurements with a $T_{0}$ period, for each measurement sample, the phase shift is:

$$
\phi_{T o}=2 \pi\left(f-f_{0}\right) T_{0}
$$

\subsubsection{Total Vector Error}

IEEE C37.118 defines the criterion to evaluate the accuracy and the error in phasor measurements. The error is defined by the difference between the actual and the measured phasors. 


$$
\vec{V}_{\text {error }}=\vec{V}_{\text {meas }}-\vec{V}_{\text {actual }}
$$

Where $\vec{V}_{\text {error }}$ is the error phasor, $\vec{V}_{\text {meas }}$ is the measured phasor, and $\vec{V}_{\text {actual }}$ is the actual phasor of the voltages/currents.

Total Vector Error (TVE) is a criterion to express the magnitude, the phase, and the timing error for synchrophasor measurements. TVE is represented in percent:

$$
T V E=\frac{\left|\vec{V}_{\text {meas }}-\vec{V}_{\text {actual }}\right|}{\left|\vec{V}_{\text {actual }}\right|} \% 100
$$

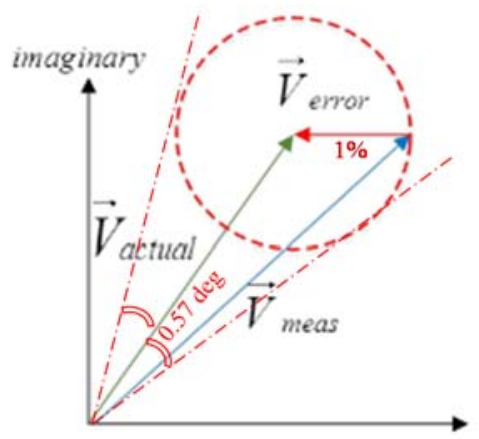

Figure 9.2 Total Vector Error

The design of the system is subjected to be a tradeoff between the accuracy and the cost. Therefore, it is not recommended to exceed the requirements of accuracy by neglecting the cost. The maximum allowed TVE is $1 \%$, according to the standards' requirement.

\subsubsection{Time Format}

The Inter-Range Instrumentation Group (IRIG) is an accurate standard format and is described in standard 200.04 [127]. The IRIG-B standard, which is adopted by electric utility applications, has information of second, minute, hour, day, year, and optional 
control bits. The time information, which contains 100 pulses, is reported with a rate of 1 message per second. The time information signal can be in TTL (DC-pulse width) format, which is unmodulated and without a carrier signal. Alternatively, an amplitude modulation can be applied with a sinusoidal carrier signal.

\subsubsection{Timing Requirements}

A PMU should be capable of receiving a reliable and accurate time over a broad geographic zones. The GPS can provide the UTC with a sufficient accuracy to keep the TVE, Frequency Error (FE), and Rate of Change of Frequency (ROCOF) within the acceptable limits. In a $60 \mathrm{~Hz}$ system, 1 micro second represents 0.022 degrees. $1 \%$ TVE corresponds to a 26 micro-second error. It is recommended to have the time reference at least 10 times more accurate than the above limit.

\subsubsection{Reporting Latency}

The latency is an important factor for evaluating the performance of PMU and PDC. The latency is assumed to be the maximum time interval between the time-stamped data and the moment when the data becomes available at PMU output, expressing the first bit of data. The latency is dependent on various factors, such as sampling window, calculation/estimation method, PMU processing time, and reporting interval.

\subsubsection{Message Reporting}

All phasor measurements, including frequency and ROCOF, should be reported at a constant rate, which is a positive integer number per second. The reporting rate (frames per second) can be adjusted according to the requirements. In this study, we use 1 frame per second, but the hardware is designed to carry out 10 messages per second as well. 


\subsubsection{Data Frame and PDC Reporting}

A PDC is considered to be a node in communication network, which collects the measurements from different PMUs or other PDCs, correlates the data by time tag and creates an awareness system from wide-area measurements. A PDC can also provide quality check on phasor data, disturbance flag check, achieved progress, result displaying, and a SCADA interface.

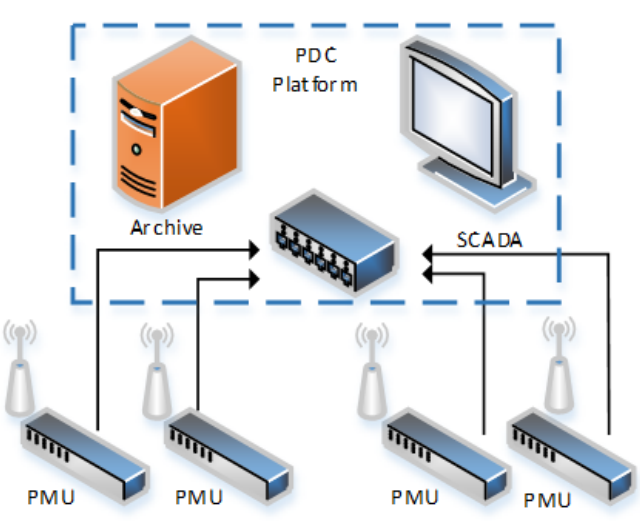

Figure 9.3 PDC platform

The PMU information is transferred to PDC by data-frames. Four types of frames are defined in the synchrophasor messaging standard:

- Data frame

- Configuration frame

- Header frame

- Command frame

The first 3 frames are transferred from the data sources (PMU or PDC) and the last one is created only by the reporting station. The frames contain actual message information. The common word definitions for all frame types are frame synchronization word (SYNC), frame size word (FRAMESIZE), data stream ID number (IDCODE), 
second-of-century (SOC), fraction of second and time quality (FRACSEC), data word (DATA n), and cyclic redundancy check (CHK). Other word definitions can be obtained from the synchrophasor protocol [128].

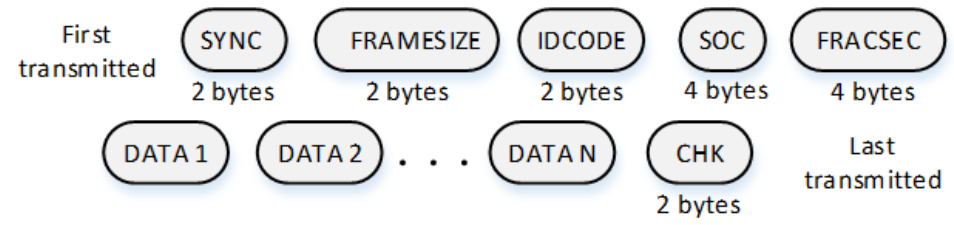

Figure 9.4 Common word definition

The PDC continuously sends a "command frame," requesting from available PMU stations to send the "configuration frame". If the PMU station sends the configuration frame in response, the PDC sends another command frame, requesting PMUs to start sending the "data frame". Upon receiving a start command, the PMU starts to send data frames according to the message update rate.

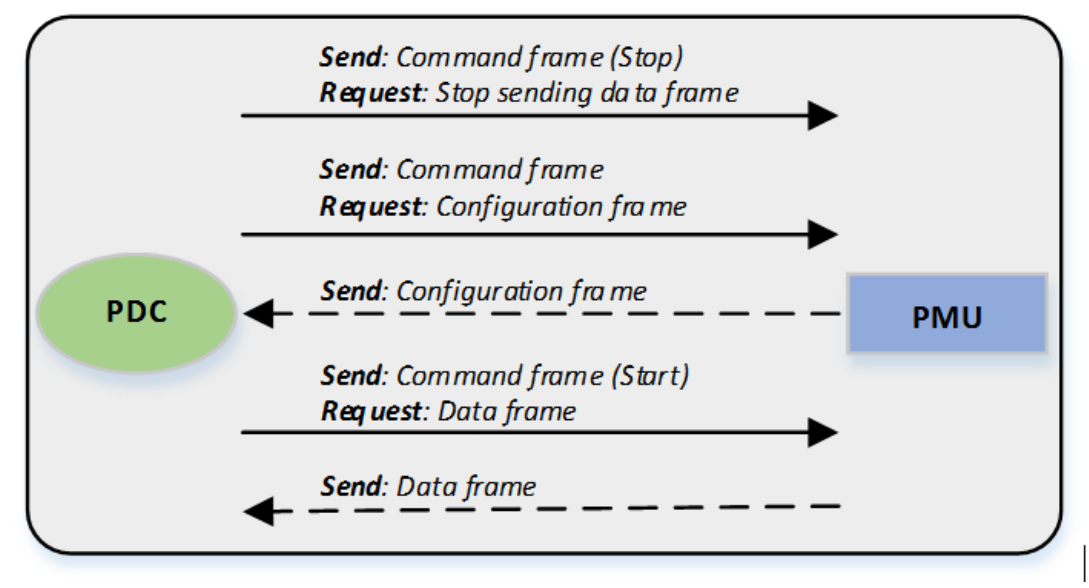

Figure 9.5 PDC and PMU data exchange

\subsection{Development of Laboratory-Based Phasor Measurement Unit}

As mentioned in Chapter 1, PMU has a well-known structure. In this section, we introduce the redefined structure of the laboratory-based PMU, according the available 
assets within the laboratory, and create our PMU by the following elements. In addition to the developed PMU, a commercial set of PMU and PDC from SEL is also installed in the ESRL testbed.

\subsubsection{IRIG-B Decoder}

The developed IRIG-B decoder is presented in Figure 9.6. When the GPS clock receives the UTC time information and exports it through IRIG-B, the IRIG-B decoder decodes the standard information and provides the UTC and the trigger pulse. An embedded circuit is developed to decrypt the TTL IRIG-B signal, to capture the time information, and to find the trigger instance. This provides us with the instantaneous time and the rising pulse for triggering the data acquisition process. The STM32F4 discovery board is used as the processing unit, along with a LCD to monitor the time and a XBEE module to transfer the UTC information.

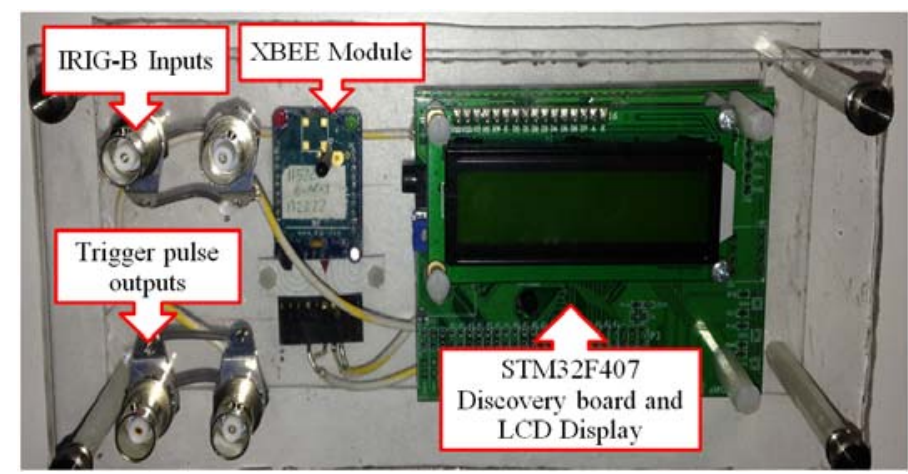

Figure 9.6 IRIG-B decoder prototype

A LCD is used to illustrate the real-time information. The XBEE module is used to publish the time information through a serial communication channel. The internal ARM processor has a $168 \mathrm{MHz}$ clock frequency and uses a digital input pin to receive the IRIG-B signal. A digital output pin is used to send the trigger instant with a UART 
channel to transfer the time data. The IRIG-B decoder algorithm is presented in Figure 9.7.

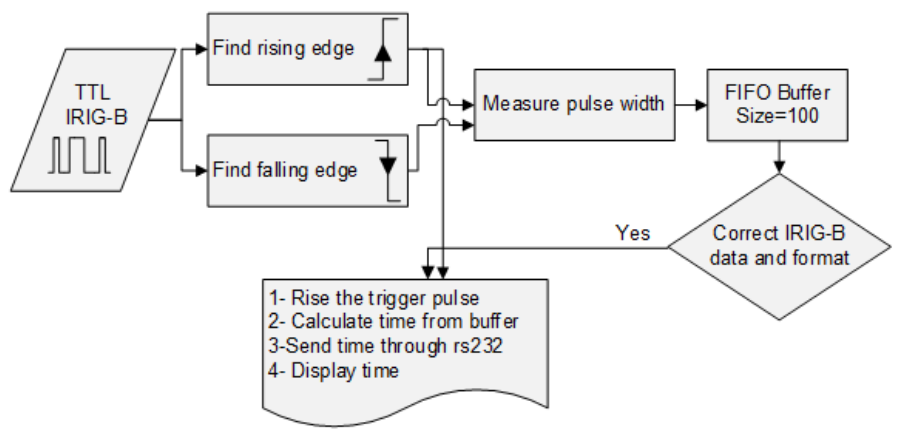

Figure 9.7 IRIG-B decoder algorithm

\subsubsection{Processing Unit}

The NI LabVIEW environment is used for processing the data due to its capabilities in communications, visualization, hardware interface, and strong and numerous libraries of calculation functions.

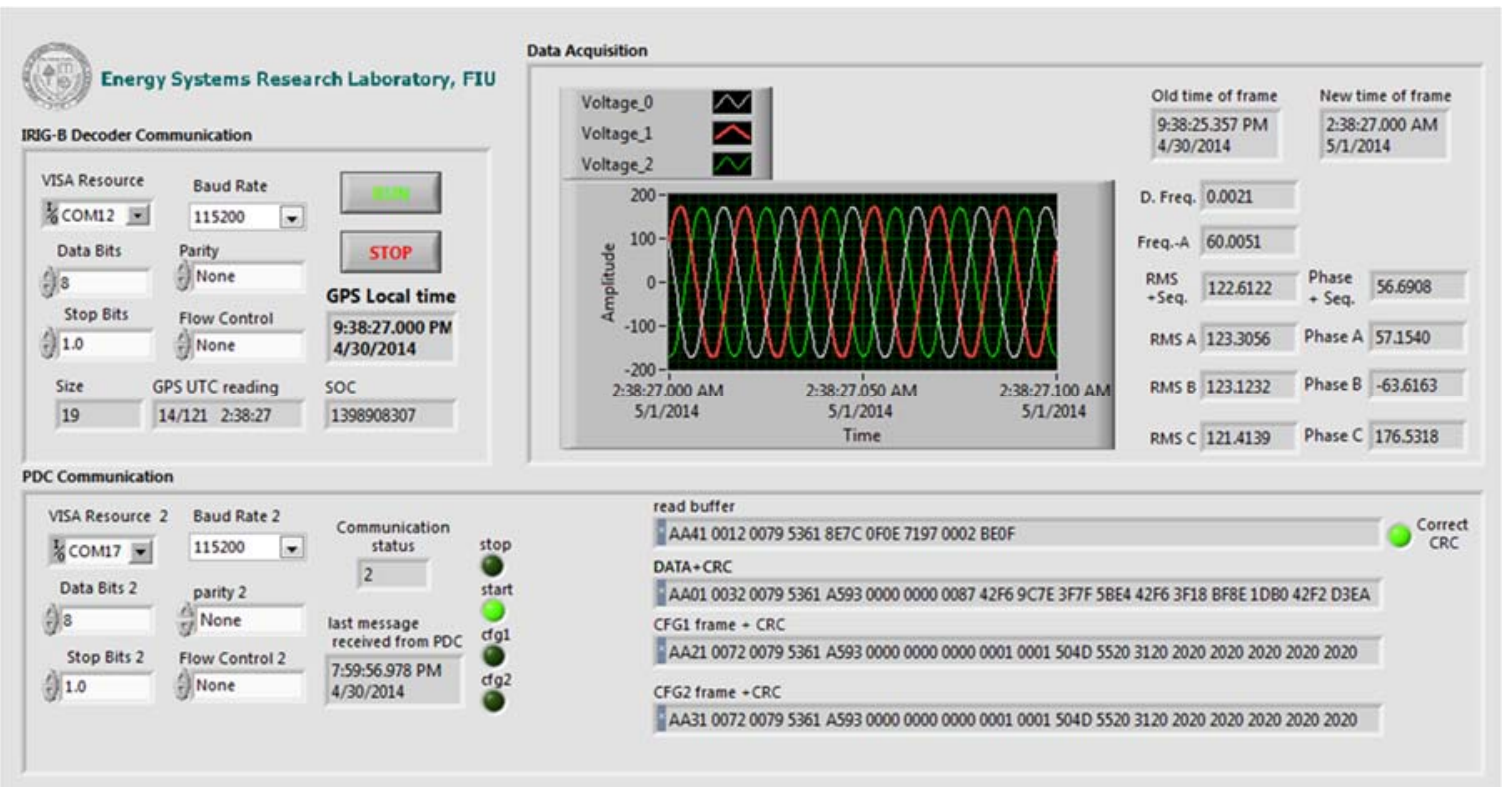

Figure 9.8 PMU front-panel 
Figure 9.8 shows the front panel of the developed PMU. A window-of-samples is processed, and the power system measurements are obtained.

\subsubsection{Data Acquisition}

The data acquisition module captures the real-time measurements from the waveforms of the power system network. The processing unit calculates the input signal, attaches the time-stamp, and establishes the communication terminal in order to transfer

the phasor measurements. The acquisition of captured windows-of-samples is triggered by the provided rising edge pulse from the IRIG-B interpreter. Depending on the application, the sampling frequency might be different.

A National Instrument DAQ9206 device, which has 32 analog input channels, is used with a RSE/NRSE (referenced/non-referenced single-ended measurements) connection to capture the voltage signals. The maximum sampling rate of the device is $250 \mathrm{kS} / \mathrm{s}$, which corresponds to a $4 \mu \mathrm{s}$ delay per channel. This error for a $60 \mathrm{~Hz}$ sinusoidal waveform can cause about 0.0864 degree cumulative phase shift per channel. The resulting error needs to be compensated when multi-channels are employed. For instance, when the frequency is $59 \mathrm{~Hz}$, the phase error compensation for the $3^{\text {rd }}$ channel results in:

$$
59 * 360 * 0.000004 *(3-1)=0.08496 * 2 \text { (degrees) }
$$

\subsubsection{Calculation and Communication}

During the calculation period, the time information is received from the IRIG-B decoder, through the serial port, which does not require high-speed communication. The reported measurements should be in a synchrophasor standard format, so that they can be accessed from the PDC. Figure 9.9 demonstrates the structure of the developed PMU. 


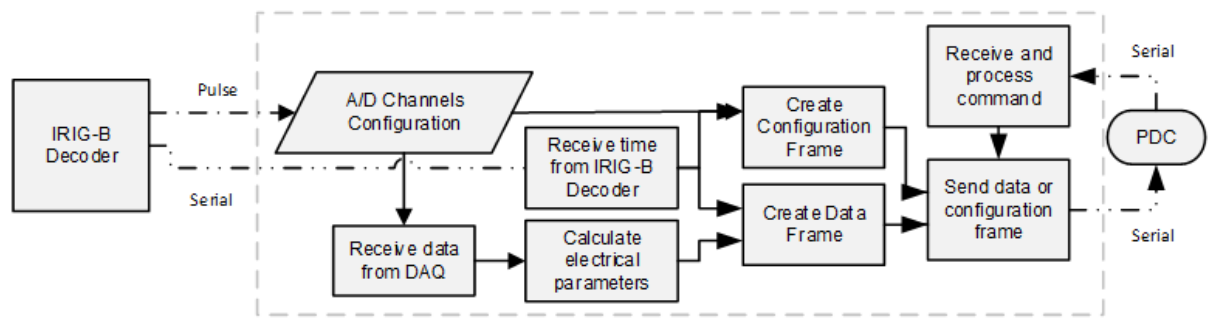

Figure 9.9 The software structure of the developed PMU

The experimental hardware test platform in Energy Systems Research Laboratory (ESRL) at Florida International University is entirely described in Chapter 2 and Chapter 3 of this dissertation. This platform was used to test and verify the results of the developed PMU.

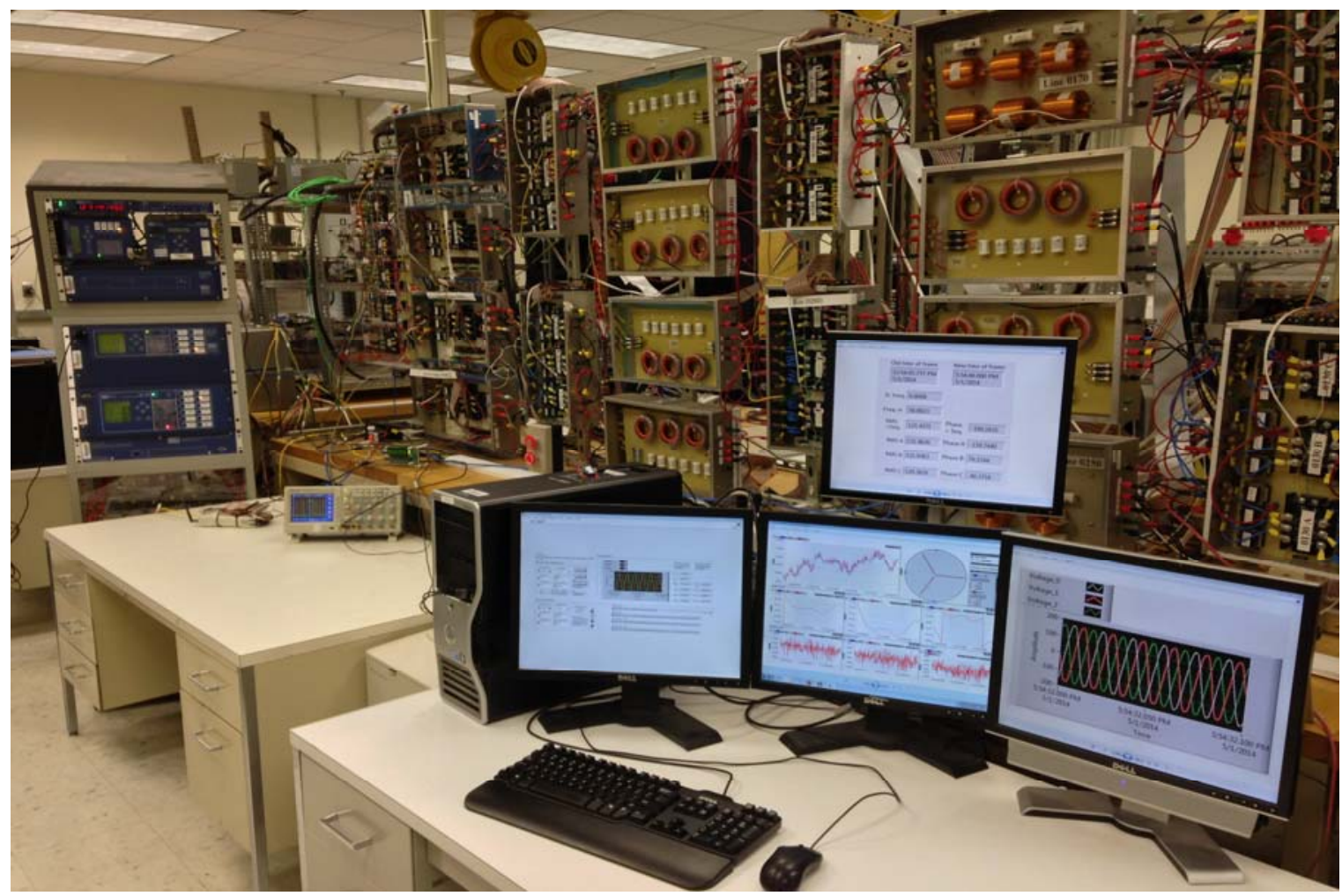

Figure 9.10 Experimental setup

Figure 9.10 shows the hardware setup and the testing platform for the developed PMU. Voltage transformers are used to scale down the measured voltage from the 
nominal 120 Vrms to 6 Vrms in order to be measured through DAQ analog inputs, which have a limited input range of $+/-10$ Volts. Current transformers are used to convert the actual current into a voltage form signal, and operate in a linear range (1 Volt corresponds to 3 Amps).

\subsubsection{Off-the-Shelf PMU Implementations}

Many vendors around the world are manufacturing PMU units with a high quality and high message reporting rates, according to synchrophasor standards[128], [129]. This testbed owns two commercial PMUs integrated with a PDC and a real-time automation controller setup.

The PMU enables us to capture time-synchronized measurements, such as frequency, phase angle, and amplitude of the voltage and current phasors. Moreover, the obtained measurements can be utilized to provide a logical feedback to the power system field equipment, such as Real-Time Automation Controllers (RTAC), Programmable Logic Controller (PLC), and the embedded controllers. The RTAC supports many industrial protocols, such as synchrophasor protocol (C37.118), fast messaging protocol (SEL), and MODBUS. A PLC is used by the research team to control the generator governor emulator through the frequency driver and circuit breakers of the load models. An embedded controller is used to control an inverter of the Flexible AC Transmission System (FACTS). A commercial PMU setup available at the ESRL testbed is illustrated in Figure 9.11. 


\subsection{Validation, Calibration, and Performance Comparison}

In order to evaluate the developed PMU, 3 experiments have been performed: first, to evaluate the performance of the IRIG-B time decoder, second, to perform Total Vector Error analysis, and finally, to evaluate the true phase and frequency for the developed PMU.

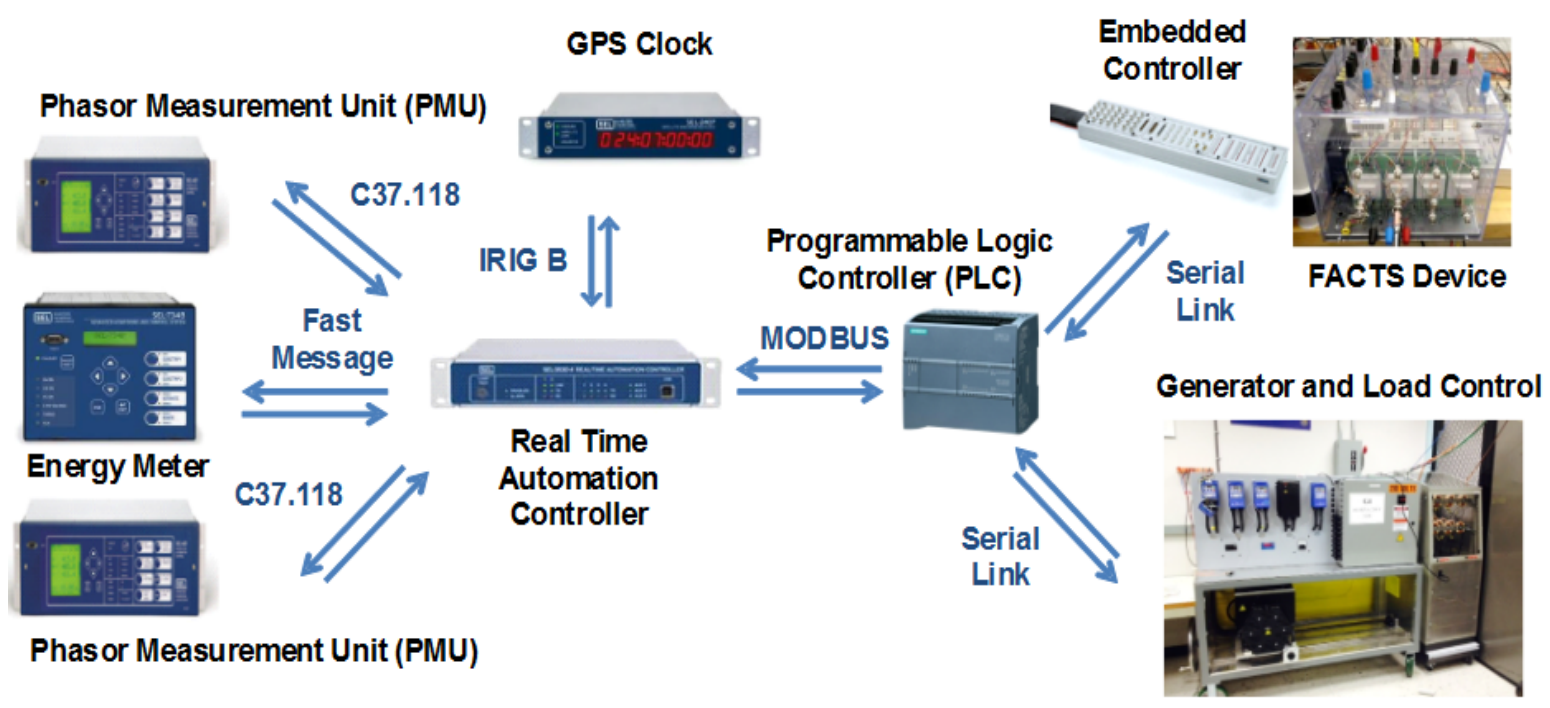

Figure 9.11 Integrated PMU and PDC setup with PLC and RTAC

\subsubsection{Evaluation of the IRIG-B Decoder}

The IRIG-B decoder is responsible for providing a pulse at the instance of the time change in the IRIG-B format. The embedded decoder has a delay in responding to the triggering event. Figure 9.12 shows the measured delay between the instance of the time change and the instance when the pulse is applied to the output port of the IRIG-B decoder device. As it has already been defined in Section 6.2.3, the maximum time delay is less than 1.6 micro-seconds, which is $7 \%$ of the maximum acceptable range (26 us). 


\subsubsection{TVE Comparison between the Developed PMU and Commercial PMUs}

One of the criteria to evaluate the accuracy of PMUs is the Total Vector Error (TVE) and it is defined in [129].

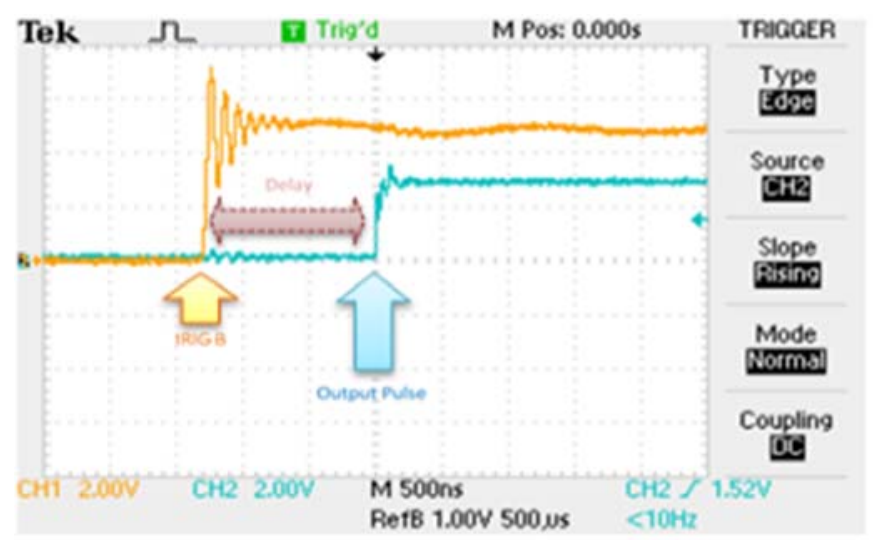

Figure 9.12 Measurement of the total delay in IRIG-B decoder module

As mentioned before, TVE expresses the magnitude, phase, and timing error and it is based on the standards, it has to be less than $1 \%$.

Figure 9.13 shows the results for the developed PMU, and it is within the acceptable margin of the TVE standard. Figure 9.13 also illustrates the TVE for phase A of the developed PMU with respect to actual commercial PMU readings for a one-minute experiment with the rate of 1 message per second. The captured window was selected randomly, and the values are measured based on Equation (9.6).

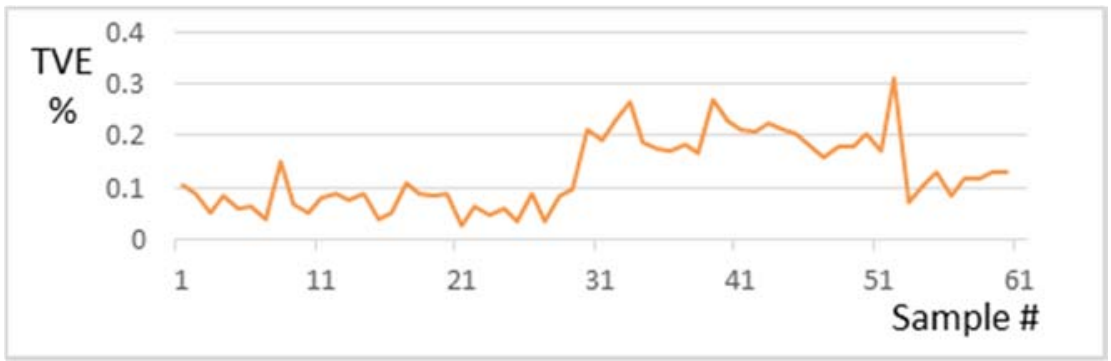

Figure 9.13 Total Vector Error 
The abovementioned experiment was executed to validate the TVE for the laboratory-based PMU with respect to commercial PMUs.

Figure 9.14 shows the final measurements available in the PDC software. They are collected from the developed PMU (PMU 1) and the commercial PMU (PMU421). The result is depicted on a web-based monitoring software, which is polling the information from the PDC. In this experiment, we assume that the SEL measurements are $100 \%$ true; as seen from the results, the developed PMU successfully tracks the assumed reliable measurements. This figure expresses the followings:

- The graphs on the top row show the frequency and three-phase angle measurements.

- The graphs in the middle row show the phase angle value of each phase.

- The phase angle differences between the developed and commercial PMUs are shown on the graphs in the bottom part row.

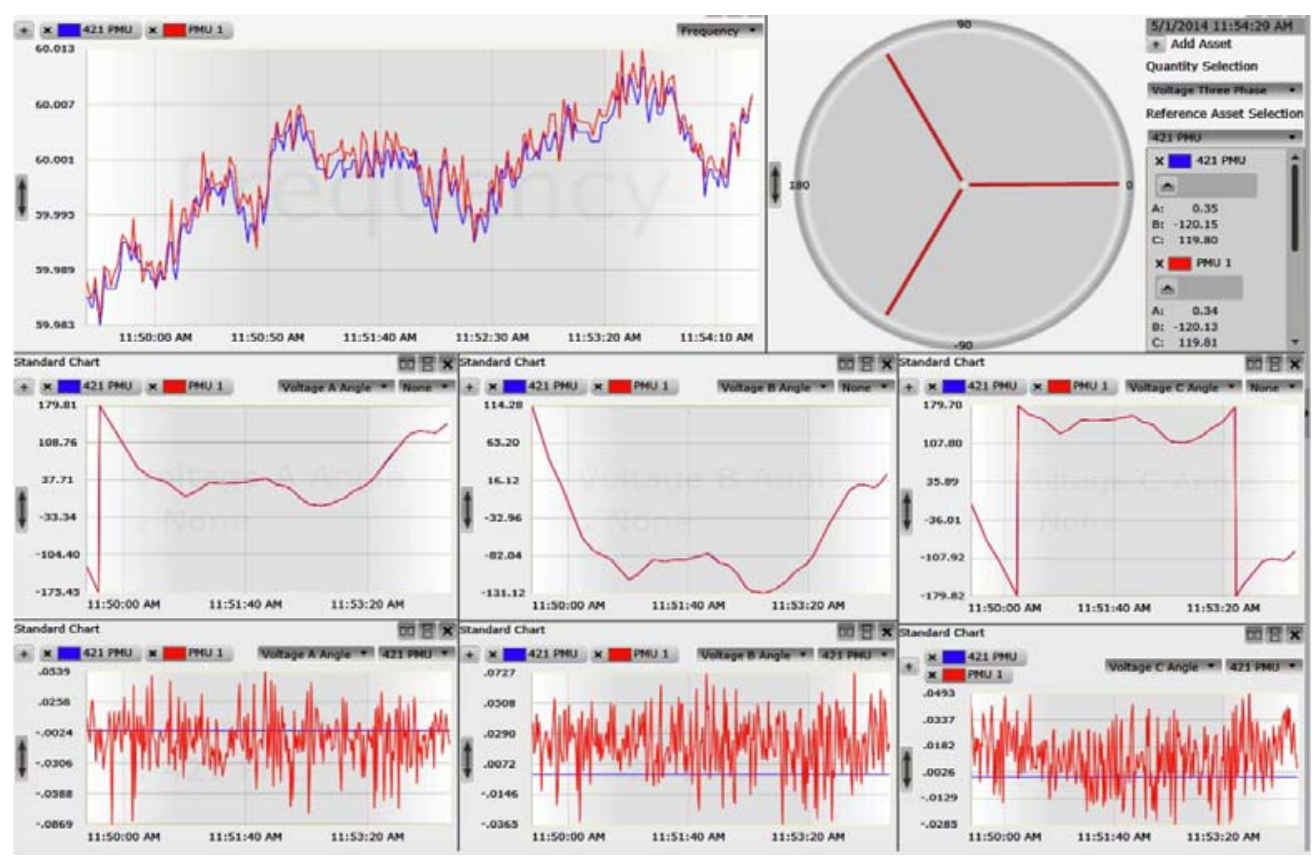

Figure 9.14 Measurements available in PDC 


\subsubsection{PMU Calibration}

As mentioned in Section 6.1, using multi-channel unsynchronized DAQs will result in deviation of phase measurements due to the delay, which came from the multiplexing technique. Figure 9.15 shows the results, which have the abovementioned delay and have not been calibrated yet. In this figure, the PMU421 is assumed as the correct measurement; the results of the developed PMU are compared based on the PMU421 as the true reference.

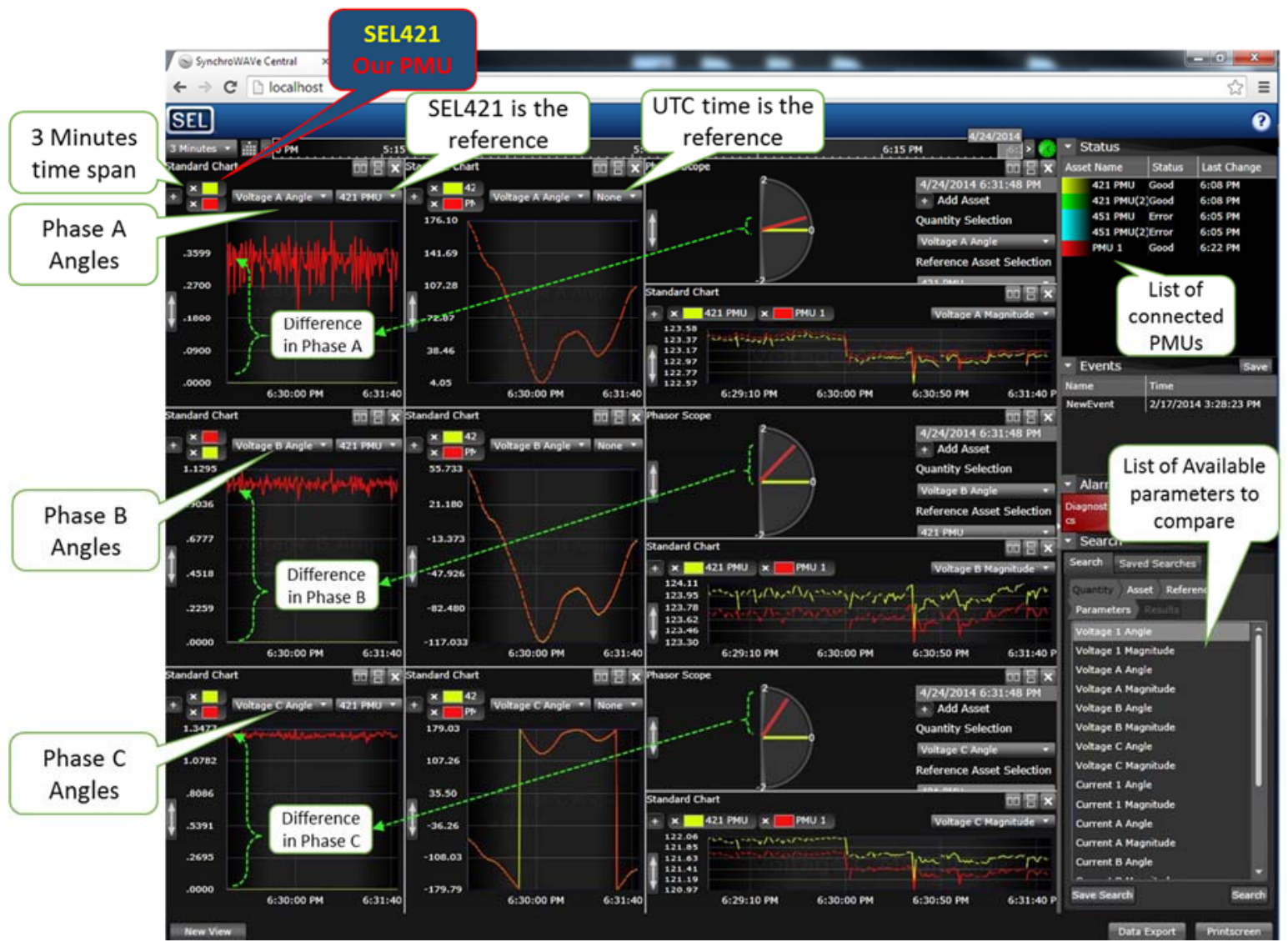

Figure 9.15 Comparison of phase angle measurements from SEL421 PMU and developed PMU without offset calibration 
After calibrating the PMU according to Equation (6.7), the results will be in an acceptable range. Figure 9.16 shows the results after the calibration and based on the PMU421 as the reference.

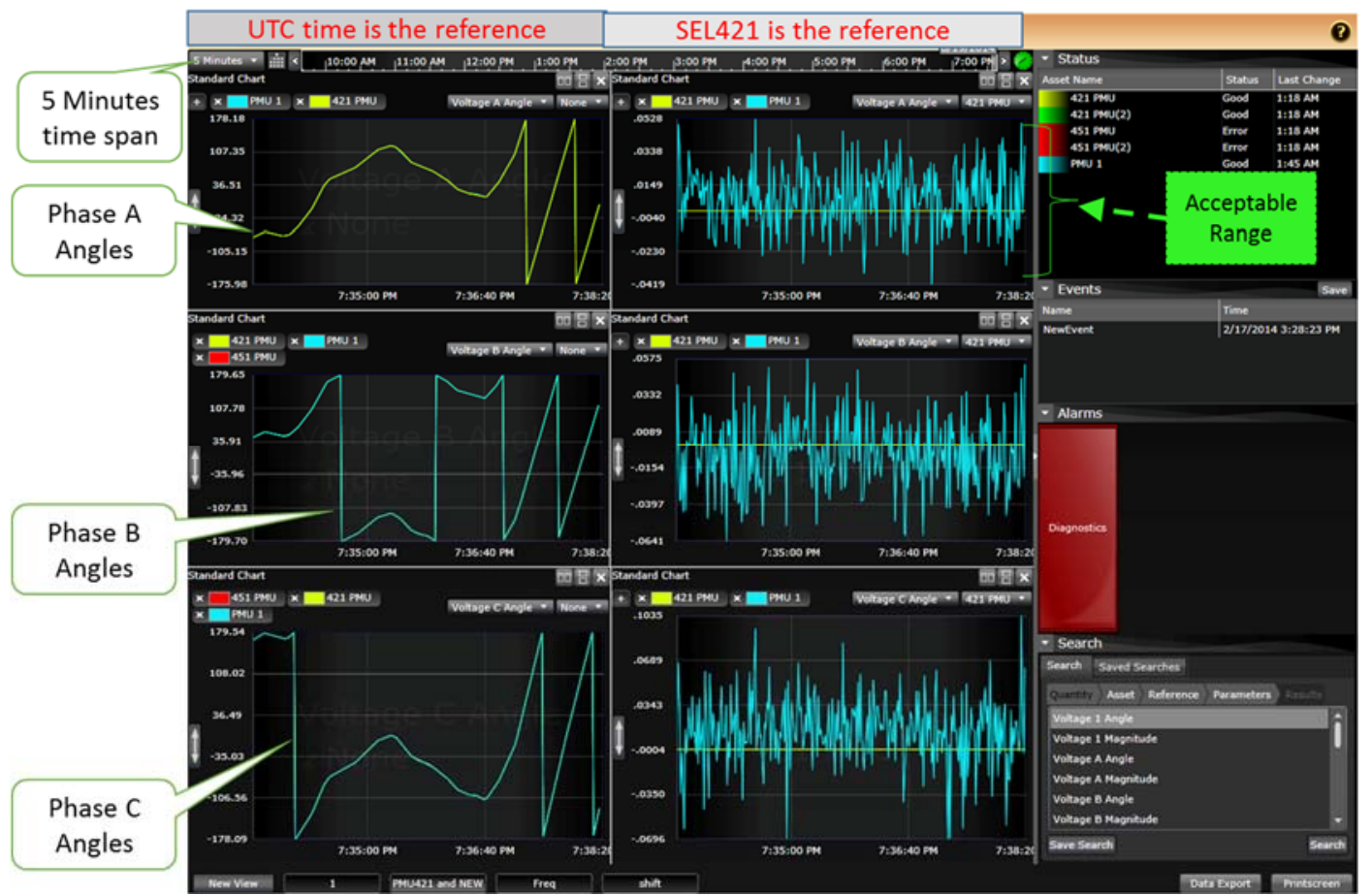

Figure 9.16 Comparison of phase angle measurements from SEL421 and developed PMU with offset calibration 


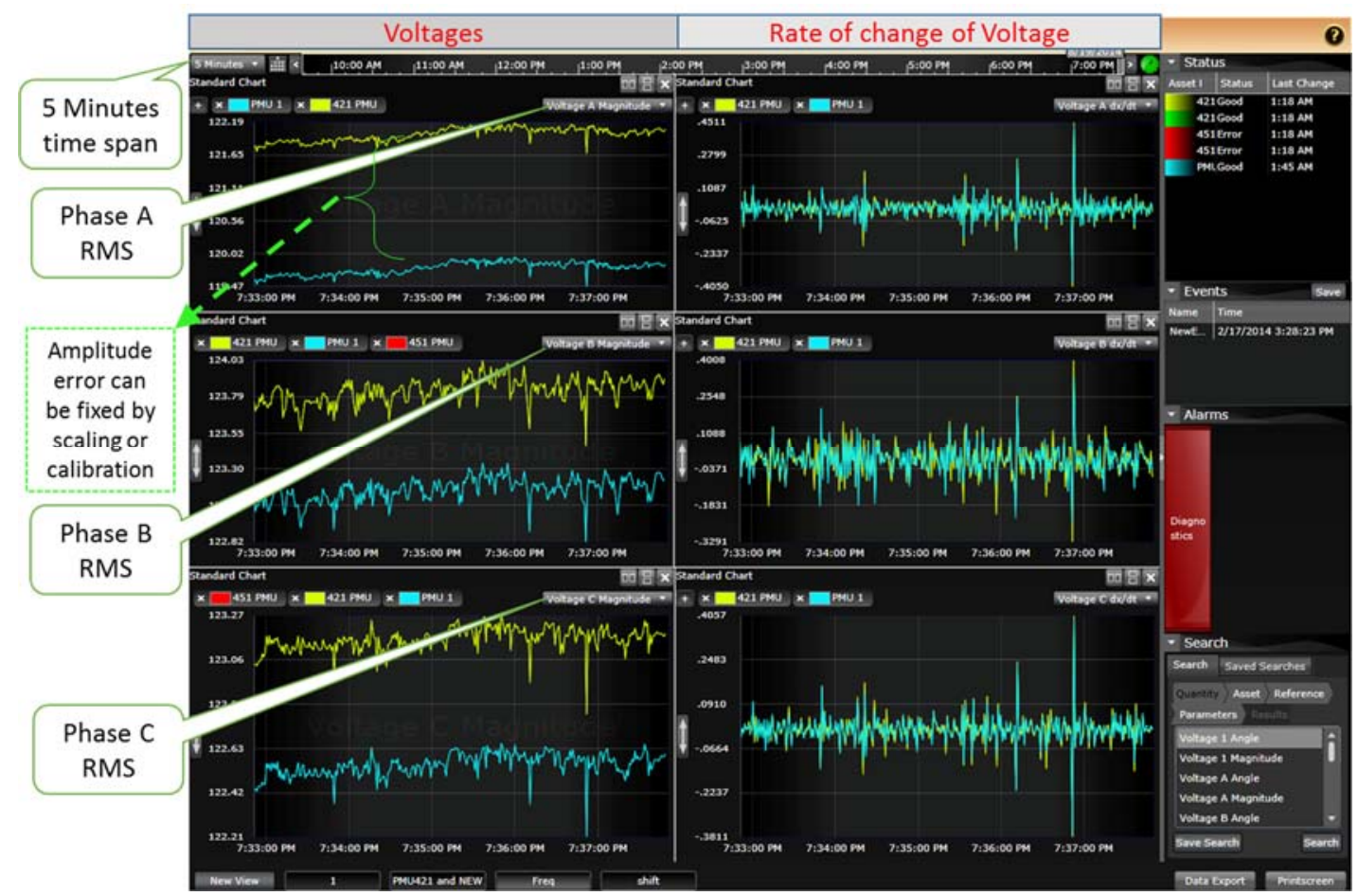

Figure 9.17 Comparison of voltage and rate of change of voltage from SEL421 and Developed PMU

For voltage/current amplitude measurements, the calibration can be performed through scaling potentiometers. The results are within an acceptable range and an example of measurements is presented in Figure 9.17.

Figure 9.18 shows the phasor diagram representation of the measured phases for the developed PMU and based on PMU421 as accurate references. It also includes the measured frequency. The results indicate that the maximum deviation is less than 0.02 $\mathrm{Hz}$, which certifies the accuracy and the precision of the developed PMU. 


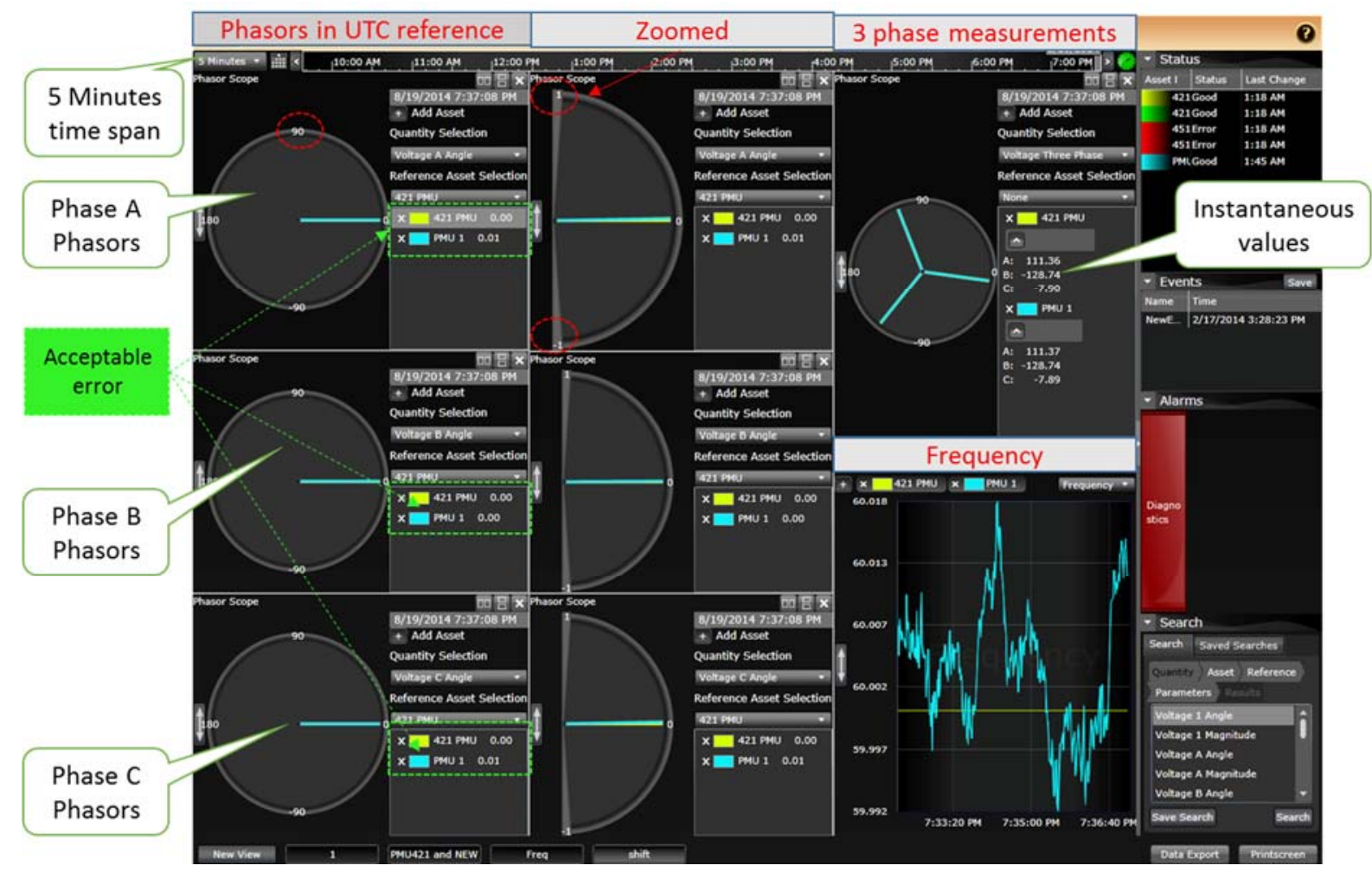

Figure 9.18 Comparison of phasors and frequency measurements from SEL421 PMU and developed PMU

\subsubsection{Evaluation of the captured Phase and Frequency}

In order to make sure that the developed PMU is accurate enough, and also to find its error in the process of measuring a time-stamped frequency and phase, the phase and the frequency have been manually measured using a high-precision oscilloscope.

Figure 9.19 shows a captured waveform of Phase A along with the IRIG-B signal, as well as the trigger signal for DAQs.

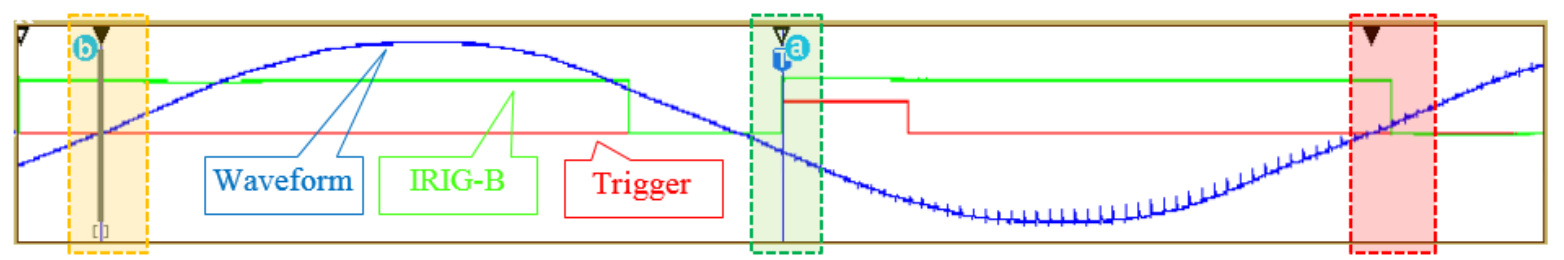

Figure 9.19 Captured waveforms by high resolution oscilloscope 
Figure 9.20 represents the expanded time scale of the highlighted green area in Figure 9.19. It also confirms the results from Figure 9.12 regarding the IRIG-B decoder delay analysis.

This timing error has been improved in upgraded design of IRIG-B decoder to less than 200ns.

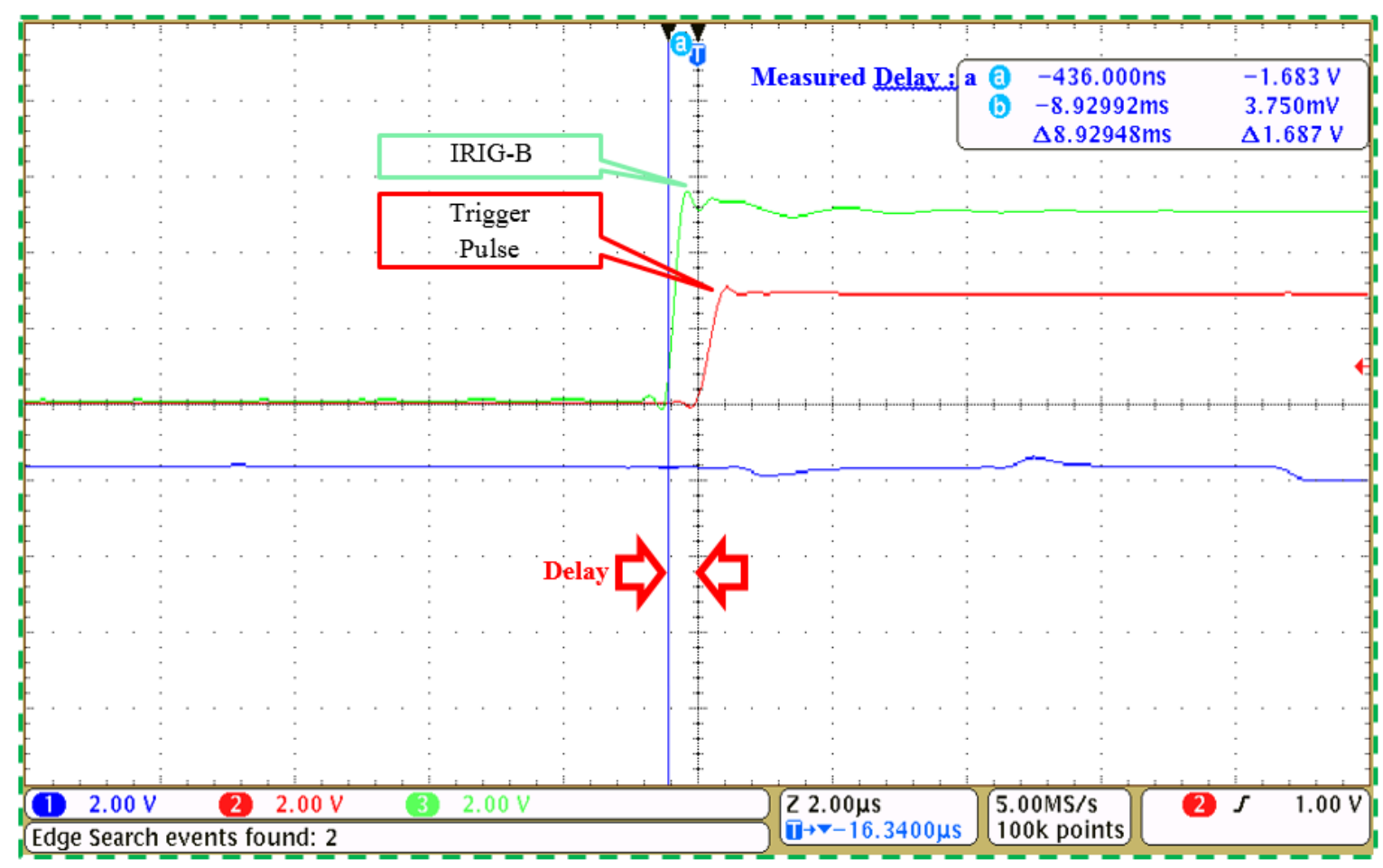

Figure 9.20 Enlarged area for green box in Figure 9.19 


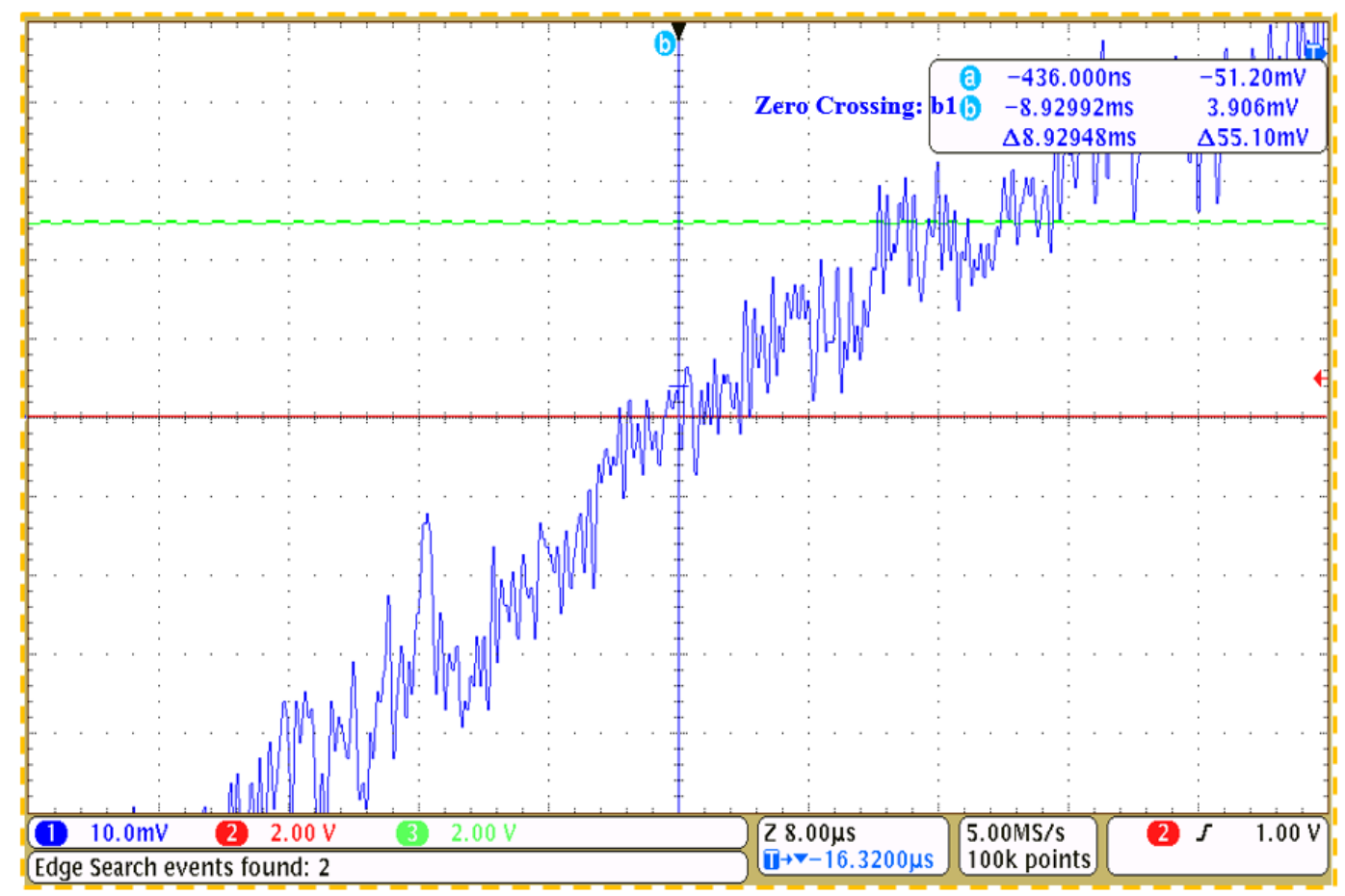

Figure 9.21 Enlarged area of yellow box in Figure 9.19

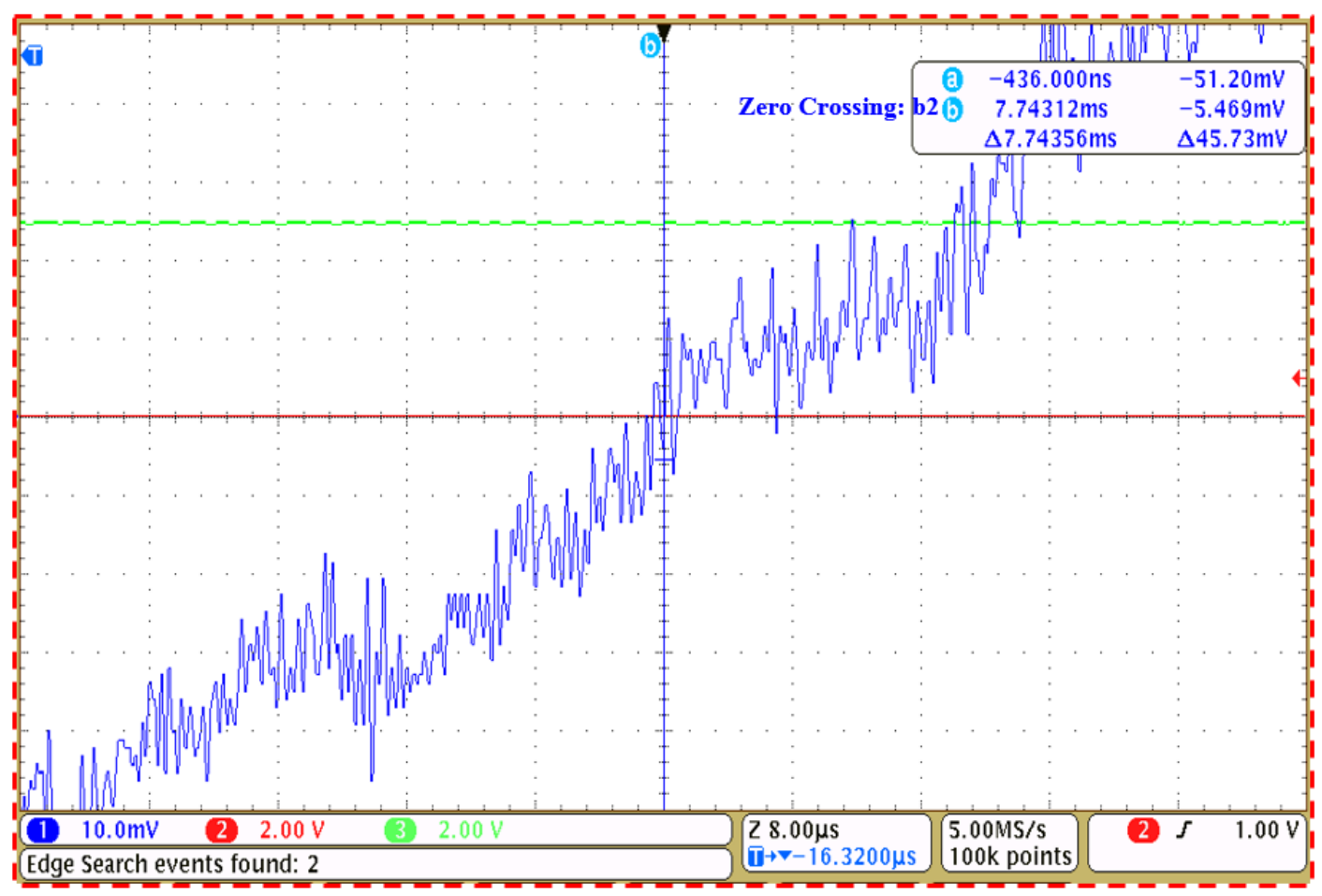

Figure 9.22 Enlarged area of red box in Figure 9.19 
Available measurements from Figure 9.20, Figure 9.21 and Figure 9.22 provide the time reference, zero crossings of the same cycle, and the delay of the trigger signal, respectively. The frequency of the waveform can be found using zero crossing measurements and through the waveform period $(\mathrm{T})$.

$$
F=\frac{1}{T}=\frac{1}{b 2-b 1}
$$

In addition, the phase of time instant with respect to zero crossing can be found through:

$$
P h=\Delta t * 360 * F=(a-b 1) * 360 * F
$$

In the above equations, ' $a$ ' is the IRIG-B instant, ' $b l$ ' is the zero crossing at beginning of the cycle, and ' $b 2$ ' is the zero crossing at the end of the same cycle, and they are clearly presented in Figure 9.20,Figure 9.21 and Figure 9.22.

In order to check the performance, several random measurements with different timings have been performed and their results have been reported in Table 9.1.

\begin{tabular}{|c|c|c|c|c|c|c|c|c|c|c|}
\hline \multicolumn{2}{|c|}{ 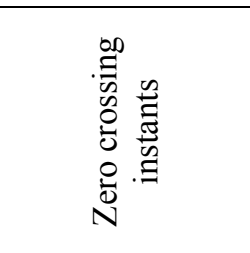 } & \multirow{2}{*}{ 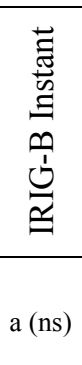 } & \multirow{2}{*}{ 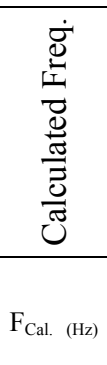 } & \multirow{2}{*}{ 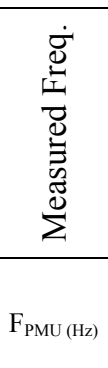 } & \multicolumn{2}{|c|}{$\begin{array}{l}\dot{D}=0 \\
\dot{0} \\
\dot{D} \\
\dot{d}\end{array}$} & \multirow{2}{*}{ 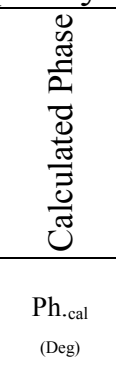 } & \multirow{2}{*}{ 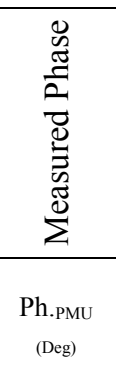 } & \multicolumn{2}{|c|}{ 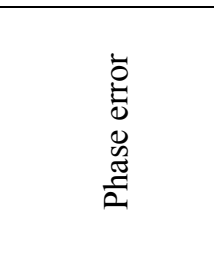 } \\
\hline b1 (ms) & b2 (ms) & & & & $(\mathrm{Hz})$ & $\%$ & & & (Deg) & $\%$ \\
\hline-7.7476 & 8.92128 & -500 & 59.9920 & 59.9993 & -0.0073 & 0.0073 & 77.3371 & 77.2852 & 0.0526 & 0.0671 \\
\hline-12.0278 & 4.65527 & -1200 & 59.9409 & 60.0054 & -0.0645 & 0.1076 & 169.5186 & 169.3314 & 0.1872 & 0.1104 \\
\hline-8.92992 & 7.74312 & -436 & 59.9770 & 59.9850 & -0.008 & 0.0133 & 102.8029 & 102.7100 & 0.0929 & 0.0903 \\
\hline-3.9830 & 12.66696 & -848 & 60.0602 & 59.9857 & 0.0745 & 0.1240 & 176.1007 & 176.1179 & -0.0172 & 0.0097 \\
\hline
\end{tabular}

Table 9.1 Random results for phase and frequency measurements 


\subsection{Experimental Application of PMUs}

For power system experts, who deal with complex wide-area problems, learning the PMU structure is not enough by itself. In this section, first we developed several experiments to take advantage of the implemented PMU results. Then, we will utilize PMUs to extract power system parameters to evaluate online transmission line parameter identification. This is achieved due to the fact that PMUs can provide the synchrophasor information. Traditional approaches are not capable of sending control, protection command or monitoring alarms. Recent solutions have combined the aforementioned features in a multifunction device called the Phasor Measurement and Control Unit (PMCU).

\subsubsection{Transmission Line Parameter Identification}

In order to find the hot line real-time electrical parameters of the transmission line between two buses, PMUs can be deployed as shown in Figure 9.23. The pi-model of the transmission line is utilized for this representation. Accordingly, Figure 9.23 also shows the corresponding electrical parameters in each transmission line. The relationship between phasors and line parameters can be obtained, as in the following equation:

$$
\left[\begin{array}{c}
V_{s} \measuredangle \delta_{s} \\
I_{S} \measuredangle \theta_{s}
\end{array}\right]=\left[\begin{array}{cc}
1+\frac{Y Z}{2} & Z \\
Y\left(1+\frac{Y Z}{4}\right) & 1+\frac{Y Z}{2}
\end{array}\right] \times\left[\begin{array}{c}
V_{r} \measuredangle \delta_{r} \\
I_{r} \measuredangle \theta_{r}
\end{array}\right]
$$

In which $\vec{Z}$ is the line impedance $(\vec{Z}=R+j X), \vec{Y}$ is the line admittance $(\vec{Y}=$ $G+j B)$, where both can be found by:

$$
Z=\frac{\left(V_{s} \measuredangle \delta_{s}\right)^{2}-\left(V_{r} \measuredangle \delta_{r}\right)^{2}}{\left(V_{r} \measuredangle \delta_{r}\right)\left(I_{s} \measuredangle \theta_{s}\right)+\left(V_{s} \measuredangle \delta_{s}\right)\left(I_{r} \measuredangle \theta_{r}\right)}
$$




$$
Y=2 \frac{\left(I_{s} \measuredangle \theta_{s}\right)-\left(I_{r} \measuredangle \theta_{r}\right)}{\left(V_{s} \measuredangle \delta_{s}\right)+\left(V_{r} \measuredangle \delta_{r}\right)}
$$

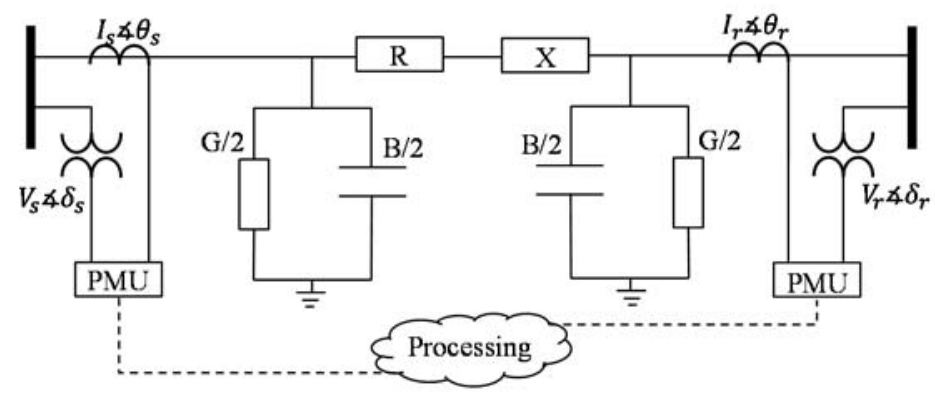

Figure 9.23 Pi-model of transmission line

Figure 9.24 illustrates the experimental results for the online calculation of the transmission line impedances, in which the phase $\mathrm{C}$ measurements of the sending and receiving ends are utilized. PMU451 is located at the sending end and PMU 421 is located at the receiving end of the transmission line.

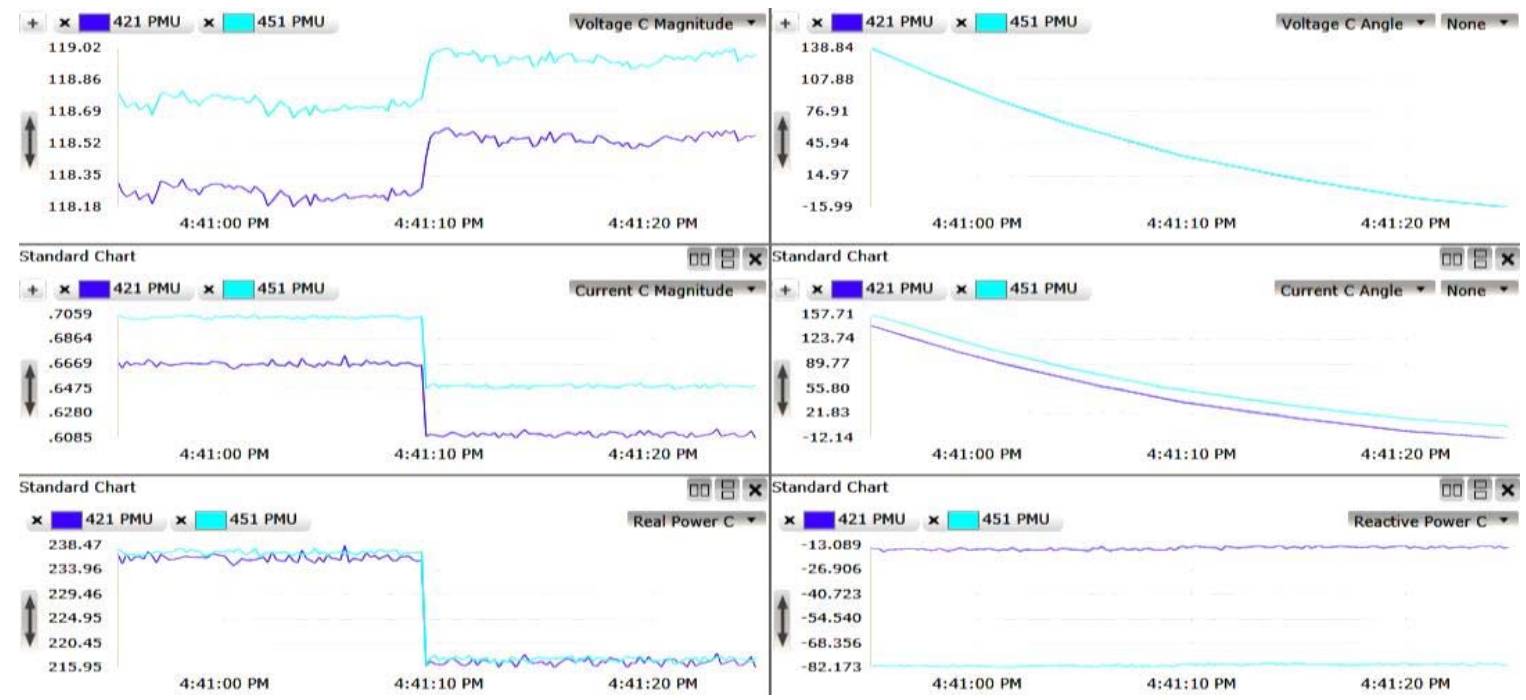

Figure 9.24 a) Voltage magnitudes b)Voltage angles c)Current magnitudes

d)Current angles e) Active power f) Reactive power 
The experiment period is 30 seconds, with the rate of 5 synchrophasor messages per second. Initially, a resistive load, which draws approximately 235 Watts, was utilized. The load value was switched to 215 Watts at "4:41:09" in order to investigate the impedance variation according to the loading conditions.

Online calculation of the Resistance $(R)$, Reactance $(X)$, Conductance $(G)$ and the Susceptance $(C)$ are shown in Figure 9.25 and Figure 9.26.

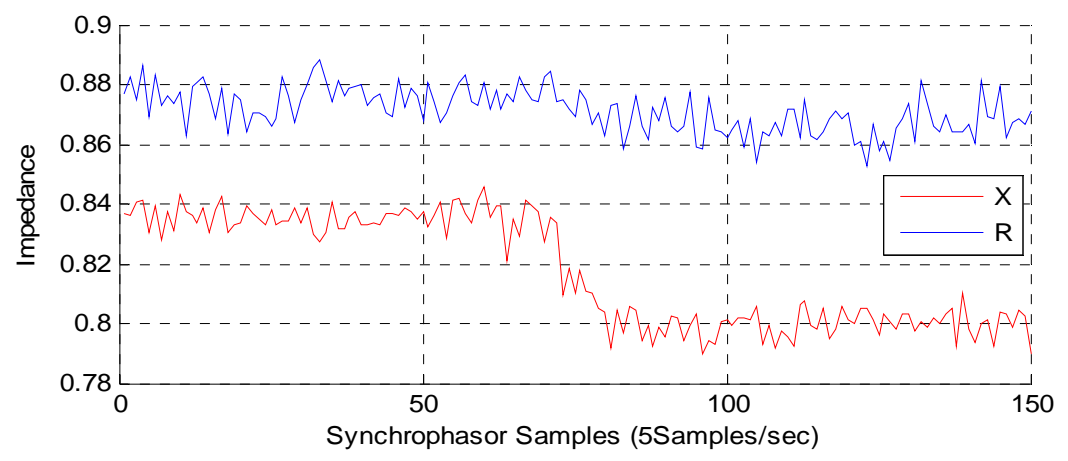

Figure 9.25 Calculated resistance and reactance

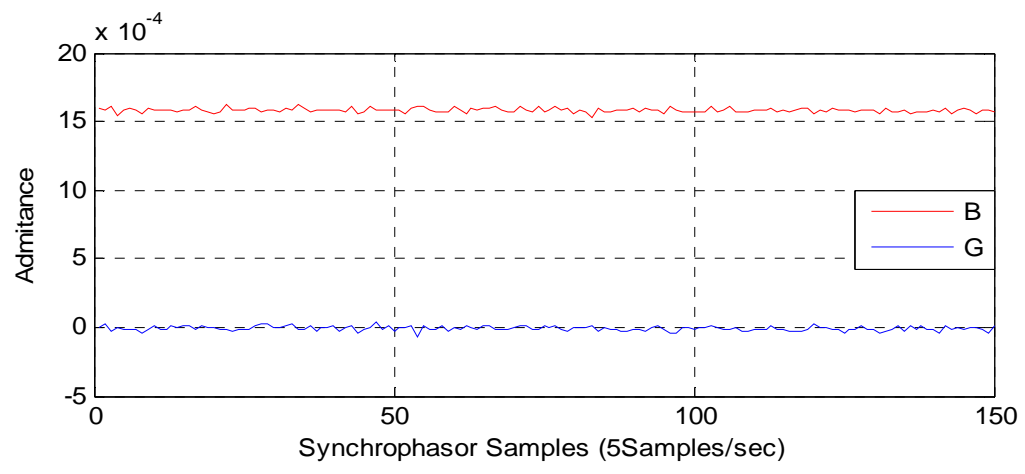

Figure 9.26 The Calculated susceptance and conductance

\subsection{Conclusion}

Integration and application of Phasor Measurements Units are going to be one the main orientations of future power systems. Investigation in the communication, security, precision improvement, and application of PMUs would be promising when they are 
involving actual physical components and under complete authority of such devices. In this chapter, in addition to describe the main aspects of PMU structure, a laboratorybased open source phasor PMU was developed. The precision and performance of prototyped device was compared with a commercial sample; moreover, its error in timing, phase and frequency measurements were calculated in time domain and by using a high precision oscilloscope. Finally, a real-time technique for measuring the parameters of power transmission lines by utilizing PMU technology was introduced and employed on an experimental setup. 


\section{DEVELOPMENT OF TECHNIQUES FOR ISLANDING DETECTION USING PHASOR MEASUREMENT UNITS}

This chapter presents the issues involved in the detection of islanding via traditional methods, as well as the implementation of 3-phase synchronized measurements. A proposed technique for better islanding recognition in the microgrids using multiple PMUs was developed. A new method which uses 3-phase synchronized measurements is introduced for the detection of unbalanced or partial islanding situation of the microgrids. The partial islanding occurs, when the microgrid loses only one phase of its link to the main grid. In addition to simulation of the proposed method in MATLAB/Simulink, experimental results are also obtained. A small-scaled 3-phase power system at the laboratory scale was developed and used to compare the traditional and new techniques. The comparison of experimental and simulation results shows successful operation of the proposed technique and verifies the results consequently.

\subsection{Introduction}

Islanding is the situation when a portion of the power system, which includes both generations and loads, is isolated from the main grid, but stays energized. It may lead to hazardous situations within the island. Disadvantages of islanding situation are mainly safety hazards for utility workers and customers, and equipment's damage due to the uncontrolled properties of frequency and voltage. Fast and reliable islanding detection methods can avoid potential risks, such as frequent maintenance, poor power quality, and reclosing problems. The detection should be followed by control decisions to maintain 
the system stability and performance, and it should match the load to available generation, while it is maintaining the power quality. It was recommended to detect and cease to energize the area within 2 seconds of the formation of an island [130].

Until now, many standards have been released and many detection techniques for islanding have been proposed and tested. They are mainly divided into two types. Remote techniques, which mostly use SCADA system and power line communication method. The second type depends on using the local techniques, classified into two major sets. In passive methods, the local electrical parameters are measured and in case of any deviation from preset value, islanding indices can detect the isolation from the main grid. This method is usually using over and under frequency protection relays, active and reactive power measurement, voltage, and also rate of changes of frequency (ROCOF).

In the active methods, one is the injection of reactive power at a level in the connection point of generator to utility. This reactive power flow can only be maintained as long as generator and utility are connected continuously. By using the Reactive Power Export Error Detection relay (RPEED) islanding will be recognized through the lack of reactive power flow. Depending on types of the excitation system or the generator, this method could be useless.

With recent developments in faster communication and using synchronized measurements and Phasor Measurement Units, performance of smart grids have been enhanced. PMUs found numerous applications in identification and evaluations of power system states. One of them is the islanding detection. In fact, they measure multiple parameters synchronized by a time reference, so they can be used for islanding detection too; but using only positive sequence information may deviate the recognition process in 
many abnormal conditions. Islanding detection techniques should be robust and reliable. An evaluation of detection methods should consider the following parameters[53], [131]:

- Reliability of islanding detection

- $\quad$ Time requirements of IEEE STD 1547

- Impact on the grid and power quality

- Cost

- Flexibility to reconfiguration of grid

They should also be capable of working under challenging and abnormal conditions of microgrids such as:

- Short circuit faults

- Unbalanced isolation of microgrids

- $\quad$ Reactive load switching

- $\quad$ Sudden load change

- $\quad$ Sudden load power factor change

Mentioned methods have problems [132] and for this reason an improved method was proposed in this chapter to form an islanding detection method with new capabilities.

At first, we will discuss a brief overview of islanding detections methods and drawbacks of each method. It is followed by the new proposed method and the algorithm of islanding detection using phasor measurement units. Simulation of proposed method is carried out in Simulink environment. Finally, by explanation of hardware test setup and the islanding scenarios followed by experiments results, comparisons are made and the conclusion is justified. 


\subsection{Islanding Detection Methods}

Islanding detection techniques are mainly categorized in 2 groups of remote and local methods. Remote methods, such as SCADA systems, are based on collecting information from a field to convey other regional sites or to a central station. Since the primary concern is to monitor and send an alarm from a central station, SCADA systems may not respond accurately and timely to islanding detection and react to the corresponding generator due to communication delays. For this case, local islanding detection is more effective, compared to remote decision. Moreover, implementing a SCADA system requires higher investment, compared to effective local detection technique. Local detections are mostly based on small relays, which are coupled to generation units[133], [134]. Another application of SCADA is to monitor the status of breakers and find the islanded area through the current scheme of the grid, which is called the transfer trip scheme. This method requires huge communication support. Recently, internet-based methods provide better communication infrastructure, but they can barely be recommended as the primary method of protection in islanding detection [135].

Power Line Carrier Communication (PLCC) is another remote method which continuously sends a low energy communication signal along the power line and through a transmitter connected to grid. The receiver is located in the other side and will detect the islanding situation based on receiving the PLCC signal. This method is also not applicable due to expensive costs in small DG systems [136].

Local methods use the information at the DG side and they are divided into passive and active methods. Passive methods rely on local measurements to find sudden changes in electrical parameters. Active methods use power system operation and apply 
small perturbations for significant changes in system parameters when DG is islanded[137], [138].

Rate of Change of Frequency (ROCOF) relays are the most common application in the existing distribution network. This passive technique uses power imbalance to detect frequency change in the system, where the speed of change of frequency is related to power imbalance amount between the network and the islanded system. The mismatch between load and generation causes a sudden change of frequency as a function of mismatch rate and inertia of the generator.

Vector surge relays are another passive method, which operates by monitoring the phase angle difference between terminal voltage and generator's internal voltage. The phase difference between two consecutive zero-crossing points varies rapidly in the case of grid failure. If phase angle exceeds the defined threshold value, a generator relay will trip [139].

Over /under voltage/frequency and the techniques that rely on harmonic distortion are other examples of the passive method[140]. Inserting a small impedance, usually by a capacitor bank at the utility side of PCC, will be another solution to finding the islanded area, through the change of reactive power balance. However, this method has its drawbacks, which mostly are the cost of the capacitor bank and low time response due to the switching time of the capacitor[53].

\subsection{New Method for Islanding Detection}

Modern relays, especially those with PMU potential, are capable of providing several types of information at the point of common connection (PCC). Requirements of 
the IEC 61850 standard enforce them to have fast and reliable communication in order to transfer their information within standard constraints. The frequency reference or the phase reference are the most used types of information. They have been used to identify the disconnection between the main grid and the microgrid [11], [49], [53], [54], [135].

\subsubsection{Unbalanced Islanding Detection}

According to C37.118, all PMUs must be able to measure 3-phase voltages and currents, and also their positive sequence. They should but are not required to consider, calculation of negative and zero sequences. Negative and zero sequence phasors of voltage and current will prepare an opportunity to consider the unbalances of the system and use that type of information for the evaluation. Unbalanced situations are due to the unsymmetrical load condition of the microgrid or unsymmetrical islanding. The latter situation may occur due to the malfunction of the breaker with a wrong single phase trip or loss of link between the main grid and the microgrid due to a physical disconnection.

\subsubsection{Inter-microgrid Synchronized Measurements}

In ordinary methods, PMUs are located on both sides, which are on the main grid side and one on the DG or PCC side (Figure 10.1).

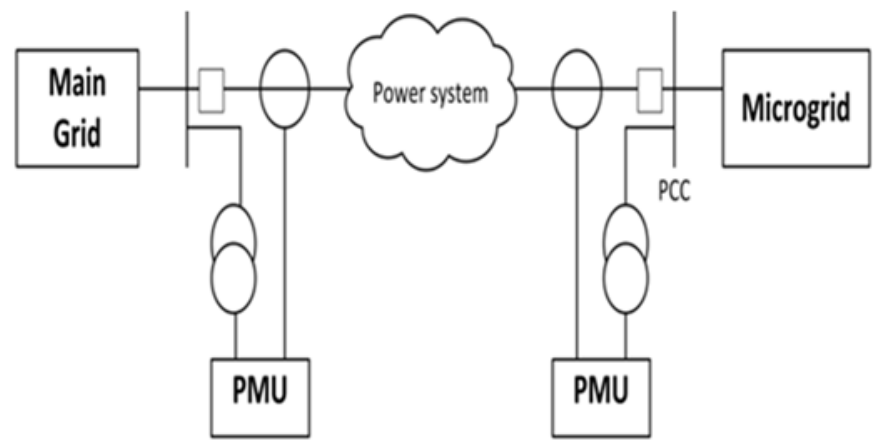

Figure 10.1 Islanding detection with PMUs located on both main grid and microgrid 
These use frequency difference method or the change of angle difference method. They compare the values on both sides during a certain minimum time of abnormal condition (difference between phases and frequencies) to be considered as islanding situation.

The frequency difference method can be represented as:

$$
\begin{aligned}
& \left|f_{D G}-f_{\text {ref }}\right|>f_{\text {threshold }} \\
& T\left(\left|f_{D G}-f_{\text {ref }}\right|>f_{\text {threshold }}\right)>T_{\text {threshold }}
\end{aligned}
$$

In which ref represents the main grid side and $D G$ for the microgrid side and $T$ represents the time duration. Whenever both differences reach the Threshold it will be considered as islanding.

The phase angle difference method is considers the changes between phases of microgrid side during islanding period. It is demonstrated as the following:

$$
\begin{aligned}
& \left|\theta_{D G}-\theta_{\text {ref }}\right|>\theta_{\text {threshold }} \\
& T\left(\left|\theta_{D G}-\theta_{\text {ref }}\right|>\theta_{\text {threshold }}\right)>T_{\text {threshold }}
\end{aligned}
$$

Threshold values are selected based on the properties of the microgrid and main grid. Some references have chosen 30 or 60 degrees for phase difference and 20 mili $\mathrm{Hz}, 0.5$ or $1 \mathrm{~Hz}$ for frequency drift [11], [49], [54]. Time period of islanding (Tthreshold) discovery is 3 seconds. In case that frequency drift exceed $1 \mathrm{~Hz}$, the islanding trip will be considered right away.

The technique which is introduced as inter microgrid synchronized measurements, is complementary to the previous methods. It uses the advantage of synchronization 
within the microgrid in a way that when the relay at microgrid side is out of service (due to any reason such as malfunction of measurement system or vulnerability by GPS jamming at the substation or DG location) other PMUs will be taken into consideration for islanding detection (Figure 10.2). By using state estimation techniques it is possible to identify the bad data and replace those values with pseudo measurements and finally detect the islanding condition.

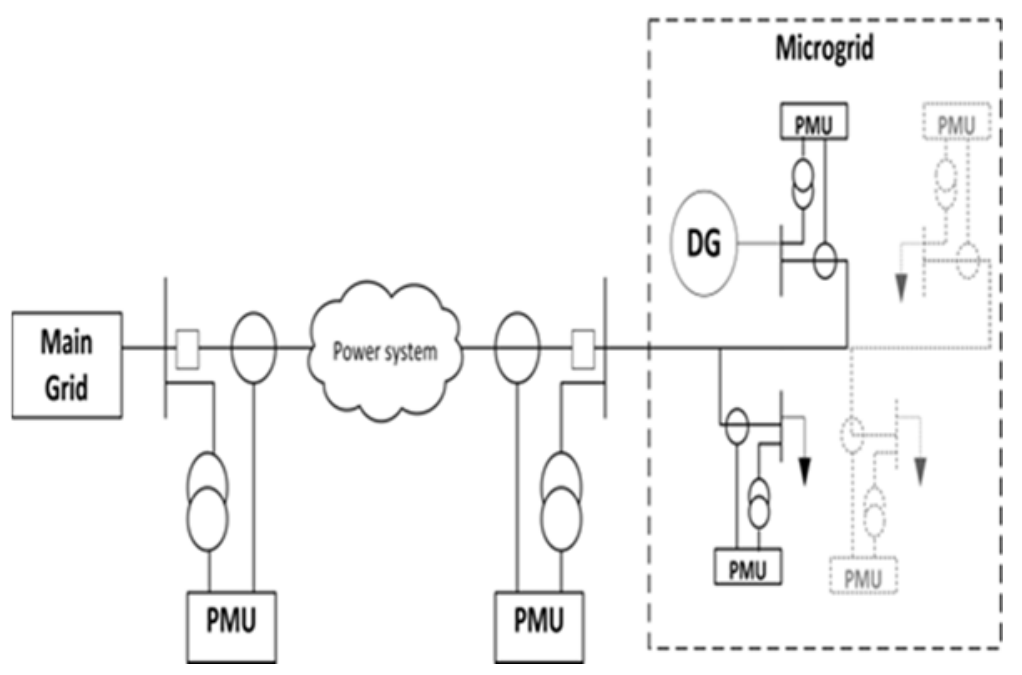

Figure 10.2 Islanding detection with other PMUs within the microgrid

\subsection{Simulation Tests}

A microgrid simulation model is built under Matlab/Simulink environment to evaluate system performance of PMUs into laboratory based microgrid electricity network (Figure 10.3). In order to have a comparison between the simple frequency reference or the phase reference and others it is assumed that they have the same case study. The parameters of synchronous generation stations are represented in table 1 . In the simulation model, Phase Locked Loop (PLL) based phase angle capturing blocks are 
implemented on both side of the transmission line connected to the distributed generation.

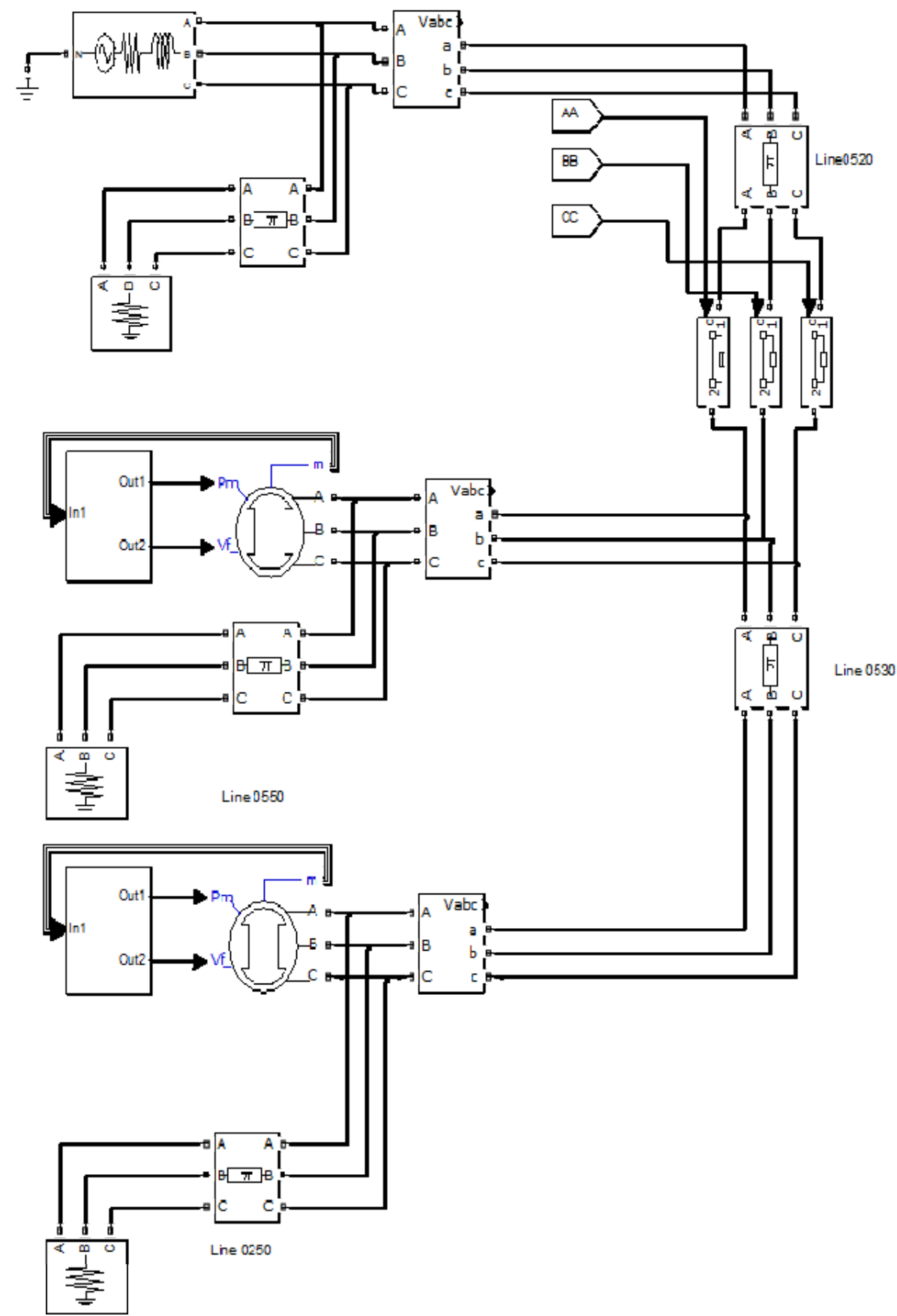

Figure 10.3 Microgrid simulation in Matlab Simulink 
Table 10.1 Generation stations parameters

\begin{tabular}{|l|l|}
\hline Parameter & Value \\
\hline Nominal Power [Sout] & $13.8 \mathrm{KVA}$ \\
\hline Line/Line voltage [VLL] & $208 \mathrm{~V}$ \\
\hline Reactance $[\mathrm{X}, \mathrm{Xd}$ ',Xd",Xq,Xq',Xq"] & {$[2.21,0.23,0.14,1.1,0.25,0.09]$} \\
\hline Time constants [Tdo'Tdo'Tq"] & {$[0.014,0.0035,0.05]$} \\
\hline Stator resistance $[\mathrm{Rs}(\mathrm{pu}]$ & 0.9 \\
\hline Inertia coefficient $[\gamma]$ & 0.3 \\
\hline
\end{tabular}

Then rate of change of frequency relay (ROCOF) implemented at the point of common coupling is used to determine system frequency and trigger the trip signal to isolate the islanding area. For the simulation purpose two scenarios have been tested and the results are presented as well.

\subsubsection{Phase and Frequency Difference}

In this scenario, the DGs are working in PV mode, $208 \mathrm{v}$ and constant power of $1.5 \mathrm{KVA}$ with a local load of $1.4 \mathrm{KW}$. Islanding is formed by opening the breaker at $\mathrm{t}=3 \mathrm{sec}$ and by evaluating the frequency at the microgrid side the frequency drift will show the changes of the frequency. In this case the generation and demand on the microgrid side does not match and it will reach the criterion of islanding detection $(\mathrm{f}=60.5 \mathrm{~Hz})$ at $\mathrm{t}=4.7 \mathrm{sec}$ (Figure 10.4). It is obvious that higher unbalance between generation and demand within the islanded area, will intensify the frequency drift. 


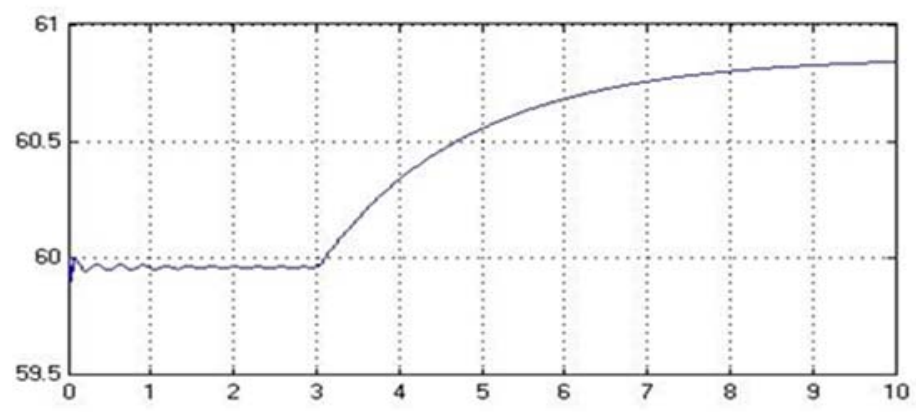

Figure 10.4 Islanding detection with PMUs between main grid and microgrid

Figure 10.5 is representing the phase difference between the grid side and the microgrid.

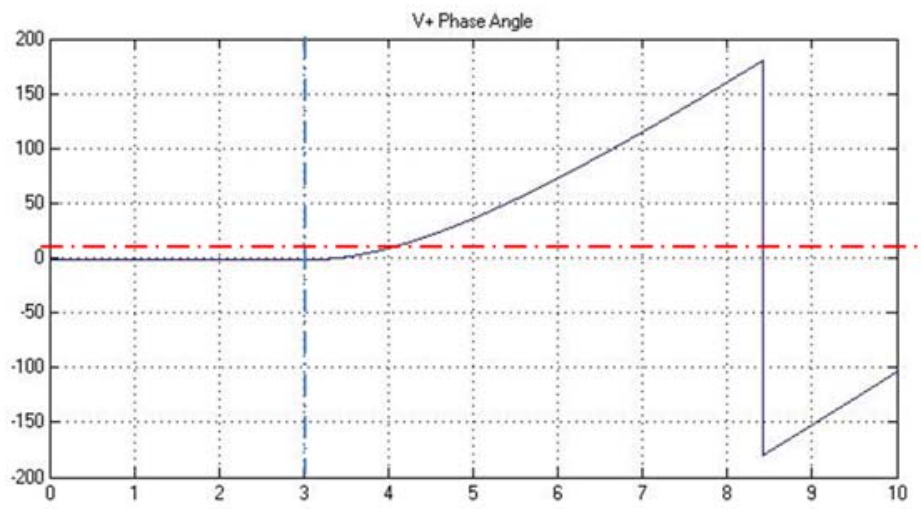

Figure 10.5 Phase angle change during islanding

According to the study the phase angle difference method gives better results in correct definition of islanding detection. ROCOF method is very sensitive to small changes in power system network such as switching events. Phase angle difference relay which is using positive sequence value has set to a 10 degree threshold to catch islanding situation. 


\subsubsection{Unbalanced Islanding of Microgrid}

The new technique for detection of unbalanced islanding situation was evaluated on the same system as in case 1 . In this case, phase "a" is disconnected at $T=3 \sec$ and phase angles and RMS values of all 3 phases of voltage to ground, and sequential vectors was demonstrated in Figure 10.6.

In this case the ROCOF relay, and positive sequence phase and frequency are not capable of detecting islanding situation correctly since their detection criterion has not met.

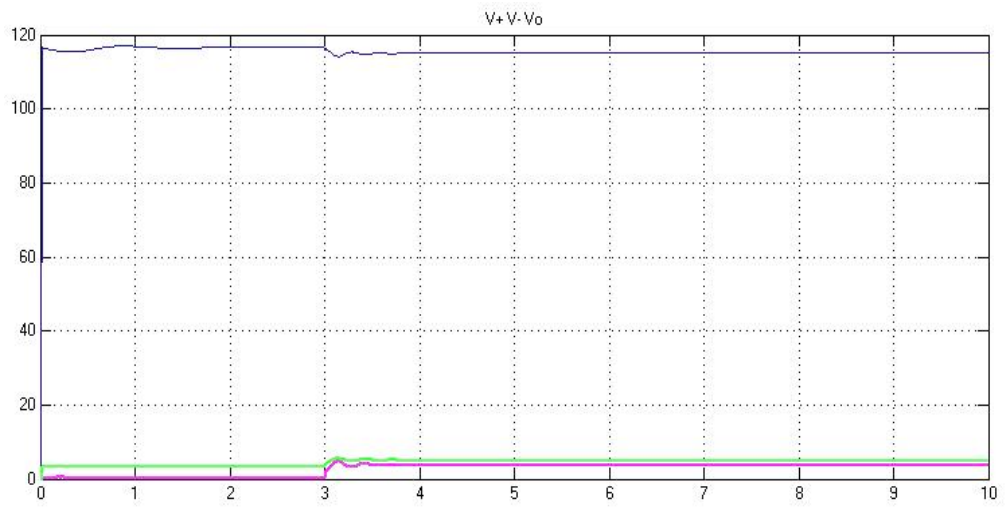

Figure 10.6 Changes of sequential RMS values in microgrid

In order to simulate the capabilities of synchronized measurements within the microgrid for islanding detection. The measurements of the other parts of the microgrid have been taken into consideration. In this scenario, the measurements of main DG or PCC has not been used for identification of islanding situation.

\subsection{Experimental Tests}

The experimental tests were performed on the Smart Grid Test Bed at Florida International University which is reconfigured for the scenarios which is briefly 
explained in the followings. It is rearrange for a 3 -phase $60 \mathrm{~Hz}, 27 \mathrm{kVA}$ system, including 2 AC generation units, 5 buses and multiple transmission line models in addition to the load emulators which are described in chapter 2. Data acquisition systems will capture the real-time data and allocated actual PMUs will gather more information from various parts of the grid. All the information was gathered for the proposed algorithm on a central computer to form the real-time SCADA system enhanced with synchronized measurements capability. Transmission lines which are used in the experimental test has well-known PI model and the parameters are included in table 2.

Table 10.2 Transmission line parameters

\begin{tabular}{|c|c|c|c|c|}
\hline Line \# & $\boldsymbol{R}$ & $\boldsymbol{X}_{\boldsymbol{L}}$ & $\boldsymbol{G}$ & $\boldsymbol{B}$ \\
\hline 520 & 0.159 & 1.614 & $62.4 \mathrm{e}-6$ & $2.53 \mathrm{e}-3$ \\
\hline 530 & 0.160 & 1.376 & $61.3 \mathrm{e}-6$ & $2.52 \mathrm{e}-3$ \\
\hline 540 & 0.160 & 1.460 & $61.3 \mathrm{e}-6$ & $2.51 \mathrm{e}-3$ \\
\hline 550 & 0.191 & 1.757 & $61.3 \mathrm{e}-6$ & $2.50 \mathrm{e}-3$ \\
\hline 250 & 0.329 & 0.464 & $50.4 \mathrm{e}-6$ & $1.70 \mathrm{e}-3$ \\
\hline
\end{tabular}




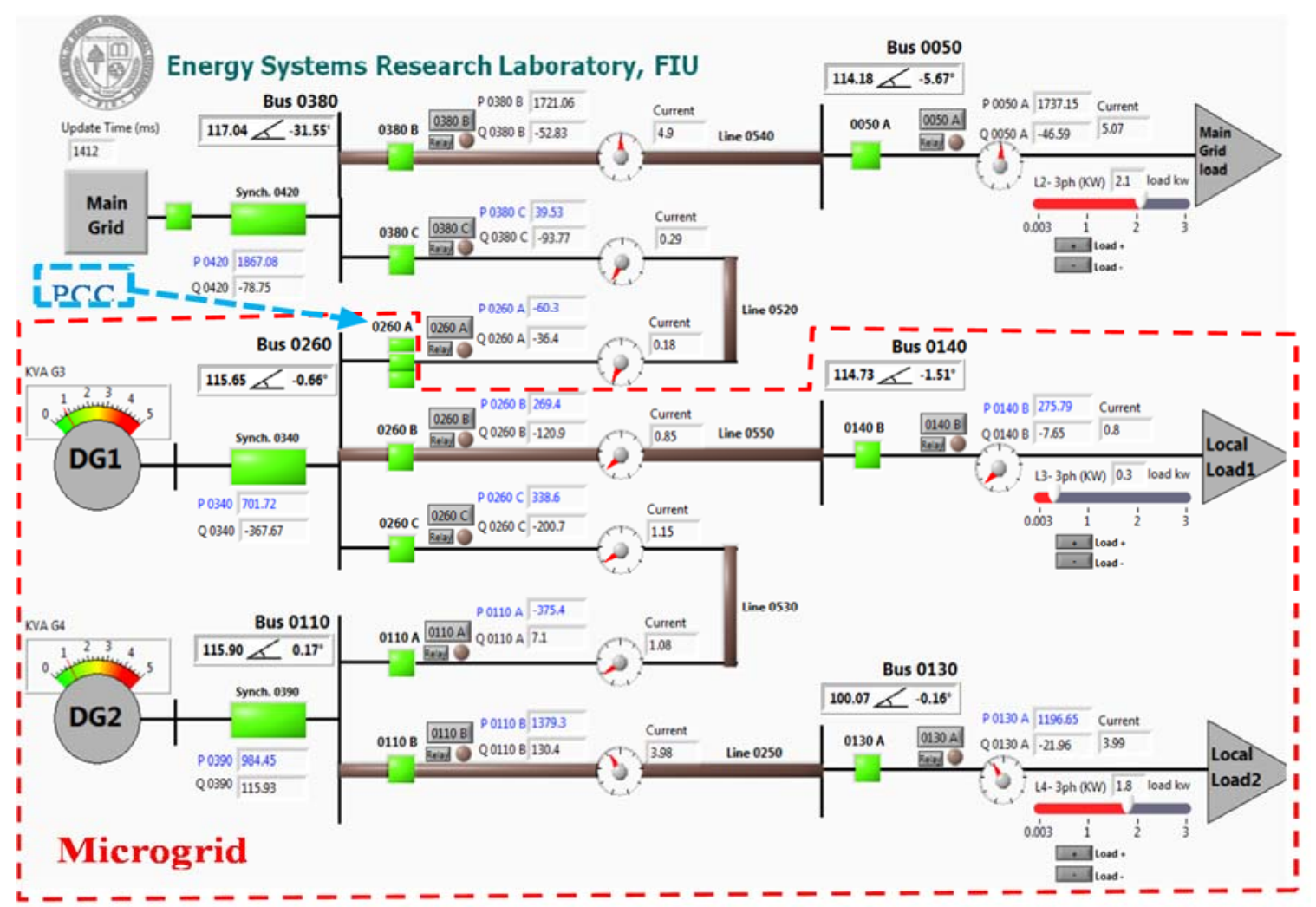

Figure 10.7 Front panel of experimental test setup

\subsubsection{Phase and Frequency Difference}

Experimental results for the test setup regarding the 3 phase islanding case is depicted in Figure 10.7. The experimental results justify the application of this method. In this case the total generation for DG units were $1470 \mathrm{w}$ and 250 Var. Total load at the microgrid side was 1395 w. Figure 10.8 is demonstrating the results. 


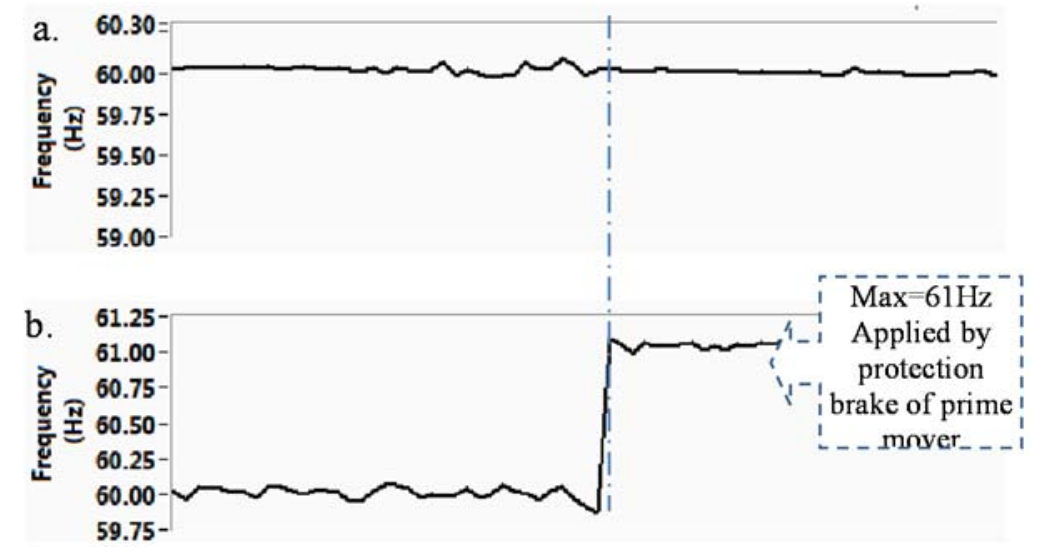

Figure 10.8 Experimental results for 3-phase islanding (Time window is 60 secs.)
a) Bus 0380
b) Bus 0260

\subsubsection{Unbalanced Islanding of Microgrid}

In this scenario, phase "a" at feeder $0260 \mathrm{~A}$ will be disconnected, and create an unbalanced islanding situation. Generations of DG1 and DG2 are $0 \mathrm{~W}$ and $1253 \mathrm{~W}$ and the loads are 268 and $888 \mathrm{~W}$. The grid has $262 \mathrm{~W}$ load at PCC and supplying $273 \mathrm{~W}$ and 97 VAR through bus 0380 .

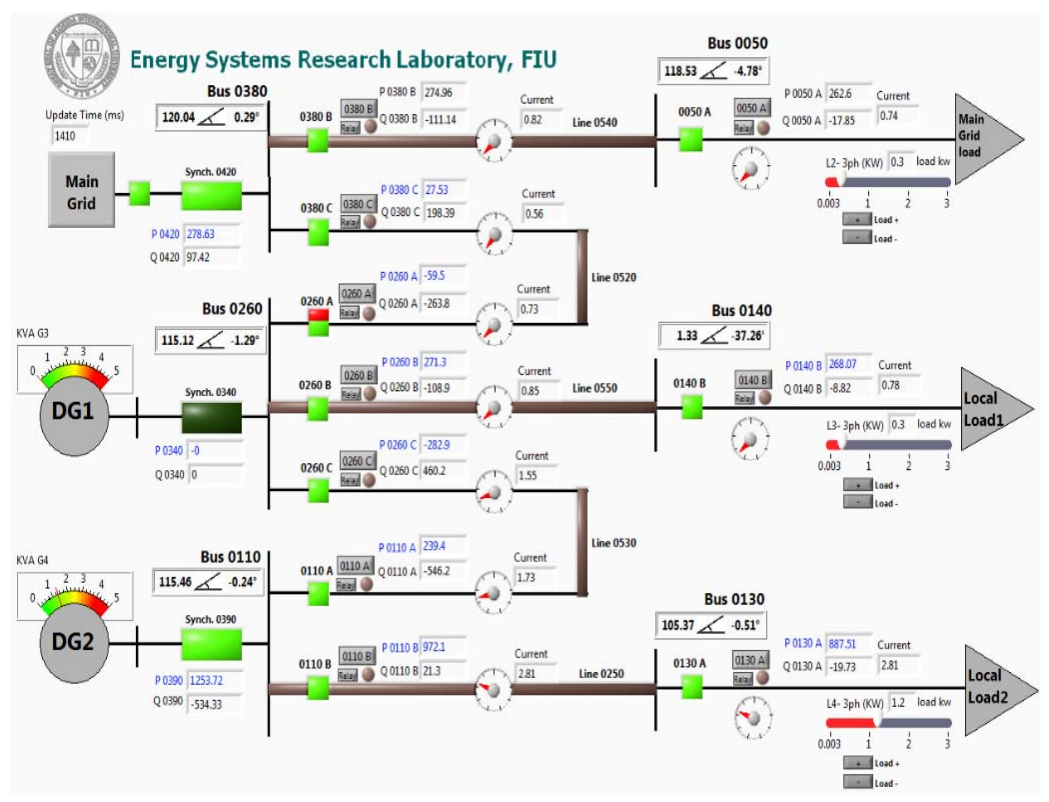

Figure 10.9 Unbalanced islanding at feeder 0260A 
The unbalanced islanding scheme and the front panel of the particular SCADA is demonstrated in Figure 10.9.

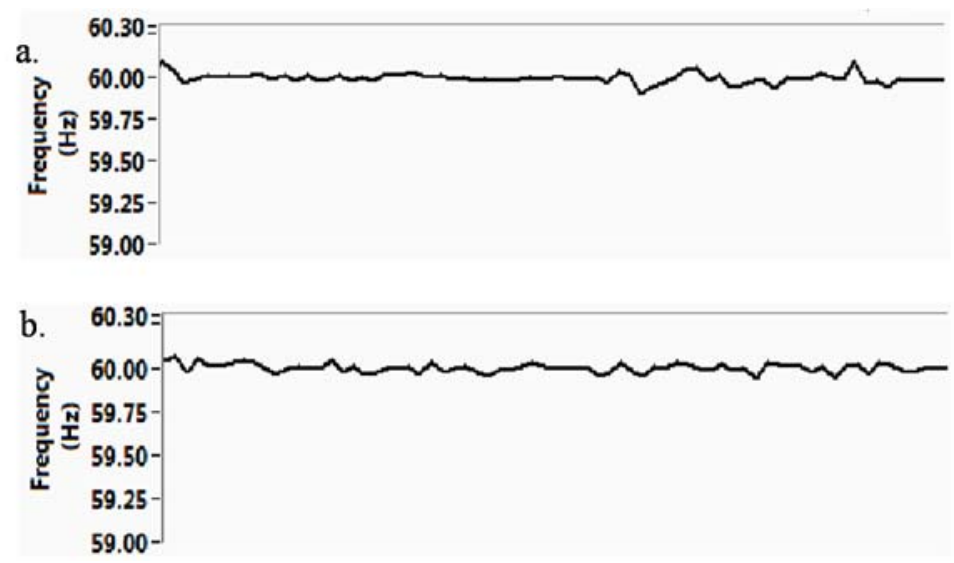

Figure 10.10 Changes of frequency during unbalanced islanding (Time window is 60 Secs.)
a) Bus 0380
b) Bus 0260

Figure 10.10 is showing the frequency of main grid and microgrid. It can be seen that in this case the traditional frequency tracking methods are not applicable.

Figure 10.11 is representing the RMS and angles of positive, negative and zero sequence. As it can be seen, the difference between before islanding and after islanding justifies the capability of this method in islanding detection. It is also indicating that positive sequence is not good enough to notice the islanding situation. Another observation was in the case where microgrid contains unbalanced loads, both sides of PCC will have the same sequential vectors, and the difference will be still the same. Hence, this technique can compare the vectors of the islanded microgrid and the main grid, conclude the islanding within the first measurement interval. 

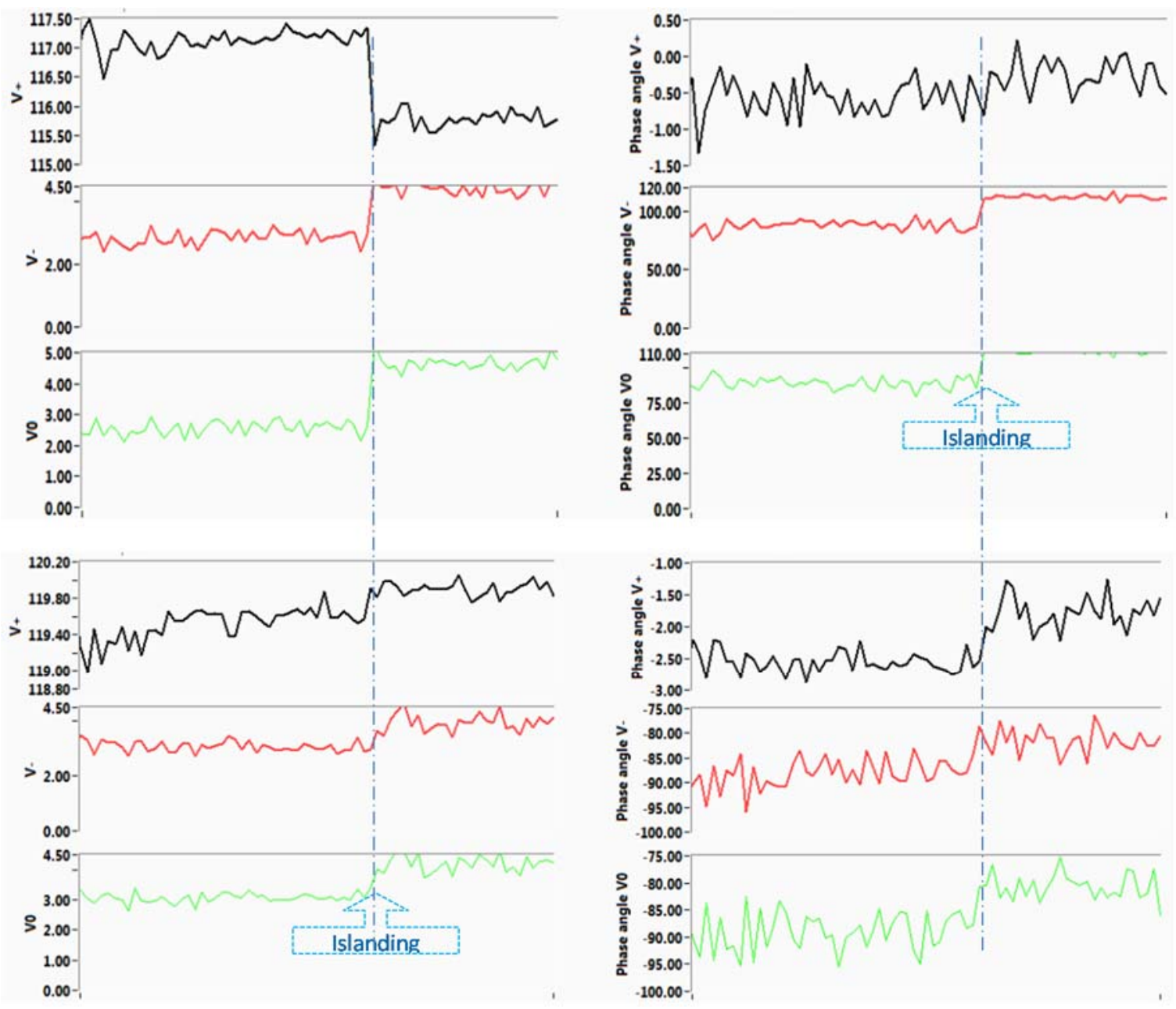

Figure 10.11 Comparing sequential RMS and angles at bus 0380 and 0260

\subsection{Conclusion}

Recent energy demands urged more integration of renewable energy resources and within sets of microgrid architectures. Improper connectivity of such microgrids to the main grid can be extremely hazardous for the main grid, and the components within the microgrid (e.g. generators) and standards recommend such situations to be identified and isolated as soon as possible.

In this Chapter, islanding detection techniques including synchrophasors and traditional methods for islanding detection were explained, simulated and experimentally 
tested. Using Phasor Measurement Units, we studied an approach to compare the phase at different locations within the microgrid or at the point of connection to other grids. Hence, using this process it is proved that the incorporation of the implemented technique introduced a method for islanding detection in microgrids with several PMUs.

Furthermore, a new method using synchronized measurements in a 3-phase system was conducted with simulations and experiments, and has shown its practical potential for the islanding detection. It is shown that using synchronized negative and zero sequential vectors, in addition to positive sequence, provides an opportunity to diagnose the partial (unbalanced) islanding of microgrids. 


\section{DEVELOPMENT OF A SOFT SYNCHRONIZATION TECHNIQUE FOR DISTRIBUTED GENERATING STATIONS}

Fast and proper synchronization of generation stations is becoming frequent, particularly in microgrids. Increased number of micro generation units raised total quantity of their synchronization process to their grids. This chapter provides enhancements of synchronization between an emulated power system and its power generation stations in the Smart Grid Testbed. For delivering the proper conditions in synchronization process, three methods including the duty-cycled control of torque controlled prime movers with and without local loads at the generator terminal, and the dynamic load brake controller have been discussed. Finally, experimental results for such methods in a real-time system have been compared.

\subsection{Introduction}

Microgrids are an emerging part of the smart grid market, which we believe will exhibit significant growth in the near future[141], [142]. Adding a generation unit to a microgrid is a very frequent event, therefore synchronization process is one of the most principal jobs in the daily operation of power systems [143]. Synchronizing a generator to the AC power system is important, because of huge damages, which may happen in case of improper closure of circuit breakers and the tensional vibration of the oscillating shaft of generators. This may lead to trip the corresponding breaker.

When paralleling two separate AC systems, it is necessary to have similar voltage characteristics at the moment of synchronization and on the Point of Common 
Connection (PCC). Phase angle, frequency and amplitude of the voltages at PCC should be identical on both AC systems prior to the connection. For Multi-phase systems (such as 3-phase systems), sequence of voltages should also be taken into account in order to have a proper synchronization process. Hence, these four conditions are referred to as the synchronizing criterion.

There are many examples of synchronizers, where a compelling case can be made for their deployment, including hospitals, high tech office parks, educational campuses, and industrial applications [144]. In all of these applications and many others, not mentioned above, proper synchronization can enhance power quality, reliability and security, as well as improve energy economics.

\subsection{Synchronization Criteria}

As mentioned before, in order to properly synchronize two AC systems, several characteristics of the voltages across the circuit breaker must be carefully monitored. These aspects are as the followings [145], [146]:

\subsubsection{Voltage Magnitude}

Any difference between the voltage magnitudes across the circuit breaker will cause a sudden transient current, which will result in huge VAR flow. Low voltage at the generator terminal, during synchronization, can cause stability problems, due to the weak magnetic circuit between the generator and the power system.

\subsubsection{Voltage Frequency}

If the frequencies across both sides of circuit breaker do not match at instant of closure, sudden active power flow will appear in response to the initial frequency 
difference until the system reaches to a stable common frequency. The allowable frequency difference is system specific however a general guideline would be to have the frequency difference within $0-0.1 \mathrm{~Hz}$.

\subsubsection{Phase Angle}

Another important criterion is the phase angle difference which can result in huge flow of active power in case of closing the switch. This phase angle is the difference between the zero crossings of the voltages on either side of the open circuit breaker.

\subsubsection{Voltage Sequence}

In three phase systems, another parameter that should be taken into account is the

voltage sequence. Since this parameter is not changing during the continuous operation of the system, it can be checked only once during the synchronization process and does not need continuous observation. Synchronizing two power systems with different voltage sequences is like coupling to motors with opposite direction of rotation which is one of the toughest tensions possible for the electrical and mechanical systems.

In case of closing the breaker while having incorrect voltage sequence the system components will confront severe damage and the protection system must terminate the synchronization procedure immediately.

\subsection{Controlling Synchronization Parameters}

Main generation stations such as nuclear, hydro, gas, steam and combined cycle power plants are mostly using turbine to extract fluid flow energy and act as the prime mover. They also utilize synchronous generators in order to convert it into electrical 
energy. Governor manages the input power of the turbine. Accordingly there are several parameters which can affect the Voltage characteristics.

\subsubsection{Frequency}

In a rotating system, the relevance between power, speed of rotation and torque is simply coming from (11.1):

$$
P=\tau . \omega
$$

In which $P$ is the mechanical power, $\tau$ is the Torque and $\omega$ is the speed of rotation. In a synchronous machine, relation between electrical frequency and rotational speed of the shaft is derived from Equation (11.2):

$$
F_{\text {elec }}=\frac{\omega_{\text {rot }} \cdot p}{120}
$$

In which $F_{\text {elec }}$ is frequency of electrical system, $p$ is the number of poles in synchronous machine and $\omega_{\text {rot }}$ is the speed of rotation.

Before connecting generation stations to the grid, output power of generator is zero, so there is no reverse torque on the shaft of turbine/generator except the mechanical friction torque.

In this case, equation (11.1) can be rewritten as:

$$
P_{\text {mec }}=\tau_{\text {Friction }} \cdot \omega_{\text {rot }}
$$

From (11.2) and (11.3) can be found:

$$
F_{\text {elec }}=\frac{p}{\tau_{\text {Friction } \cdot 120}} \cdot P_{m e c}
$$


Number of poles in a machine is constant, and for small variation of speed, torque of friction can be assumed constant. So it can be seen that electrical frequency of generator is proportional to turbine power.

Accordingly frequency of voltage can be controlled by the input power (Operation of Governor)

\subsubsection{Phase}

As mentioned in section II.C, the voltage phasors of both grid side and generator side should have the minimum possible phase at instance of closing the breaker. Considering grid side as the basis for phase reference, generator side should match this reference [147].

Speed of rotation for a synchronous machine is relative to frequency of its electrical circuit (according to equation 11.2), and also we know that the speed is the first derivative of phase function in time [148]. Consequently it is obvious that changing the speed in addition to change the frequency can change the relative phase between generator and grid phasors.

Variation of relative phase can be found through the difference between the frequencies of each side in a period of time:

$$
\Delta \varphi=\left(f_{\text {grid }}-f_{\text {gen. }}\right) \cdot t
$$

As a result matching the phase of generator into grid side requires a change in frequency (speed) of generator for a specific period of time.

Because of derivative characteristic of phase control, it requires faster and more precise control of rotational speed. 


\subsubsection{Amplitude}

Amplitude of output voltage in synchronous generators is proportional to the excitation field, which can be provided through power electronic devices or coupled DC generators. This is a significant advantage of synchronous machines which can split active and reactive power of the generator.

In order to synchronize properly, the synchronizer will decide on the proper time when all those criterions are within the acceptable range. All these conditions for the tested system are shown below:

$$
\begin{aligned}
& \left|V_{\text {right }}-V_{\text {left }}\right|<3 V \\
& \left|f_{\text {right }}-f_{\text {left }}\right|<0.1 \mathrm{~Hz} \\
& \left|\angle V_{R-\text { right }}-\angle V_{R-\text { left }}\right|<2 \mathrm{deg} \\
& \left|\angle V_{R S-\text { Right }}-\angle V_{R S-\text { left }}\right|<5 \mathrm{deg}
\end{aligned}
$$

$V_{\text {right }}$ and $V_{\text {left }}$ are the Voltages of right and left side of synchronizer, $f_{\text {right }}$ and $f_{\text {left }}$ $\mathrm{f}_{\text {left }}$ are the frequencies measured in voltage waveform for the right and the left side of the synchronizer, respectively.

Figure 11.1 shows the synchronization algorithm which was developed in the real-time software. 


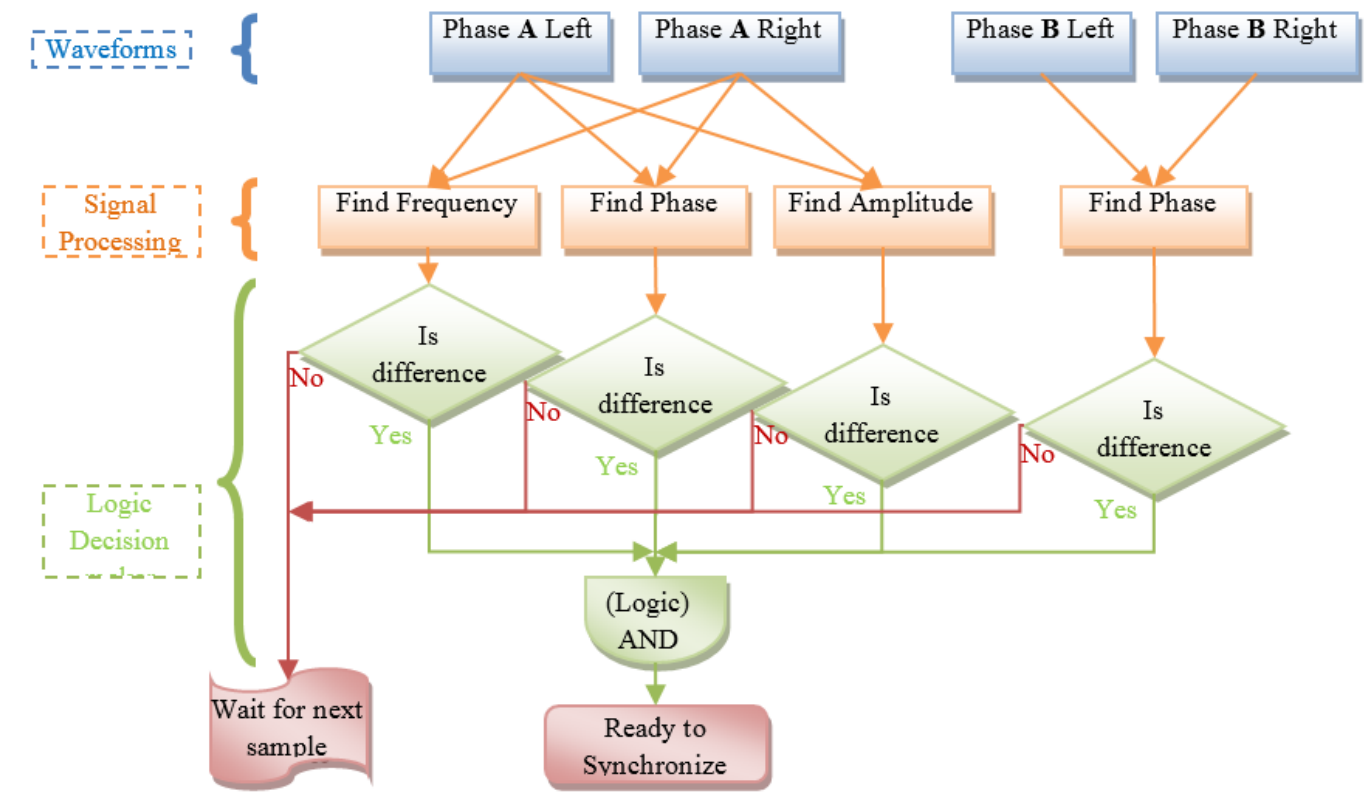

Figure 11.1 Flowchart algorithm of the synchronizer developed in real-time software

\subsection{Experimental Test Setup}

For testing and evaluation of Synchronization process and finally integration on the testbed several components were used including generation stations, transmission lines, bus models, and data acquisitions and their detailed information is available in Chapter 7.

To use generation stations as a Slack bus, the prime mover is working in frequency controlled mode to maintain grids frequency at $60 \mathrm{~Hz}$. Torque controlled mode is used to control the output active power of each generation station when connected to the grid and working at the constant speed of grid and emulating the act of PV buses. On the other hand, AVR will take care of voltage amplitude and completes emulation of a PV bus model. Using several types of data acquisition converted signals in term of time stamped waveform will be transferred to a control center. National Instrument LabVIEW 
is the software platform used to provide user friendly operation interface along with capabilities to achieve calculation, control, communication and monitoring infrastructure. The Schematic of One line diagram of the microgrid is presented in Figure 11.2.

For this experiment, the loads consist of constant impedance loads, static motor loads by using prony brake coupled to induction motors, and dynamic motor loads by using resistive load and DC Generator coupled to induction motors.

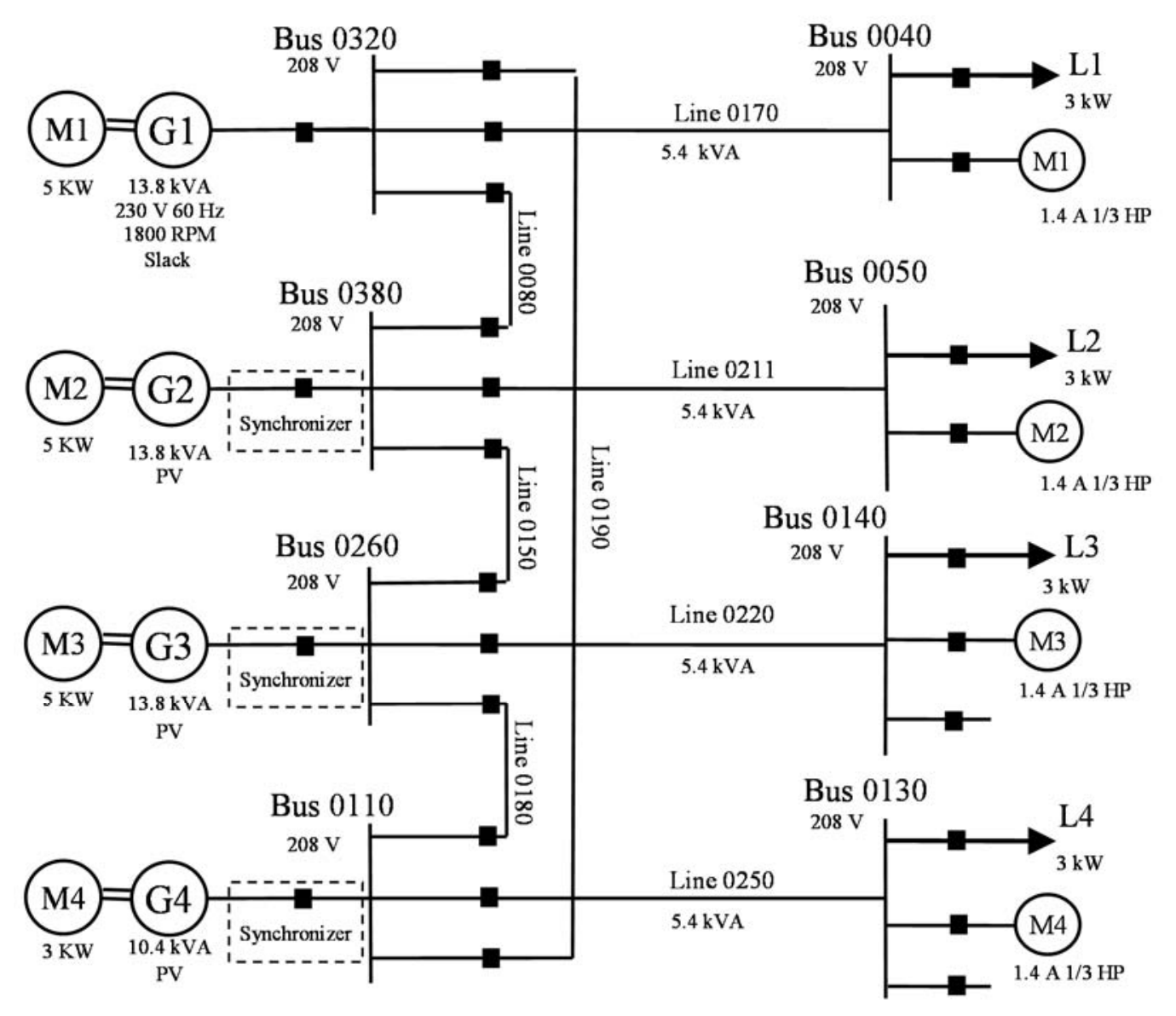

Figure 11.2 One line diagram of testing power system 


\subsection{Synchronization Strategies}

As mentioned in section 11.2, to achieve proper voltage at the point of synchronization, voltage of the generation side should be controlled to match the grid side. Since Voltage amplitude can be controlled directly and independently by the excitation system it can have an individual controller for this purpose.

Controlling the frequency as mentioned in section 11.3 has direct relevancy to the speed of rotation. Hence by changing the speed of rotation the electrical frequency of generation side can be changed.

Since the reverse torque of friction is not constant at different speeds, to control the rotational speed, it is impossible to consider torque-speed relationship completely linear. As you can see in Figure 11.3, any small changes in torque can result in large variation of speed.

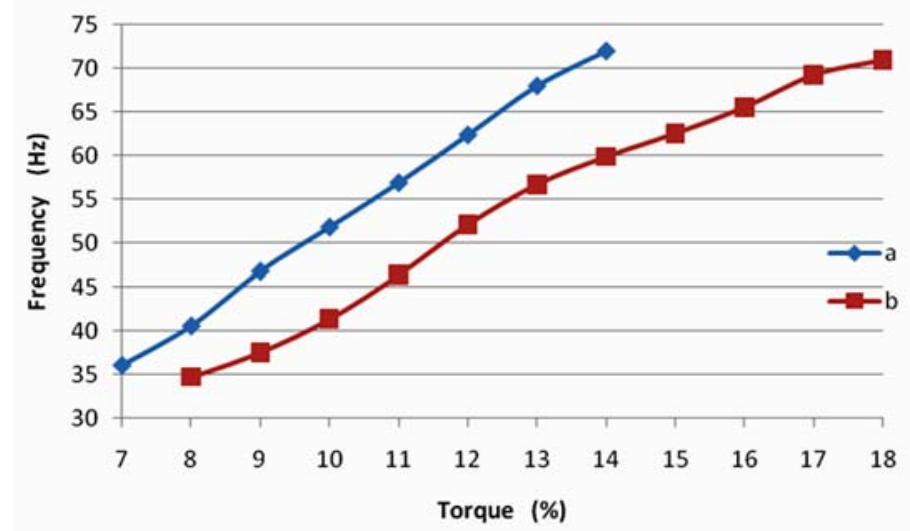

Figure 11.3 Torque-Speed curve of prime mover generator $a$. for unloaded generator $b$. with local load at the terminal of the generator

Therefore setting the speed for an unloaded prime mover-generator system requires precise control of torque. There are many situations in which this is not possible. As an example, many of these prime movers cannot set the torque precisely or they are 
working with digital controllers which cannot set the output torque in a continuous value, and there is a discrete interval between each set point.

In this study three different techniques for precise setting of torque for the purpose of controlling the rotational speed have been discussed.

\subsubsection{Duty Cycled Control}

Duty cycled controlled technique is using time intervals for changing the set point of torque control system. In this case, the set point of torque controller is changing alternatively between two values, but the time step for changing of each set point is different.

$$
\begin{gathered}
\text { Set point of controller: } \begin{cases}\Delta t_{1} & \text { High set point } \\
\Delta t_{2} & \text { Low set point }\end{cases} \\
\qquad \Delta t_{1}+\Delta t_{2}=T=\frac{1}{f_{\text {changing set point }}}
\end{gathered}
$$

This type of controller is working similar to On-Off controllers. By increasing the frequency of changing the set point $\left(f_{\text {changing set point }}\right)$ range variations for the controlled parameter (which is torque here) will decrease and better control will be provided.

New torque commands will be sent to the prime mover alternatively, and prime mover will change the torque by its internal PID controller. Schematic of Control system for this method is presented in Figure 11.4. 


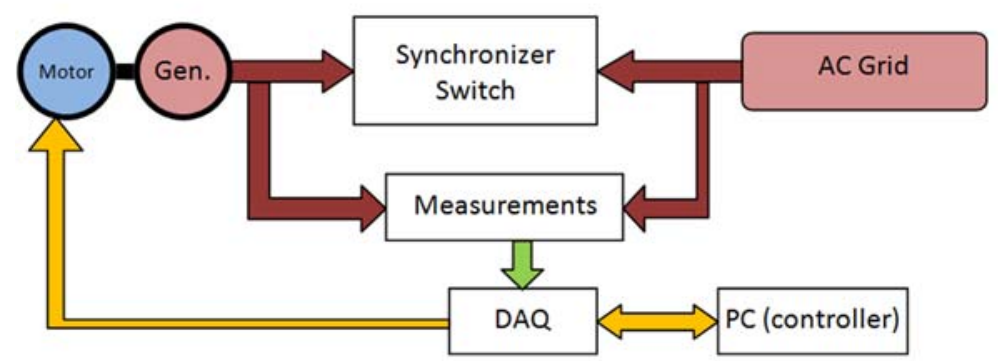

Figure 11.4 Duty cycled method, schematic of hardware connection

This method has some drawbacks as well. In the case that the communication system has a time delay, neither $\Delta t_{1}$ or $\Delta t_{2}$ should not be smaller than the communication latency. Depending on the communication limitations, it is not possible to increase the frequency of changing the set point.

In this test setup, since the prime mover (frequency drive) is using serial Modbus protocol for communication to and from the control center, it needs some silence of about 10-100 ms between each two consecutive commands depending on the communication parameters.

In addition to that, the method of data acquisition, length of sampling window, required time for waveform mathematical calculations, and time delay in communication, all will add delays to the controller and will result in an improper control of speed.

This creates limitations on frequency of changing the set point and will also affect the range of fluctuations.

Tested result for this method is shown in Figure 11.5. 


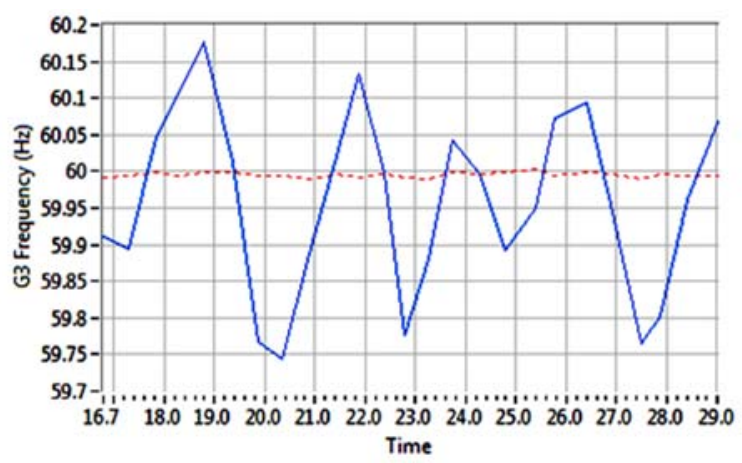

Figure 11.5 Fluctuation of generator speed in duty cycled torque controlled method (Before connecting G3 to the grid)

\subsubsection{Duty Cycled Control with Constant Local Load}

To improve the previous method, one simple approach is to put a local load at the generator terminal in order to use a larger torque of the prime mover. Since a local load will act as an opposite torque for the system, it can slow down the rotational system faster than the case in which friction is the only opposing torque. The curve of speed versus input torque is depicted in Figure 11.3. Tested results for setting the speed at the grids corresponding frequency is presented in Figure 11.6.

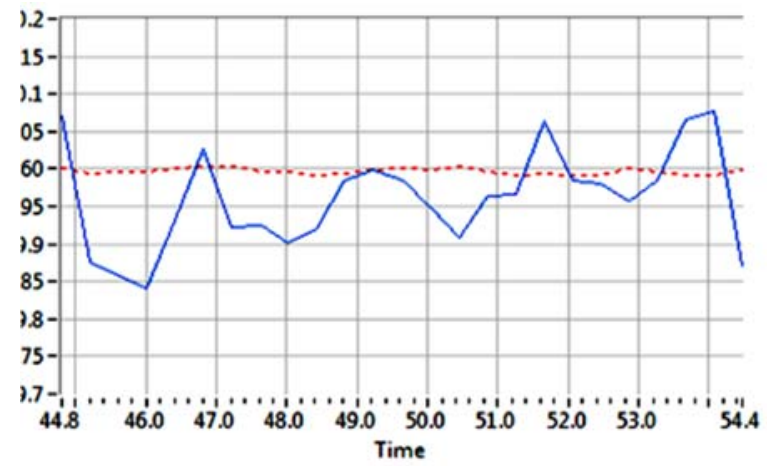

Figure 11.6 Fluctuation of generator speed in duty cycled torque controlled method when it has a local load at the $\mathrm{G} 3$ terminal (The torque is varying between 13 and 15\%) 
Although this method prepares better speed regulation, in reality it is not economical using a local load due to its increases of the amount of constant energy needed to be consumed.

\subsubsection{Dynamic Load Brake}

Another Method to set the speed of rotation to match the grids corresponding frequency, is to use a constant torque and apply a dynamic brake. This brake can be a mechanical friction type coupled to the shaft of the Prime mover/generator or a dynamic load connected to the generator terminal. Since the electrical load has better performance in terms of reliability, response time and controllability in comparison to mechanical brakes, it is more convenient to use and therefore it is discussed in this section.

The technique in which local load can regulate the speed if rotation in constant torque method will be explained hereby. Increasing the load will increase the opposing power, since the input torque is constant, according to equation (11.1):

, Speed will decrease.

By employing the dynamic load brake to the system, the schematic of the hardware connection will be as in Figure 11.7.

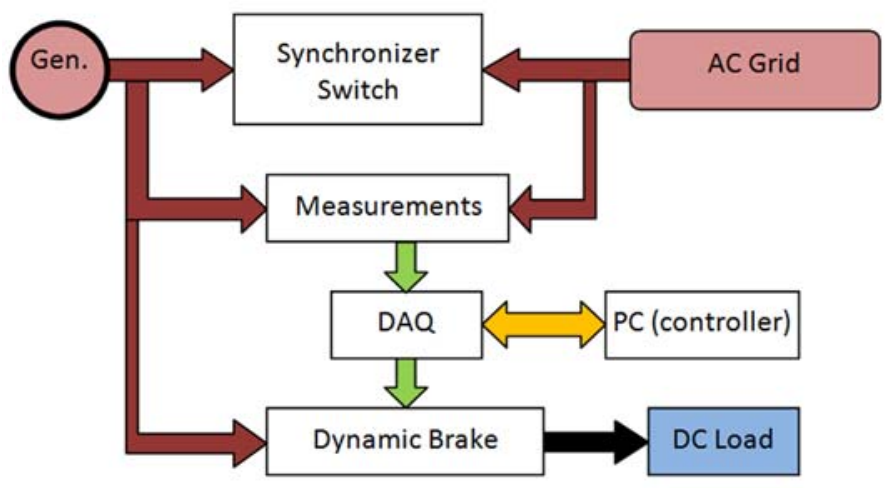

Figure 11.7 Dynamic load brake method schematic hardware connection 


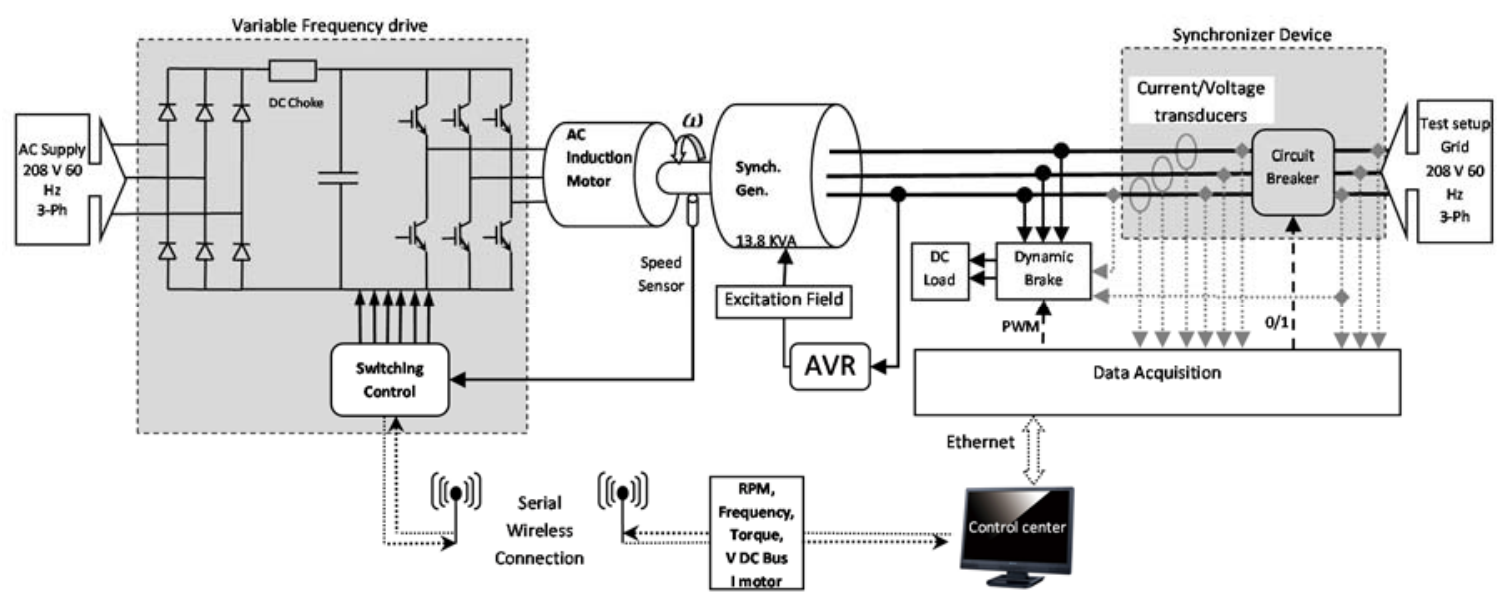

Figure 11.8 Overall schematic of the emulated generator station and its measurements, communication and controller components

According to characteristics of frequency drive which is powering the induction motor (as the prime mover), the capability to set the output torque of induction machine is in increments of $1 \%$. The provided test setup is using 3-7.5- hp Induction motors which can provide about $2-5 \mathrm{~kW}$ at each station. Assuming that the speed of rotation is constant (it should be around corresponding frequency of $60 \mathrm{~Hz}$ ), the torque and power are linearly related. So $1 \%$ of change is very close to $1 \%$ of change in output power which will be $50 \mathrm{~W}$ for a $5 \mathrm{~kW}$ induction machine. This is the maximum load required to regulate the speed when torque control is limited in steps of $1 \%$.

The dynamic load which is designed to regulate the speed is supplied from the generator terminal in 3 phases and provides the DC power for a constant resistive load. As it presented in Figure 11.9 it consists of a DC rectifier bridge, a DC voltage regulator (capacitor) and a pulse width modulation controlled switch to supply the DC load.

Designed and implemented circuit is shown in Figure 11.10. It is decided to supply the gate of IGBT switch from the same power of generator terminal and make the 
circuit self-powered, so a voltage divider and a voltage regulator is used to provide that power supply.

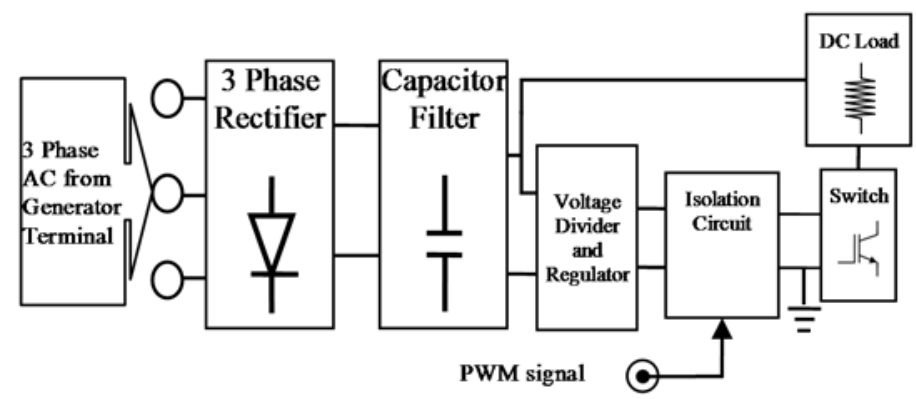

Figure 11.9 Block diagram and wiring of dynamic load

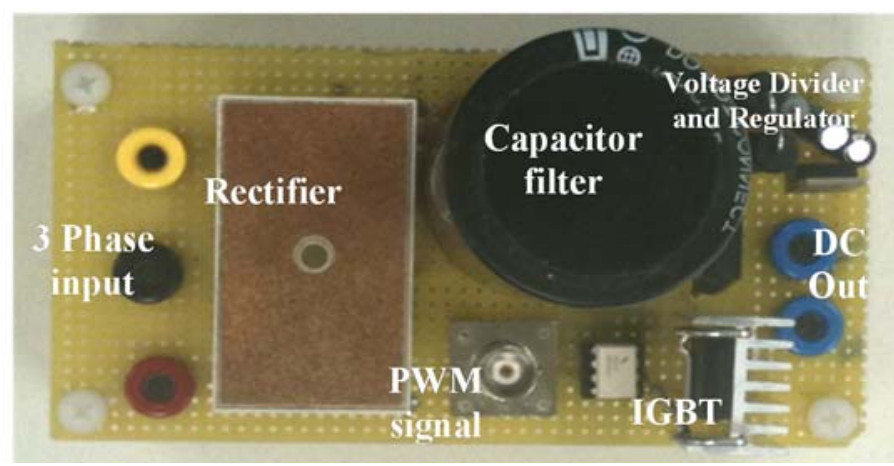

Figure 11.10 Implemented circuit for dynamic load

Dynamic brake is rectifying the AC input to DC and the IGBT will supply a DC resistive load in a PWM switching pattern, which has a variable duty cycle. Duty cycle is the parameter which can define the average amount of power to be consumed in the load.

Figure 11.11 is showing the schematic of the control loop, which has the grid frequency as the reference and take the generator frequency as the feedback, and will find the proper duty cycle of the PWM rectangular waveform.

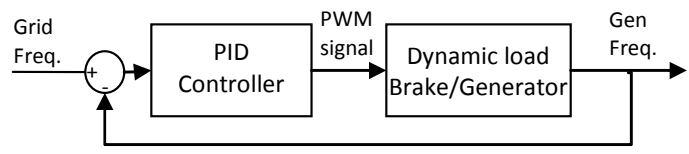

Figure 11.11 Frequency (speed) control loop by using a dynamic load brake 
By changing the frequency of generation side for specific amount of time, the phase of both sides of circuit breaker, according to equation (11.5) can be matched. This can be done by applying a new controller just for controlling the phase, after reaching to a close range of grid side frequency or it can be done by changing the set point of control diagram of Figure 11.11 in a way that reduces the phase difference.

Both methods have been tested in real-time emulated power system. Results show that in the first method, phase controller is very sensitive to the changes of duty cycle, and accordingly for each generation station a precise PID gains are required. In case that improper action of the phase controller brings the frequency out of defined range for phase controller, the control strategy will change to return the frequency to the acceptable range.

Second method for reducing the phase difference was more stable. For this method in order to change the phase, a high and low range for frequency set point $\left(f_{\text {grid }} \pm 0.1 \mathrm{~Hz}\right)$ is assumed and the setpoint will vary within that interval. The diagram of control for this method is presented in Figure 11.12.

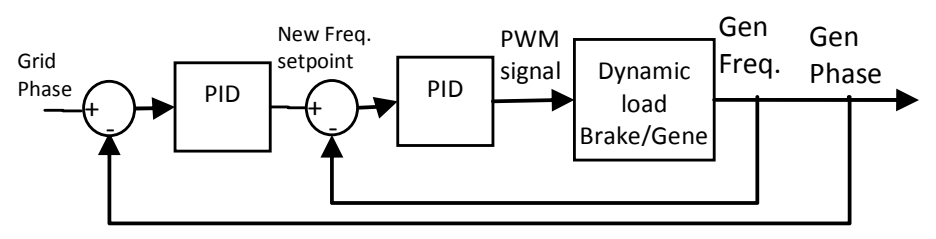

Figure 11.12 Control loop for controlling the phase by changing the frequency set point

In case that multiple number of generation units are going to be connected to the grid in same point of connection or in the same generation station, just one dynamic 
brake can be used individually with each unit, because generation unit are usually added to the system one after each other.

The front panel of dynamic load along with operational parameters is shown in Figure 11.13.

By applying the discussed methods to the power system testbed, improvements of synchronization process were significant. For explained grid, which has a slack generator and $3 \mathrm{PV}$ generation stations, full synchronization of PV stations to the grid was tested 5 times and average time required for synchronizing process are shown in table 1.

Table 11.1 Average synchronization time for each generation station for three discussed methods of controlling the frequency and phase.

\begin{tabular}{|c|c|c|c|}
\hline & Duty cycled & D.C. with load & Dynamic brake load \\
\hline G2 & 240 & 180 & 60 \\
\hline G3 & 220 & 180 & 30 \\
\hline G4 & 140 & 160 & 50 \\
\hline Total & 600 & 520 & 140 \\
\hline
\end{tabular}




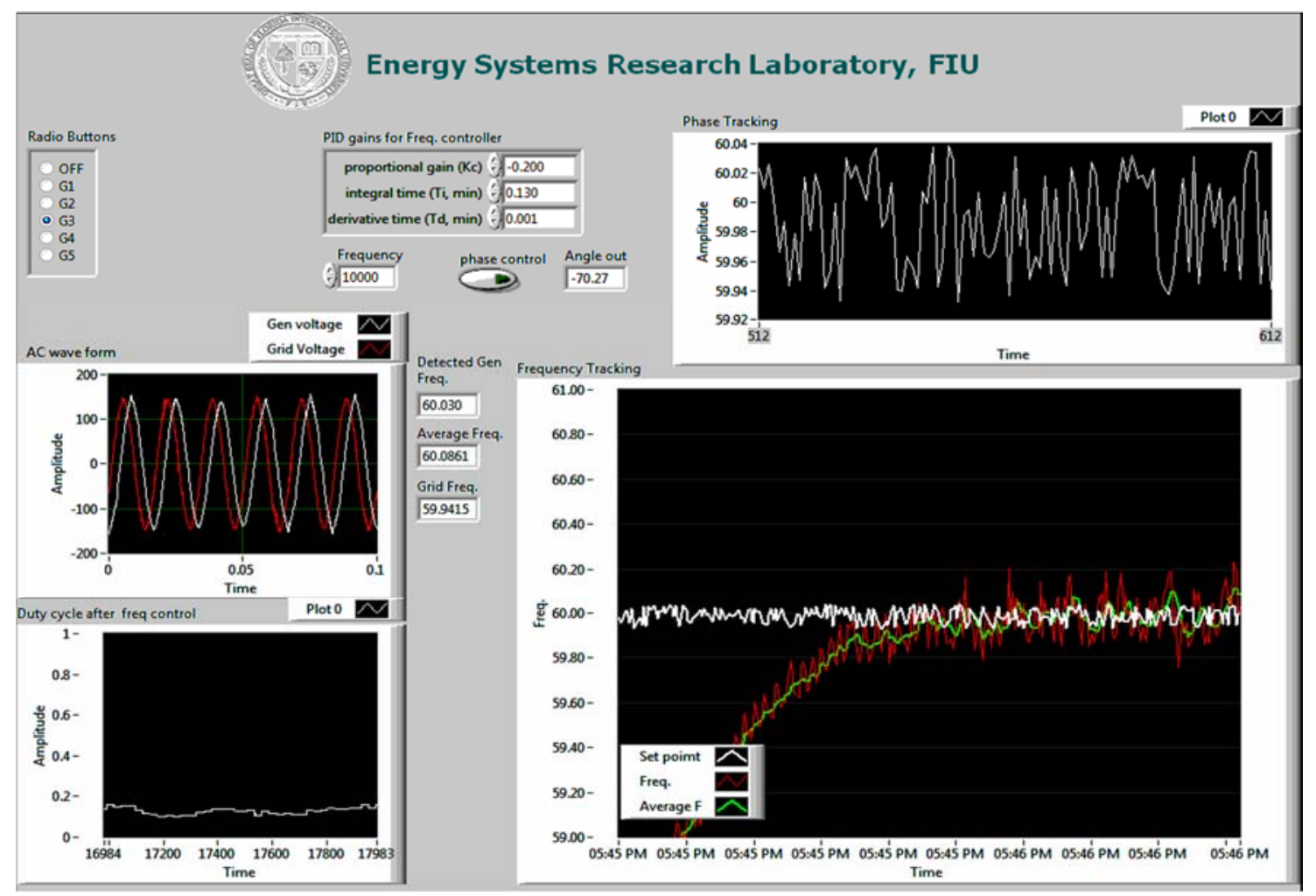

Figure 11.13 Front panel of dynamic load to match the frequency of the generator side to grid side.

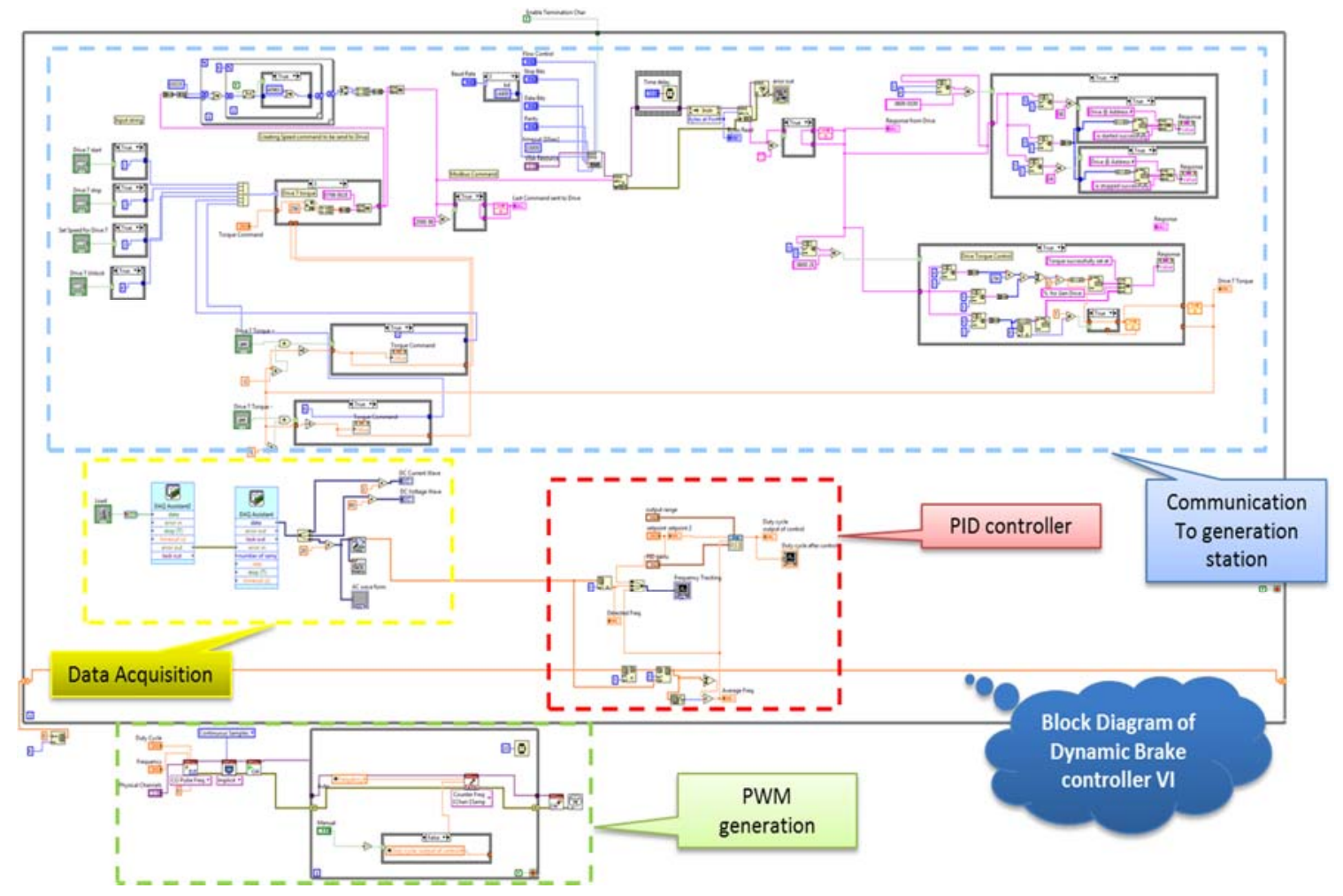

Figure 11.14 Background of dynamic brake VI 


\subsection{Conclusion}

In this chapter proper conditions for a safe synchronization of synchronous generators to power grid and the approaches to control the parameters to satisfy those conditions have been discussed. Matching the frequency and phase difference of generation side to the values of grid side is the main issue in synchronization procedure, and for such purpose three methods for controlling the speed of prime mover in constant torque mode operation of prime movers of generation stations are discussed. All methods are tested experimentally on a laboratory scaled power system and the results prove the advantages of using dynamic load brake at the terminal of each generation station. Introduced technique can reduce the total required time of synchronization to less than $25 \%$ in comparison to two other methods. 


\section{CONCLUSION AND RECOMMENDATIONS FOR FUTURE WORK}

\subsection{Conclusions}

The topics discussed in this dissertation demonstrate the difficulties encountered in monitoring, control and operation of distributed and wide area power systems by applying the new methodologies to find the state of the power system, improve the security, and identification and mitigation of several abnormal situations.

The provision for the urgent need in analyzing, planning and operation of future power grids will mostly rely on the information technology, real-time control techniques and communication infrastructure improvements.

Optimum controlling of a large system, such as a power grid, requires correct, precise, real-time and reliable information. Accordingly, this Dissertation introduces an SCADA system equipped with the developed state estimation technique from the realtime data and by incorporating a developed PMU prototype for wide area application integrity. The developed SCADA provides voltage and current waveform data and measure the power system parameters, such as the voltage and current phasors, positive, negative and zero sequences, frequency and power factor.

The developed PMU in this dissertation is open source, and flexible to be used in different topics related to synchronized measurement systems, such as security enhancement, expansion of analysis and application domain, and precision improvement.

The new device is integrated in a PDC platform along with commercial PMUs via a standard synchrophasor messaging communication, which is embedded within the 
device. By using such setup, the precision and performance of the new device was compared to a commercial PMU, and its errors in timing, phase and frequency measurements have been calculated and mitigated by calibration and according the standards. After that, a real-time technique for measuring the parameters of power transmission lines by utilizing PMU technology was introduced, applied, and verified on an experimental power system setup.

In future power systems due to large penetration of renewable energy resources and electric vehicles, formation of energy will migrate form bulk energy supplies to distributed generation within microgrids. Improper connectivity of such microgrids to the main grid should be identified and isolated as soon as possible. Hence, islanding detection techniques including synchrophasors and traditional methods for islanding detection were explained, simulated and experimentally tested afterward. Using Phasor Measurement Units, we studied an approach to compare the phase at different locations within the microgrid or at the point of connection to other grids. Simulation and experimental tests have proved that the incorporation of the alternative technique for islanding detection in microgrids with several PMUs.

Furthermore, a new method using synchronized measurements in a 3-phase system was conducted with simulations and experiments, and has shown its realistic potential for the islanding detection. It is shown that using negative and zero sequential vectors from synchronized measurements or PMUs, in addition to positive sequence, provides an opportunity to diagnose the partial (unbalanced) islanding of microgrids. 
Moreover, in this dissertation, practical implementation of a wide area state estimation was performed automatically, via a developed Matlab program, which is providing state estimation through an interconnection with LabVIEW real-time data.

By using this platform, it is possible to integrate the PMU reading for state estimation process and for special cases when a group of PMU readings are deviated from time synchronism.

In addition, we concentrated on development and implementation of a new interface to perform the S.E. and load flow on the real-time platform using PowerFactory software. The interface receives the real-time system data from the SCADA and utilizes that in the online engine mode to perform power flow and S.E. analysis. The experimental results are presented to show the capability and interoperability of the developed system.

This dissertation also proposed a trusted sensing base to protect power grid critical infrastructures against false data injection attacks. In particular, TSB deploys a synchronized and GPS-based analog data encryption (by scaling the amplitude) within sensors at the data acquisition point. Consequently, the attackers capable of control over vulnerable phasor measurement units or capable of penetrating to cyber side of A-to-D devices remain unable to manipulate acquired sensor data maliciously. The proposed technique was tested on a real-time system, including actual voltage and current reading, and PMUs. The results in real-world power system test-bed shows that TSB could efficiently reduce the size of the infrastructural trusted computing base through reducing the number of assets in power grid that need to be trusted for the whole infrastructure to give correct functionality. 
Due to the need for fast and reliable synchronization process in future power systems, we also discussed on three methods for controlling the speed of prime mover in constant torque mode operation of prime movers of generation stations. All methods are tested experimentally on a laboratory scaled power system and the results prove the advantages of using dynamic load brake at the terminal of each generation station. By utilizing the new technique in our developed dynamic brake system, we achieved $75 \%$ time reduction for synchronization of generators to the grid, compared to other methods.

This dissertation presented several techniques for enhancing the current power system infrastructure, toward a modern smart grid through and by implementing a hybrid HIL system in order to offer the most authentic mimic of power systems. This facility is provided by mimicking the $\mathrm{AC}$ assets power realistic power system, such as generation stations, synchronizers, transmission lines, measurement and switching buses, programmable loads, data acquisitions and finally the central control-analysis processor, as well as DC grid components, such as inverter-rectifier bidirectional converters, Dc loads and storage system, wind turbine emulator, and PV emulators. Integration of DC microgrids for several applications, such as real-time operation of power systems, frequency regulation of the AC power grid by DC resources, and power sharing between AC-DC microgrids was performed, and validated by experimental case studies.

The incorporation and verification of the different methodologies introduced in this work proposes a central software to be real-time, flexible and accessible for different objectives. It works as a core and should interact with control, analysis, simulation, communication, automation, database and archive, or even commercial packages within the subjects related to this dissertation. In addition, to make overall system as hardware in 
the loop, hardware interfaces should be available and compatible with this software core. Hence, a LabVIEW project was developed in a modular structure in order to be expandable, coherent, independent, reusable, and collaborative, as well as with less code and variables, and simpler procedures.

\subsection{Recommendations for Future Works}

The topics covered in this dissertation shows the challenges in real-time control and optimal operation of modern power systems. Because of essential need for understanding, recognizing and solving probable issues and obstacles of future power systems, it recommended to consider the following topics to be expanded and highlighted:

- There is a need to develop new control strategies and techniques using the phasor measurement units. Since the number of installed PMUs are increasing every day, there is a need to develop new algorithms in side area analysis and control of power systems.

- The developed smart grid testbed includes a real-time hardware-based platform of physical components in a HIL frame. It is recommended to integrate the new realtime software based emulation with physical interface to such system, in order to verify the capability of hybrid system.

- A group of PMUs in a substation are dependent on one GPS synchronization clock usually and this may be a threat for wide area applications in case they lose connectivity to satellite clock and get a deviated time source. It is recommended 
to include such sets of consistent deviated PMU data, by optimization and integrating a new objective function in the state estimation procedure.

- Future SCADA and energy management systems should be capable of incorporating PMU readings, and use them in their regular analysis and procedures, such as state estimation and optimal power flow.

- Integration of DC side PMU (a similar time-synchronized system for time stamping the DC grid events and parameters) in hybrid power system will be another recommendation, in order to be used for research and analysis purposes.

- Using AC side PMU data can also benefit the DC side controllers and operators, to manage the energy flow, and anticipate the future state of hybrid systems, before propagation into DC bus.

- Since the GPS signal is accurate with the level of micro second precision, it is recommended to integrate those for GPS synchronized encryption and authentication platforms which are widely spread and require fast response.

- Integration of PMU data, in particular frequency and reference phasor of bulk energy sources, can also be used by energy storage systems specially the super capacitors to improve their near future expectations, and be ready for propagated disturbances.

- In this chapter we investigate the TSB capabilities and applied only the scaling technique for encryption of analog signals. I is recommended to integrate to extend TSB and apply the encryption to phase and DC level of analog signals, to make it more restricted for attackers. 
- Integration of time-stamped event reporting and data logging in a platform similar to synchrophasor messaging can provide better understanding of incidents in power systems. Since most of the substations are PMU capable, it is recommended to incorporate more devices with time-stamping capabilities in future power systems.

- Our developed PMU can be manufactured and reorganized in an embedded system (involving data acquisition and communication system) and introduced to the market as an inexpensive device. This affordable device can be installed widespread and in distribution systems for monitoring and reliability purposes. 


\section{REFERENCES}

[1] Applied Cyber Security and the Smart Grid: Implementing Security Controls into the Modern Power Infrastructure.(Brief article)(Book review), vol. 28, no. 5. Ringgold, Inc, 1001.

[2] "The office of Electricity Delivery and Energy Reliability (OE)." [Online]. Available: http://energy.gov/oe/office-electricity-delivery-and-energy-reliability.

[3] "The Smart Grid: An Introduction," Department of Energy, Office of Electricity Delivery and Energy Reliability, 2007. [Online]. Available: http://energy.gov/oe/downloads/smart-grid-introduction-0.

[4] J. Hughes, "Harmonization of IEC 61970, 61968, and 61850 Models," Electr. Power Res. Inst. (EPRI), Tech. Rep, vol. 1012393, 2006.

[5] P. Zhang, F. Li, and N. Bhatt, "Next-Generation Monitoring, Analysis, and Control for the Future Smart Control Center," IEEE Trans. Smart Grid, vol. 1, no. 2, pp. 186-192, Sep. 2010.

[6] P. P. Varaiya, F. F. Wu, and J. W. Bialek, "Smart Operation of Smart Grid: RiskLimiting Dispatch," Proc. IEEE, vol. 99, no. 1, pp. 40-57, Jan. 2011.

[7] A. Molderink, V. Bakker, M. G. C. Bosman, J. L. Hurink, and G. J. M. Smit, "Management and Control of Domestic Smart Grid Technology," IEEE Trans. Smart Grid, vol. 1, no. 2, pp. 109-119, Sep. 2010.

[8] K. Moslehi and R. Kumar, "A Reliability Perspective of the Smart Grid," IEEE Trans. Smart Grid, vol. 1, no. 1, pp. 57-64, Jun. 2010.

[9] A. Bose, "Smart Transmission Grid Applications and Their Supporting Infrastructure," IEEE Trans. Smart Grid, vol. 1, no. 1, pp. 11-19, Jun. 2010.

[10] M. Liserre, T. Sauter, and J. Hung, "Future Energy Systems: Integrating Renewable Energy Sources into the Smart Power Grid Through Industrial Electronics," IEEE Ind. Electron. Mag., vol. 4, no. 1, pp. 18-37, Mar. 2010.

[11] K. Tomsovic and T. Bilke, "Power system islanding detection based on wide area measurement systems," in 2011 16th International Conference on Intelligent System Applications to Power Systems, 2011, pp. 1-6. 
[12] S. M. Amin, "Electricity infrastructure security: Toward reliable, resilient and secure cyber-physical power and energy systems," in IEEE PES General Meeting, 2010 , pp. 1-5.

[13] S. Rahman, "Smart grid expectations [In My View]," IEEE Power Energy Mag., vol. 7, no. 5, pp. 84-85, 2009.

[14] M. Shahraeini, M. H. Javidi, and M. S. Ghazizadeh, "Comparison Between Communication Infrastructures of Centralized and Decentralized Wide Area Measurement Systems," IEEE Trans. Smart Grid, vol. 2, no. 1, pp. 206-211, Mar. 2011.

[15] T. Kim, S. K. Park, and B. G. Lee, "What Is Appropriate Strategy for Smart Grid Business?: A Case Study of Test Bed in Korea," in 2010 Proceedings of the 5th International Conference on Ubiquitous Information Technologies and Applications, 2010, pp. 1-5.

[16] J. A. Momoh, "Fundamentals of analysis and computation for the Smart Grid," in IEEE PES General Meeting, 2010, pp. 1-5.

[17] J. A. Momoh, "Smart grid design for efficient and flexible power networks operation and control," in 2009 IEEE/PES Power Systems Conference and Exposition, 2009, pp. 1-8.

[18] J. De La Ree, V. Centeno, J. S. Thorp, and A. G. Phadke, "Synchronized Phasor Measurement Applications in Power Systems," IEEE Trans. Smart Grid, vol. 1, no. 1, pp. 20-27, Jun. 2010.

[19] R. Abe, H. Taoka, and D. McQuilkin, "Digital Grid: Communicative Electrical Grids of the Future," IEEE Trans. Smart Grid, vol. 2, no. 2, pp. 399-410, Jun. 2011.

[20] F. Li, W. Qiao, H. Sun, H. Wan, J. Wang, Y. Xia, Z. Xu, and P. Zhang, "Smart Transmission Grid: Vision and Framework," IEEE Trans. Smart Grid, vol. 1, no. 2, pp. 168-177, Sep. 2010.

[21] J. Medina, N. Muller, and I. Roytelman, "Demand Response and Distribution Grid Operations: Opportunities and Challenges," IEEE Trans. Smart Grid, vol. 1, no. 2, pp. 193-198, Sep. 2010.

[22] A.-H. Mohsenian-Rad, V. W. S. Wong, J. Jatskevich, R. Schober, and A. LeonGarcia, "Autonomous Demand-Side Management Based on Game-Theoretic Energy Consumption Scheduling for the Future Smart Grid," IEEE Trans. Smart Grid, vol. 1, no. 3, pp. 320-331, Dec. 2010. 
[23] K. M. Rogers, R. Klump, H. Khurana, A. A. Aquino-Lugo, and T. J. Overbye, “An Authenticated Control Framework for Distributed Voltage Support on the Smart Grid," IEEE Trans. Smart Grid, vol. 1, no. 1, pp. 40-47, Jun. 2010.

[24] Wang Juanjuan, Fu Chuang, and Zhang Yao, "Design of WAMS-Based Multiple HVDC Damping Control System," IEEE Trans. Smart Grid, vol. 2, no. 2, pp. 363374, Jun. 2011.

[25] K. Seethalekshmi, S. N. Singh, and S. C. Srivastava, "A Synchrophasor Assisted Frequency and Voltage Stability Based Load Shedding Scheme for Self-Healing of Power System," IEEE Trans. Smart Grid, vol. 2, no. 2, pp. 221-230, Jun. 2011.

[26] M. McGranaghan, D. Von Dollen, P. Myrda, and E. Gunther, "Utility experience with developing a smart grid roadmap," in 2008 IEEE Power and Energy Society General Meeting - Conversion and Delivery of Electrical Energy in the 21st Century, 2008, pp. 1-5.

[27] Y. Chen, J. Wu, Z. Ying, and S. Heng, "Application study of six-point frequency measurement method in islanding detection," in 2011 International Conference on Electrical and Control Engineering, 2011, pp. 1004-1007.

[28] A. G. Phadke and B. Kasztenny, "Synchronized Phasor and Frequency Measurement Under Transient Conditions," IEEE Trans. Power Deliv., vol. 24, no. 1, pp. 89-95, Jan. 2009.

[29] A. G. Phadke and J. S. Thorp, "Communication needs for Wide Area Measurement applications," in 2010 5th International Conference on Critical Infrastructure (CRIS), 2010, pp. 1-7.

[30] S. Horowitz, A. Phadke, and B. Renz, "The Future of Power Transmission," IEEE Power Energy Mag., vol. 8, no. 2, pp. 34-40, Mar. 2010.

[31] R. F. Nuqui and A. G. Phadke, "Phasor measurement unit placement based on incomplete observability," in IEEE Power Engineering Society Summer Meeting,, 2002, vol. 2, pp. 888-893.

[32] Z. Wu, K. Thomas, R. Sun, V. A. Centeno, and A. G. Phadke, "Three-phase instrument transformer calibration with synchronized phasor measurements," in 2012 IEEE PES Innovative Smart Grid Technologies (ISGT), 2012, pp. 1-6.

[33] J. Depablos, V. Centeno, A. G. Phadke, and M. Ingram, "Comparative testing of synchronized phasor measurement units," in IEEE Power Engineering Society General Meeting, 2004., 2004, vol. 2, pp. 948-954. 
[34] A. G. Phadke, "Synchronized phasor measurements-a historical overview," in IEEE/PES Transmission and Distribution Conference and Exhibition, vol. 1, pp. 476-479.

[35] R. F. Nuqui, A. G. Phadke, R. P. Schulz, and N. Bhatt, "Fast on-line voltage security monitoring using synchronized phasor measurements and decision trees," in 2001 IEEE Power Engineering Society Winter Meeting. Conference Proceedings (Cat. No.01CH37194), 2001, vol. 3, pp. 1347-1352.

[36] A. G. Phadke, "Synchronized phasor measurements in power systems," IEEE Comput. Appl. Power, vol. 6, no. 2, pp. 10-15, Apr. 1993.

[37] K. E. Martin, D. Hamai, M. G. Adamiak, S. Anderson, M. Begovic, G. Benmouyal, G. Brunello, J. Burger, J. Y. Cai, B. Dickerson, V. Gharpure, B. Kennedy, D. Karlsson, A. G. Phadke, J. Salj, V. Skendzic, J. Sperr, Y. Song, C. Huntley, B. Kasztenny, and E. Price, "Exploring the IEEE Standard C37.118-2005 Synchrophasors for Power Systems," IEEE Trans. Power Deliv., vol. 23, no. 4, pp. 1805-1811, Oct. 2008.

[38] R. O. Burnett, M. M. Butts, T. W. Cease, V. Centeno, G. Michel, R. J. Murphy, and A. G. Phadke, "Synchronized phasor measurements of a power system event," IEEE Trans. Power Syst., vol. 9, no. 3, pp. 1643-1650, 1994.

[39] A. G. Phadke, J. S. Thorp, and K. J. Karimi, "State Estimlatjon with Phasor Measurements," IEEE Trans. Power Syst., vol. 1, no. 1, pp. 233-238, 1986.

[40] A. G. Phadke, J. S. Thorp, R. F. Nuqui, and M. Zhou, "Recent developments in state estimation with phasor measurements," 2009 IEEE/PES Power Syst. Conf. Expo., pp. 1-7, Mar. 2009.

[41] J. S. Thorp, "Control of electric power systems using real-time measurements," IEEE Control Syst. Mag., vol. 9, no. 1, pp. 39-45, Jan. 1989.

[42] J. P. Stovall, B. J. Kirby, T. J. Overbye, J. S. Thorp, and A. G. Phadke, "Issues Associated with the Development of a Wide-Area Analysis and Visualization Environment," in Proceedings of the 39th Annual Hawaii International Conference on System Sciences (HICSS'06), 2006, vol. 10, p. 240a-240a.

[43] J. Thorp, A. Phadke, and K. Karimi, "Real time voltage-phasor measurement for static state estimation," Power Appar. Syst., vol. PAS-104, no. 11, pp. 3098-3106, 1985.

[44] A. G. Phadke and J. Thorp, "HISTORY AND APPLICATIONS OF PHASOR MEASUREMENTS," in 2006 IEEE PES Power Systems Conference and Exposition, 2006, pp. 331-335. 
[45] J. S. Thorp and A. Pal, "Dynamic state prediction based on Auto-Regressive (AR) Model using PMU data," in 2012 IEEE Power and Energy Conference at Illinois, 2012, pp. 1-5.

[46] A. Teixeira, S. Amin, H. Sandberg, K. H. Johansson, and S. S. Sastry, "Cyber security analysis of state estimators in electric power systems," in 49th IEEE Conference on Decision and Control (CDC), 2010, pp. 5991-5998.

[47] Bi. Kovarik, "History of sustainable energy." [Online]. Available: http://sustainablehistory.wordpress.com/. [Accessed: 03-Oct-2014].

[48] S. M. Kaplan and F. Sissine, Smart grid: Modernizing Electric Power Transmission and Distribution; Energy Independence, Storage and Security; Energy Independence and Security Act of 2007 (EISA); Improving Electrical Grid Efficiency, Communication, Reliability, and Resiliency; Integra. Alexandria, TheCapitol.net, 2009, p. 217.

[49] A. Timbus, A. Oudalov, and C. N. M. Ho, "Islanding detection in smart grids," in 2010 IEEE Energy Conversion Congress and Exposition, 2010, pp. 3631-3637.

[50] M. Bakhshi, R. Noroozian, and G. B. Gharehpetian, "Passive anti-islanding scheme based on reactive power in the smart grids."

[51] A. Abur, "Post disturbance island identification via state estimation," in 2012 IEEE PES Innovative Smart Grid Technologies (ISGT), 2012, pp. 1-5.

[52] A. A. Salam, A. Mohamed, and M. A. Hannan, "Technical challenges on microgrids," ARPN J. ..., vol. 3, no. 6, pp. 64-69, 2008.

[53] A. Etxegarai, P. Eguía, and I. Zamora, "Analysis of Remote Islanding Detection Methods for Distributed Resources," 2011.

[54] D. M. Laverty, D. J. Morrow, R. Best, and M. Cregan, "Anti-islanding detection using Synchrophasors and Internet Protocol telecommunications," in 2011 2nd IEEE PES International Conference and Exhibition on Innovative Smart Grid Technologies, 2011, pp. 1-5.

[55] M. Braendle, "Cyber security for power systems - A closer look at the drivers and how to best approach the new challenges," in 2011 64th Annual Conference for Protective Relay Engineers, 2011, pp. 322-327.

[56] J. T. Tengdin, "The 2008 revision of IEEE C37.2 Standard for Electrical Power System Device Function Numbers, Acronyms, and Contact Designations," in Conference Record 2009 IEEE Industrial \& Commercial Power Systems Technical Conference, 2009, pp. 1-7. 
[57] B. McMillin, "Complexities of information security in Cyber-Physical Power Systems," in 2009 IEEE/PES Power Systems Conference and Exposition, 2009, pp. $1-2$.

[58] A. K. Srivastava and R. M. Reddi, "Real time test bed development for power system operation, control and cyber security," in North American Power Symposium 2010, 2010, pp. 1-6.

[59] A. Stefanov and C.-C. Liu, "Cyber-power system security in a smart grid environment," in 2012 IEEE PES Innovative Smart Grid Technologies (ISGT), 2012, pp. 1-3.

[60] C. Beasley, G. K. Venayagamoorthy, and R. Brooks, "Cyber security evaluation of synchrophasors in a power system," in 2014 Clemson University Power Systems Conference, 2014, pp. 1-5.

[61] G. N. Ericsson, "Cyber Security and Power System Communication-Essential Parts of a Smart Grid Infrastructure," IEEE Trans. Power Deliv., vol. 25, no. 3, pp. 1501-1507, Jul. 2010.

[62] Y. Liu, P. Ning, and M. K. Reiter, "False Data Injection Attacks Against State Estimation in Electric Power Grids," ACM Trans.Inf.Syst.Secur., vol. 14, no. 1, pp. 13:1-13:33, Jun. 2011.

[63] S. A. Zonouz, K. R. Joshi, and W. H. Sanders, "Cost-aware Systemwide Intrusion Defense via Online Forensics and On-demand Detector Deployment," in Proceedings of the 3rd ACM Workshop on Assurable and Usable Security Configuration, 2010, pp. 71-74.

[64] C.-W. Ten, C.-C. Liu, and G. Manimaran, "Vulnerability Assessment of Cybersecurity for SCADA Systems," IEEE Trans. Power Syst., vol. 23, no. 4, pp. 1836-1846, Nov. 2008.

[65] Z. Mohajerani, F. Farzan, M. Jafary, Y. Lu, D. Wei, N. Kalenchits, B. Boyer, M. Muller, and P. Skare, "Cyber-related risk assessment and critical asset identification within the power grid," in IEEE PES T\&D 2010, 2010, pp. 1-4.

[66] M. Naedele, "Network Security for Substation Automation Systems," vol. 2187, 2001, p. 25; 25-34; 34.

[67] D. J. K. J. J. Lee, B. H. Kim, and D. Hur, "Proposal strategies of key management for data encryption in scada network of electric power systems," Int. J. Electr. Power, vol. 33, no. 9, pp. 1521-1526, 2011. 
[68] "IEC Smart Grid Standadization Roadmap." [Online]. Available: www.IEC.ch/smartgrid.

[69] E. W. Gunther, A. Snyder, G. Gilchrist, and D. R. Highfill, "Smart Grid Standards Assessment and Recommendations for Adoption and Development." [Online]. Available: http://www.neopanora.com/images/stories/docs/Smart Grid Standards Landscape White Paper v0 83.doc.

[70] C. King, C. S. Officer, and Em. Corporation, "Advanced Metering Infrastructure (AMI) Overview of System Features and Capabilities," 2004. [Online]. Available: http://sites.energetics.com/madri/toolbox/pdfs/background/king.pdf. [Accessed: 29-Aug-2014].

[71] R. Ma, H.-H. Chen, Y.-R. Huang, and W. Meng, "Smart Grid Communication: Its Challenges and Opportunities," IEEE Trans. Smart Grid, vol. 4, no. 1, pp. 36-46, Mar. 2013.

[72] G. Locke and P. D. Gallagher, "NIST framework and roadmap for smart grid interoperability standards, release 1.0," Natl. Inst. Stand. Technol., p. 33, 2010.

[73] "Roadmap for Smart Grid Interoperability Standards," NIST Spec. Publ., vol. 1108, 2010.

[74] M. M. Eissa, M. E. Masoud, and M. M. M. Elanwar, "A Novel Back Up Wide Area Protection Technique for Power Transmission Grids Using Phasor Measurement Unit," IEEE Trans. Power Deliv., vol. 25, no. 1, pp. 270-278, Jan. 2010.

[75] M. G. M. Zanjani and H. K. Kargar, "High impedance fault detection of distribution network by phasor measurement units." pp. 1-5, 2012.

[76] E. Farantatos, R. Huang, G. Cokkinides, and A. Meliopoulos, "Implementation of a 3-Phase State Estimation Tool Suitable for Advanced Distribution Management Systems," IEEE PSCE, pp. 1-8, 2011.

[77] A. S. Meliopoulos and F. Zhang, "Multiphase power flow and state estimation for power distribution systems," Power Syst. IEEE, vol. 11, no. 2, pp. 939-946, 1996.

[78] G. Stefopoulos, R. Alaileh, G. Cokkinides, and A. Meliopoulos, "On Three-Phase State Estimation in the Presence of GPS-Synchronized Phasor Measurements," Power Symp., 2007.

[79] R. Hoffman, "Practical state estimation for electric distribution networks," Power Syst. Conf. Expo. 2006, pp. 510-517, 2006. 
[80] S. Balasubramani, S. Raghuraman, S. Venkatesh, C. Karthick, and B. Vidya, "Algorithm for state estimation and network error identification with Phasor measurements," (ICECT), 2011 3rd, pp. 285-289, 2011.

[81] F. Schweppe, "Power System Static-State Estimation, Part III: Implementation," IEEE Trans. Power Appar. Syst., vol. PAS-89, no. 1, pp. 130-135, Jan. 1970.

[82] M. Hurtgen and J. Maun, "Advantages of power system state estimation using Phasor Measurement Units," Power Syst. Comput. Conf., pp. 1-7, 2008.

[83] D. Novosel and K. Vu, "Benefits of PMU technology for various applications," $o$ sustavu vođenja EES-a HK, pp. 1-13, 2006.

[84] J. Giri and H. Wu, "PMU Impact on State Estimation Reliability for Improved Grid Security,” 2005/2006 Pes Td, pp. 1349-1351, 2006.

[85] F. Chen, X. Han, Z. Pan, and L. Han, "State estimation model and algorithm including pmu," Electr. Util. Deregul., no. April, pp. 1097-1102, 2008.

[86] X. Dongjie, H. Renmu, W. Peng, and X. Tao, "Comparison of several PMU placement algorithms for state estimation," Dev. Power, pp. 32-35, 2004.

[87] J. Zhang, G. Welch, G. Bishop, and Z. Huang, "Optimal PMU Placement Evaluation for Power System Dynamic State Estimation,” IEEE PES Conf., 2010.

[88] Q. Li and R. Negi, "Phasor measurement units placement for power system state estimation: a greedy approach,” Power Energy Soc. Gen., pp. 1-8, 2011.

[89] S. Chakrabarti and E. Kyriakides, "PMU measurement uncertainty considerations in WLS state estimation," Power Syst. IEEE, vol. 24, no. 2, pp. 1062-1071, 2009.

[90] R. Baltensperger, A. Loosli, H. Sauvain, M. Zima, G. Andersson, and R. Nuqui, "An implementation of two-stage hybrid state estimation with limited number of PMU," 10th IET Int. Conf. Dev. Power Syst. Prot. (DPSP 2010). Manag. Chang., pp. 1-5, 2010.

[91] S. Skok, I. Pavic, A. Barta, I. Ivankovic, N. Baranovic, Z. Cerina, and R. Matica, "Hybrid State Estimation Model Based on PMU and SCADA Measurements," Monit. Power Syst. Dyn. Perform., pp. 1-8, 2008.

[92] C. Martinez, M. Parashar, J. Dyer, and J. Coroas, "Phasor Data Requirements for Real Time Wide Area Monitoring, Control \& Protection Applications," in EIPP white paper, 2005. 
[93] A. Abur and A. G. Expósito, Power System State Estimation: Theory and Implementation. CRC Press, 2004.

[94] R. F. Jeffers, "Techniques for Wide-Area State Estimation in Power Systems," Virginia TEch., 2006.

[95] Falliere, Nicolas, L. O. Murchu, and E. Chien., "W32. stuxnet dossier," White paper, Symantec Corp., Security Response, 2011.

[96] R. Langner, "Stuxnet: Dissecting a Cyberwarfare Weapon," IEEE Secur. Priv. Mag., vol. 9, no. 3, pp. 49-51, May 2011.

[97] E. Handschin, F. C. Schweppe, J. Kohlas, and A. Fiechter, "Bad data analysis for power system state estimation," IEEE Trans. Power Appar. Syst., vol. 94, no. 2, pp. 329-337, Mar. 1975.

[98] A. Monticelli, F. F. Wu, and M. Yen, "Multiple Bad Data Identification for State Estimation by Combinatorial Optimization," IEEE Power Eng. Rev., vol. PER-6, no. 7, pp. 73-74, Jul. 1986.

[99] S. Zonouz, K. M. Rogers, R. Berthier, R. B. Bobba, W. H. Sanders, and T. J. Overbye, "SCPSE: Security-Oriented Cyber-Physical State Estimation for Power Grid Critical Infrastructures," IEEE Trans. Smart Grid, vol. 3, no. 4, pp. 17901799, Dec. 2012.

[100] B. Kauer, "Oslo: Improving the security of trusted computing," Proc. 16th USENIX Secur. Symp. USENIX Secur. Symp. SS.

[101] T. Garfinkel, B. Pfaff, J. Chow, M. Rosenblum, and D. Boneh, "Terra: A virtual machine-based platform for trusted computing," in SOSP'03: Proceedings of the 19th ACM Symposium on Operating Systems Principles, October 19, 2003 October 22, 2003, vol. 37, pp. 193-206.

[102] "Timing Committee, Telecommunications \& Timing Group, RCC, WSMR, NM, IRIG Standard 200-04 IRIG Serial Time Code Formats,” 2004.

[103] N. Hatziargyriou, H. Asano, R. Iravani, and C. Marnay, "Microgrids," IEEE Power Energy Mag., vol. 5, no. 4, pp. 78-94, Jul. 2007.

[104] "SX460 AUTOMATIC VOLTAGE REGULATOR (AVR) SPECIFICATION, INSTALLATION AND ADJUSTMENTS," Newage International Limited, 2005. [Online]. Available: www.newage-avkseg.com.

[105] A. Mohamed, C. F. de Cossio, T. Ma, M. Farhadi, and O. Mohammed, "Operation and protection of photovoltaic systems in hybrid AC/DC smart grids," in IECON 
2012-38th Annual Conference on IEEE Industrial Electronics Society, 2012, pp. 1104-1109.

[106] M. M. Amin and O. A. Mohammed, "Development of High-Performance GridConnected Wind Energy Conversion System for Optimum Utilization of Variable Speed Wind Turbines," IEEE Trans. Sustain. Energy, vol. 2, no. 3, pp. 235-245, Jul. 2011.

[107] O. A. Jaramillo and M. A. Borja, "Wind speed analysis in La Ventosa, Mexico: a bimodal probability distribution case," Renew. Energy, vol. 29, no. 10, pp. 16131630, Aug. 2004.

[108] D. Kottick, M. Blau, and D. Edelstein, "Battery energy storage for frequency regulation in an island power system," IEEE Trans. Energy Convers., vol. 8, no. 3, pp. 455-459, 1993.

[109] Q. Zhou and D. Yuan, "Prediction on future DC power system," in 2009 IEEE 6th International Power Electronics and Motion Control Conference, 2009, pp. 11921195.

[110] A. K. Chakravorti and A. E. Emanuel, "Design, analysis and limitations of a DCto-AC converter usable for interface alternative energy sources and energy storage systems with the utility grid," in Proceedings of IEEE Power Electronics Specialist Conference - PESC '93, pp. 595-601.

[111] G. C. Zweigle and V. Venkatasubramanian, "Wide-Area Optimal Control of Electric Power Systems With Application to Transient Stability for Higher Order Contingencies," IEEE Trans. Power Syst., vol. 28, no. 3, pp. 2313-2320, Aug. 2013.

[112] J. Ning, X. Pan, and V. Venkatasubramanian, "Oscillation modal analysis from ambient synchrophasor data using distributed frequency domain optimization," IEEE Trans. Power Syst., vol. 28, no. 2, pp. 1960-1968, May 2013.

[113] A. von Meier, D. Culler, A. McEachern, and R. Arghandeh, "Microsynchrophasors for distribution systems," IEEE Innov. Smart Grid Technol. (ISGT 2013), 2013.

[114] A. Borghetti, C. A. Nucci, M. Paolone, G. Ciappi, and A. Solari, "Synchronized Phasors Monitoring During the Islanding Maneuver of an Active Distribution Network," IEEE Trans. Smart Grid, vol. 2, no. 1, pp. 82-91, Mar. 2011.

[115] R. Garcia-Valle, G.-Y. Yang, K. E. Martin, A. H. Nielsen, and J. Ostergaard, "DTU PMU laboratory development - Testing and validation," in 2010 IEEE 
PES Innovative Smart Grid Technologies Conference Europe (ISGT Europe), 2010, pp. 1-6.

[116] Q. B. Dam, S. Mohagheghi, R. H. Alaileh, G. K. Stefopoulos, G. J. Cokkinides, and A. P. S. Meliopoulos, "A laboratory setup for relay and GPS-synchronized equipment transient testing," in 2008 IEEE Power and Energy Society General Meeting - Conversion and Delivery of Electrical Energy in the 21st Century, 2008, pp. $1-8$.

[117] A. P. S. Meliopoulos, G. J. Cokkinides, S. Mohagheghi, Q. B. Dam, R. H. Alaileh, and G. K. Stefopoulos, "A laboratory setup of a power system scaled model for testing and validation of EMS applications," in 2009 IEEE Bucharest PowerTech, 2009, pp. 1-8.

[118] K. Zhu, S. Deo, A. T. Al-Hammouri, N. Honeth, M. Chenine, D. Babazadeh, and L. Nordstrom, "Test platform for synchrophasor based wide-area monitoring and control applications," in 2013 IEEE Power \& Energy Society General Meeting, 2013, pp. 1-5.

[119] B. Vandiver, A. Apostolov, and F. Steinhauser, "Testing of Phasor Measurement Units," in 2010 63rd Annual Conference for Protective Relay Engineers, 2010, pp. $1-5$.

[120] Y. Yu, D. Zhang, Z. Zhou, J. Zhou, and T. Lu, "The method for Synchronized Phasor Measurement Unit testing and evaluation," in 2011 International Conference on Advanced Power System Automation and Protection, 2011, vol. 3, pp. 2206-2212.

[121] A. Nechifor, P. Regulski, D. Cai, and V. Terzija, "Development of a flexible laboratory testing platform for assessing steady-state and transient performance of WAMS," in 2011 IEEE International Workshop on Applied Measurements for Power Systems (AMPS), 2011, pp. 62-67.

[122] L. Vanfretti, V. H. Aarstrand, M. S. Almas, V. S. Peric, and J. O. Gjerde, "A software development toolkit for real-time synchrophasor applications," in 2013 IEEE Grenoble Conference, 2013, pp. 1-6.

[123] V. Salehi, A. Mazloomzadeh, and O. Mohammed, "Development and implementation of a phasor measurement unit for real-time monitoring, control and protection of power systems," in 2011 IEEE Power and Energy Society General Meeting, 2011, pp. 1-7.

[124] "IEEE Standard for Synchrophasor Measurements for Power Systems." pp. 1-61, 2011. 
[125] "IEEE Standard for Synchrophasor Data Transfer for Power Systems." pp. 1-53, 2011.

[126] Z. Huang, T. Faris, K. Martin, J. Hauer, C. Bonebrake, and J. Shaw, "Laboratory performance evaluation report of SEL 421 phasor measurement unit," Pacific Northwest Natl. Lab., 2007.

[127] "Division 847, Time and Frequency Division, Boulder, CO. Available:," National Institute of Standards and technology (NIST), Division 847, Time and Frequency Division, Boulder, CO. [Online]. Available: http://tf.nist.gov/.].

[128] "IEEE SA - C37.118.2-2011 - IEEE Standard for Synchrophasor Data Transfer for Power Systems.” [Online]. Available: http://standards.ieee.org/findstds/standard/C37.118.2-2011.html. [Accessed: 19May-2014].

[129] "IEEE SA - C37.118.1-2011 - IEEE Standard for Synchrophasor Measurements for Power Systems." [Online]. Available: http://standards.ieee.org/findstds/standard/C37.118.1-2011.html. [Accessed: 19May-2014].

[130] "1547-2003 - IEEE Standard for Interconnecting Distributed Resources With Electric Power Systems," 2003.

[131] O. Samuelsson and N. Strath, "Islanding detection and connection requirements," in 2007 IEEE Power Engineering Society General Meeting, 2007, pp. 1-6.

[132] K. Maki, A. Kulmala, S. Repo, and P. Jarventausta, "Problems related to Islanding Protection of Distributed Generation in Distribution Network," in 2007 IEEE Lausanne Power Tech, 2007, pp. 467-472.

[133] M. A. Redfern, O. Usta, and G. Fielding, "Protection against loss of utility grid supply for a dispersed storage and generation unit," IEEE Trans. Power Deliv., vol. 8, no. 3, pp. 948-954, Jul. 1993.

[134] C. Diduch, "Recent developments in islanding detection for distributed power generation," in 2004 Large Engineering Systems Conference on Power Engineering (IEEE Cat. No.04EX819), pp. 124-128.

[135] D. Laverty, D. J. Morrow, and T. Littler, "Internet based loss-of-mains detection for distributed generation," in 2007 42nd International Universities Power Engineering Conference, 2007, pp. 464-469.

[136] M. E. Ropp, K. Aaker, J. Haigh, and N. Sabbah, "Using power line carrier communications to prevent islanding [of PV power systems]," in Conference 
Record of the Twenty-Eighth IEEE Photovoltaic Specialists Conference - 2000 (Cat. No.00CH37036), pp. 1675-1678.

[137] T. Funabashi, K. Koyanagi, and R. Yokoyama, "A review of islanding detection methods for distributed resources," in 2003 IEEE Bologna Power Tech Conference Proceedings, vol. 2, pp. 608-613.

[138] P. Mahat and B. Bak-Jensen, "Review of islanding detection methods for distributed generation," in 2008 Third International Conference on Electric Utility Deregulation and Restructuring and Power Technologies, 2008, pp. 2743-2748.

[139] W. Freitas, W. Xu, C. M. Affonso, and Z. Huang, "Comparative Analysis Between ROCOF and Vector Surge Relays for Distributed Generation Applications," IEEE Trans. Power Deliv., vol. 20, no. 2, pp. 1315-1324, Apr. 2005.

[140] F. De Mango, M. Liserre, and A. D. Aquila, "Overview of Anti-Islanding Algorithms for PV Systems. Part II: ActiveMethods," in 2006 12th International Power Electronics and Motion Control Conference, 2006, pp. 1884-1889.

[141] R. H. Lasseter, "Microgrids," in Power Engineering Society Winter Meeting, 2002. IEEE, 2002, vol. 1, pp. 305-308.

[142] R. H. Lasseter, "Extended CERTS microgrid," in Power and Energy Society General Meeting-Conversion and Delivery of Electrical Energy in the 21st Century, 2008 IEEE, 2008, pp. 1-5.

[143] W. M. Strang, C. J. Mozina, B. Beckwith, T. R. Beckwith, S. Chhak, E. C. Fennell, E. W. Kalkstein, K. C. Kozminski, A. C. Pierce, P. W. Powell, and others, "Generator synchronizing industry survey results," Power Deliv. IEEE Trans., vol. 11, no. 1, pp. 174-183, 1996.

[144] F. Blaabjerg, R. Teodorescu, M. Liserre, and A. V Timbus, "Overview of control and grid synchronization for distributed power generation systems," Ind. Electron. IEEE Trans., vol. 53, no. 5, pp. 1398-1409, 2006.

[145] C. Cho, S.-K. Kim, J.-H. Jeon, and S. Kim, "New ideas for a soft synchronizer applied to CHP cogeneration," Power Deliv. IEEE Trans., vol. 26, no. 1, pp. 1121, 2011.

[146] H. S. Timorabadi and F. P. Dawson, "A wide-range synchronization system for ac power systems," in Industrial Electronics, 2006 IEEE International Symposium on, 2006, vol. 3, pp. 1667-1672.

[147] A. J. Grono, "Synchronizing generators with HITL simulation," Comput. Appl. Power, IEEE, vol. 14, no. 4, pp. 43-46, 2001. 
[148] A. J. Grono and J. J. Wlodarski, "Setting and testing automatic generator synchronizers," Comput. Appl. Power, IEEE, vol. 12, no. 1, pp. 38-40, 1999. 
VITA

\section{ALI MAZLOOMZADEH}

1983

$1994-2000$

2000-2001

2001-2005

2006-2009

$2010-2014$

2013

2014
Born, Tehran, Iran

National Diploma, Mathematics and Physics National Organization for Development of Exceptional Talents, Karaj, Iran

Pre-University Certificate, Mathematics and Physics National Organization for Development of Exceptional Talents, Karaj, Iran

B.S., Electrical Engineering Islamic Azad University, Tehran, Iran

M.S., Electrical Engineering Amirkabir University of Technology, Tehran, Iran

Doctoral Candidate, Electrical Engineering Florida International University, Miami, Florida

Award, Best Student Paper

IEEE Smartgridcomm. International Conference, Canada

Award, Dissertation Year Fellowship

Florida International University, Miami, Florida

\section{SELECTED PUBLICATIONS AND PRESENTATIONS}

[1] Mazloomzadeh, Ali; Mohammed, Osama; Zonouz, Saman, "Trusted Sensing Base for Power Infrastructures", IEEE Transactions on Smart Grid, Special Issue on Cyber Physical Systems and Security for Smart Grid

[2] Barzegaran, M.; Mazloomzadeh, A.; Mohammed, O.A., "Fault Diagnosis of the Asynchronous Machines Through Magnetic Signature Analysis Using Finite-Element Method and Neural Networks," IEEE Transactions on Energy Conversion, vol.28, no.4, pp.1064,1071, Dec. 2013

[3] V. Salehi, A. Mohamed, A. Mazloomzadeh, and O. A. Mohammed, "Laboratory-Based Smart Power System, Part I: Design and System Development," IEEE Transactions on Smart Grid, vol. 3, pp. 1-11, 2012. 
[4] V. Salehi, A. Mohamed, A. Mazloomzadeh, and O. A. Mohammed, "Laboratory-Based Smart Power System, Part II: Control, Monitoring, and Protection," IEEE Transactions on Smart Grid, vol. 3, pp. 1-13, 2012.

[1] Ali Mazloomzadeh, Hazar Cintuglu, Osama Mohammed "Implementation and Integration of Phasor Measurement Units in Laboratory Based Smartgrid Testbed " International Conference on Engineering Education and Research (ICEER2014-McMaster), Ontario, CAN, 24-26 Aug. 2014.

[2] Ali Mazloomzadeh, Hazar Cintuglu, Osama Mohammed "Development and Evaluation of a Laboratory Based Phasor Measurement Devices". Innovative Smart grid Technologies, IEEE Conference, Washington DC, Feb 2015

[3] Barzegaran, M.R.; Mazloomzadeh, A.; Mohammed, O.A., "Identification of short-circuit location in induction motors using radiated electromagnetic field signatures," Electrical Machines and Systems (ICEMS), 2013 International Conference on , vol., no., pp.484,489, 26-29 Oct. 2013

[4] Mazloomzadeh, Ali; Mohammed, Osama; Zonouz, Saman, "TSB: Trusted sensing base for the power grid," Smart Grid Communications (SmartGridComm), 2013 IEEE International Conference on , vol., no., pp.803,808, 21-24 Oct. 2013

[5] Mazloomzadeh, A.; Farhadi M.; Mohammed, O.; , " Hardware Implementation of Hybrid AC-DC Power System Laboratory Involving Renewable Energy Sources", American Society for Engineering Education(ASEE) Annual Conference, Atlanta, USA, 18-26 June 2013

[6] Mazloomzadeh, A.; Cintuglu M. H.; Mohammed, O.; , " Islanding Detection Using Synchronized Measurement in Smart Microgrids" Innovative Smart Grid Technologies , Latin America(ISGTLA), 2013 IEEE PES, Brazil, 15-17 April 2013.

[7] Mazloomzadeh, A.; Salehi, V.; Mohammed, O.; , "Soft synchronization of dispersed generators to micro grids for smart grid applications," Innovative Smart Grid Technologies (ISGT), 2012 IEEE PES , vol., no., pp.1-7, 16-20 Jan. 2012

[8] Salehi, V.; Mazloomzadeh, A.; Mohammed, O.; , "Real-time analysis for developed laboratory-based smart micro grid," Power and Energy Society General Meeting, 2011 IEEE, vol., no., pp.1-8, 24-29 July 2011

[9] Salehi, V.; Mazloomzadeh, A.; Mohammed, O.; , "Development and implementation of a phasor measurement unit for real-time monitoring, control and protection of power systems," Power and Energy Society General Meeting, 2011 IEEE , vol., no., pp.1-7, 24-29 July 2011

[10] Saghaleini, M.; Mazloomzadeh, A.; , "Agent based control scheme for a smart power system including renewable energy sources," Environment and Electrical Engineering (EEEIC), 10th International Conference on , vol., no., pp.1-4, Roma, Italy,8-11 May 2011 